\title{
Neurovision : neuroimaging studies of illusory perception
}

Citation for published version (APA):

Weigelt, S. B. (2008). Neurovision : neuroimaging studies of illusory perception. [Doctoral Thesis, Maastricht University]. Datawyse / Universitaire Pers Maastricht. https://doi.org/10.26481/dis.20081209sw

Document status and date:

Published: 01/01/2008

DOI:

10.26481/dis.20081209sw

Document Version:

Publisher's PDF, also known as Version of record

\section{Please check the document version of this publication:}

- A submitted manuscript is the version of the article upon submission and before peer-review. There can be important differences between the submitted version and the official published version of record.

People interested in the research are advised to contact the author for the final version of the publication, or visit the DOI to the publisher's website.

- The final author version and the galley proof are versions of the publication after peer review.

- The final published version features the final layout of the paper including the volume, issue and page numbers.

Link to publication

\footnotetext{
General rights rights.

- You may freely distribute the URL identifying the publication in the public portal. please follow below link for the End User Agreement:

www.umlib.nl/taverne-license

Take down policy

If you believe that this document breaches copyright please contact us at:

repository@maastrichtuniversity.nl

providing details and we will investigate your claim.
}

Copyright and moral rights for the publications made accessible in the public portal are retained by the authors and/or other copyright owners and it is a condition of accessing publications that users recognise and abide by the legal requirements associated with these

- Users may download and print one copy of any publication from the public portal for the purpose of private study or research.

- You may not further distribute the material or use it for any profit-making activity or commercial gain

If the publication is distributed under the terms of Article $25 \mathrm{fa}$ of the Dutch Copyright Act, indicated by the "Taverne" license above, 
Neurovision

neuroimaging studies of illusory perception 


\section{Colophon}

Cover design by Sarah Weigelt

Layout design by Sarah Weigelt with great help by Ellen Jongen

Printed by Datawyse bv, Maastricht / Universitaire Pers Maastricht

ISBN 9789052787893

Copyright (C) Sarah Weigelt, Frankfurt am Main 2008 


\section{Neurovision}

\section{neuroimaging studies of illusory perception}

\section{Proefschrift}

ter verkriiging van de graad van doctor aan de Universiteit Maastricht, op gezag van de Rector Magnificus, Prof. mr. G.P.M.F. Mols,

volgens het besluit van het College van Decanen,

in het openbaar te verdedigen

op dinsdag 9 december 2008 om 10.00 uur

door

Sarah Brigitta Weigelt

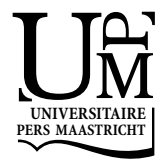




\section{Promotor}

Prof. dr. R. Goebel

\section{Copromotores}

Dr. L. Muckli

(University of Glasgow, UK)

Dr. A. Kohler

(Max Planck Institute for Brain Research, Germany)

\section{Beoordelingscommissie}

Prof. dr. C. Kemner (voorzitter)

Prof. dr. J. Kaiser

(University of Frankfurt, Germany)

Prof. dr. F. di Salle

Prof. dr. P. Stoerig

(University of Düsseldorf, Germany)

Prof. dr. P. de Weerd

The research reported in the present dissertation was conducted at the Department of Neurophysiology of the Max Planck Institute for Brain Research and at the Brain Imaging Center, Frankfurt am Main, Germany. Part of the research reported in Chapter 3 was conducted at the Department of Neurophysiology of the Max Planck Institute for Biological Cybernetics, Tübingen, Germany.

All research reported in the present dissertation was supported by the Max Planck Society. Research reported in Chapter 3, 4 and 5 was supported by the German Research Foundation (MU 2358/1-2) and the Federal Ministry of Education and Research (BMBF 01 GO 0508). Research reported in Chapter 3 was additionally supported by the German Research Foundation (TH 812/1-1). 


\section{Contents}

$\begin{array}{lll}\text { Chapter } 1 & \text { Introduction } & 7\end{array}$

Chapter 2 The method of functional magnetic resonance adaptation in visual neuroscience 21

Chapter 3 The cortical representation of objecst rotating in depth

Chapter 4 Separate cortical stages in amodal completion as revealed by functional magnetic resonance adaptation

Chapter 5 Patterns of $\mathrm{fMRI}$ activity representing perceived direction in apparent motion $\quad 85$

$\begin{array}{lll}\text { Chapter } 6 & \text { Discussion } & 97\end{array}$

$\begin{array}{ll}\text { Summary } & 179\end{array}$

$\begin{array}{ll}\text { Samenvatting } & 123\end{array}$

$\begin{array}{ll}\text { References } & 127\end{array}$

$\begin{array}{ll}\text { Abbreviations } & 147\end{array}$

Acknowledgements $\quad 149$

Curriculum Vitae 151 



\section{Introduction}


The question is not what you look at, but what you see.

- Henry David Thoreau

\section{‘Neurovision’ - neuroimaging investigations of illusory percep- tion}

The title 'Neurovision' refers to the two main themes of this dissertation. First, it reflects the fact that I investigated neuronal or more precisely cortical mechanisms underlying visual illusory perception. In more prosaic terms, one might say that although we look onto the world with our eyes, we see with our visual cortex. Second, the title reflects my optimistic view on neuroimaging techniques and my firm belief that although we are looking at a vascular signal, we are actually'seeing' neuronal activity.

In the following I will explain in more detail these two themes. Furthermore, the introductory chapter will give an overview on the visual phenomena that were under study as well as on the methodological approaches that were employed. Importantly, the introduction should serve two purposes: On the one hand, I tried to write the introduction in a way that enables laypersons to understand the ideas behind our research without having to read the original research papers that follow in Chapters 2-5. On the other hand, for the scientific audience I offer more background information - contentas well as method-wise - that is not provided by the original research papers.

\section{Neurovision part 1: looking with our eyes, seeing with our brain}

The first main theme of this dissertation is the focus on cortical processes in the course of visual perception. If you think about vision, first you will think about our eyes. We look at the world 'through our eyes'. But the expression 'through' already emphasizes the fact that our eyes are more or less a medium, a tool for visual perception. The actual processing that leads to conscious visual percepts occurs at the cortical level. This is not to say that there is no information processing already in the retina or subcortical level. Actually, a lot of pre-processing already takes place in these early steps of the visual hierarchy (Field and Chichilnisky, 2007; Wässle, 2004).

However, one of the most fascinating facts about cortical processing is that it is not purely forwarding information provided by the retinae, but it is an active and constructive process (Goebel et al., 1998; Gregory, 1966; Zeki et al., 1993). Cortical processing 'adds' something to visual perception that is not present in the actual world: color, for example. Objects are not colored the way we see them. They reflect light of a different wavelength and by complex mechanisms - that are still not fully understood - the conscious perception of colored objects emerges (Gegenfurtner, 2003). Or think about visual imagery. You are able to close your eyes and imagine someone's face, a ball rolling, or the view from outside your window. Thus, we seem to experience visual images without at the same time having retinal input.

Other interesting examples for the constructive nature of vision are visual illusions. Figure $1 \mathrm{~A}$ and $\mathrm{B}$ provides two examples. In Figure $1 \mathrm{~A}$ you will most certainly see a white triangle and three black dots. However, there is no white triangle actually present in the picture - the triangle is illusory. The illusory percept has been made up by your visual cortex. In Figure 1B you see a depiction of the so-called 'Necker cube'. Most certainly, your perception will change from time to time, that is, your percept shifts between the 
two possible interpretations where the corners are attributed to the front side or the back side. However, in reality the picture does not change. What changes is your cortical interpretation of the incoming signals. These fluctuations cause the change of your conscious percept.

There are numerous optical illusions like these that challenge vision scientists to unravel their underlying cortical mechanisms. By studying visual illusions (and related phenomena) we thus get to know more about the constructive nature of vision.

A

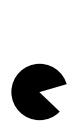

B

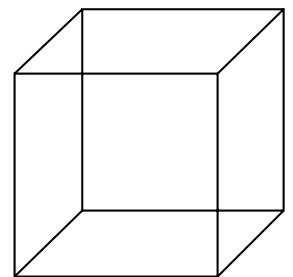

Figure 1. Visual illusions. A, depicts a typical 'Kanizsa triangle', an illusory white triangle on top of three black circles. B, depicts the 'Necker cube', a cube that changes appearance from time to time and thus presents a case of perceptual bistability.

\section{Neurovision part 2: looking at a vascular signal, seeing neuronal ac- tivity}

The second main theme is motivated from methodology. Based on the first theme that I want to unravel neuronal circuits that underlie visual perception, I have to find a method that allows me to study brain activity. The present dissertation employs functional magnetic resonance imaging (fMRI). FMRI is a non-invasive neuroimaging technique that detects changes in blood flow related to neuronal activity. Being thus an indirect measurement of neuronal activity, the term 'Neurovision' should emphasize the fact that I anyway believe that we are actually'seeing' neuronal activity. I think that fMRI has proven to be a potent method to measure brain activity. Still, further research is clearly needed to disentangle the exact correspondence of neuronal and vascular signaling cascades.

Furthermore, I want to stress the importance of advanced neuroimaging approaches that might offer a closer look on neuronal processing than the conventional designs. The two advanced neuroimaging approaches used in the present dissertation are $\mathrm{fMRI}$ adaptation and multi-voxel pattern classification. The first is a development in experimental design, while the second is a recent advancement in data analysis. Both approaches have in common that they allow extended conclusions about underlying neuronal processing that are not derivable from conventional designs or analysis techniques. I am perfectly aware of the fact that both methods have to be validated further to prove their claims. However, I have the 'vision' that although measuring a vascular signal we are still able to see neuronal activity.

Since I want to stress the point that the phenomena under study actually occur in our daily lives, I will take you to a visual scene that gives examples of all three phenomena. Now, imagine going to a typical German Christmas Market (Figure 2). Of course you might first go straight to the mulled wine booth, but then, holding the hot cup 
in your hand, you might stroll around the market and finally stand in front of a huge, beautifully decorated merry-go-round.

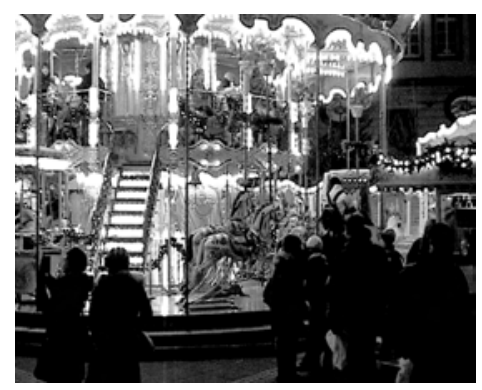

Figure 2. Merry-go-round scene.

\section{Apparent rotation}

While we observe the merry-go-round in motion, something fascinating happens in our brain. Every moment the whole scene changes, the rotating motion of the carousel and everything that's on it, casts continuously changing scenes onto our retina. If we observe the carousel's staircase and horses in more detail, we see that every moment in time, they appear different. Because of the rotation, parts become occluded, while other parts are revealed (Figure 3). Still, our perception is that of a smoothly moving object. We do not see multiple objects, but only one object that changes its appearance over time. Thus, we may conclude that our visual system is able to keep track of objects albeit them changing in shape or illumination. In other words, our visual system binds these different views of an object, thereby creating a coherent percept. Now what happens, if we do not see every single frame of the moving object? We might blink or look away for a very short time, and still, our percept is that of a smoothly moving object. It seems that our visual system somehow reconstructs the missing information, and here is where the phenomenon of apparent rotation comes into play.
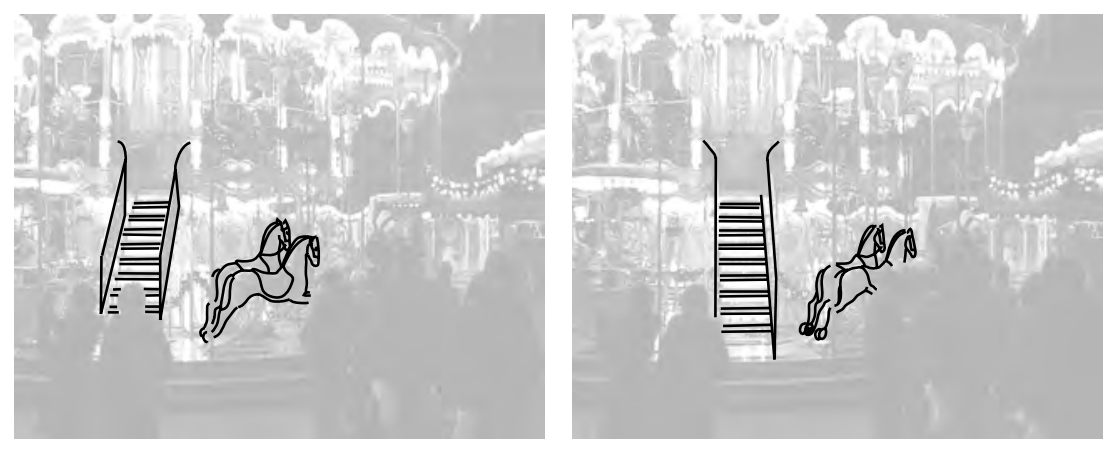

Figure 3. Merry-go-round scene demonstrating the phenomenon of apparent rotation. Two frames of a video depicting a rotating merry-go-round were taken to illustrate the effect of rotational motion on object shape. 
'Apparent rotation' refers to a visual illusion of rotation of three-dimensional objects (Farrell and Shepard, 1981; Shepard and Judd, 1976). The illusion was first described by Shepard and Judd (1976). They used perspective views of three-dimensional (3D) objects (Figure 4). By presenting two perspectives of the same 3D object in rapid succession, a visual illusion is elicited of one object rotating rigidly back and forth. That is, our visual system fills-in the missing intermediate perspectives, generating the illusion of a smoothly moving object despite the fact that only two perspectives were presented. Interestingly, the two perspectives can be separated by quite big angular differences, and still the illusion would persist. Shepard and Judd (1976) thoroughly investigated the relationship between the strength of the illusion and the angular difference between the two perspectives on the one hand and the duration of the stimulus presentation on the other hand. In more experimentalist's terms, the authors determined for each of several angular differences the shortest delay between stimulus onsets (stimulus onset asynchrony, SOA) for which the subject was still able to experience the illusion. Their main result was that the minimum SOA at which the subject still saw apparent rotation increased linearly with the angular difference between the two perspectives. This linear relationship had the same slope for in-depth as for in-plane rotations.
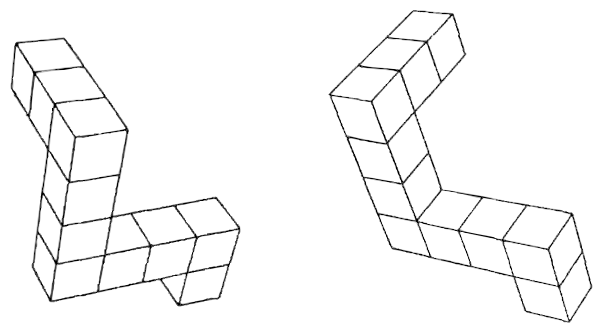

Figure 4.The phenomenon of apparent rotation. The figure depicts two perspective views of a three-dimensional tetris-like object as were used in the original study by Shepard and Judd (1976).

Interestingly, Shepard and Metzler (1971) had previously investigated mental rotation and found a similar linear relationship between the time subjects required to determine whether two perspectives refer to the same 3D object and the angular difference between the two perspectives. It is important to note that apparent rotation in comparison to mental rotation is of a much more perceptual nature. It does not involve an active, cognitive process, but is just experienced in an effortless way. Hence, SOAs for apparent rotation were found to be much smaller compared to SOAs for mental rotation (Shepard and Judd, 1976).

From a neuroscientific point of view, the question immediately arises, which intricate neuronal mechanism might underlie the illusion of apparent rotation. More precisely, we might ask where in the brain those illusory, intermediate rotation positions are being represented. This was the research question that we addressed in our study of apparent rotation (Chapter 3). However, we also want to stress the point that the investigation of a visual illusion is not only interesting by itself, it also teaches us a lot about our visual system in general. In the case of apparent rotation, what we actually 
learn is how two features of an object, namely its viewpoint and motion, are linked and that this linking has a major impact on object recognition.

In this respect, the phenomenon of apparent motion sheds some light on a vivid debate that started in the 70s, got fierce in the 90 s and just recently seems to be waning (Hayward, 2003). The debate - often referred to as 'the viewpoint debate' - in a nutshell is on whether object recognition is viewpoint invariant or viewpoint dependent. The general problem is that the same object looks different across changes in viewpoint, but still we are able to recognize objects without difficulty. How do we overcome this obstacle? Two opposing answers emerged from the research community that split experimental psychologists into two embittered parties. Either the problem is solved because objects are generally represented in an 'object-centered' (viewpoint invariant) fashion or objects are generally represented in a 'viewer-centered' way and we have to learn to recognize objects from different perspectives. Numerous psychophysical as well as neuroscientific investigations were devoted to address this vexing puzzle (Biedermann and Bar, 1999; Biederman and Gehardstein, 1993; Bülthoff and Edelman, 1992; Logothetis et al., 1994; Marr and Nishihara, 1978; Tarr and Bülthoff, 1995; Tarr and Pinker, 1989). However, experimental evidence seemed to support both positions equally well. Finally, the opponents came to the agreement that both models play a role in object recognition and that specific recognition tasks might favor one over the other (Jolicoeur, 1990) or that they act in a combined fashion (Foster and Gilson, 2002).

The whole debate concentrated on static image displays. However, viewpoint changes are either due to the movement of the object or of the observer. The impact of motion on the representation of objects - especially their viewpoint - was investigated by Kourtzi and Shiffrar (Kourtzi and Shiffrar, 1997; 1999; 2001). They tested whether subjects achieve view-invariant object recognition more readily with moving than with static objects. In their 'apparent-motion condition' they presented an apparent-rotation sequence comprising two perspectives of one object at the center of the screen and with an interstimulus interval (ISI) of $0 \mathrm{~s}$. In contrast, in the 'static condition' the two perspectives were displayed on the right and the left side of fixation respectively and the ISI was $450 \mathrm{~ms}$. This spatiotemporal discrepancy eliminated the perception of apparent rotation in the static condition. Following these prime sequences was an ISI of 500 ms. Then a pair of targets was presented and subjects had to press a button to indicate whether the two targets matched or not ('immediate priming paradigm', Sekuler and Palmer, 1992). Thus, the priming sequence shown before was completely irrelevant for the task. By varying the orientation (rotation) of the targets, Kourtzi and Shiffrar were able to reveal the effect of the (apparent) rotation onto the representation of intermediate rotation positions. Their main result was that target orientations falling in-between the two prime perspectives were primed across all rotation angles in the apparent-rotation condition, while in the control conditions these intermediate rotation positions were only primed for small angles (Kourtzi and Shiffrar, 1997). In other words, intermediate rotation positions are actually represented by the visual system. Thus, motion fosters view-invariant object recognition.

The studies of Kourtzi and Shiffrar were followed by a recent investigation of apparent rotation by McNamara and colleagues (2006). In contrast to Kourtzi and Shiffrar, McNamara and coworkers used an explicit measure of recognition rather than implicit priming. They presented configurations of dots, where the whole configuration would 
rotate in depth to create the apparent rotation illusion (McNamara et al., 2006). After the apparent rotation sequence, a test configuration was displayed and subjects had to indicate whether the configuration was 'old' or 'new' to them. The test configuration could be either (1) unfamiliar but within the range of rotation, (2) unfamiliar and beyond the range of rotation or (3) a novel configuration of dots. Response latency increased approximately linearly with angular distance for test configurations beyond the range of the apparent rotation. Most interestingly, latencies were not affected by angular distance within the range of rotation (McNamara et al., 2006). The authors conclude that the mechanism responsible for the perception of apparent rotation produces representations of the rotating object along its trajectory of motion (but not beyond). As Kourtzi and Shiffrar before (Kourtzi and Shiffrar, 1999), McNamara and colleagues (2006) hypothesize that an interaction between the 'what' and the 'where' path of the visual system (Ungerleider and Mishkin, 1982) might underlie the phenomenon of apparent rotation, constituting view-invariant object representations along the rotation path.

In our neuroscientific investigation of apparent rotation described in Chapter 3 we addressed exactly that question: Which brain regions are involved in the processing of apparent rotation? More precisely, which brain regions represent the unseen, intermediate rotation positions?

\section{Perceptual completion}

One of the most common visual phenomena is that of occlusion. If we look again at our carousel scene, we notice that almost every object in the scene is partly occluded: the carousel horse is partly occluded by the child riding it; the child's hand is partly hidden behind the horse's neck and so on (Figure 5). Nevertheless, we perceive those partly occluded objects automatically as whole and complete. For most of us, this observation seems too obvious to be important. However, for scientists this phenomenon is still a riddle. All those objects cast incomplete images on our retinae and our visual system somehow seems to complete the missing information by interpolating the occluded contours. But how? In the end, we perceive that there is no part missing, although we do not see all parts. This ordinary, but still puzzling phenomenon is called 'perceptual (visual) completion' (Tse, 1999).

By using the word 'perceiving' we - and other researchers before - want to highlight the sensory quality of perceptual completion. Even though there is no direct physical experience of the occluded part of the object, we 'see' this part in a sense that is different from just knowing or guessing that the object is complete. Evidence for the more perceptual than conceptual nature of perceptual completion comes from studies in the field of developmental psychology and ethology. Two-month old infants perceptually complete a moving bar behind an occluding bar, while four-month old infants do so even with static bars (Johnson, 2004). Infants under one month of age can perceive the continuation of a grating behind an occluding surface (Kawabata et al., 1999). From these studies we can conclude that perceptual completion is one of the fundamental mechanisms in human vision. 


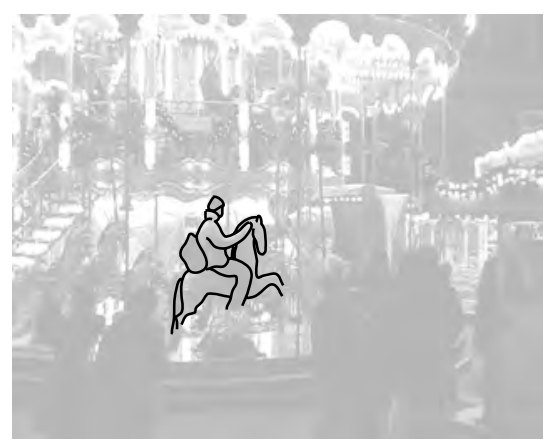

Figure 5. Merry-go-round scene demonstrating the phenomenon of perceptual completion. Many objects are occluded by other objects in this scene: For example the boy occludes part of the carousel horse, and still we perceive those objects as whole and complete.

But perceptual completion is not only evident in human vision. Animal studies have revealed that baboons (Deruelle et al., 2000), macaque monkeys (Fujita, 2001), mice (Kanizsa et al., 1993), and fish (Sovrano and Bisazza, 2008) are able to visually complete partly occluded objects. Interestingly though, pigeons seem not to have this visual capability (Fujita and Ushitani, 2005; Sekuler et al., 1996; Ushitani and Fujita, 2005). From these studies we might conclude that perceptual completion is a fundamental capability in most species, but not in all, and it still needs to be investigated which factors determine perceptual completion.

From an experimentalist's perspective, several interesting questions arise concerning perceptual completion: what are the differences between completed and real contours - does the visual system treat completed contours like real contours? What factors determine how we complete the hidden object? Do we actually make use of occluded contours, that is, does perceptual completion aid object recognition? Which brain processes are involved in perceptual completion? The first experimentalist who studied perceptual completion was Albert Michotte (Michotte, 1964; Wagemans et al., 2006). The Belgian experimental psychologist also coined the terms 'amodal completion' and 'modal completion'. Amodal completion refers to the perceptual completion of a hidden contour or object. In contrast, modal completion refers to illusory contours as demonstrated by the famous Kanizsa triangle (Kanizsa, 1976). The difference between amodal and modal completion is not easily defined. As a starting point, we might say that the major difference lies in the type or strength (this is a matter of discussion) of the completed percept. In the case of modal completion, we have a strong, vivid impression of a contour. As typical for visual illusions, we cannot directly tell whether the contour is really there or whether we are fooled by our visual system. In our classical example of a Kanizsa triangle in Figure 6A, we have to cover the inducing black spots to see that there really is no white triangle in-between. In the case of amodal completion, we have an impression of an existing, but hidden contour. If we look at the line drawing in Figure $6 \mathrm{~B}$, we tend to see a circle that is occluded by a square. We would be quite surprised, if it were revealed to us that the circle was actually a pacman-like shape merely abutting the square. We enter a philosophical debate, 
if we want to know, whether the percepts of an existing or a hidden contour are qualitatively or 'just' quantitatively different from each other. This is not in the scope of the thesis, but we will come back to the similarities and differences between amodal and modal completion in the discussion, when we evaluate neuroscientific findings. At this point, we want to emphasize that the research described in Chapter 4 is on the phenomenon of amodal completion.

Early gestalt psychologists assumed organizational principles such as 'simplicity of form' to account for amodal completion (Hochberg and McAlister, 1953). Later approaches focused either on contour completion (Kellman and Shipley, 1991; Wouterlood and Boselie, 1992) or surface completion (Nakayama et al., 1989). More recently it was shown that neither contour- nor surface-completion approaches can explain all completion phenomena, but that one has to take into account the volume of an object as well (Tse, 1999). Concerning the research question whether the visual system treats completed contours like real contours, the answer is:yes. In visual search experiments Rensink and Enns (1998) found that subjects take longer time to search for a pacman-like circle when this pacman-like circle abuts a square (and thus is amodally completed to a full circle behind a square and no longer perceived as a pacman-like circle) than when the pacman-like circle is just near a square, but not abutting it. Furthermore, Gold and colleagues (2000) showed that observers actually use perceptually completed contours to recognize objects. Later investigations focused more on the mechanism of amodal completion. In their seminal work on this topic, Sekuler and Palmer (1992) showed that the process of amodal completion starts with a representation of the (visible) physically presented object and ends with the perception of the completed object. Rauschenberger and Yantis (2001) demonstrate that the physical stimulus features are available to the visual system for a short time after stimulus presentation and are then abolished by the representation of the completed stimulus.

These investigations concerning the potential mechanism of amodal completion (Rauschenberger and Yantis, 2001; Sekuler and Palmer, 1992) suggest that the neuronal processes that underlie amodal completion involve separate stages. In our neuroscientific study of amodal completion described in Chapter 4 we addressed the question,

A

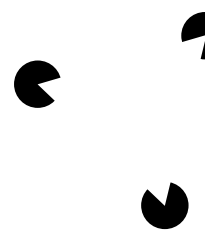

B

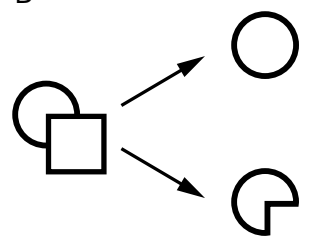

Figure 6. Modal and amodal completion. A, depicts a typical Kanizsa triangle (Kanizsa, 1976). Observers tend to see a white triangle covering three black spots at the tips. The illusory contour of the triangle is an example of modal completion. Interestingly, the covering of the black spots is an example of amodal completion - what is actually present are three pacman-like black shapes and not full circles. B, depicts on the left side a typical stimulus used in studies of amodal completion. Observers tend to report perceiving a square occluding a circle. Although the contour falling onto our retina resembles that of a pacman-like shape (lower right), we actually perceive a complete circle (upper right). 
which brain regions are involved in processing amodal completion. More precisely, we aim at localizing the different stages from the representation of the physical stimulus features to the representation of the amodally completed object.

\section{Apparent motion - perceived direction of motion}

A third phenomenon that is present in the merry-go-round scene can be observed by looking at the string of lights at the Christmas market booth. What we see are spots of light moving from left to right (or right to left) along the string of lights (Figure 7). However, this motion is illusory. In fact, the lights are 'just' switched on and off at specific intervals, thereby creating the impression of motion. No light bulb is really moving from left to right (or right to left). Although we might think that this illusory motion is seldom experienced, it actually underlies one of the most common visual experiences in our everyday life: cinematography. A 'motion picture' is created by presenting static
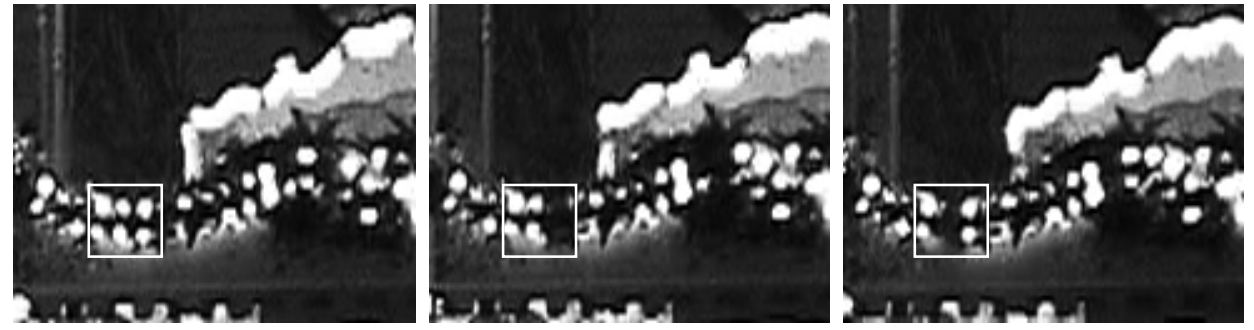

Figure 7. Close up of the merry-go-round scene demonstrating the phenomenon of apparent motion. Focusing on the string of light bulbs at the market booth in the right half of the picture, we can observe that the light bulbs are switched on and off one after the other. In reality, this creates the perception of moving light.

images in very rapid succession. The presentation rate is so high that we do not perceive the single, slightly changing frames but the smooth succession of changes.

This phenomenon is called 'apparent motion'. Scientifically, apparent motion can be defined as the illusory motion that we perceive when e.g. two stimuli are presented alternately at specific spatiotemporal intervals. We then see one stimulus moving up and down instead of two stimuli flashing at their respective locations (Figure 8A). Apparent motion has been studied extensively with psychophysical methods (Anstis et al., 1985; Kolers, 1964; Korte, 1915; Wertheimer, 1912). There it has been found that the perception of motion breaks down after prolonged stimulation (Anstis et al., 1985; Finley and von Grünau, 1987). Subjects then perceive flicker instead of motion. Phases of perceived apparent motion alternate with phases of perceived flicker - this characteristic of apparent motion is called bistability. Another interesting example of bistable apparent motion is the motion quartet (Hoeth, 1968; Neuhaus, 1930; Ramachandran and Anstis, 1983). If four stimuli are presented alternately at opposing corners of a square (and at specific spatiotemporal intervals) apparent motion is perceived either 
A
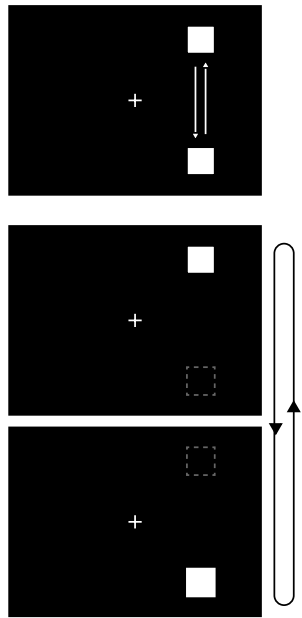

B
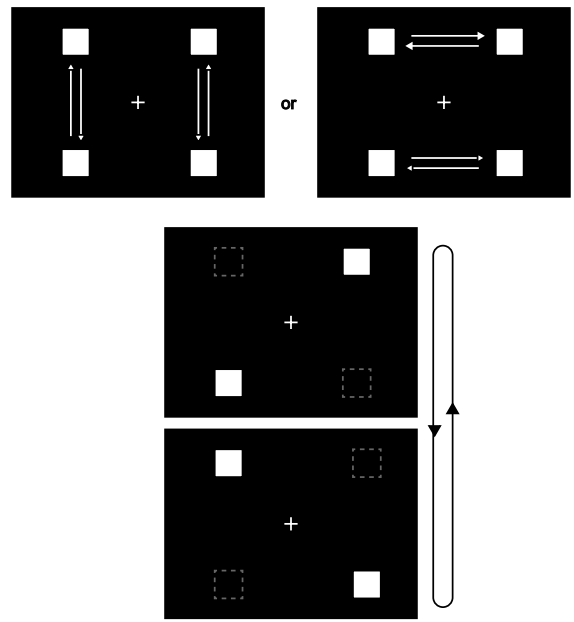

Figure 8. Apparent motion and the bistable apparent motion quartet. A, depicts a typical apparent motion stimulus. Two white squares are flashed in alternation at two different positions in the right visual field, thus producing vertical apparent motion. B, depicts the apparent motion quartet stimulus. Two opposing square pairs are flashed in alternation at the corners of an imaginary quartet, thus producing either vertical or horizontal apparent motion. The percept switches between the two motion directions (bistability).

in the vertical or in the horizontal direction (Figure 8B). Again, phases of perceived vertical apparent motion take turn with phases of perceived horizontal motion (and also flicker is sometimes seen). Studies investigating apparent motion have suggested that neuronal adaptation might underlie the bistable nature (Anstis et al., 1985; Clatworthy and Frisby, 1973; Finlay and von Grünau, 1987).

Neurophysiological (Mikami, 1991; Mikami et al., 1986a, b; Newsome et al., 1986) as well as neuroimaging (Goebel et al., 1998; Muckli et al., 2002;2005;Zeki et al., 1993) studies were conducted to unravel the neuronal mechanisms of apparent motion. Muckli et al. (2002) found that the human motion complex (hMT+) - a brain region dedicated to visual motion processing - plays a key role also in the processing of apparent motion. In their fMRI study, subjects were presented an apparent motion stimulus similar to the one displayed in Figure 7A and were instructed to report their percept (apparent motion or flicker). Activity in hMT+ was higher when subjects perceived apparent motion in contrast to flicker perception (Muckli et al., 2002). Sterzer et al. (2003) confirmed that area hMT+ is involved in the processing of apparent motion using the bistable motion quartet. Having established the central role of hMT+ in the perception of apparent motion, following neuroimaging studies focused on the role of early visual cortex (Larsen et al., 2006; Liu et al., 2004; Muckli et al., 2005; Sterzer et al., 2006). Muckli et al. (2005) and Larsen et al. (2006) found evidence for perceptual filling-in of the apparent motion trace in primary visual cortex (V1). They suggested that this filling-in was mediated by feedback activity from hMT+ to V1 (Muckli et al., 2005). Using effective connectivity methods, Sterzer and colleagues (2006) were able to confirm this hypothesis. 
Thus, the current working model of apparent motion involves area hMT+ and feedback connections to V1. What is still unclear is which brain regions may account for the bistable nature of apparent motion. Muckli and colleagues (2002; 2005) hypothesize that the different percepts are sustained by different neuronal populations in hMT+. The oscillations between motion and flicker perception or horizontal apparent motion and vertical apparent motion might be due to competing neuronal populations. Sterzer et al. $(2006 ; 2007)$ on the contrary, believe that the prefrontal cortex determines perceptual switches and states.

In our neuroscientific investigation of apparent motion described in Chapter 5 we addressed exactly that question: Which brain regions are involved in the perceived direction of motion in a motion quartet experiment? More precisely, can we extract the perceived direction of motion from activity in hMT+ and/or prefrontal cortex?

\section{FMRI adaptation}

In 2003 Paul Lauterbur and Peter Mansfield received The Nobel Prize in Physiology or Medicine "for their discoveries concerning 'magnetic resonance imaging'" (www. nobelprize.org). In a nutshell, magnetic resonance imaging (MRI) is an exact and noninvasive method based on the phenomenon of nuclear magnetic resonance that provides anatomical images of internal organs. The aforementioned prizes were not the only ones granted for the development of MRI - but the discoveries of Paul Lauterbur and Peter Mansfield were the ones that boosted the technique from the imaging of structure to the imaging of functions.

Functional magnetic resonance imaging (fMRI) using blood-oxygenation-leveldependent (BOLD)-contrasts started in the 90s (Ogawa et al., 1990), and this was the time when scientists studying perception and cognition became interested in using this technique. The three great advantages of $\mathrm{FMRI}$ in contrast to other neuroimaging techniques are (1) its non-invasive nature; it neither uses ionizing radiation nor radioactive tracer isotopes, and is thus - to all present knowledge - harmless, (2) its high spatial resolution, and (3) the fact that data from all parts of the brain can be obtained simultaneously. Hence, $\mathrm{fMRI}$ can be applied repeatedly and continuously and is suited for the investigation of nearly all brain functions. The application of $\mathrm{FMRI}$ in neuroscientific research has been growing enormously and technical advancements (higher field strength, faster gradient systems) have aided the evolution of fMRI even more. But, one should not forget that there is an existing limiting factor that is set by biology itself: $\mathrm{FMRI}$ is an indirect measurement of brain activity - the BOLD-signal is a vascular signal at the end of a long cascade initiated by active neurons, and thus the signal is temporally delayed and spatially blurred. Therefore, clever methodological advancements both of experimental designs as well as of data analyses are needed to circumvent these limitations. FMRI adaptation is such a methodological advancement in experimental design, while pattern classification is a recent development in dataanalysis strategies.

FMRI adaptation describes the fact that the fMRI signal attenuates due to prolonged or repeated stimulation in the particular brain region representing the stimulus (Grill-Spector et al., 2006; Krekelberg et al., 2006). FMRI-adaptation effects indicate 
neuronal adaptation effects, although the exact mechanisms of both phenomena and their relationship are still not fully understood. The great potential of this method is that it enables the assessment of functional profiles of brain regions at the subvoxel level. FMRI adaptation has been used to demonstrate orientation-selective responses in V1 (Fang et al., 2005; Larsson et al., 2006), which has proven to be difficult using conventional $\mathrm{fMRI}$ designs. The basic idea is that a neuronal subpopulation is adapted by the presentation of a specific stimulus (a vertical grating) and then a test stimulus is presented to assess the adaptation. The test stimulus is either a repetition of the adaptor stimulus (again a vertical grating) or a different stimulus (a horizontal grating). If the neuronal subpopulation does not display orientation-selective responses, the signal to the presentation of the different stimulus should be attenuated (as to the repetition of the adaptor stimulus). If the neuronal subpopulation does exhibit orientation selectivity, a rebound from adaptation, e.g. a higher signal should be observable. Based on this idea, the method of $\mathrm{fMRI}$ adaptation has been successfully applied in almost all domains of visual neuroscience (Grill-Spector et al., 2006; Krekelberg et al., 2006).

We evaluate the method of fMRI adaptation in detail in Chapter 2, the present paragraph aims merely at explaining why this method was employed in our studies of apparent rotation (Chapter 3) and amodal completion (Chapter 4). Therefore, we have to come back to the research questions on apparent rotation and amodal completion expounded before. Both studies have in common that we aim at localizing the neuronal representations of illusory percepts. In the one case, we search for the representation of illusory rotation positions that are elicited via apparent rotation, while in the other case we look for the representation of an amodally completed shape. The trick using fMRI adaptation in the study of visual illusions works as follows: First, the stimulus eliciting the visual illusion is presented. Second, a test stimulus is presented. This test stimulus can either reflect the illusory percept or depict the actual physical stimulus. The rationale is that brain regions representing the illusory percept should show an adapted signal to the presentation of the 'illusory' test stimulus, while brain regions representing the physical stimulus should show an adapted signal to the presentation of the 'physical' stimulus. In both cases, it is of utmost importance to test the adaptation against test (control) stimuli that would not elicit adaptation effects (e.g. because they had never been presented before). Thus, by employing fMRI adaptation in the study of visual illusions, we are able to disentangle brain activity related to mere physical stimulus processing from brain activity indicating the (illusory) percept. Apart from one other study on illusory contours (Montaser-Kouhsari et al., 2007), to our knowledge we are the first to use fMRI adaptation in the study of illusions.

\section{Multi-voxel pattern analysis}

As stated above, the method of multi-voxel pattern analysis (MVPA; Norman et al., 2006) is a recent advancement in analysis techniques of imaging data. The basic idea behind it is that the functional architecture of the brain rather consists of distributed and overlapping representations than of spatially segregated clusters (Haxby et al., 2001). Haxby and coworkers (2001) demonstrated that the representations of faces and objects in ventral visual cortex - normally thought of as a textbook example for 
a modular organization (Op de Beek et al., 2008) - are widely distributed and overlapping. These 'patterns of activity' cannot be detected by classical fMRI data analysis using univariate statistics; multivariate statistics implemented in MVPA have to be used. MVPA is an umbrella term that denotes mathematical algorithms which extract patterns of activity from fMRI data. Since fMRI data is high-dimensional, patterns of activity can be detected both in space and time and space-time-coordinates. One of the great advantages of the method is that it allows extracting the pattern of response across multiple voxels, even if individual voxels might not show significant differences between the conditions of interest. Kamitani and Tong (2005) and Haynes and Rees (2005a) were thus able to decode the perceived orientation of a stimulus out of activity in $\mathrm{V} 1$, even though every single voxel of $\mathrm{V} 1$ only demonstrated a slight trend to be more responsive to one of the directions over the others.

MVPA has been employed to decode the attended orientation of a stimulus (Kamitani and Tong, 2005), the orientation of a stimulus that was effectively masked (Haynes and Rees, 2005a), the actual percept in binocular rivalry (Haynes and Rees, 2005a), and the seen and attended direction of motion (Kamitani and Tong, 2006) from activity in visual areas. Recently, Serences and Boynton (2007) were able to extract the attended direction of motion even from regions of visual cortex that were not directly stimulated thus confirming the idea that feature-based attention spreads across the cortex. The method has been also expanded to investigate more cognitive aspects such as intentions (Haynes et al., 2007). The possibility to extract perceptual or cognitive states out of brain data has made MVPA popular in a very short time. The method is often referred to as a 'brain reading' (Cox and Savoy, 2003; Haynes and Rees, 2006) or 'mind reading' technique (Norman et al., 2006). Given the overall popularity of neuroimaging methods in the media, MVPA has extended this general interest even more. It is important to note that beyond the fascinating and intimidating potential of actually reading somebody's internal states, MVPA is primarily employed to decipher the neuronal code.

The first MVPA were based on mere correlation analysis (Haxby et al., 2001). Following were linear discriminant analyses (Haynes and Rees, 2005a, b) and linear support vector machines (Kamitani and Tong, 2005;2006). These methods were optimally suited to decode patterns of activity from pre-defined brain regions (e.g. retinotopic visual areas). A further advancement of the MVPA was made by Kriegeskorte and coworkers (2006). They introduced a 'searchlight approach' meaning that the MVPA searches among the whole brain to detect patterns of activity. Thus, one does not need to have an apriori hypothesis where to look for the patterns.

In our neuroscientific investigation on apparent motion that is described in Chapter 5 we employ MVPA to extract the perceived direction of motion from brain activity. Following up the introductory paragraph on apparent motion, we test the hypothesis whether the perceived direction of motion can be decoded from activity in visual and prefrontal cortex. 


\section{2 \\ The method of functional magnetic resonance adaptation in visual neuroscience}

Functional magnetic resonance imaging (fMRI) is a powerful non-invasive tool to investigate neuronal processing. In the last ten years a new methodological approach in the field of fMRI has been developed: $\mathrm{fMRI}$ adaptation. It has been found that the repetition of a stimulus leads to a decrease of the fMRI signal in the brain region that processes this stimulus. The phenomenon has been related to neuronal adaptation effects found in single-cell recordings. Since the first experiments that observed fMRI-adaptation effects, the method has been applied extensively to study various visual phenomena, such as the perception of motion, shape, objects, and orientation. The great advantage of $\mathrm{fMRI}$ adaptation is that it allows assessing the functional response profile of a brain region at a subvoxel level. The purpose of the current review is to evaluate the different experimental approaches used to elicit fMRI-adaptation effects. We discuss papers published in the domain of visual neuroscience that made use of $\mathrm{fMRI}$-adaptation paradigms. In doing so, we focus on methodological considerations concerning experimental design, stimulus presentation and influencing factors such as awareness and attention. In the course of this review, we show that different fMRI-adaptation designs capture heterogeneous neuronal adaptation effects. As the picture of the mechanisms underlying neuronal adaptation changes from simple synaptic fatigue to complex network interactions, the concept of fMRI adaptation has to be redefined.

Weigelt, S., Muckli, L., and Kohler, A. (2008). The method of functional magnetic resonance adaptation in visual neuroscience. Reviews in the Neurosciences, in press. 


\section{Introduction}

From the start of functional magnetic resonance imaging, $\mathrm{fMRI}$ signal attenuation due to prolonged stimulation has been observed (Figure 1A) (Bandettini et al., 1997; Frahm et al. 1992; Tootell et al., 1998a). In the following years, researchers from the field of perceptual memory and priming (Buckner et al. 1995; 1998; Stern et al., 1996) were the first to notice fMRI signal decreases when comparing the repetition of a stimulus to the presentation of two different stimuli (Figure 1B). In their seminal work on visual object priming, Buckner et al. (1998) demonstrated decreased fMRI signals in extrastriate visual cortex following the brief, repeated presentation of visual objects. Furthermore, the repeated presentation of visual objects also led to behavioral priming effects ever since then, it has been argued that decreased $\mathrm{fMRI}$ signals due to repetition might be the neuronal correlate of behavioral priming effects. In direct succession, Grill-Spector and colleagues (1999) studied repetition-related fMRI-signal decreases to infer the selectivity of a specific visual brain region for a stimulus feature. This can be accomplished by first adapting a specific neuronal population (e.g., orientation-selective cells in primary visual cortex [V1]) with a repetitive presentation of a specific stimulus (e.g., a vertical grating). In a second step, the stimulus is varied along a predefined dimension (e.g., orientation) and the fMRI response to this change of the visual region under study is assessed. It is argued that, if the underlying neuronal population is indifferent to the change of the stimulus (does not exhibit orientation selectivity), the fMRI signal will be reduced similarly to the repetitive stimulation with the identical stimulus. On the contrary, if the neuronal population is sensitive to the change (does exhibit orientation selectivity), a rebound from adaptation will be observed - the signal will return to the non-adapted level. Based on this rationale, the technique of $\mathrm{fMRI}$ adaptation has become a powerful tool to characterize the functional profile of brain regions. Most importantly, fMRI adaptation is thus able to reveal response properties of neuronal populations at subvoxel resolutions.

The popularity of the method of fMRI adaptation is reflected in a large number of studies that made use of $\mathrm{FMRI}$-adaptation effects and several recent reviews summarizing the field (Grill-Spector et al., 2006; Krekelberg et al., 2006). The phenomenon is most commonly referred to as 'fMRI adaptation' (Boynton and Finney, 2003; Engel, 2005; Fang et al, 2005; Huk et al., 2001; Kourtzi and Kanwisher, 2000; Larsson et al, 2006; Weigelt et al., 2007a, b) or 'repetition suppression' (Henson et al., 2002; 2004; James and Gauthier, 2006; Sayres and Grill-Spector, 2006), where both terms strongly relate to neuronal adaptation effects that have been observed in electrophysiological recordings (Li et al., 1993; Miller et al., 1993; Ringo, 1996; Sobotka and Ringo, 1994). Until today however, the underlying neuronal mechanisms of fMRI adaptation are still not fully understood. More descriptive terms have also been used such as 'repetition attenuation' (Turk-Browne et al., 2006; 2007; Yi and Chun, 2005; Yi et al., 2006; Xu et al., 2007) and 'repetition-related response reduction' (Zago et al., 2005). We will adhere to 'fMRI adaptation' since it captures the correspondence of $\mathrm{FMRI}$ and neuronal adaptation effects (Kohn and Movshon, 2003; 2004; McMahon and Olson, 2007; Müller et al., 1999; Priebe and Lisberger, 2002; Priebe et al., 2002). More precisely, we will use the term 'fMRI adaptation' to name the technique, and the term 'fMRI-adaptation effect' to refer to the observable fMRI-signal decreases. 


\section{A FMRI adaptation after prolonged stimulation}
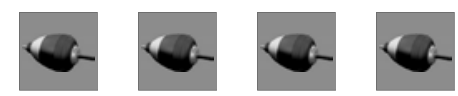

continuous presentation

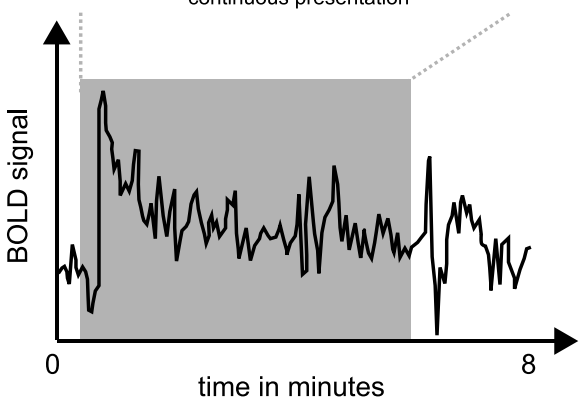

\section{B FMRI adaptation after repeated stimulation}

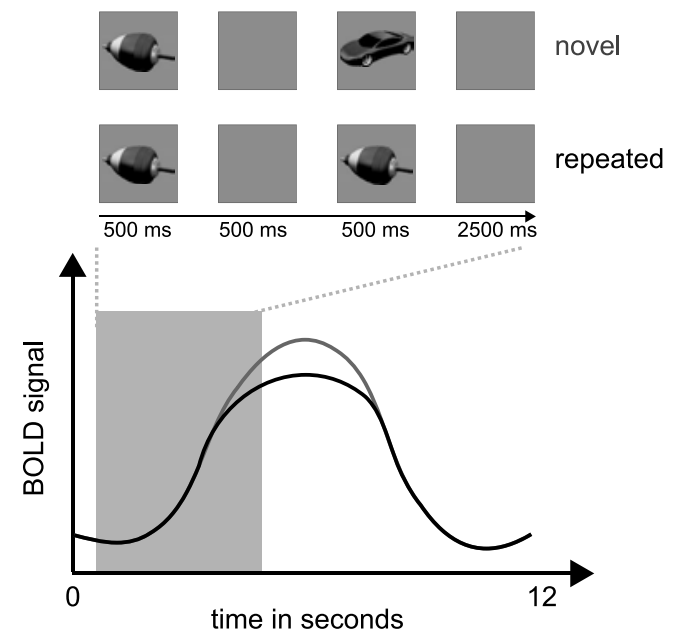

Figure 1. FMRI adaptation. A, schematic illustration of the adapting fMRI signal due to prolonged stimulation (over minutes). (Figure is adapted from Human Brain Mapping, Vol. 5, No. 2, 1997, 93-109; Copyright, 1997 by Wiley-Liss, Inc., reprinted with the permission of Wiley-Liss, Inc., a subsidiary of John Wiley \& Sons, Inc.). B, schematic illustration of a short-lag adaptation effect based on stimulus repetition that can be observed at the fMRI signal time course. (Object images courtesy of Michael J. Tarr, Brown University, http://www.tarrlab.org/) 
Interestingly, as diverse as the naming of the phenomenon are the experimental approaches that aim at eliciting fMRI-adaptation effects. Thus, a first goal of this review is to sort the different experimental designs in use and to compare their efficiency of evoking fMRI-adaptation effects. In addition, we address in detail the most important design factors such as repetition lag, adaptation duration, number of repetitions, visibility, and attention. Based on these reflections, we argue that different experimental designs most likely target different neuronal adaptation processes. We further elucidate this finding by referring to electrophysiological investigations that studied neuronal adaptation.

\section{Experimental design}

Starting with those studies that explicitly made use of fMRI adaptation as a specific marker of neuronal processing and inspecting the underlying experimental designs we found that almost all study designs can be assigned to one of six different types (Figure 2). In the following we will describe those different design types on the basis of original experiments. We will also report which other studies employed the respective design and comment on why a specific design type might be appropriate for the study of specific features.

\section{Block design with different numbers of repetitions}

One of the classical studies in the field of fMRI adaptation is the investigation by GrillSpector and colleagues (1999). Their use of an fMRI-adaptation approach was motivated by the need to circumvent the spatial limitations of conventional fMRI methods in order to resolve the response properties of neuronal populations at a subvoxel level. Using conventional fMRI methods it was not possible to disentangle neuronal populations that, e.g., would code for different object views within one imaging voxel. GrillSpector et al. (1999) employed an experimental design that we will call 'block design with different numbers of repetitions' (Figure 2A). The primary idea behind this design is that the amount of signal decrease relative to a non-adapted reference activation should vary as a function of number of repetitions. Grill-Spector and co-workers (1999) repeated either the same object 32 times in a block, 16 times intermixed with one other object, 8 times intermixed with three other objects, 4 times intermixed with seven other objects, or they presented 32 different objects. They found clear fMRI-adaptation effects when objects were repeated (32 times or 16 times) in comparison to the blocks containing 32 different images in the ventral visual pathway (Grill-Spector et al., 1999).

Other studies employed similar experimental designs (Konen and Kastner, 2008; McKyton and Zohary, 2007; Yi et al., 2006). Block designs with different numbers of repetitions were also applied in a study investigating $\mathrm{fMRI}$-adaptation effects across species (Sawamura et al., 2005) and - by the same group - in a neurophysiological single-cell study (Sawamura et al., 2006). Sawamura et al. (2005) revealed highly similar fMRI-adaptation effects to repeated objects in macaques and human subjects. Secondly, testing the design in their single-cell study led to robust neuronal adaptation to 
A Block design with different numbers of repetitions

fully adapted (8 out of 8)

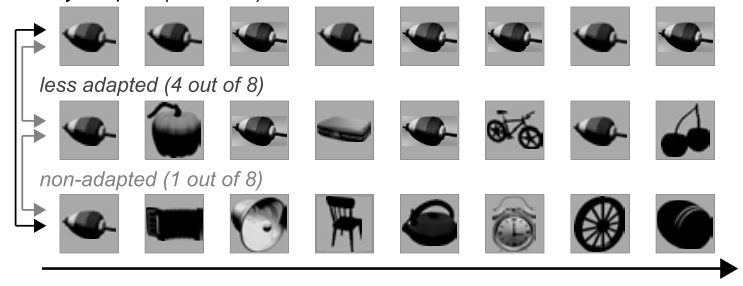

B Block design with image variation adaptation block

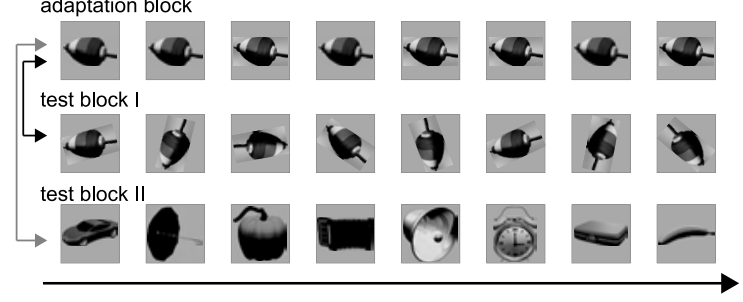

C Event-related design with pre-adaptation block pre-adaptation block

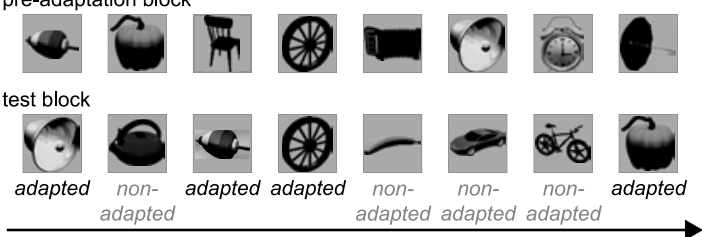

D Classic event-related design

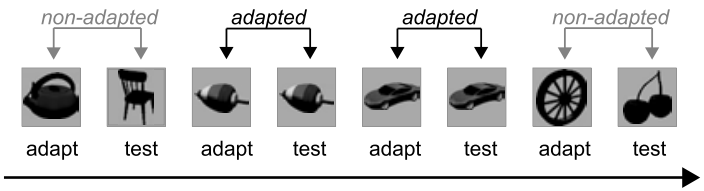

E Event-related design with pre-adaptation phase

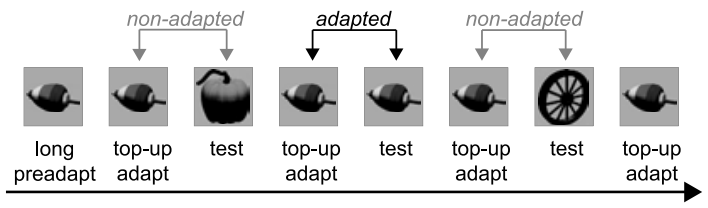

F Event-related design with intermixed presentation

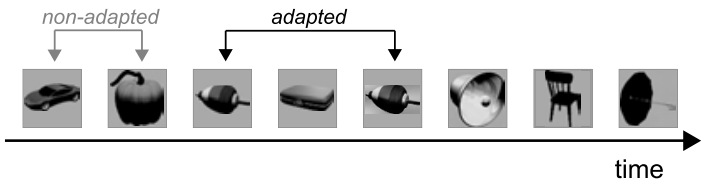

Figure 1. FMRI-adaptation designs. A-F, schematic illustrations of the different experimental designs that are used to elicit fMRI-adaptation effects. Stimulus presentation is shown against time and adaptor and test images/blocks are indicated. (Object images courtesy of Michael J. Tarr, Brown University, http://www.tarrlab.org/) 
repeated objects in the IT cortex of the macaque (Sawamura et al., 2006) demonstrating a good correspondence between $\mathrm{fMRI}$ and neuronal adaptation.

\section{Block design with image variation}

In the same study reviewed before, Grill-Spector et al. (1999) also applied what we will call a 'block design with image variation' (Figure 2B). In such a design, different blocks are contrasted, where all blocks involve the repeated presentation of a stimulus, but with different variations of stimulus dimensions such as size, position, viewpoint, and illumination. For example, Grill-Spector contrasted blocks in which a face was repeated with fixed illumination with blocks in which illumination of the same face (identity preserved) was changed from instance to instance (Grill-Spector et al., 1999). Thereby, the authors were able to show that subparts of the lateral occipital complex (LOC) show some invariance to size and position changes, but less so to illumination and viewpoint changes (for an extensive review of these findings see Grill-Spector et al., 2001). Other investigators employed a block design with image variation to study viewpoint effects on object representations (James et al., 2002), direction-selective adaptation (Nishida et al., 2003), size and viewpoint effects on face representations (Andrews and Ewbank, 2004), place representations (Ewbank et al., 2005), and response priming (Dobbins et al., 2004).

\section{Event-related design with pre-adaptation block}

Another classical study was conducted by Buckner and colleagues (1998). They employed what we will call an 'event-related design with pre-adaptation block' (Figure 2C). First, they presented a series of different object stimuli. They then showed the same objects again, intermixed with novel objects and fixation trials. Buckner et al. found fMRI-adaptation effects for repeated objects in comparison to novel objects in extrastriate areas, left dorsal prefrontal cortex, and anterior cingulate (Buckner et al. 1998). Furthermore, they also observed behavioral-priming effects as measured by decreased reaction times for repeated objects in comparison to novel objects (Buckner et al. 1998).

Because the repetition lag between the first presentation of the object and the repeated presentation typically is on the order of many seconds up to minutes and even days (van Turennout et al.2000;2003), this design is thought to reveal rather longlag adaptation effects. Those repetition effects are thought to be of cognitive nature, revealing processes of semantic memory more than stimulus-driven adaptation. Following the work by Buckner and colleagues (1998) the event-related design with preadaptation block has been used to investigate perception of faces (Eger et al., 2005), places (Epstein et al, 2005), and objects (Bunzeck et al., 2006; Ganel et al., 2006; James et al., 1999; 2000; 2006; Koutstaal et al., 2001; Simons et al., 2003; van Turennout et al., 2000; 2003, Vuillemier et al., 2002; Wig et al., 2005).

\section{Classic event-related design}

Most studies in the fMRI-adaptation field use what we call a 'classic event-related design' (Figure 2D). Every event consists of a rapid presentation of two consecutive stimuli that are only separated by a brief interval or none. The adaptor image is always immediately tested. Thus, the classic event-related design is thought to be the only design 
that identifies very short-lag adaptation effects. A paradigmatic study that made use of a classic event-related design was conducted by Kourtzi and Kanwisher (2000). They tested whether line drawings and grayscale photographs of objects are represented by the same neuronal subpopulation in LOC or by different populations (Kourtzi and Kanwisher, 2000). During scanning subjects were presented two images that depicted either the same object or different objects and that could be either of the same format (line drawing or photograph) or in different formats. Kourtzi and Kanwisher (2000) found $\mathrm{FMRI}$-adaptation effects in the LOC to the repetition of identical objects independent of their format and concluded that the LOC represents perceived shapes. Extending their own findings in further studies on object recognition always employing classic event-related adaptation designs (Kourtzi and Kanwisher, 2001, Kourtzi et al., 2003a, b, 2005), Kourtzi and colleagues strongly fostered the short-term adaptation technique. Many researchers followed (Ashida et al., 2007; Boynton et al, 2003; Eddy et al., 2007; Eger et al, 2004a, b; 2005, Epstein et al., 2003; 2005, Fang et al., 2007; Jiang et al., 2007; Krekelberg et al., 2005; Murray and Wojciulik, 2004; Murray, Olman et al., 2006; Rauschenberger et al., 2006; Sawamura et al., 2006; Valyear et al., 2006; Weigelt et al., 2007a, b; Welchman et al., 2005; Xu et al.,2007) The classic event-related design has also been tested in a recent electrophysiological single-cell study (Sawamura et al., 2006). There it was found that the design is able to elicit neuronal adaptation in neurons of IT cortex to repeated objects (Sawamura et al., 2006). Thus, we may conclude that the rapid event-related design reliably detects fMRI-adaptation effects in extrastriate cortex.

It has been debated to which degree fMRI-adaptation effects measured with eventrelated designs can be explained either by neuronal adaptation effects or non-linear neurovascular coupling (Boynton and Finney, 2003). As with every rapid event-related design the challenge is in determining the $\mathrm{fMRI}$ signal corresponding to a single event (trial) out of the highly convoluted signal time course. First estimations have suggested that fMRI signals add up almost linearly (Boynton et al., 1996; Dale and Buckner, 1997). However, a study by Huettel and McCarthy (2000) revealed that the response to a second stimulus following a first stimulus is significantly smaller than would be expected based on the assumption of linearity. Subjects were presented identical high-contrast checkerboard stimuli that were separated by inter stimulus intervals of 500,1500,3500 or 5500 ms while scanning. Huettel and McCarthy (2000) assessed the fMRI signal along the calcarine sulcus by subtracting the response to the pair of stimuli from the response to a single stimulus. They found that the response to the second stimulus decreased in amplitude and increased in latency with shorter repetition delays. Huettel and McCarthy (2000) attributed the observed non-linearity to a 'refractory period' in the $\mathrm{fMRI}$ hemodynamic response.

Direct empirical evidence that part of this non-linearity is indeed based on neuronal adaptation effects came from a study by Boynton and Finney (2003). They investigated orientation-specific adaptation since it is known that early visual areas contain orientation-specific neuronal subpopulations. By presenting either two gratings of the same orientation or two differently oriented gratings in rapid succession, Boynton and Finney (2003) found fMRI adaptation to repeated orientations in visual areas V3, and V4 (but not in V1/V2, see the following section on event-related designs with pre-adaptation phase for a possible explanation). At the same time, they tested contrast adapta- 
tion behaviorally as an indirect measure of neuronal adaptation. Further evidence that part of the non-linearities caused by rapid event-related designs are based on neuronal adaptation effects came from a study by Heckman et al. (2007). They modeled the effect of a fast design as a scaling of stimulus strength, which has been shown to be a typical consequence of neuronal adaptation. In addition, they could almost abolish non-linearities by using stimuli that would produce minimal adaptation effects.

We conclude that fast event-related designs are capable of detecting adaptation effects. Part of the non-linearity observed with rapid stimulation sequences can be attributed to adaptation, given the stimulation is well controlled (e.g., balancing trial history). Still, it is important to note that the design was unable to reveal orientationdependent adaptation effects in V1 and V2 and that such null effects pose a challenge to the interpretation of fMRI-adaptation effects.

\section{Event-related design with pre-adaptation phase}

An event-related design with pre-adaptation phase starts with a prolonged presentation of the adaptor stimulus over several seconds: $20 \mathrm{~s}$ (Fang et al., 2005), $25 \mathrm{~s}$ (Fang et al., 2007), $40 \mathrm{~s}$ (Liu et al., 2007), $60 \mathrm{~s}$ (Engel and Furmanski, 2001; Engel, 2005), $100 \mathrm{~s}$ (Larsson et al, 2006; Montaser-Kouhsari et al.2007), or even $150 \mathrm{~s}$ (Jurcoane et al., 2007). Following this pre-adaptation phase, is a classic event-related design in which the adaptor stimulus is now a so-called 'topping-up'-stimulus - the same stimulus as of the pre-adaptation phase shown again - followed by the test stimulus (Figure 2E). The major differences from an event-related design with pre-adaptation block are that (1) the pre-adaptation phase is directly followed by the classic event-related design while in studies with a pre-adaptation block the block can be presented long before the actual event-related experiment, (2) studies using a pre-adaptation block focus on more cognitive effects related to semantic priming, while studies employing an pre-adaptation phase are more interested in perceptual adaptation phenomena elicited with these longer adaptation phases, and (3) in the event-related design with pre-adaptation phase, the adaptor is repeated before every test stimulus ('topping-up stimulus'). Although topping-up stimulus and test stimulus immediately succeed each other, this design type is thought to reveal mid- to long-term adaptation effects dependent on the pre-adaptation phase (Krekelberg et al., 2005). This type of design was adopted by several investigators to study $\mathrm{fMRI}$ adaptation in early visual cortex to orientation (Fang et al., 2005; Engel, 2005; Jurcoane et al., 2007; Larsson et al., 2006; Montaser-Kouhsari et al., 2007) and color (Engel, 2005; Engel and Furmanski, 2001). To our knowledge, there are only two studies that employed a pre-adaptation phase in the study of higher-level vision such as the perception of faces (Fang et al., 2007; Ng et al., 2006).

In the case of orientation-dependent fMRI adaptation, the event-related design with a pre-adaptation phase has proven to be the only design capable of detecting orientation-dependent fMRI adaptation in V1. Attempts to detect adaptation effects to oriented gratings in V1 using classic event-related designs failed (Boynton and Finney, 2003; Murray, Olman et al. 2006). In a thorough investigation, Fang and co-workers (2005) directly compared the classic event-related design with the event-related design with pre-adaptation phase in the course of a study on orientation tuning in V1. They were able to reliably find orientation-dependent $\mathrm{fMRI}$ adaptation in $\mathrm{V} 1$ as well as in visual areas V2, V3, V3A, and hV4 when using the event-related design with a pre- 
adaptation phase of $20 \mathrm{~s}$ followed by topping-up-stimuli of $5 \mathrm{~s}$ and test stimuli of $1 \mathrm{~s}$ presentation duration. Without the pre-adaptation phase and with short presentation durations of the adaptor stimulus for $1 \mathrm{~s}$, however, they were only able to reveal orientation-dependent fMRI adaptation in V3A and hV4 (Fang et al., 2005). One possible explanation might be that primary or early visual cortex is less susceptible to adaptation in general. Interestingly however, three research groups (Kourtzi and Huberle, 2005; Murray, Olman et al., 2006; Weigelt et al., 2007b) were able to demonstrate spatially selective $\mathrm{fMRI}$-adaptation effects - that is fMRI-adaptation effects to retinotopic position - in V1 with classic rapid event-related designs.

In the case of $\mathrm{fMRI}$ adaptation to color, the studies so far (Engel, 2005; Engel and Furmanski, 2001) only used pre-adaptation phases plus rather long presentation durations of the adaptor stimulus. The authors demonstrate fMRI adaptation in V1 to repeated red-green gratings as well as to repeated black-white gratings while cross-adaptation (red-green/black-white or black-white/red-green) was clearly weaker. Because these were the only studies on color adaptation, it is unclear whether adaptation to color in V1 can be detected with a classic event-related design.

Fang and colleagues compared the classic event-related design with the eventrelated design with pre-adaptation phase in a study on face perception (Fang et al., 2007). They investigated the effect of viewpoint on face representations. Most importantly, the two different experimental designs revealed different viewpoint effects on adaptation. With the event-related design with pre-adaptation phase face-selective areas showed a graded viewpoint tuning, i.e., strongest adaptation for identical faces, less adaptation for 30 degrees rotation and even less adaptation for 90 degrees rotation. With the classic event-related design, the same regions showed strongly viewpoint-selective $\mathrm{fMRI}$ adaptation, that is adaptation for identical faces, but not for 30 degrees or 90 degrees of rotation (Fang et al., 2007). The opposite effect was observed in parahippocampal cortex: Epstein and colleagues (2005) found viewpoint-dependent fMRI-adaptation effects with a classic event-related design, whereas they observed viewpoint-invariant fMRI-adaptation effects using an event-related design with preadaptation. In summary, also in higher-level visual processing such as the perception of faces or places, the use of different designs might unravel different underlying neuronal processes.

\section{Event-related design with intermixed presentation}

During an event-related design with intermixed presentation, stimuli are presented in rapid succession. Some of the stimuli are repeated, some will occur only once. The repetition delay between the first presentation of the stimulus and its repetition is variable (Figure 2F). It can vary between $0 \mathrm{~s}$ (immediate repetition) and several seconds up to minutes. It is important to note that other stimuli are presented during the delay phase; and that the delay is not simply filled with a fixation period. One of the studies that applied an event-related design with intermixed presentation delays is a classical study by Henson and colleagues (2000). They presented familiar and unfamiliar faces and symbols and found fMRI-adaptation effects for familiar faces and symbols in ventral visual areas, while unfamiliar faces and symbols led to repetition enhancement (Henson et al., 2000). An advantage of this design type is that it allows to address the question whether repetition-related adaptation effects depend on repetition lag. 
It should be noted, however, that while some studies using an event-related design with intermixed presentation explicitly tested lag effects on adaptation (Henson 2000; 2004; Reber et al., 2005; Sayres and Grill-Spector, 2006) others did not (Henson et al., 2002; 2007; Ishai et al., 2004; Johnson et al., 2004; Turk-Browne et al., 2006; Vuilleumier et al., 2002; Yi and Chun, 2005; Williams et al., 2007).

In summary, it seems that the occurrence or non-occurrence of fMRI-adaptation effects critically depends on the interaction between design, visual area, and visual feature under study. This is an interesting first hint that although with every experimental design reported here one is able to elicit fMRI-adaptation effects, they might not all reflect the same underlying neuronal effect. Beyond the varieties in experimental design per se, the major difference between $\mathrm{FMRI}$ adaptation studies is in the repetition delay between the first presentation of a stimulus and its repetition. Shortest delays occur in classic event-related designs, while the longest delays were employed in event-related designs with pre-adaptation blocks.

\section{Stimulation parameters}

In the following paragraphs, we will report studies that investigated the effect of different stimulation parameters on $\mathrm{fMRI}$ adaptation. More precisely, we will focus on effects of repetition delay - the delay between the first stimulus (adaptor) and second stimulus (test) - and stimulus duration. We report studies that investigated the effects of delay and stimulus duration within one experimental design. For easy comparison, we converted the reported repetition delay measurements (stimulus onset asynchronies, inter pair interval) into measurements of inter stimulus interval (ISI), that is the time between the offset of one stimulus and the onset of the following stimulus. Thus, when we refer to repetition delay, this equals ISI.

\section{Repetition delay}

Several studies have investigated the effect of repetition delay on $\mathrm{fMRI}$-adaptation effects. First, we discuss short repetition delays that range from 0 to 7 seconds. Second, we cover studies that investigated middle (8 seconds to minutes) to long (minutes, hours, days) delays. Finally, we report on three studies that directly compared short with middle and long repetition delays.

\section{Short repetition delays (0-7 s)}

Boynton and Finney (2003) investigated orientation-specific fMRI-adaptation effects in early visual areas using ISIs that ranged from $125 \mathrm{~ms}$ to $7 \mathrm{~s}$. They demonstrated orientation-specific fMRI adaptation in areas V3 and V4 (Boynton and Finney, 2003). They did not perform an explicit statistical test to reveal differences of fMRI-adaptation effects with respect to repetition lag. However, there is evidence (Figure 5, Boynton and Finney, 2003), that they observed the effect across all repetition delays.

Huettel et al. (2004) studied direction-selective fMRI-adaptation effects using six ISIs between $1 \mathrm{~s}$ and $6 \mathrm{~s}$. They found clear fMRI-adaptation effects in lateral temporaloccipital and peri-calcarine cortex for repeated gratings moving in the same direction 
in contrast to gratings moving in opposite directions for all delays. Direction-selective fMRI adaptation was strongest with short ISIs.

Murray, Olman et al. (2006) presented arrays of broadband elements (disks that were half black and half white), in which elements were repeated either at the same or a different position. Using two different ISIs of $100 \mathrm{~ms}$ and $800 \mathrm{~ms}$ they investigated the effect of repetition delay on spatially-specific fMRI adaptation. They observed significant fMRI-adaptation effects for the repetition of the exact array (without a position change of the elements) in early visual areas V1, V2, V3 for both ISIs. No area showed a significant effect of ISI.

Intermediate repetition delays ( $8 \mathrm{~s}$ - minutes, but within a single scanning session) Henson et al. (2000) used an event-related design with intermixed presentation. They presented familiar and unfamiliar faces and symbols and the repetition lag ranged from $8 \mathrm{~s}$ to $20 \mathrm{~min}$ ( 1 to 147 intervening stimuli, median 45). They identified a right fusiform region that showed a significant interaction between familiarity and repetition lag for both faces and symbols: The response to the repetition of a familiar stimulus increased with lag while the response to the repetition of an unfamiliar stimulus decreased (Henson et al., 2000). The authors concluded that the processes that underlie the observed repetition effects in fusiform cortex decay over minutes.

Long repetition delays (hours, days)

Van Turennout and co-workers (2000) investigated intermediate (30 s) and long-lag (3 days) repetition delays. They found $\mathrm{fMRI}$-adaptation effects for intermediate and long repetition delays for both nameable and nonsense objects in occipitotemporal cortex. They also found fMRI-adaptation effects for both repetition delays in inferior frontal cortex but only for nameable objects. Interestingly, the adaptation was strongest in occipitotemporal areas for the intermediate repetition delay and in frontal regions for the long repetition delay (van Turennout et al., 2000). In a second study, van Turennout and colleagues (2003) further investigated the temporal evolution of those repetition effects, this time applying three different long repetition lags ( 1 h, $6 \mathrm{~h}$, and 3 days).Van Turennout et al. (2003) introduced the number of repetitions as another independent variable. They were thus able to investigate interaction effects between repetition lag and number of repetitions (van Turennout et al., 2003). Again, fMRI-adaptation effects were observable in occipitotemporal regions as well as in frontal cortex. There was no interaction effect between repetition time and number of repetitions in occipitotemporal regions: the $\mathrm{fMRI}$ signal decreased with number of repetitions, but the amount of $\mathrm{fMRI}$ adaptation to repeated objects was the same across the three long repetition lags. In contrast, in left inferior frontal cortex, multiple exposures led to a larger fMRIadaptation effect only with long repetition lags (van Turennout et al., 2003). Those results point towards two possibly different adaptation effects in visual and frontal cortex. In sum, van Turennout et al. $(2000 ; 2003)$ showed that fMRI-adaptation effects in occipitotemporal cortex favor intermediate repetition lags, but can also be reliably observed at several long lags (hours, days) and that those adaptation effects are influenced by the number of repetitions. 
Mixed repetition delays

Soon et al. (2003) compared a short ISI of 3 s to an intermediate ISI of 6 s. They presented unfamiliar faces and found $\mathrm{fMRI}$ adaptation to repeated faces in fusiform cortex for both ISIs. They detected a trend towards stronger fMRI adaptation for an ISI of $3 \mathrm{~s}$ in posterior fusiform gyrus (Soon et al., 2003).

Henson and colleagues (2004) investigated the effect of lag on fMRI adaptation within an event-related design with intermixed presentation. They compared ISIs of $780 \mathrm{~ms}$ (short), $2260 \mathrm{~ms}$ (short, with one intervening stimulus), $2260 \mathrm{~ms}$ (short, with no intervening stimulus) and $96 \mathrm{~s}$ (intermediate) (Henson et al., 2004). The main differences were found in object-selective regions in ventral occipital and temporal cortices, but also prefrontal areas: They detected fMRI-adaptation effects for repeated objects, where the size of adaptation decreased with increasing lag. Particularly, parahippocampal and lateral inferior occipital regions demonstrated a repetition-lag interaction.

We conclude that fMRI-adaptation effects in visual areas are observable even with very short delays $(100 \mathrm{~ms})$ and that they seem to be strongest for short or intermediate lags. In frontal areas, however, fMRI-adaptation effects occur also with very long repetition lags. Again, as in the case of the experimental design, we conclude that by using different repetition lags one might tap into different neuronal adaptation effects in a single brain area and that the relationship between fMRI-adaptation effects and repetition lag varies substantially across brain regions. However, most importantly, it seems that $\mathrm{fMRI}$ adaptation is able to detect various repetition effects across large time scales.

\section{Stimulus duration}

As described above, the duration of the adaptor stimulus has a high impact on orientation-selective fMRI-adaptation effects in primary visual areas when comparing different experimental designs. However, to our knowledge only one article investigated the influence of stimulus duration on $\mathrm{fMRI}$-adaptation effects directly. Zago and colleagues (2005) presented familiar everyday objects for 40 to $1900 \mathrm{~ms}$ followed by a mask of 1960 to $100 \mathrm{~ms}$. The test stimulus was then presented for $500 \mathrm{~ms}$. fMRIadaptation effects in object-related visual regions increased with increasing stimulus duration, peaking at $250 \mathrm{~ms}$, and decreased with longer exposures. These results may reflect different neuronal adaptation effects for short versus long stimulus durations. Unfortunately, the findings are confounded with varying repetition lags (as can be seen in the presentation durations of the mask), that is, the shorter the stimulus duration of the adaptor stimulus, the longer the mask and thus, the longer the repetition lag. Thus, based on this study a clear-cut interpretation of the influence of stimulus duration on adaptation is not possible.

\section{Number of repetitions}

Sayers and Grill-Spector (2006) demonstrated that fMRI-adaptation effects increased with the number of successive repetitions reaching a plateau at five repetitions. They tested up to eight repetitions of animal pictures and found this effect in LOC. Reber et al. (2005) found fMRI-adaptation effects to the second presentation (repetition) in a number of brain regions that displayed no further reduction with the following repetitions. Only some regions in the right fusiform gyrus and inferior temporal lobe exhib- 
ited linearly increasing $\mathrm{fMRI}$ adaptation responses for the second to the eighth presentation. A study by van Turennout and colleagues (2003) found also increasing fMRI adaptation with increasing number of repetitions in ventral visual cortex. In summary, brain regions in the ventral visual pathway seem to exhibit increasing $\mathrm{fMRI}$-adaptation effects with increasing number of repetitions, while other brain regions do not show further adaptation beyond the first repetition. This is a further hint that the underlying adaptation mechanisms might differ across brain regions.

\section{Visibility/Awareness}

There is evidence that repetition priming can occur without conscious perception of the primes (Tulving and Schacter, 1990). On the other hand, recent psychophysical experiments on low-level vision have suggested that certain types of adaptation depend on awareness of the presented stimuli (Blake et al., 2006). This section is devoted to studies that manipulated the visibility of either the adaptor stimulus or the test stimulus or both and looked for consequences on the fMRI-adaptation effect to repeated stimuli. Furthermore, we also report on studies that used illusory stimuli to see whether they also elicit fMRI adaptation.

Using a combination of forward and backward masking, Eddy and co-workers (2007) investigated fMRI-adaptation effects to invisible stimuli. In a classic event-related design they presented two objects, where the adaptor image was preceded and followed by a masking stimulus. Stimulus presentation times were short. Eddy et al. (2007) found fMRI-adaptation effects for repeated identical objects in several visual areas (fusiform gyrus, occipitotemporal regions). Furthermore, a region of the posterior inferotemporal gyrus showed even an fMRI-adaptation effect for repeated exemplar objects (two different cats). The authors conclude that areas of the ventral pathway are sensitive to image repetitions even when the adaptor stimulus is effectively masked.

Using an event-related design with pre-adaptation block, James and Gauthier (2006) investigated fMRI-adaptation effects for masked stimuli. They first presented object stimuli at full contrast in a localizer experiment (pre-adaptation block). Several minutes later those objects were repeated (among new objects) within an event-related scan. This time, object stimuli were shown at three different levels of contrast embedded in random noise with backward masking. James and Gauthier found fMRIadaptation effects to repeated objects only when subjects reported having been successful in identifying those objects (James and Gauthier, 2006). In contrast, they found an enhanced signal to repeated objects that could not be identified by the subjects.

Turk-Browne and colleagues (2007) manipulated the contrast of scene stimuli in a slow event-related design. They found fMRI-adaptation effects for the repetition of identical high-contrast scenes in comparison to different high-contrast scenes in the parahippocampal place area. Furthermore, they found fMRI enhancement for the repetition of identical low-contrast scenes in comparison to different low-contrast scenes in the same area (Turk-Browne et al., 2007).

In summary, Eddy et al. (2007) convincingly demonstrated that stimuli that are presented, but not perceived (due to effective masking) produce fMRI-adaptation effects in the LOC. A few studies investigated the inverse phenomenon:visual illusions, in 
which stimuli are perceived, but not presented. Montaser-Kouhsari et al. demonstrated fMRl-adaptation effects to illusory lines in early as well as late visual areas (MontaserKouhsari et al., 2007; Weigelt, 2007). In our own study we report on a cortical network of areas showing fMRI-adaptation to illusory, rotating objects (Weigelt et al., 2007a). Furthermore, we also demonstrate $\mathrm{fMRI}$-adaptation effects to perceptually completed shapes in ventral visual cortex (Weigelt et al., 2007b).

Thus both, stimuli that are not perceived although presented and stimuli that are perceived although not presented can lead to fMRI-adaptation effects. However, as soon as stimuli are degraded in contrast, as tested by James and Gauthier (2006) and Turk-Browne and colleagues (2006), those stimuli elicit fMRI enhancements in the respective areas. Since both later studies also used masking procedures, it is not clear how this might have affected the degradation of the stimuli. A possible explanation for these results is that repetition-related decreases occur if the first stimulus can be successfully processed by the visual system (that does not necessarily involve conscious perception). As soon as the first stimulus is not identifiable (due to degradation), the repetition of this stimulus might however support recognition. This extra processing that eventually leads to recognition is reflected in the repetition enhancement.

\section{Attention}

Attention enhances the efficiency of stimulus processing. Studies have shown that this effect is subserved by an enhanced selectivity of the neuronal population representing that stimulus (Spitzer et al., 1988). Since adaptation can be seen as a marker for sensitivity/selectivity, there should be a direct influence of attention on the amount of adaptation.

The first study that investigated the influence of attention on fMRI adaptation was conducted by Murray and Wojciulik (2004). Based on well-established fMRI-adaptation effects to shapes in the LOC the authors presented novel objects to their subjects during scanning. In a classic event-related design, one object could be followed either by a completely identical stimulus or by a stimulus identical in shape but rotated in plane. Attention was manipulated by instructing the subjects either to perform a task on the objects or a task on the fixation dot. Murray and Wojciulik (2004) observed a significant effect of rotation with decreasing amounts of adaption for increasing rotation angles in the LOC. Most importantly, they found a significant task-by-rotation interaction, indicating that attention to the objects in comparison to attention to the fixation point enhances the rotation-dependent selectivity (Murray and Wojciulik, 2004). In our own study (Weigelt et al., 2007a) we employed a similar attentional manipulation as Murray and Wojciulik. We found that fMRI-adaptation effects to illusory objects can only be observed if subjects are engaged in tasks that draw their attention towards the objects. A demanding center task abolished fMRI-adaptation effects in a network of cortical areas. However, it is hard to tell whether those effects found by Murray and Wojciulik and us rest upon spatial attention (attending to the complete object or attending to the small fixation spot) or object-based attention, because the manipulation of attention is confounded by the use of two completely different tasks. 


\section{Spatial attention}

Eger and colleagues tested the effect of spatial attention on fMRI adaptation (Eger et al., 2004b). They presented different objects to the left and the right of the fixation cross, followed by a single object at the center of the screen that could be an identical repetition of one of the first objects or a mirror image of one of the first objects. The modulation of spatial attention was achieved by instructing subjects to attend to either the left or the right side (while keeping central fixation). Eger et al. (2004b) found that only attended objects produced reliable fMRI-adaptation effects in lateral occipital and fusiform regions. Further confirmation that fMRI adaptation in ventral visual cortex is strongly dependent on spatial attention to the stimulus comes from a study by Henson and Mouchlianitis (2007). They showed subjects face and house stimuli on the left and right side of fixation and instructed them to attend either left or right. Again, reliable fMRI-adaptation effects were only found for stimuli that were attended. Moreover, those effects were category-specific in the sense that they were exclusively found for faces in the fusiform face area (FFA) and for houses in the parahippocampal place area (PPA) (Henson and Mouchlianitis, 2007).

\section{Object-based attention}

Apart from this strong influence of spatial attention on fMRI-adaptation effects, the influence of object-based attention was studied as well in two elegant papers by $\mathrm{Yi}$ and colleagues (Yi and Chun, 2005; Yi et al., 2006). Both studies made use of overlapping scene and face stimuli that were created by flickering a scene and a face image at a very high rate (the flicker itself was perceptually not resolvable). During scanning, subjects were asked to attend either to the scenes alone or attend to faces alone. In the PPA, fMRI-adaptation effects occurred only for attended repeated scenes, but not for ignored repeated scenes or attended repeated faces (Yi and Chun, 2005; Yi et al., 2006). In the FFA, fMRI-adaptation effects occurred only for attended repeated faces, but not for ignored repeated faces or attended repeated scenes ( $Y i$ and Chun, 2005). Interestingly, two different experimental designs were used: in their first experiment, $\mathrm{Yi}$ and Chun employed an event-related design with intermixed presentation ( $\mathrm{Yi}$ and Chun, 2005), while in the second experimental series they made use of a block design with different numbers of repetitions ( $\mathrm{Yi}$ et al., 2006). The effect of object-based attention was the same across experimental designs. A similar approach in a study of objectbased attention was taken by Vuilleumier et al. (2005). Again overlapping stimuli were created. Attended repeated objects elicited fMRI-adaptation effects in various occipitotemporal brain regions as well as in frontal cortex.

\section{Aftention effects on fMRI adaptation in early visual areas}

Interestingly, most studies that searched for fMRI-adaptation effects in early visual areas, especially primary visual cortex, used demanding center tasks to draw subjects' attention to the center of the screen (to prevent eye movements). With the appropriate experimental design involving longer pre-adaptation phases orientation-dependent fMRI-adaptation effects were observable in V1 (Fang et al., 2005; Jurcoane et al. 2007; Larsson et al., 2006; Montaser-Kouhsari et al.,2007) although subjects did not explicitly attend the oriented stimuli. In a recent experiment, Liu et al. (2007) however, investigated the influence of feature-based attention on orientation-selective fMRI adapta- 
tion in human primary visual cortex in more detail. They used composite stimuli:a double grating composed of two differently oriented gratings. Subjects were instructed to attend to one grating/one orientation only. In every area that they investigated (V1, V2, V3, hV4, LO1, LO2, V3A/B, V7), Liu et al. (2007) found an orientation-dependent fMRIadaptation effect for the attended grating in contrast to the unattended grating. Interestingly, they computed correlation coefficients between behavioral measures of orientation-dependent adaptation (tilt aftereffect) with the attentional effect on the fMRI-adaptation signal for every visual area and found that there was a significant correlation only for area V1. Liu et al. (2007) speculate that if attention selectively modulates the response of neurons representing specific features, then the most efficient modulation would occur on the level in which neurons show the highest functional specialization for this feature (in this case in V1 for orientation). We can summarize that orientation-dependent fMRI-adaptation effects in early visual cortex can be observed without attention being directed towards the stimuli, but that if attention is directed to the stimulus, this enhances the fMRI-adaptation effect even more.

Additional evidence that fMRI-adaptation effects in early visual areas are observable independent of the attentional focus, comes from a study by Murray, Olman and colleagues (2006). Testing spatially specific adaptation - that is adaptation to retinotopic position - Murray and colleagues did not find any differences for V1 whether or not the stimuli were attended. They used sparsely distributed, oriented black-andwhite disks in a classic event-related design. A stimulus array was followed either by the identical array or by a different array in which individual elements were displaced by 1 degree visual angle. Subjects performed either a same/different task on the two stimulus arrays or a demanding center task. Spatially-specific fMRI-adaptation effects for the identical array were observed in early visual areas V1, V2, and V3 independent of the task being performed (Murray, Olman et al., 2006).

We conclude that fMRI-adaptation effects in higher visual areas seem to be highly susceptible to attentional modulation. fMRI-adaptation effects in early visual areas are less dependent on the attentional focus, i.e., they are also observable without attention.

\section{Comparing $f M R I$ and neural adaptation}

In conclusion, $\mathrm{fMRI}$-adaptation effects can be reliably elicited with various experimental designs. However, there seem to be differences whether a certain design will reveal adaptation effects across brain regions in general, across visual areas in particular and even within a visual area across neuronal subpopulations coding for different visual features. We therefore hypothesize, that the observed fMRI-adaptation effects reflect various neuronal adaptation mechanisms dependent on the experimental design and the stimulation parameters employed. That there are indeed various adaptation mechanisms at the level of single neurons or cell assemblies has been shown (Kohn, 2007).

Still, one might argue that in the case of neuroimaging the observed differences between experimental designs do not reflect differences in the neural mechanisms, but are attributable to non-linear effects, e.g., in the neurovascular coupling (Boynton and Finney, 2003; Huettel et al., 2000). However, two electrophysiological studies sup- 
port the idea that the use of different experimental designs also has a strong impact on the neuronal adaptive mechanisms. Priebe and Lisberger (2002), Priebe and colleagues (2002) and Kohn and Movshon (2003) addressed the question whether adaptation of cells in the motion-sensitive region (MT) of the macaque might be inherited from primary visual cortex. They adapted one portion of the receptive field of an MT neuron and assessed the transfer of adaptation to another portion of the receptive field. A transfer of adaptation from one location to the other would speak in favor of an adaptation mechanism that has to be intrinsically computed in MT or signalled via feedback from higher areas, while no transfer of adaptation would indicate that the effects were based on input from early visual areas. Surprisingly, Priebe and colleagues found a transfer of adaptation, while Kohn and Movshon did not (Kohn and Movshon, 2003; Priebe et al., 2002). The discrepant results are most likely caused by the use of different adaptation approaches. Priebe et al. used brief presentations of stimuli (64 ms stimulus durations and ISIs ranging from 0 to $256 \mathrm{~ms}$ ), while Kohn and Movshon investigated adaptation effects that occured after prolonged stimulation (pre-adaptation phase of $40 \mathrm{~s}$ plus top-up adaptors of $5 \mathrm{~s}$ ). Furthermore, the two studies differed also in their stimulus material: Priebe et al. (2002) used random dots while Kohn and Movshon (2003) used sine-wave gratings. We therefore conclude that differences in experimental design and/or stimulus material might reveal different neuronal adaptation mechanisms. How these mechanisms relate to each other and how they are generated in the cell or cell assemblies remains to be answered. Furthermore, the question whether adaptation in higher visual areas might be inherited from earlier visual areas or might be modulated by other areas is still open. Importantly, the fact that different experimental designs lead to different adaptation effects at the neuronal level might be used as evidence that the observed differences at the hemodynamic level indeed reflect neuronal differences.

In contrast, a study by Sawamura and coworkers (2006) investigated neuronal adaptation mechanisms with two different approaches and found no difference between the designs. Interestingly, this research group employed experimental designs derived from typical fMRI adaptation studies. They used a classic event-related design and a block design with different numbers of repetitions. Sawamura et al. studied the repetition-related activity of IT cells in macaque monkeys; stimuli comprised different familiar objects. Most importantly, they did not find any differences between the two experimental designs, both approaches reliably elicited neuronal adaptation to the repetition of objects in IT cells (Sawamura et al., 2006). Thus, it seems that prolonged adaptation phases as were used by Kohn and Movshon (2003) are a special case and might involve different mechanisms from short-lag adaptation.

Further support for this hypothesis stems from two different lines of evidence: First, it has been shown that prolonged adaptation does not only influence firing rates, but also the tuning of neurons. Kohn and Movshon (2004) studied the direction tuning of cells in areas MT and V1 of the macaque and found that adaptation of near-preferred direction caused a tuning shift toward the adapted direction for MT cells but not for V1 cells (Kohn and Movshon, 2004). Furthermore, this 'attractive' shift of MT cells stands in direct contrast to the'repulsive' shift of $\mathrm{V} 1$ cells that has been observed, e.g., for orientation (Dragoi et al., 2000; Müller et al., 1999). Beyond showing that prolonged adaptation has a major impact on a cell's tuning curve, this study also indicates that different 
cortical regions may adapt to repetitive input in distinct ways. Second, Tolias and coworkers (2005) found that long adaptation periods lead to strong direction-selective adaptation in V4v of the macaque - an area that is normally not seen to comprise cells that are strongly direction-selective. This finding is in line with the results by Fang et al. (2005) and Epstein et al. (2005) reviewed above.

In summary, prolonged adaptation targets both early and late visual cortex and has a strong influence not only on firing rates but also on the tuning of cells. We hypothesize that some of the effects observed during prolonged adaptation in higher visual areas are inherited from early visual areas. In contrast, short-lag adaptation effects can be detected with much more ease in higher visual areas. We hypothesize that these short-lag adaptation effects mark an intrinsic adaptive behavior of cells within a cortical region or may be elicited by feedback connections (McMahon et al., 2007; Priebe and Lisberger, 2002). We therefore argue that $\mathrm{fMRI}$ adaptation does not refer to a single process but rather to a number of possible neuronal adaptation mechanisms.

Finally, we want to emphasize the difficulty of interpreting null effects in fMRI adaptation designs. Based on our current findings on the impact of experimental design, stimulus duration, repetition lag, visibility, and attention, null effects in $\mathrm{FMRI}$ adaptation studies might be due to numerous factors and their interactions, and are thus not easily interpretable. Further empirical evidence is needed to gain a complete picture of the different effects of stimulus repetition on the population and neuronal level.

\section{Functional role of adaptation}

A fundamental and still open question is the functional role of adaptation. In what follows we discuss three possible roles of adaptation: (1) adaptation being the neuronal correlate of behavioral priming, (2) adaptation enhancing stimulus selectivity and (3) adaptation mediating memory formation.

FMRI adaptation was first thought to be the neuronal correlate of behavioral priming, since a number of studies investigating behavioral priming phenomena found fMRI adaptation in parallel with reaction-time facilitation (Buckner et al., 1998; Henson, 2003; Henson and Rugg, 2003; Maccotta and Buckner, 2004; Naccache and Dehaene, 2001; Schacter et al., 1998; 2004). However, some recent studies showed a dissociation between behavioral priming and fMRI-adaptation effects (Bunzeck et al, 2006; Ganel et al., 2006; Sayres and Grill-Spector, 2005; Xu et al, 2007). Thus, the concept of adaptation as the neuronal correlate for behavioral priming has been challenged. Furthermore, using transcranial magnetic stimulation (TMS), Wig et al. (2005) showed that behavioral priming was absent and that fMRI-adaptation effects in frontal cortex but not in ventral visual areas were abolished, when TMS was applied to the frontal cortex. Those studies point towards a more complex picture of the relationship between behavioral priming and $\mathrm{fMRI}$ adaptation such that $\mathrm{fMRI}$ adaptation in visual areas does not necessarily reflect behavioral priming, but possibly fMRI adaptation in frontal cortex does (Schacter et al., 2007).

Secondly, adaptation has been claimed to enhance stimulus selectivity (Tolias et al., 2005). In this respect a recent study by Sawamura and colleagues (2005) is of great importance. Neurons in IT cortex of the macaque were recorded in a single-cell study. 
Sawamura et al. selected two stimuli ( $A$ and $B$ ) to which the neuron responded equally well (with the same high amount of spiking) and other stimuli to which the neuron responded weakly (C). Those stimuli were tested in an adaptation experiment. The main finding of Sawamura and coworkers was that the repetition of the same stimulus (A-A) always elicited a stronger adaptation than the presentation of $A-B$ or $B-A$, although the initial responses were the same for A and B (Sawamura et al., 2005). In other words, stimulus selectivity based on adaptation was much stronger than the selectivity based on the initial response of the neuron to the stimulus. This finding was independent of the experimental design employed. Further support for an adaptation-dependent selectivity stems from the work of Tolias et al. (2001; 2005). Based on their neuroimaging and electrophysiological investigations, Tolias and coworkers propose that adaptation may finally increase the sensitivity of neurons to detect changes in the environment.

A third functional role of adaptation was recently proposed on the basis of a neuropsychological case study. Williams and coworkers (2007) examined a rare case of developmental prosopamnesia. The patient reported a lifelong difficulty in recognizing people by their faces and extensive behavioral testing revealed that the patient is severely impaired in memorizing unfamiliar faces and relies on a feature-matching strategy when judging facial identity. However, the patient was able to develop familiarization with faces over months (e.g., famous faces and faces of family members) and her discrimination between familiar and unfamiliar faces was very good (Williams et al., 2007). Williams and colleagues employed an event-related adaptation design with intermixed presentation to study the functioning of the patient's fusiform face area. They presented familiar and unfamiliar faces and places while the subject was instructed to passively view the images. $\mathrm{FMRI}$ adaptation to the repetition of familiar and unfamiliar places was observed in PPA (both in the patient and in a group of control subjects). Most interestingly, the right FFA displayed fMRI adaptation to familiar faces but not to unfamiliar faces in the patient, while both familiar and unfamiliar faces caused adaptation in control subjects (William et al., 2007). The authors conclude that repetition suppression has revealed a neuronal marker for the striking deficit in this case of prosopamnesia.

Further evidence comes from studies of elderly patients who suffer from cognitive impairments. Focusing on the hippocampus and parahippocampal gyri, Johnson et al. (2004) found clear fMRI-adaptation effects for repeated faces in a group of cognitively healthy, old subjects. However, no adaptation effect could be observed in the patients. A study by Lustig and Buckner did not find any evidence for impaired fMRI adaptation in a group of patients in an early stage of amnesia (Lustig and Buckner, 2004).

We conclude that further empirical evidence is needed to understand the phenomenon of adaptation. Since the phenomenon itself occurs at various levels - behavioral, cellular, network - a multimethod approach is highly desirable (Clifford et al., 2007). Interference studies using TMS (Wig et al., 2005) and neuropsychological case studies (Williams et al., 2007), but also pharmacological approaches (Bentley et al., 2003; Thiel et al., 2001;2002) might reveal a more detailed picture of the underlying mechanisms of adaptation in humans. The field of adaptation research will definitely profit from the combination of electrophysiological and neuroimaging techniques devoted to unravel the mechanisms of neurovascular coupling. This will increase the reliability, explanatory power, and the predictive validity of $\mathrm{FMRI}$ adaptation. 



\section{The cortical representation of objects rotating in depth}

The perception of motion provides valuable interpolations of the visual scene. This fundamental capacity of the visual system is evident in apparent rotation: by presenting only two images of an object rotated in space, a vivid illusion of a smooth apparent motion in three dimensions can be induced. The unseen interpolated rotation views are filled in by the visual system. In the present study, we identified the cortical network responsible for this filling-in process. We argue that cross talk between areas of the ventral and dorsal visual pathways promote the illusion of smooth apparent rotation. Most interestingly, the network represents the unseen object views. Using functional magnetic resonance adaptation, we are able to show that the cortical network selectively adapts to the illusory object views. Our findings provide strong evidence for cortical representations of three-dimensional rotating objects that are view invariant with respect to the rotation path. Furthermore, our results confirm psychophysical investigations that unseen interpolated rotation views can be primed by apparent motion. By applying functional magnetic resonance adaptation, we show for the first time cortical adaptation to unseen objects. Together, our neuroimaging study advances the understanding of the cortical mechanisms mediating the influence of motion on object processing.

Weigelt, S., Kourtzi, Z., Kohler, A., Singer, W., and Muckli, L. (2007). The cortical representation of objects rotating in depth. The Journal of Neuroscience, 27: 3864-3874. 


\section{Introduction}

In our natural environment, we frequently encounter objects in different rotated views. The perception of a rotating object might either be attributable to the object moving or to the observer moving. In both cases, different views of that object are revealed, whereas others become occluded. Kourtzi and Shiffrar investigated how different views of an object might be linked together by motion either in two dimensions (Kourtzi and Shiffrar, 1997) or in three dimensions (Kourtzi and Shiffrar, 1999). In the latter study, the authors made use of a visual illusion called 'apparent rotation' (Shepard and Judd, 1976). If two views of the same three-dimensional (3D) object are presented successively, they produce an illusion of rigid rotation: one object moving back and forth. Kourtzi and Shiffrar (1999) found that an apparent-rotation sequence of an object rotating in depth primed unseen views of that object. Most importantly, only views of that object were primed that lay on the rotation path of the object (interpolated positions). Views that lay outside the rotation trajectory (extrapolated positions) were not primed (Kourtzi and Shiffrar, 1997). These results were replicated in a recent study by McNamara et al. (2006). These studies provide evidence that apparent motion facilitates viewpoint-invariant representations of objects within the path of rotation.

The fact that motion primes unseen (interpolated) rotation positions suggests that the human visual brain represents these interpolated views. The goal of this study was to identify brain regions that are involved in the cortical representation of interpolated rotation positions. Using functional magnetic resonance imaging (fMRI), we aimed at localizing the motion-specific, viewpoint-invariant representations of rotating 3D objects. To find brain regions that represent unseen rotation positions, we made use of cortical adaptation effects that are observable using the fMRI-adaptation paradigm (Buckner et al., 1998; Grill-Spector et al., 1999; Tootell et al., 1998a) (for review, see GrillSpector et al., 2006; Krekelberg et al., 2006).

We conducted three fMRI-adaptation experiments using an apparent-rotation sequence as the adapting stimulus that was followed by test stimuli that were rotated to different positions. We found regions of the occipito-temporal cortex [functionally defined as the lateral occipital complex (LOC)] (Kourtzi and Kanwisher, 2000; Malach et al., 1995), the transverse occipital sulcus (TOS), the lateral occipital sulcus (LOS), and the superior parietal cortex (SPC) that demonstrated adaptation of activation for stimuli that lay on the rotation path compared with stimuli that lay off the path. A fourth fMRIadaptation experiment confirmed that these adaptation effects are truly based on the perceived motion and do not occur with static stimuli. Thus, we were able to identify a cortical network representing view-invariant representations of 3D objects rotating in depth that are motion specific. These findings advance our understanding of complex visual illusions and the cortical mechanisms mediating the influence of motion on object processing. 


\section{Materials and Methods}

\section{Subjects}

Twenty-nine healthy subjects participated in this study (5 in experiment 1, 10 in experiment 2,8 in experiment 3, and 6 in experiment 4). Subjects were students recruited from the University of Tübingen (experiment 1 ) and the University of Frankfurt (experiments 2-4). All subjects had normal or corrected-to-normal vision. The data from one subject had to be excluded because of inadequate slice positioning (experiment 1). Because of excessive head motion, the data of three subjects (one from experiment 2 , two from experiment 3 ) had to be excluded. Before participating in this study, all volunteers gave their informed written consent to the procedure in accordance with institutional guidelines and the Helsinki declaration (www.wma.net/e/ethicsunit/helsinki.htm).

\section{Stimuli}

Five different 3D objects were created using the BrainVoyager 2000 software package (Brain Innovation, Maastricht, The Netherlands). Stimuli were comparable to those designed by Shepard and Metzler (1971). Each object consisted of 10 solid cubes attached face to face to form a rigid arm-like structure with three to four right-angled joints. Each object was asymmetrical around any possible axis. The objects were chosen to be relatively unfamiliar and meaningless in overall shape. They were presented as grayscale images in front of a black background. Five different perspective projections were generated for each object by 30 deg rotation steps around the vertical axis. These five objects, respectively their perspective projections, formed a first sample of stimuli. To create a second sample, each object of the first sample was rotated by 180 deg around the horizontal axis (see Fig. 1 for examples).

From this sample, additional stimuli were created for experiment 4. By superimposing two different perspective projections of one stimulus, double stimuli were created (see Fig. 1a, experiment 4 for examples). The overlapping area of the two stimuli was made transparent (10\%) so that the two stimuli were perceived as individual objects.

\section{Visual stimulation}

Visual stimuli were delivered under computer control to a high-luminance liquid crystal display projector. The image was projected via a mirror onto a frosted screen that was positioned at the head end of the scanner (experiment 1) or directly projected on a screen that was fixed to the head coil (experiments 2 and 3). Subjects viewed both screens through a tilted mirror that was mounted onto the head coil. In experiment 4, stimuli were delivered via a video goggle system (VisuaStim Digital Glasses; Resonance Technology, Northridge, CA). Stimuli subtended $500 \times 500$ pixels, which resulted in a visual angle of approximately 8 deg.

Each experimental run began with a fixation point presented for $16 \mathrm{~s}$, then followed an experimental block consisting of 109 trials $4 \mathrm{~s}$ each (90 experimental trials, plus 18 fixation trials, plus one extra trial; see below). At the end of each run, a fixation point was shown for $8 \mathrm{~s}$. In summary, one experimental run lasted $7 \mathrm{~min}, 40 \mathrm{~s}$ (460 s). Subjects performed four to five experimental runs within one scanning session. Each experimental run had 18 trials per experimental condition. 
a

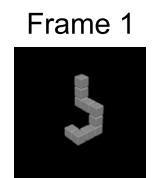

ISI 1

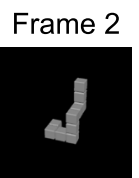

ISI 2

Test
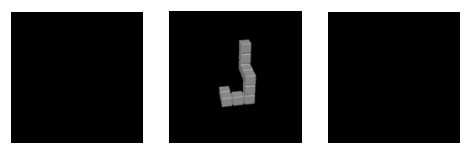

Experiment 4
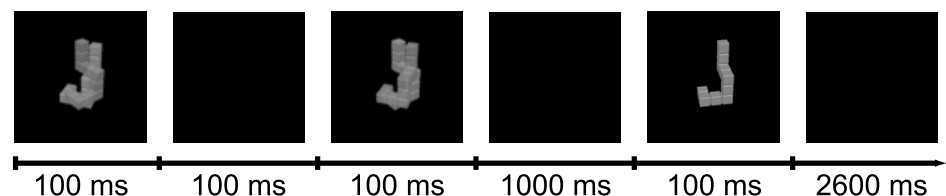

b

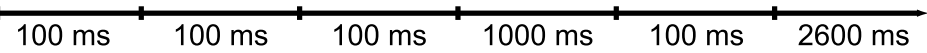

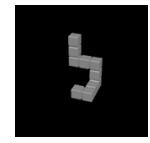

reverse extrapolated

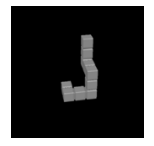

interpolated

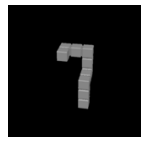

novel

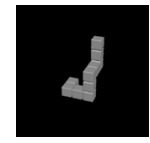

repeated

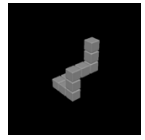

continuous extrapolated
C
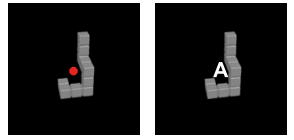

Exp. 1,2,4 Exp. 3

Figure 1. Experimental procedure. a, Schematic illustration of the experimental procedure demonstrating the timing of the experimental trial. An experimental trial began with the presentation of the apparent-rotation sequence. The first frame was shown for $100 \mathrm{~ms}$, followed by an interstimulus interval lasting $100 \mathrm{~ms}$ (ISI 1). Then, the second frame was presented for $100 \mathrm{~ms}$. After the apparent-rotation sequence, a blank screen was displayed for $1000 \mathrm{~ms}$ (ISI 2), followed by the test stimulus (Test), which was shown for $100 \mathrm{~ms}$. After the presentation of the test stimulus, the screen went black again for $2600 \mathrm{~ms}$ [intertrial interval (ITI)]. b, Perspective projections of one object from the stimulus set used in the experiment. Two stimuli constituted the apparent rotation sequence (frames 1 and 2). The type of condition is defined by the test stimulus. A test stimulus was a repetition of the second frame (repeated; orange), a stimulus rotated to an interpolated position (interpolated; red), a stimulus extrapolated in the direction of motion (continuous extrapolated; blue), a stimulus extrapolated in the reverse direction of the path of motion (reverse extrapolated; turquoise), or a novel stimulus, which was equivalent to the interpolated stimulus of the corresponding object of the second sample of stimuli (novel; green). Stimuli were created using BrainVoyager 2000 (Brain Innovation). c, Schematic illustration of the different tasks for the three experiments. In experiments 1, 2, and 4 (Exp. 1,2,4), subjects were instructed to maintain fixation on a red fixation point that was present during the entire trial, while performing different tasks on the objects. In experiment 1, subjects had to indicate via button press whether the test stimulus depicted the same object compared with the object of the apparent-rotation sequence or a different one (OIT). In experiment 2, subjects had to decide whether the test stimulus was rotated in the same direction of motion as the apparent-rotation sequence or in the other direction (DMT). In experiment 4, subjects had to indicate whether the test stimulus depicted a part of the double stimulus or not. In experiment 3 (Exp. 3), subjects performed an attention-demanding task on a randomly generated stream of letters and digits that was presented at $2 \mathrm{~Hz}$ at the center of the screen. They had to press a button whenever they detected a digit (ACT).

\section{Experiments 1-3}

An experimental trial began with the presentation of the apparent-rotation sequence (see Fig. 1a, experiments 1-3). The first frame was shown for $100 \mathrm{~ms}$, followed by an interstimulus interval lasting $100 \mathrm{~ms}$. Then, the second frame was presented for 100 
ms. After the apparent-rotation sequence, a blank screen was displayed for $1000 \mathrm{ms,}$ followed by the test stimulus, which was shown for $100 \mathrm{~ms}$. The test stimulus was a repetition of the second frame (condition,'repeated'), a stimulus rotated to a position halfway between the two apparent-rotation frames ('interpolated'), a stimulus extrapolated in the direction of motion ('continuous extrapolated'), a stimulus extrapolated in the reverse direction of the path of motion ('reverse extrapolated'), or a novel stimulus that was equivalent to the interpolated stimulus of the corresponding object of the second sample of stimuli ('novel'). See Figure $1 \mathrm{~b}$ for examples of the different test stimuli. After the presentation of the test stimulus, the screen went black again for 2600 ms (intertrial interval); then a new trial began. The direction of the apparent-rotation sequence was balanced across trials. The order of the presentation of the experimental trials was counterbalanced across runs per subject [i.e., trials from each condition (fixation included) were preceded (one trial back) equally often by trials from each other condition]. Because of this strategy, the first experimental trial (which had no history) had to be repeated at the end of the experimental block (extra trial). Taking all five experimental runs together, a full counterbalancing was achieved.

\section{Experiment 4}

The timing of an experimental trial as well as the experimental conditions was the same in experiment 4 compared with experiments 1-3. Only frame 1 and frame 2 differed. Instead of an apparent-rotation sequence, subjects were presented two double stimuli (see Fig. 1a, experiment 4). The new frame 1 was a superposition of the original frame 1 onto frame 2, and the new frame 2 was a superposition of the original frame 2 onto frame 1.This presentation abolished the perception of motion between frames 1 and 2; subjects reported to see the stimuli as flickering.

\section{Behavioral task}

In experiment 1, subjects had to indicate via button press whether the test stimulus depicted the same object compared with the object of the apparent-rotation sequence or a different one [object identification task (OIT)]. In experiment 2, subjects had to decide whether the test stimulus was rotated in the same direction of motion as the apparent-rotation sequence or in the other direction [direction of motion task (DMT)]. In experiment 4, subjects had to indicate whether the test stimulus depicted a part of the double stimulus or not. In all three experiments, subjects were instructed to maintain fixation on the red fixation dot, which was visible at all times during the entire experiment (see Fig. 1c). In experiment 3, subjects performed an attention-demanding task on a randomly generated stream of letters and digits (with a size of 0.8 deg visual angle) that was presented at $2 \mathrm{~Hz}$ at the center of the screen. They had to press a button whenever they detected a digit [attention control task (ACT)] (see Fig. 1c).

\section{Data acquisition}

Blood oxygenation level-dependent (BOLD) fMRI was performed on 3T Siemens Trio (experiments 1-3) and Allegra (experiment 4) scanners (both from Siemens, Erlangen, Germany) equipped with birdcage head coils at two different locations (experiment 1, University Clinics, Tübingen; experiments 2-4, Brain Imaging Center, Frankfurt). Scanning procedures were kept similar, and sequence parameters differed only slightly between experiments. A gradient-recalled echo-planar imaging sequence was used with 
the following parameters: number of slices, 11; repetition time (TR), 1000 ms; echo time (TE), 40 ms; flip angle (FA), 60 deg; field of view (FOV), 210; slice thickness, $5 \mathrm{~mm}$; in-plane resolution, $3 \times 3 \mathrm{~mm}^{2}$; gap thickness, $1 \mathrm{~mm}$ (experiment 1). The slices were oriented to reach a total coverage of the occipital and temporal lobes (see Fig. 2). In experiments $2-4$, the following parameters differed compared with experiment 1: number of slices, 16 ; TE, $30 \mathrm{~ms}$; slice thickness, $6 \mathrm{~mm}$. These parameters yielded a total coverage of the entire brain. Functional images were acquired in four to five experimental runs in a single session. Each run comprised the acquisition of 460 volumes. Stimulus presentation was synchronized with the fMRI sequence at the beginning of each run. Each scanning session included the acquisition of a high-resolution magnetization-prepared rapidacquisition gradient echo sequence for coregistration and anatomical localization of functional data [TR, $2000 \mathrm{~ms}$; TE, $4.38 \mathrm{~ms}$; FA, 15 deg; FOV, 240; voxel size, $1 \times 1 \times 1 \mathrm{~mm}^{3}$ (exp. 1);TR, $1240 \mathrm{~ms} ; \mathrm{TE}, 2.6 \mathrm{~ms}$; voxel size, 1 × 1 × 2 mm³ (exp.2-4)].

\section{Data preprocessing}

Data analysis and visualization was performed using the BrainVoyager QX software package (Brain Innovation, Maastricht, The Netherlands). The first four volumes of each event-related run were discarded to preclude T1 saturation effects. Preprocessing of the functional data included (1) 3D motion correction, (2) linear trend removal and temporal high-pass filtering at $0.01 \mathrm{~Hz}$, and (3) slice scan-time correction with sinc interpolation. For each subject, the functional and structural 3D data sets were transformed into Talairach coordinate space (Talairach and Tournoux, 1988). The recorded high-resolution anatomy of one subject of experiment 1 and of all subjects of experiments 2 and 3 were used for surface reconstruction, which included gray/white matter segmentation based on intensity values. The cortical surfaces were slightly smoothed, inflated, and flattened (using a manual cut through the calcarine sulcus).

For experiments 2 and 3, a spatial correspondence mapping between subjects brains was performed using the BrainVoyager QX cortex-based intersubject alignment tool. It has been shown that a cortical correspondence mapping substantially improves statistical analysis across subjects by reducing anatomical variability (Fischl et al., 1999; Goebel et al., 2006; Meienbrock et al., 2007; van Atteveldt et al., 2004). The procedure involved the reconstruction of the gray/white matter boundary of each individual hemisphere. The cortical surfaces were morphed into a spherical representation, and the hemispheres were then aligned based on the curvature information regarding the gyral/sulcal folding pattern. The target of the morphing procedure was a dynamical group average of all included hemispheres. This approach avoids the selection of a specific target hemisphere (Goebel et al.,2006). The correspondence mapping between the individual hemispheres was then used for the alignment of the functional data.

\section{Data analysis}

Our rapid event-related fMRI study used closely spaced trials, leading to a substantial overlap in the resulting hemodynamic responses. Nevertheless, under the assumption of linearity, the underlying hemodynamic responses can be assessed by deconvolution (Dale and Buckner, 1997). A deconvolution analysis estimates the hemodynamic response function for each trial on the basis of a general linear model (GLM). Twenty predictors were defined to cover the temporal extent of a typical hemodynamic re- 
sponse. Because of the hemodynamic lag in the BOLD-fMRI response, differences between conditions (as well as the peak in overall response) are expected to occur at a lag of several seconds after stimulus onset (Boynton et al., 1996; Cohen, 1997). On the basis of the deconvolved fMRI signal, we identified the peak points at lags 5-7 s after trial onset. To localize regions in the brain that show a significantly lower activation to the repeated stimulus in contrast to the novel stimulus, a deconvolution-based GLM was computed at this peak point (contrast rep < nov; 'basic adaptation effect'). The GLM included percent signal change normalization [i.e., the time course of a voxel or region of interest (ROI) is normalized in a way that the mean signal value will be transformed to a value of 100 and the individual values fluctuate around the mean as a percent signal deviations]. Thus, the reported GLM parameter estimates (weights) directly provide an estimate of the actual percent signal change. Additionally, we provide averaged parameter estimates across peak points ( $5-7 \mathrm{~s}$ after trial onset) and trials and subjects per condition as bar plots.

Multisubject statistical maps were thresholded using the false discovery rate (FDR) (Genovese et al., 2002), and q was set to 0.05 (experiments 1-3). For experiment 4, we performed a cluster-size thresholding to correct for multiple comparisons (Forman et al., 1995). We used a plug-in for BrainVoyager QX from F. Esposito (Brain Innovation, Maastricht, The Netherlands) that makes use of a Monte Carlo simulation (1000 iterations). Thus, the multisubject statistical map for experiment 4 is thresholded at $p<$ 0.05 , corrected for multiple comparisons. To reveal an adaptation effect for the critical, interpolated condition, we looked at the detailed response profiles by individual ROI-based-GLM analyses of the predefined regions. On each ROI, we performed five different pairwise comparisons: (1) contrasting interpolated versus novel (int < nov); (2) interpolated versus continuous extrapolated (int < conex); (3) interpolated versus reverse extrapolated (int < revex); (4) continuous extrapolated versus novel (conex < nov); and (5) reverse extrapolated versus novel (revex < nov). For ROI-based analyses, the data were normalized to percent signal change and corrected for serial correlation (Bullmore et al., 1996). We report t statistics and corresponding $\mathrm{p}$ values. Note that the first contrast (int < nov) is not independent of the contrast that defines the ROI (rep < nov) and is therefore only of descriptive use. Contrasts 2 and 3, however, are statistically independent of the ROI-defining contrast. For each ROI, we also extracted the eventrelated deconvolution time course of signal intensity (see Figs. 2-5) and provide GLM parameter estimates (see Figs. 3, 5; Additional Figs. 1, 2).

\section{Analysis for the comparison of experiments 2 and 3}

To reveal similarities and/or differences caused by the different tasks, we wanted to directly compare the results of experiment 2 (DMT) with results obtained in experiment $3(A C T)$. We therefore matched the number of subjects from experiment 2 by randomly selecting six subjects out of the total population of nine. We then applied the same statistical analysis as in experiments 1 and 2 for the subpopulation of experiment 2 and for experiment 3 including cortex-based intersubject alignment and GLM group analyses on the peak points of the averaged time course (contrast repeated vs novel, rep $<$ nov). On a low threshold of $p<0.05$ (uncorrected), we investigated the response profiles of the overlapping regions of the functional activation maps for experiments 2 and 3, conducting ROI-based analyses for each experiment, respectively 
(DMT, ACT). As in experiments 1 and 2, we computed five different contrasts for each ROI and each experiment: (1) interpolated versus novel (int < nov), (2) interpolated versus continuous extrapolated (int < conex), (3) interpolated versus reverse extrapolated (int < revex), (4) continuous extrapolated versus novel (conex < nov), and (5) reverse extrapolated versus novel (revex < nov). Again, for ROI-based analyses, the data were normalized to percent signal change and corrected for serial correlation. We report $t$ statistics and corresponding $p$ values. For each ROI and experiment, we also extracted the event-related deconvolution time course of signal intensity (see Fig.4) and provide GLM parameter estimates (see Additional Fig. 2).

\section{Eye movements}

We measured movements of the right eye of three subjects inside the magnet by pupil tracking under infrared illumination (MReyetracking system in conjunction with VisuaStim Digital Glasses, Resonance Technology; ViewPoint software, Arrington Research, Scottsdale, AR). The experimental setup was the same as in experiment 2; subjects were performing the DMT. Eye traces were analyzed using Matlab (The MathWorks, Natick, MA) and Statistica (StatSoft, Tulsa, OK). Processing involved linear trend removal of the horizontal and vertical eye-movement traces and detection of saccades and eye blinks exceeding $1.5 \mathrm{deg}$ of visual angle and a duration of $150 \mathrm{~ms}$. Eye movements that exceeded these limits were classified into blinks or saccades depending on a signal parameter that detected eye-lid closure (signal loss). The eye movements were assigned to the different stimulation conditions, and nonparametric statistics were performed to analyze whether there was a systematic change of eye-movement parameters between experimental conditions. The average number of saccades/blinks was $0.15 / 0.7$ per trial. There were no significant differences between conditions in the number of saccades $\left(X^{2}=6.515 ; \mathrm{df}=4 ; \mathrm{p}<0.164\right)$ or blinks $\left(X^{2}=8.133 ; \mathrm{df}=4 ; \mathrm{p}<0.087\right)$. Furthermore, we plotted individual eye positions for each condition and participant (two-dimensional fixation-density plots) (Additional Fig. 3). The brain imaging data were analyzed the same way as in experiments 1-4 up to the level where statistical contrasts were computed. Instead of first computing a basic adaptation contrast and performing ROI-based analyses to reveal the specific adaptation effects for the interpolated condition afterward, we used a conjunction analysis of the contrast rep < nov and the balanced contrast int $<$ (conex and revex). This procedure yielded two regions in the parietal cortex that demonstrated the specific adaptation profile. Additional Figure 4 displays the contrast map and corresponding deconvolved signal time courses. In summary, we replicated the specific fMRI-adaptation effects in this group of three subjects for which we have recorded simultaneously eye-movement data. Thus, this control experiment is evidence that the observed adaptation effects in experiments 1 and 2 are not attributable to eye movements.

\section{Results}

\section{Experiment 1}

In the present study, we used an apparent-rotation sequence as the adapting stimulus that was followed by one of five (static) test stimuli. Figure 1 shows examples of the 
stimuli and a description of the experimental procedure and conditions (see Materials and Methods).

The basic adaptation effect is defined by a decrease in $\mathrm{fMRI}$ signal to the test stimulus, which was identical to the second frame of the apparent-rotation sequence (repeated) in contrast to the test stimulus that is different from the frames of the motion sequence. Critically, a reduction in $\mathrm{fMRI}$ signal to the test stimulus that was rotated to an interpolated (extrapolated) position would indicate that this brain region represents unseen interpolated (extrapolated) rotation views.

The GLM-group analysis (contrast rep < nov) revealed prominent regions bilaterally that demonstrate significantly less $[\mathrm{q}(\mathrm{FDR})=0.05]$ activation for the repeated compared with the novel stimulus. Figure 2 shows the statistical map superimposed on inflated (a) and on flattened (b) surfaces of the reconstructed cortex of both hemispheres of one subject. By their anatomical location and Talairach coordinates (Talairach and Tournoux, 1988), these regions were identified as corresponding to subregions of the occipito-temporal cortex (LOC) (Kourtzi and Kanwisher, 2000; Malach et al., 1995), to subregions of the LOS and the TOS, to subregions of the inferior occipital gyrus (IOG), to subparts of the POS (parieto-occipital sulcus), to the insular cortex (insula), as well as to a region of the right VLPFC (ventrolateral prefrontal cortex).

We investigated the functional response profiles of those ROls that were defined by the basic adaptation effect (rep < nov) in a second stage of analysis. To clarify the amount of adaptation for the unseen interpolated rotation positions with respect to the control conditions (novel, continuous extrapolated, reverse extrapolated), we performed the following contrasts: interpolated versus novel (int $<$ nov), interpolated versus continuous extrapolated (int < conex), and interpolated versus reverse extrapolated (int $<$ revex). The response profiles of the respective regions were deconvolved in time and are shown as event-related time courses (Fig. 2c). GLM parameter estimates averaged across peak points are provided in Additional Figure 1.

We found a significant adaptation effect $(p<0.05)$ for the interpolated condition compared with the novel condition (int < novel) in 13 of 17 predefined ROls (Table 1). More interesting, eight of these regions also demonstrated significant adaptation effects for the interpolated condition in contrast to the extrapolated conditions (int < conex and int < revex): LOC, LOS, LOS/ TOS, and TOS of the left hemisphere as well as LOC anterior, IOG anterior, LOS/TOS, and insula inferior of the right hemisphere (Table 1).

To test whether these eight areas selectively demonstrate adaptation of activation only for the stimuli on the rotation trajectory of the object (repeated, interpolated) in contrast to the stimuli outside the trajectory (continuous extrapolated, reverse extrapolated), we also computed the contrasts continuous extrapolated versus novel (conex $<$ nov) and reverse extrapolated versus novel (revex < nov). Only the subregion of the inferior insula of the right hemisphere demonstrated a significant $(p<0.05)$ adaptation effect for the continuous extrapolated stimulus compared with the novel stimulus (but no adaptation effect for the reverse extrapolated stimulus compared with the novel stimulus) (Additional Table 1). The seven regions that show adaptation of activation for the repeated and interpolated but not for the continuous extrapolated and the reverse extrapolated conditions in contrast to the novel condition are marked with white circles in Figure $2 b$. 

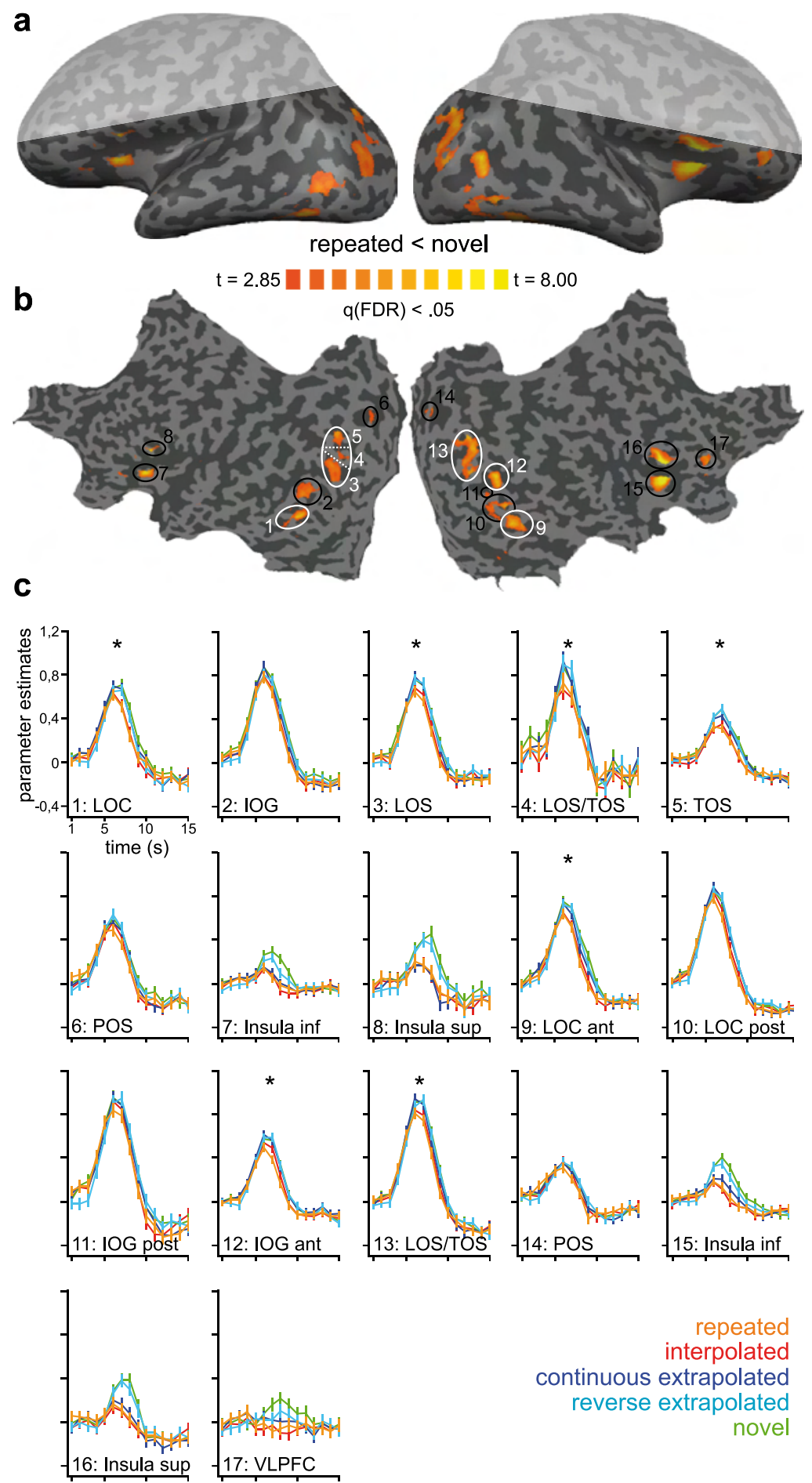

repeated interpolated continuous extrapolated reverse extrapolated novel 
$<<<$

Figure 2. fMRI results for experiment 1.a, Contrast map averaged across subjects showing regions responding significantly less to the repeated stimulus compared with the novel stimulus [contrast rep < nov; basic adaptation effect; $\mathrm{q}(\mathrm{FDR})=0.05$ ] The statistical map is superimposed on inflated cortical surfaces of the left and right hemisphere of one subject. Areas shaded in light gray were not covered because of slice positioning (see Materials and Methods). The sulci are coded in darker gray than the gyri. b, The same statistical map now superimposed on flattened cortical surfaces of the left and right hemisphere of the same subject. Major ROls are circled and numbered. A white circle/number indicates that this region demonstrates significant adaptation effects (ROI-based analysis; $p<0.05)$ for stimuli that lay on the path of motion (repeated, interpolated) in contrast to stimuli that lay off the path of motion (novel, continuous extrapolated, reverse extrapolated). c, Event-related deconvolved BOLD fMRI responses (GLM parameter estimates averaged across trials and subjects for all voxels in each ROI) reported against time for each of the experimental conditions: orange, repeated; red, interpolated; blue, continuous extrapolated; turquoise, reverse extrapolated; green, novel. The ROI numbers correspond to $b$. Time point 1 is trial onset. Error bars indicate SEM. POS, Parietooccipital sulcus; Insula, insular cortex; VLPFC, ventral lateral prefrontal cortex; ant, anterior; post, posterior; inf, inferior; sup, superior.

Together, these results reveal two dominant regions in either hemisphere, one within the ventral visual processing stream (LOC) and one within the dorsal stream (LOS/TOS), and a region in the right IOG that show adaptation of activation for stimuli that lay on the rotation path of the object (interpolated, repeated) compared with stimuli that lay off the path of motion of the object (continuous extrapolated, reverse extrapolated, novel).

\section{Experiment 2}

To confirm our findings and to control for possible alternative explanations, we conducted experiments 2 and 3. Visual stimulation, experimental setting, and scanning procedures were highly similar for experiments 2 and 3. The experiments differed only in the behavioral task subjects had to perform during scanning. Several studies (Eger et al., 2004b; Murray and Wojciulik, 2004; Vuilleumier et al., 2005; Yi and Chun, 2005) have emphasized that attention affects adaptation of activation in the LOC. Because subjects performed an object identification task in experiment 1, we wanted to replicate an object-dependent task in experiment 2 that requires attending to motion features. Thus, we instructed our subjects to indicate whether the test stimulus was rotated in the same direction of motion as the apparent-rotation sequence or in the other direction (DMT). In experiment 3, in contrast, subjects had to perform a demanding task on a randomly generated stream of letters and digits that was presented at the fixation point (ACT). Furthermore, in experiments 2 and 3 (in contrast to experiment 1 ), we slightly varied scanning sequence parameters such that we achieved a total coverage of the brain (see Materials and Methods). In summary, we wanted to replicate our findings of experiment 1 in experiment 2. Furthermore, we controlled for task-specific effects (experiment 1 vs experiment 2) and attention-related effects (experiment 2 vs experiment 3).

To improve the spatial matching between individual subject's brains, we performed a cortex-based intersubject alignment of the anatomical and functional data in experi- 
Table 1. Statistical analysis for experiment 1

\begin{tabular}{|c|c|c|c|c|c|c|c|c|c|c|}
\hline \multirow[b]{2}{*}{ No. } & \multirow[b]{2}{*}{ ROI } & \multicolumn{2}{|c|}{ int $<$ nov } & \multicolumn{2}{|c|}{ int $<$ conex } & \multicolumn{2}{|c|}{ int $<$ revex } & \multicolumn{3}{|c|}{ Talairach } \\
\hline & & $\mathrm{p}$ & $t$ & $\mathrm{p}$ & $t$ & $\mathrm{p}$ & $t$ & $x$ & y & z \\
\hline \multicolumn{11}{|c|}{ Left hemisphere } \\
\hline 1 & LOC & $0.00^{*}$ & 3.56 & $0.01^{*}$ & 2.79 & $0.02^{*}$ & 2.35 & -42 & -56 & -13 \\
\hline 2 & $1 O G$ & $0.02 *$ & 2.49 & 0.13 & 1.51 & 0.12 & 1.58 & -43 & -62 & -3 \\
\hline 3 & LOS & $0.02^{*}$ & 2.52 & $0.01^{*}$ & 2.59 & $0.01^{*}$ & 2.56 & -35 & -76 & 5 \\
\hline 4 & LOS/TOS & $0.05^{*}$ & 2.04 & $0.02 *$ & 2.52 & $0.01^{*}$ & 2.88 & -30 & -84 & 11 \\
\hline 5 & TOS & $0.00^{*}$ & 3.66 & $0.02 *$ & 2.55 & $0.00^{*}$ & 3.64 & -30 & -70 & 17 \\
\hline 6 & POS & 0.01 & 1.66 & 0.25 & 1.17 & 0.08 & 1.75 & -20 & -72 & 19 \\
\hline 7 & Insula, inf & $0.00^{*}$ & 6.48 & 0.30 & 1.03 & $0.00^{*}$ & 3.98 & -28 & 15 & 7 \\
\hline 8 & Insula, sup & $0.00^{*}$ & 4.97 & 0.98 & 0.02 & $0.00^{*}$ & 4.08 & -36 & 11 & 12 \\
\hline \multicolumn{11}{|c|}{ Right hemisphere } \\
\hline 9 & LOC, ant & $0.00^{*}$ & 4.28 & $0.02 *$ & 2.36 & $0.00^{*}$ & 3.76 & 44 & -49 & -12 \\
\hline 10 & LOC, post & 0.06 & 1.92 & $0.03^{*}$ & 2.29 & $0.02^{*}$ & 2.37 & 45 & -61 & -9 \\
\hline 11 & IOG, post & 0.23 & 1.21 & 0.41 & 0.83 & 0.17 & 1.36 & 45 & -68 & -1 \\
\hline 12 & IOG, ant & $0.02 *$ & 2.43 & $0.01^{*}$ & 2.58 & $0.01^{*}$ & 2.80 & 38 & -59 & 10 \\
\hline 13 & LOS/TOS & $0.01^{*}$ & 2.72 & $0.04^{*}$ & 2.09 & $0.03^{*}$ & 2.31 & 38 & -74 & 15 \\
\hline 14 & POS & 0.43 & 0.80 & 0.42 & 0.82 & 0.13 & 1.52 & 25 & -64 & 21 \\
\hline 15 & Insula, inf & $0.00^{*}$ & 7.62 & $0.02^{*}$ & 2.42 & $0.00^{*}$ & 6.12 & 31 & 14 & 7 \\
\hline 16 & Insula, sup & $0.00^{*}$ & 4.06 & 0.22 & 1.23 & $0.00^{*}$ & 4.50 & 41 & 16 & 10 \\
\hline 17 & VLPFC & $0.00^{*}$ & 4.51 & 0.07 & 1.82 & $0.02^{*}$ & 2.37 & 43 & 42 & 6 \\
\hline
\end{tabular}

Data are statistical values for experiment 1 for different pairwise comparisons that were computed on predefined ROIs (ROI defining contrast rep < nov) and Talairach coordinates of the center of the ROI. Talairach conventions are as follows: $x$, left to right; $y$, back to front; $z$, bottom to top. ${ }^{*} p<0.05$. See also Figure 2, Additional Figure 1, and Results, Experiment 1. No., Number; POS, parieto-occipital sulcus; VLPFC, ventral lateral prefrontal cortex.

ents 2 and 3 (see Materials and Methods). It has been shown that a cortical correspondence mapping substantially improves statistical analysis across subjects by reducing anatomical variability (Fischl et al., 1999; Goebel et al., 2006; Meienbrock et al., 2007 van Atteveldt et al., 2004).

We first computed a GLM analysis on the peak points of the averaged time courses (similar as in experiment 1 ) of all subjects using the contrast repeated versus novel (rep $<$ nov). Significantly less activation $[q(F D R)=0.05]$ for the repeated stimulus compared with the novel stimulus (basic adaptation effect) was found again in subregions of the LOS/TOS in both hemispheres and in subregions of the LOC, a region of the posterior fusiform gyrus ( $\mathrm{pFG}$ ), a region of the SPC, a region in the postcentral sulcus, and a region in the SPFC (superior prefrontal cortex) in the right hemisphere. Figure 3 shows the statistical map superimposed on inflated (a) and flattened (b) surfaces of the reconstructed cortex of both hemispheres of one subject. 
In congruence with experiment 1, we also investigated the response profiles of those preselected regions of experiment 2 in an ROI-based analysis. We found adaptation of activation for the unseen interpolated position in contrast to the control conditions (novel, continuous extrapolated, reverse extrapolated) in five of eight ROls: left TOS, right pFG, LOC, LOS/TOS, and SPC (Table 2). Furthermore, none of those areas showed an adaptation effect for the extrapolated conditions compared with the novel condition $(p<0.05)$. Figure $3 c$ shows event-related time courses of the deconvolved MR signal and GLM parameter estimates averaged across peak points.

In summary, the results of experiment 2 nicely confirm the findings from experiment 1: subregions of LOC and LOS/TOS demonstrate a clear adaptation effect for the stimuli on the object's rotation trajectory in contrast to stimuli outside the motion trajectory. Because of the increased brain coverage in experiment 2 compared with experiment 1, an additional region of the SPC was also found to exhibit the specified adaptation effects.

Apart from this highly overlapping cortical network, the involvement of different frontal regions seems to be related to the two different tasks applied in experiments 1 and 2. The OIT task elicited activity in the ventral prefrontal cortex, whereas the DMT activated the dorsal prefrontal cortex. This finding is in line with other investigations demonstrating that the ventral prefrontal cortex is involved in nonspatial tasks, whereas the dorsal prefrontal cortex is more involved in spatial tasks (Courtney et al., 1998; Munk et al., 2002).

\section{Experiment 3}

We conducted a third experiment to test for attention effects. While keeping stimuli the same as in experiments 1 and 2, subjects were instructed to perform an attentiondemanding task on the fixation point in experiment 3 (ACT). To reveal similarities and/

Table 2. Statistical analysis for experiment 2

\begin{tabular}{|c|c|c|c|c|c|c|c|}
\hline \multirow[b]{2}{*}{ No. } & \multirow[b]{2}{*}{ ROI } & \multicolumn{2}{|c|}{ int $<$ nov } & \multicolumn{2}{|c|}{ int $<$ conex } & \multicolumn{2}{|c|}{ int $<$ revex } \\
\hline & & $\mathrm{p}$ & $t$ & $p$ & $t$ & $p$ & $t$ \\
\hline \multicolumn{8}{|c|}{ Left hemisphere } \\
\hline 1 & LOS & $0.04^{*}$ & 2.11 & 0.06 & 1.92 & $0.02^{*}$ & 2.50 \\
\hline 2 & TOS & $0.00^{*}$ & 4.73 & $0.01^{*}$ & 2.81 & $0.00^{*}$ & 4.86 \\
\hline \multicolumn{8}{|c|}{ Right hemisphere } \\
\hline 3 & $\mathrm{pFG}$ & $0.01^{*}$ & 2.96 & $0.03^{*}$ & 2.17 & $0.01^{*}$ & 2.94 \\
\hline 4 & LOC & $0.01^{*}$ & 2.86 & $0.01^{*}$ & 3.14 & $0.01 *$ & 3.18 \\
\hline 5 & LOS/TOS & $0.00^{*}$ & 4.63 & $0.01^{*}$ & 2.88 & $0.00^{*}$ & 4.35 \\
\hline 6 & SPC & $0.01^{*}$ & 3.20 & $0.01^{*}$ & 2.56 & $0.04^{*}$ & 2.12 \\
\hline 7 & postCS & $0.01^{*}$ & 3.11 & $0.01^{*}$ & 2.75 & 0.12 & 1.56 \\
\hline 8 & SPFC & 0.19 & 1.32 & 0.39 & 0.87 & 0.03 & \\
\hline
\end{tabular}

Data are statistical values for experiment 2 for different pairwise comparisons that were computed on predefined ROIs (ROI defining contrast rep $<$ nov). ${ }^{*} p<0.05$. See also Figure 3 and Results, Experiment 2. postCS, postcentral sulcus; SPFC, superior prefrontal cortex. 

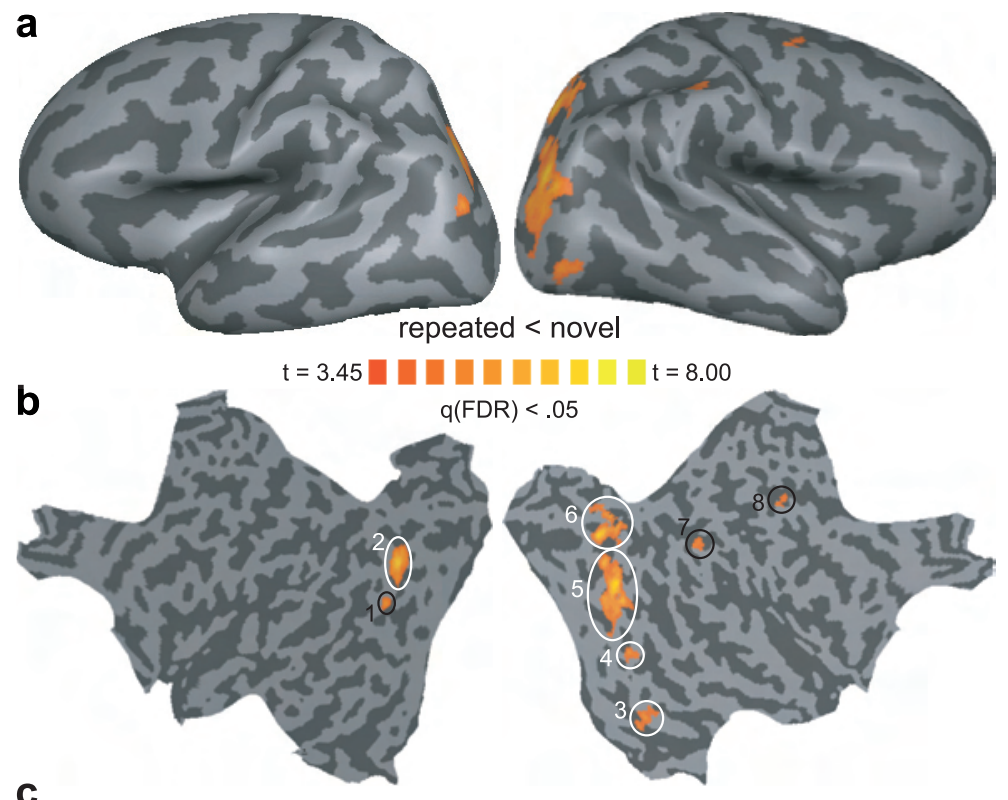

C
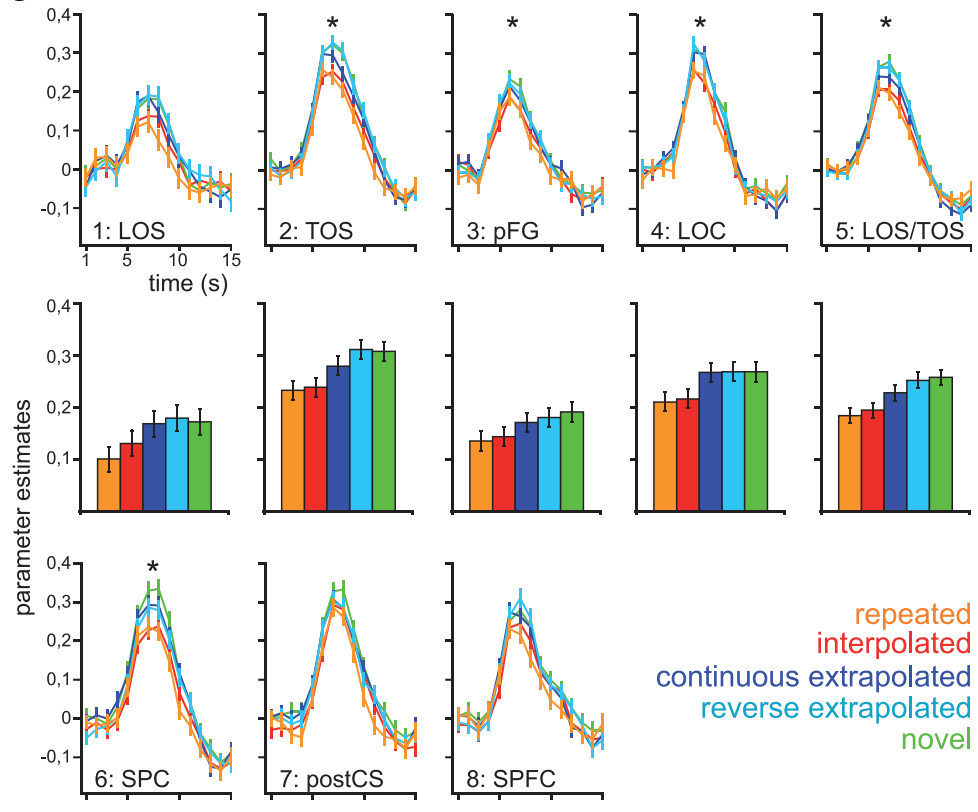

repeated interpolated continuous extrapolated reverse extrapolated novel
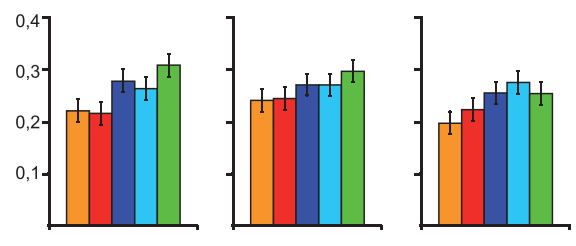
$<<<$

Figure 3. fMRI results for experiment 2. a, Contrast map averaged across subjects (using the cortex-based intersubject alignment approach; see Materials and Methods) showing regions responding significantly less to the repeated stimulus compared with the novel stimulus [contrast rep $<$ nov; basic adaptation effect; $q(F D R)=0.05]$. The statistical map is superimposed on inflated cortical surfaces of the left and right hemisphere of one subject. The sulci are coded in darker gray than the gyri. b, The same statistical map now superimposed on flattened cortical surfaces of the left and right hemisphere of the same subject. Major ROls are circled and numbered. A white circle/number indicates that this region demonstrates significant adaptation effects (ROI-based analysis; $p<0.05$ ) for stimuli that lay on the path of motion (repeated, interpolated) in contrast to stimuli that lay off the path of motion (novel, continuous extrapolated, reverse extrapolated). c, Event-related deconvolved BOLD fMRI responses (GLM parameter estimates, averaged across trials and subjects for all voxels in each ROI) reported against time for each of the experimental conditions and BOLD fMRI responses (GLM parameter estimates averaged across peak points (5-7 s after trial onset) and trials and subjects for all voxels in each ROI) for each of the experimental conditions: orange, repeated; red, interpolated; blue, continuous extrapolated; turquoise, reverse extrapolated; green, novel. The ROI numbers correspond to $b$. Time point 1 is trial onset. Error bars indicate SEM. postCS, Postcentral sulcus; SPFC, superior prefrontal cortex.

or differences caused by the different tasks, we wanted to directly compare the results of experiment 2 (DMT) with results obtained in experiment 3 (ACT). We therefore matched the number of subjects of both experiments by randomly selecting six subjects of the total population of nine for experiment 2. We then applied the same statistical analysis as in experiment 1 for the subpopulation of experiment 2 and for experiment 3 including cortex-based intersubject alignment and GLM group analyses on the peak points of the averaged time course (contrast repeated vs novel, rep < nov).

Figure 4 shows the statistical maps for both tasks (orange, DMT; purple, ACT) overlaid on inflated cortical surfaces of one subject. The functional activation maps are defined by the contrast repeated versus novel (rep $<$ nov) and are shown at two different thresholds, $q(F D R)=0.05$ (Fig. 4a) and $p<0.05$ (uncorrected) (Fig. 4b). The statistical map for the subpopulation of experiment 2 reveals the same pattern of activity as for the total population of experiment 2; however, the statistical map for experiment 3 looks quite different: on a high threshold $[q(F D R)=0.05]$, only a small region in the right intraparietal sulcus demonstrates significantly less activity for the repeated compared with the novel condition (Fig. 4a). Lowering the threshold to $p<0.05$ (uncorrected) reveals a patchy pattern of activity in mainly occipital, parietal, and temporal regions (Fig. 4a). On the lower threshold, both contrast maps overlap at four distinct regions: a region in the left LOS (1), a region in the left junction of the intraparietal and the transverse occipital sulcus (2), a region in the right transverse occipital sulcus (3), and a region in the SPC (4).

We also investigated the response profiles of those overlapping regions that were defined by the more liberal threshold by conducting ROI-based analyses for each experiment, respectively (DMT, ACT). Figure 4c displays event-related time courses of the deconvolved MR signal of the overlapping regions for each experiment (orange frame, DMT; purple frame, ACT). GLM parameter estimates averaged across peak points are provided in Additional Figure 2. For the DMT experiment, three of four ROls demon- 
a

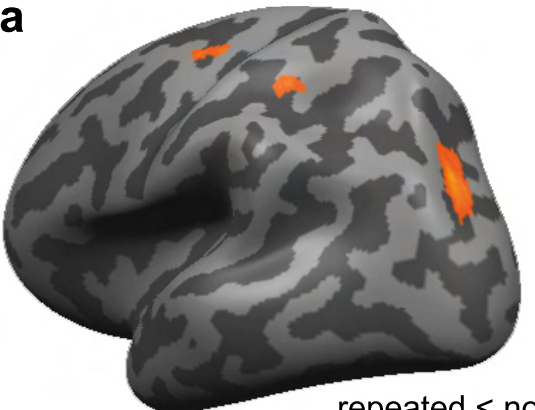

repeated < novel; $\mathrm{q}(\mathrm{FDR})<.05$

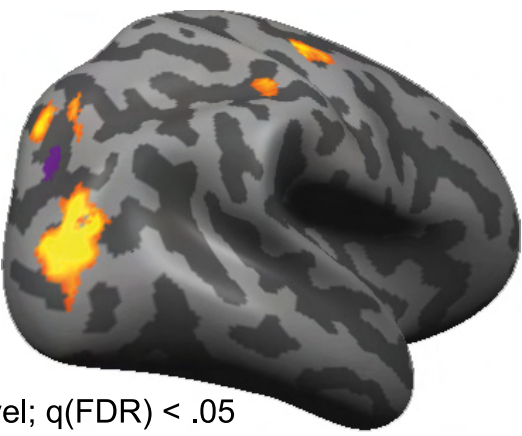

Center task (ACT) $\mathrm{t}=4.8 \square \square \square=8.0$

$$
\mathrm{t}=3.2 \square \mathrm{t}=5.0
$$

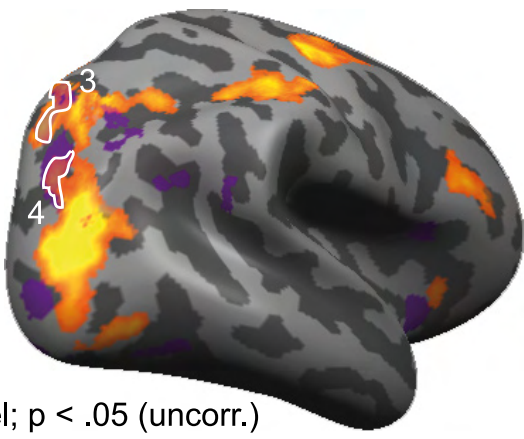

Object task (DMT)

Center task (ACT)

b

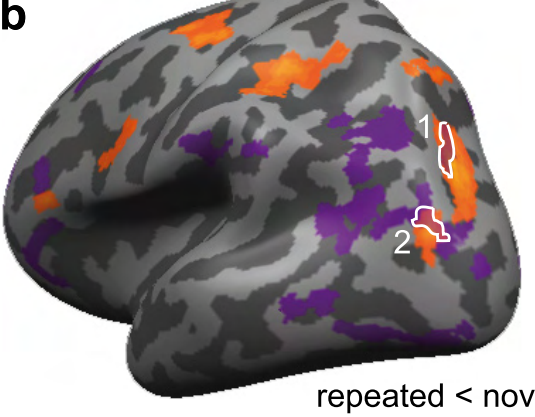

repeated < novel; $p<.05$ (uncorr.)

$\mathrm{t}=2.0$ $\mathrm{t}=2.0 \square \square \square=8.0$

C

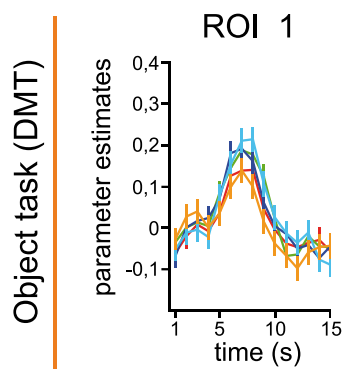

ROI 2
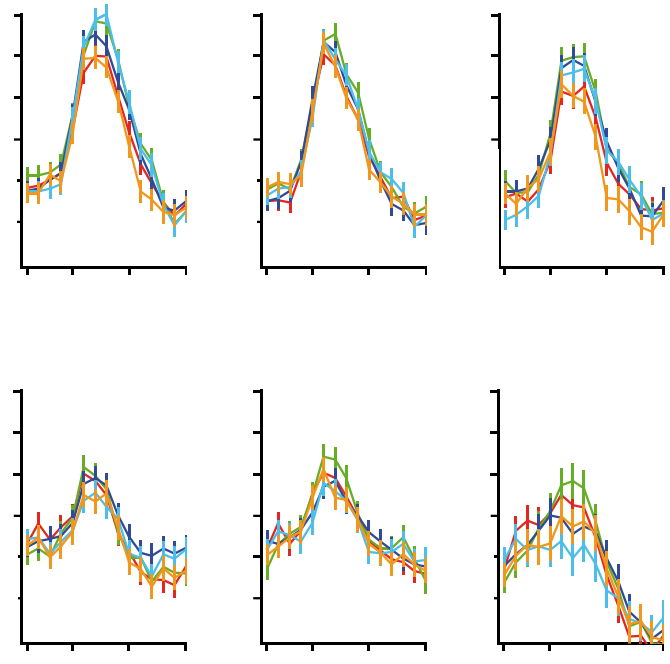

repeated interpolated

cont. extrapolated

rev. extrapolated

novel 
$<<<$

Figure 4. Comparison of fMRI results for experiments 2 and 3. a, Contrast maps averaged across subjects (using the cortex-based intersubject alignment approach; see Materials and Methods) for the object task (DMT; orange) and the center task (ACT; purple) showing regions responding significantly less $[q(F D R)=0.05]$ to the repeated stimulus compared with the novel stimulus (contrast rep $<$ nov). The statistical maps are superimposed on inflated cortical surfaces of the left and right hemisphere of one subject. The sulci are coded in darker gray than the gyri. b, The same contrast maps as in a on a lower threshold [p $<0.05$, uncorrected (uncorr.)]. Overlapping regions are marked with white lines and are numbered. c, Event-related deconvolved BOLD fMRI responses (GLM parameter estimates, averaged across trials and subjects for all voxels in each ROI) reported against time for each of the experimental conditions: orange, repeated; red, interpolated; blue, continuous extrapolated (cont. extrapolated); turquoise, reverse extrapolated (rev. extrapolated); green, novel. Always, two MR-signal time courses refer to the same ROI in the two different experiments. Time point 1 is trial onset. Error bars indicate SEM.

strated a significant adaptation effect $(p<0.05)$ for the unseen interpolated stimulus in contrast to the novel stimulus (Table 3). For the ACT experiment, however, none of the regions exhibited an adaptation effect for the unseen interpolated stimulus in contrast to the novel stimulus (Table 3 ). Three of four ROls demonstrated a significant adaptation effect $(p<0.05)$ for the interpolated stimulus in contrast to the continuous extrapolated stimulus in the DMT experiment, whereas none of the regions showed this effect for the ACT experiment (Table 3). Furthermore, for the DMT experiment, two regions exhibited adaptation of activation for the interpolated stimulus in contrast to the reverse extrapolated stimulus, but no region did so in the ACT experiment (Table 3).

These results suggest that attention plays a major role for the integration of an apparent-rotation sequence. If attention is drawn away from the rotating objects to a demanding task on the fixation point, the cortical network normally involved in representing interpolated rotation positions does not show this specificity any longer.

\section{Experiment 4}

Experiment 4 was designed to confirm that the observed adaptation effects (experiments 1 and 2) were actually based on the perception of apparent motion and not simply because of conventional adaptation effects based on image similarity or overlapping tuning functions for specific rotation angles. We therefore manipulated the original visual presentation: instead of presenting an apparent-rotation sequence as the adapting stimulus, we presented two double stimuli. These stimuli were created by superimposing the original first frame on top of the original second frame of the apparent-rotation sequence and vice versa (Fig. 1a, experiment 4) (see Materials and Methods for a more detailed description of the procedure). The presentation of the two double stimuli no longer elicited the perception of apparent motion, but subjects reported to see two flickering objects.

We again computed a multisubject GLM analysis on the peak points of the averaged time courses (as in experiments 1-3) using the contrast repeated versus novel (rep < nov). An adaptation effect ( $p<0.05$, corrected) for the repeated stimulus compared with the novel stimulus (basic adaptation effect) was found in a region of the right LOC and in regions of the SPC. Figure 5a displays the statistical map superimposed on 
Table 3. Statistical analysis for experiment 3

\begin{tabular}{|c|c|c|c|c|c|c|c|}
\hline \multirow[b]{2}{*}{ No. } & \multirow[b]{2}{*}{ Task } & \multicolumn{2}{|c|}{ int $<$ nov } & \multicolumn{2}{|c|}{ int $<$ conex } & \multicolumn{2}{|c|}{ int $<$ revex } \\
\hline & & $\mathrm{p}$ & $t$ & $\mathrm{p}$ & $t$ & $\mathrm{p}$ & $t$ \\
\hline \multicolumn{8}{|c|}{ Left hemisphere } \\
\hline \multirow[t]{2}{*}{1} & DMT & 0.12 & 1.67 & $0.05^{*}$ & 1.98 & $0.01 *$ & 2.70 \\
\hline & $\mathrm{ACT}$ & 0.62 & 0.49 & 0.86 & -0.18 & 0.27 & -1.10 \\
\hline \multirow[t]{2}{*}{2} & DMT & $0.01^{*}$ & 3.26 & $0.02 *$ & 2.44 & $0.00^{*}$ & 4.02 \\
\hline & $\mathrm{ACT}$ & 0.51 & 0.65 & 0.94 & 0.08 & 0.09 & -1.74 \\
\hline \multicolumn{8}{|c|}{ Right hemisphere } \\
\hline \multirow[t]{2}{*}{3} & DMT & $0.01^{*}$ & 2.71 & 0.15 & 0.11 & 0.11 & 1.63 \\
\hline & $\mathrm{ACT}$ & 0.06 & 1.94 & 0.40 & -0.84 & 0.30 & -1.04 \\
\hline \multirow[t]{2}{*}{4} & DMT & $0.01^{*}$ & 3.25 & $0.01^{*}$ & 2.60 & 0.07 & 1.86 \\
\hline & $\mathrm{ACT}$ & 0.26 & 1.14 & 0.13 & -1.51 & 0.01 & -2.96 \\
\hline
\end{tabular}

Data are statistical values for a comparison between experiment 2 (DMT) and experiment 3 (ACT) for different pairwise comparisons that were computed on predefined ROls (overlapping regions as shown in Fig. 4). ${ }^{*} p<0.05$. See also Additional Figure 2 and Results, Experiment 3.

a sagittal and a coronal slice of one subject. As in experiments 1-3, we performed additional analyses on the so-defined ROIs (Additional Table 2). None of the five different pairwise comparisons (int $<$ nov, int $<$ conex, int $<$ revex, conex $<$ nov, revex $<$ nov) was significant for the region in the right LOC $(p>0.05)$. The two ROls in the parietal cortex revealed a significant adaptation effect $(p<0.05)$ for the interpolated stimulus in contrast to the novel stimulus and for the continuous extrapolated stimulus in contrast to the novel stimulus. The region in the right parietal cortex additionally showed a significant adaptation effect $(p<0.05)$ also for the reverse extrapolated stimulus in contrast to the novel stimulus. However, most interesting, the ROls in the parietal cortex also did not show an adaptation effect for the interpolated stimulus in contrast to either the continuous or the reverse extrapolated stimulus ( $p>0.05)$. Figure $5 b$ shows eventrelated time courses of the deconvolved MR signal and GLM parameter estimates averaged across peak points for the three ROIs.

Thus, the elimination of the motion perception had a major impact on the previously described network. This findings support our conclusion from experiments 1 and 2 that the observed adaptation effects to the interpolated stimulus were actually based on the perceived apparent rotation.

\section{Discussion}

The goal of our study was to investigate the effect of motion on the representation of 3D objects. In apparent rotation, different views of an object are linked by (apparent) motion, thereby creating the illusion of a smooth rotational object motion. In the present fMRI study, we wanted to localize cortical regions that show motion-specific, viewinvariant representations of rotating $3 \mathrm{D}$ objects. We found a cortical network com- 

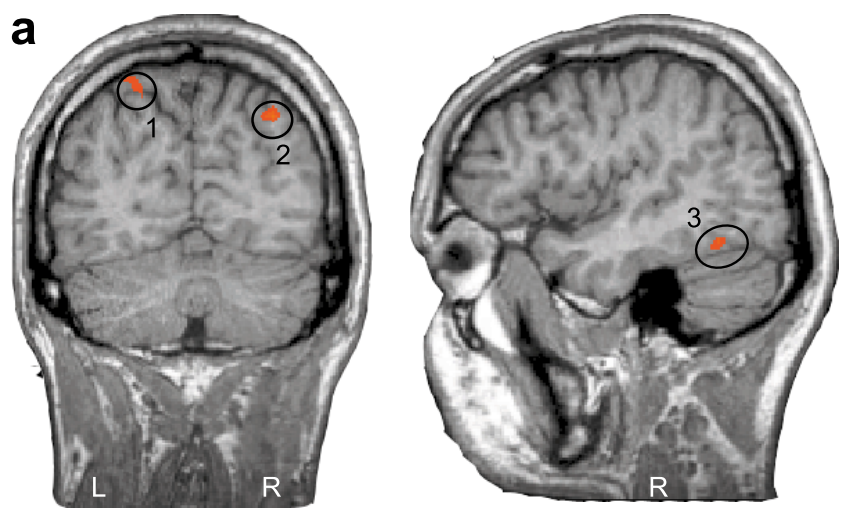

repeated < novel

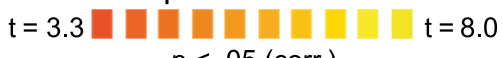

$\mathrm{p}<.05$ (corr.)

b

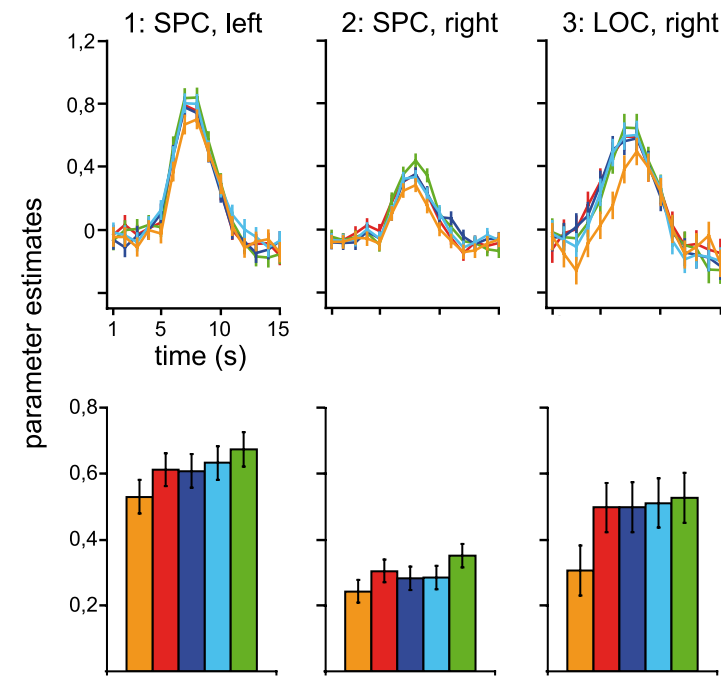

repeated interpolated cont. extrap. rev. extrap. novel

Figure 5. fMRI results for experiment 4. a, Contrast map averaged across subjects showing regions responding significantly less to the repeated stimulus compared with the novel stimulus [contrast rep $<$ nov; basic adaptation effect; $p<0.05$, corrected (corr.)]. The statistical map is superimposed on a sagittal and a coronal slice of one subject. L, Left; R, right. b, Event-related deconvolved BOLD fMRI responses (GLM parameter estimates, averaged across trials and subjects for all voxels in each ROI) reported against time for each of the experimental conditions and BOLD FMRI responses (GLM parameter estimates averaged across peak points (5-7 s after trial onset) and trials and subjects for all voxels in each ROI) for each of the experimental conditions: orange, repeated; red, interpolated; blue, continuous extrapolated (cont. extrap.); turquoise, reverse extrapolated (rev. extrap.); green, novel. The ROI numbers correspond to a. Time point 1 is trial onset. Error bars indicate SEM. 
prising areas of the ventral and dorsal processing streams (Ungerleider and Mishkin, 1982) of both hemispheres that showed adaptation to stimuli that lay on the rotation trajectory of the object, but no adaptation to stimuli that lay off that path. In particular, regions in the LOC and the TOS/LOS in both hemispheres and regions of the right IOG (rIOG) and of the SPC of only the right hemisphere showed the specified adaptation profiles. Thus, these areas seem to encode those views of a rotating 3D object that are linked by motion. Our findings thus support psychophysical evidence (Kourtzi and Shiffrar, 1997; 1999; McNamara et al., 2006) showing that linking different views of a 3D object through motion does not lead to a complete view-invariant representation of the rotating object but to a representation that is restricted to the rotation trajectory. A highly similar network has been found investigating the phenomenon of structure from motion (SFM). In SFM, moving dots evoke the perception of a 3D object in the absence of any other visual object cue (Treue et al., 1991; Wallach and O'Connell, 1953). The cortical network representing SFM comprises areas in the dorsal visual pathway, namely the human motion complex (hMT+/V5), the intraparietal sulcus, and other parts of the parietal cortex and areas in the ventral visual pathway, predominately the LOC (Kriegeskorte et al., 2003). Thus, both motion- and object-selective regions are involved in the representation of SFM. SFM and apparent rotation are highly related because, in both cases, the perception of an object is generated through (apparent) motion. Thus, it is not surprising that a similar cortical network underlies both phenomena. We think that the interaction of dorsal and ventral areas in the visual processing hierarchy forms the basis for the perception of apparent rotation. But, in contrast to SFM, in which motion per se leads to the perception of an object, in apparent rotation the influence of motion is more subtle. In apparent rotation, motion processing influences one feature of a rotating object, namely its viewpoint.

\section{Attention- and task-related effects}

The focus of attention has an effect on the cortical network involved in representing the interpolated rotation positions. We observed several changes within the reported network when the focus of attention is drawn away from the objects (experiment 3 ). First, the level of cortical activation within the reported network is strongly reduced. Moreover, the specific effect of adaptation toward the interpolated position vanishes (i.e., above the overall reduced power, there is a specific effect on the differential activation pattern). Although we were still able to find areas that showed the basic adaptation effect, these areas no longer displayed adaptation to the interpolated position. This is in line with previous studies showing that it is essential for adaptation effects to occur that attention is directed to the objects under study (Eger et al., 2004b; Murray and Wojciulik, 2004; Vuilleumier et al., 2005; Yi and Chun, 2005). If in our study the objects are not attended, the neuronal assemblies might not involve in the representation of the interpolated positions.

Despite the global influence attention has on adaptation effects, we are confident that the differential adaptation profile for the experimental conditions is not attributable to attention effects. First, observers were not able to anticipate the condition of the upcoming trial, because our event-related design used randomized presentation of trials from all conditions. Furthermore, we used two different object-related tasks leading to essentially similar results. This observation is in line with a study by Murray 
and Wojciulik (2004) demonstrating that, as long as attention is focused on the objects, the differential adaptation profile of a cortical region representing the attended objects is not affected by the type of task performed.

\section{Motion specificity}

In experiment 4, we eliminated the motion illusion, and this manipulation had two effects on the previously described cortical network representing the interpolated stimulus. First, only subparts of the original network (LOC and SPC) now still displayed the basic adaptation effect (adaptation of activation for the repeated stimulus in contrast to the novel stimulus). Second and most interesting, those regions no longer displayed the specific adaptation effects to the interpolated stimulus in contrast to the extrapolated stimuli. LOC only showed adaptation to the repeated stimulus and no adaptation to interpolated or extrapolated stimuli, whereas regions in the SPC displayed a pronounced adaptation effect to the repeated stimulus and also some adaptation for the interpolated as well as the extrapolated stimuli (all in contrast to the novel stimulus). We interpret these adaptation profiles as demonstrations of (static) viewpoint effects on the representations of objects in temporal and parietal regions. This result is in agreement with several other studies investigating the effect of viewpoint on static object representation (Gauthier et al., 2002; Grill-Spector et al., 1999; James et al., 2002; Vuilleumier et al., 2002). Most of the studies found viewpoint-independent representations for rotation angles up to $60 \mathrm{deg}$ in ventral visual areas such as the LOC (GrillSpector et al., 1999; James et al., 2002; Vuilleumier et al., 2002) (but see Gauthier et al., 2002). In contrast, object representation in the parietal cortex has been shown to be viewpoint dependent (James et al., 2002; Vuilleumier et al., 2002).

In the present study, we evaluated the effects of viewpoint on the representation of 3D objects both during static presentation and during motion presentation. Thus, we are able to extend the previous findings by stating that the same regions showing static viewpoint effects and additional regions in the dorsal pathway (LOS/TOS, rIOG) display viewpoint effects that are motion specific (i.e., as soon as objects are seen in motion, those regions additionally code interpolated rotation positions). We did not observe a complete viewpoint-invariant representation of the object, but the invariance was restricted to views of the object that lay on its rotation trajectory. Thus, we conclude that a cortical network of regions in the temporal and parietal cortices displays viewpoint effects that are motion specific (Stone, 1999).

In conclusion, our study provides additional insights into the cortical representation of 3D objects rotating in depth. More precisely, our four fMRI-adaptation experiments show that a cortical network of areas in the ventral and dorsal stream of the visual processing hierarchy subserves the on-line integration of motion-specific and view-specific features of rotating objects. Furthermore, our study expands the fMRIadaptation method to the study of dynamical stimuli. To our knowledge, this is the first study combining a motion sequence and a static stimulus in a cross-adaptation approach to assess functional properties of brain regions processing dynamic stimuli. Adaptation experiments so far used either two motion sequences (Huettel et al., 2004; Krekelberg et al., 2005) or two static images (Grill-Spector et al., 1999; James et al., 2002; Kourtzi and Kanwisher, 2000; 2001; Kourtzi et al., 2003a, b; Valyear et al., 2006 Vuilleumier et al., 2002). 

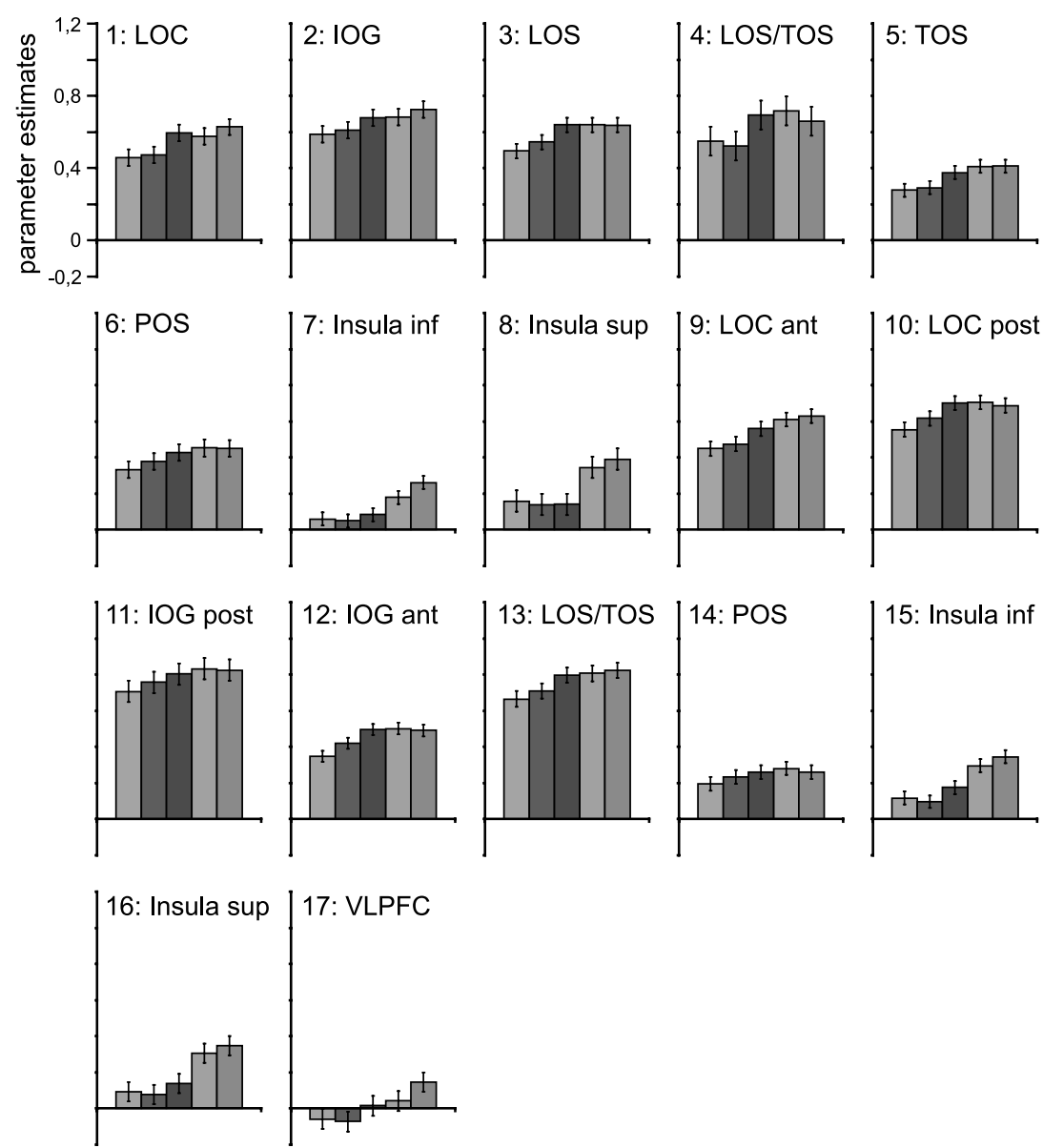

\section{repeated interpolated cont. extrapolated rev. extrapolated novel}

Additional Figure 1. fMRI results for Experiment 1 - bar plots. BOLD fMRI responses (= GLM parameter estimates, averaged across peak points $(5,6$, and $7 \mathrm{~s}$ after trial onset) and trials and subjects for all voxels in each ROI) for each of the experimental conditions: repeated [orange], interpolated [red], continuous extrapolated [blue], reverse extrapolated [turquoise], and novel [green]. The ROI-numbers correspond to Figure 2b. Error bars correspond to standard errors of the mean. Abbreviations: LOC, lateral occipital complex; IOG, inferior occipital gyrus; LOS, lateral occipital sulcus; TOS, transverse occipital sulcus; POS, parieto-occipital sulcus; Insula, insular cortex; VLPFC, ventral lateral prefrontal cortex; ant/post/inf/sup refer to anterior/posterior/inferior/superior. 


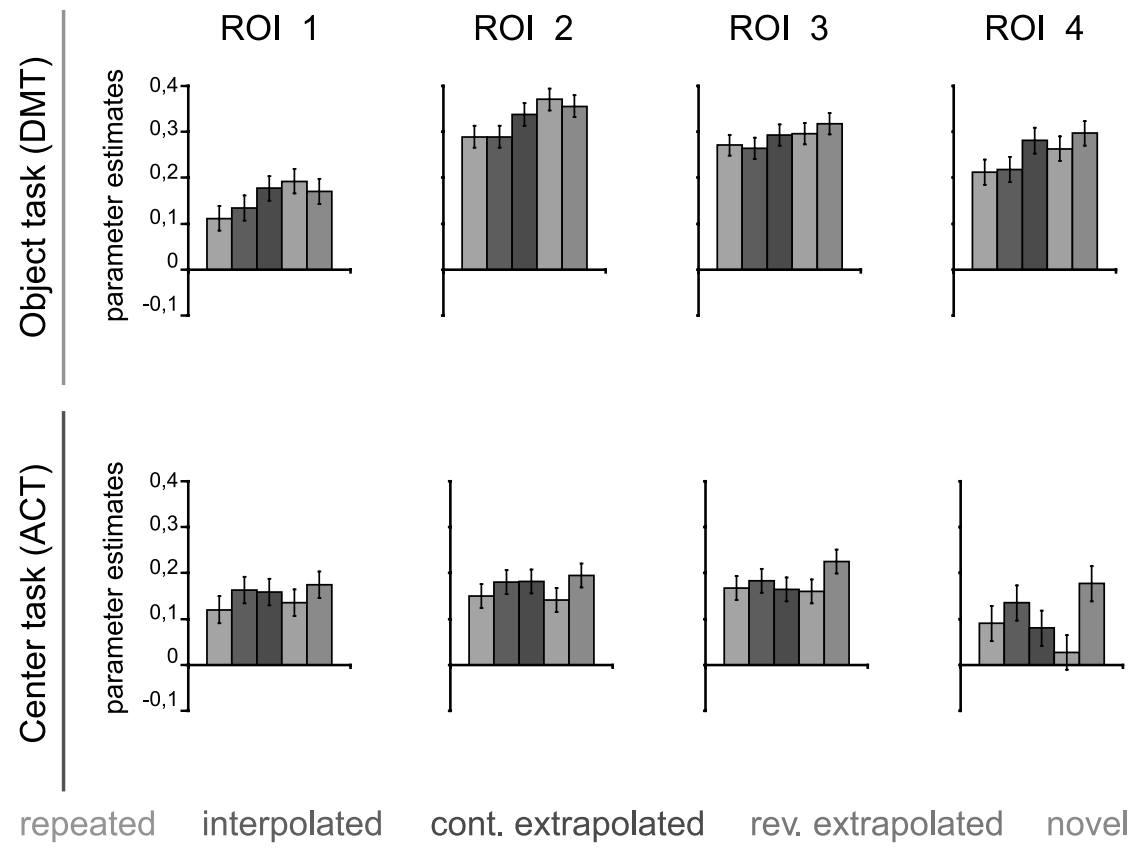

Additional Figure 2. Comparison of fMRI results for Experiments 2 and 3 - bar plots. BOLD fMRI responses (= GLM parameter estimates, averaged across peak points $(5,6$, and $7 \mathrm{~s}$ after trial onset) and trials and subjects for all voxels in each ROI) for each of the experimental conditions: repeated [orange], interpolated [red], continuous extrapolated [blue], reverse extrapolated [turquoise], and novel [green]. The ROI-numbers are corresponding to Figure 4b. Error bars correspond to standard errors of the mean. Always two bar plots refer to the same ROI in the two different experiments. 

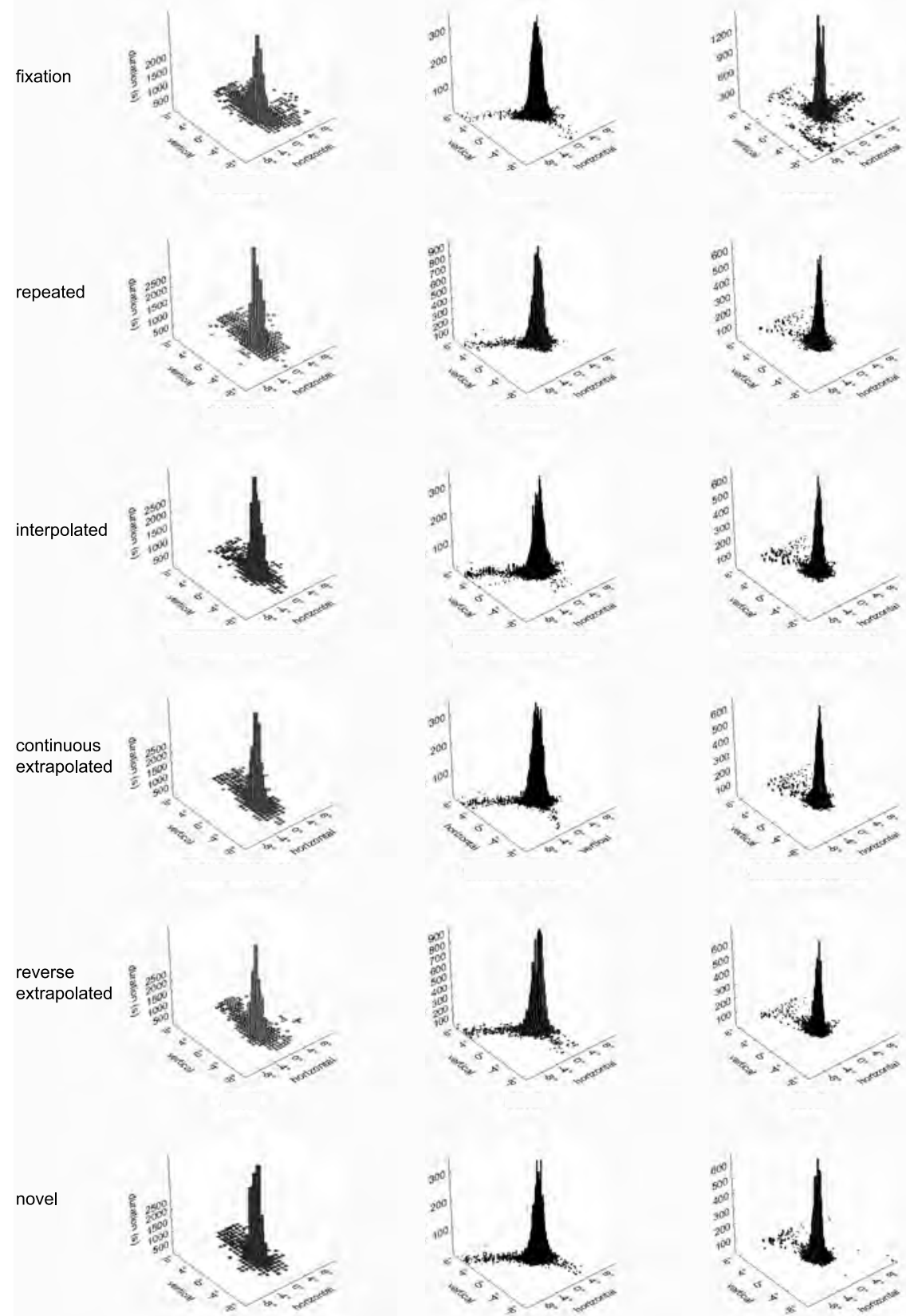

Subject 1

Subject 2

Subject 3 
$<<<$

Additional Figure 3. 2D fixation-density plots Two-dimensional fixation-density plots are shown for each participant and each condition. $X$ - and Y-axes refer to horizontal and vertical eye position in degrees of visual angle (0/0 being the center of the screen). The Z-axis displays the amount of time spent in this position in seconds.

a

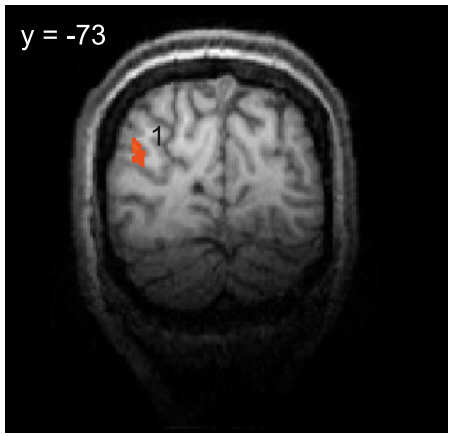

rep. < novel $\cap$ inter. < (conex and revex), balanced

$\mathrm{t}=2.0 \square \square \mathrm{t}=8.0$

$p<.045$ (uncorr.)

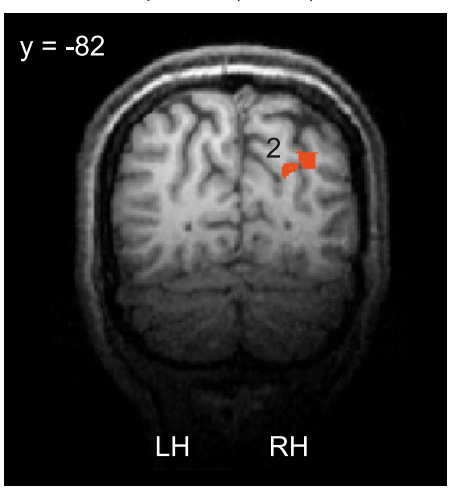

b

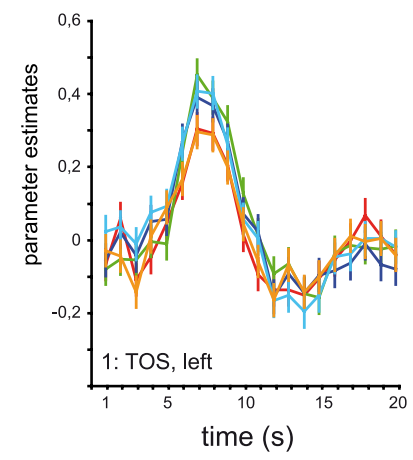

repeated interpolated continuous extrapolated reverse extrapolated novel

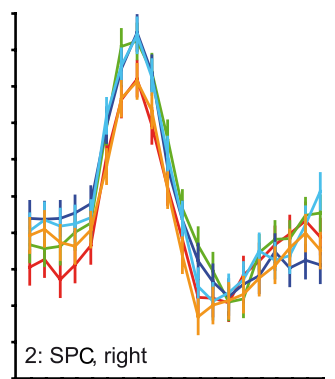

Additional Figure 4. fMRI results for the three subjects of the eye tracking experiment. a, Contrast map averaged across subjects showing two regions responding significantly less to the repeated stimulus in comparison to the novel stimulus (contrast rep $<$ nov) and at the same time (conjunction analysis) responding less to the interpolated stimulus in comparison to the extrapolated stimuli (balanced contrast int $<$ conex and revex) at $p<.045$, uncorrected). The statistical map is superimposed on two coronal slices ( $y=-73$ and $y=-82$ ) of one subject to demonstrate the activated regions in parietal cortex. $b$, Event-related deconvolved BOLD FMRI responses (= parameter estimates, averaged across trials and subjects for all voxels in each ROI) reported against time for each of the experimental conditions: repeated [orange], interpolated [red], continuous extrapolated [blue], reverse extrapolated [turquoise], and novel [green]. The ROI-numbers are corresponding to a. Time point 1 = trial onset. Error bars correspond to standard errors of the mean. Abbreviations: TOS, transverse occipital sulcus; SPC, superior parietal cortex. 
Additional Table 2. Additional statistical analysis for experiment 1

\begin{tabular}{llllll}
\hline & & \multicolumn{2}{l}{ conex < nov } & \multicolumn{2}{l}{ revex < conex } \\
\hline No. & ROI & $p$ & $\dagger$ & $p$ & $\dagger$ \\
\hline Left hemisphere & & & & \\
1 & LOC & 0.45 & 0.76 & 0.23 & 1.12 \\
2 & IOG & 0.33 & 0.97 & 0.37 & 0.90 \\
3 & LOS & 0.93 & -0.06 & 0.98 & -0.03 \\
4 & LOS/TOS & 0.64 & -0.47 & 0.41 & -0.83 \\
5 & TOS & 0.27 & 1.11 & 0.99 & 0.02 \\
6 & POS & 0.62 & 0.50 & 0.94 & -0.08 \\
7 & Insula, inf & $0.00^{*}$ & 5.47 & $0.02 *$ & 2.51 \\
8 & Insula, sup & $0.00 *$ & 4.96 & 0.37 & 0.90 \\
Right hemisphere & & & & \\
9 & LOC, ant & 0.06 & 1.92 & 0.61 & 0.52 \\
10 & LOC, post & 0.78 & -0.37 & 0.66 & -0.45 \\
11 & IOG, post & 0.70 & 0.39 & 0.85 & -0.14 \\
12 & IOG, ant & 0.88 & -0.15 & 0.72 & -0.36 \\
13 & LOS/TOS & 0.53 & 0.62 & 0.69 & 0.40 \\
14 & POS & 0.99 & -0.01 & 0.48 & -0.71 \\
15 & Insula, inf & $0.00 *$ & 5.22 & 0.13 & 1.52 \\
16 & Insula, sup & $0.00 *$ & 4.06 & 0.44 & 0.78 \\
17 & VLPFC & $0.01 *$ & 2.70 & $0.03 *$ & 2.14 \\
\hline Addition & & & & \\
\hline
\end{tabular}

Additional statistical values for Experiment 1 for different pairwise comparisons that were computed on pre-defined ROIs (ROI defining contrast rep < nov) and Talairach coordinates of the center of the ROI. Talairach conventions: $x$ - left to right, $y$ - back to front, $z$ - bottom to top, ${ }^{*}$ $p<.05$. See also Fig. 2 and the results section Experiment 1.

Additional Table 3. Statistical analysis for Experiment 4

\begin{tabular}{|c|c|c|c|c|c|c|c|c|c|c|c|}
\hline \multirow[b]{2}{*}{ No. } & \multirow[b]{2}{*}{ ROI } & \multicolumn{2}{|c|}{ int $<$ nov } & \multicolumn{2}{|c|}{ int $<$ conex } & \multicolumn{2}{|c|}{ int $<$ revex } & \multicolumn{2}{|c|}{ conex $<$ nov } & \multicolumn{2}{|c|}{ revex $<$ nov } \\
\hline & & $p$ & $t$ & $p$ & $t$ & $p$ & $t$ & $\mathrm{p}$ & $t$ & $p$ & $t$ \\
\hline \multicolumn{12}{|c|}{ Left hemisphere } \\
\hline 1 & SPC & $0.05^{*}$ & 1.96 & 0.90 & -0.12 & 0.51 & 0.65 & $0.04^{*}$ & 2.08 & 0.19 & 1.31 \\
\hline \multicolumn{12}{|c|}{ Right hemisphere } \\
\hline 2 & SPC & $0.03^{*}$ & 2.19 & 0.30 & -1.04 & 0.35 & -0.93 & $0.00^{*}$ & 3.23 & 0.00 & 3.13 \\
\hline 3 & LOC & 0.54 & 0.62 & 1.0 & 0.02 & 0.78 & 0.28 & 0.55 & 0.59 & 0.74 & 0.33 \\
\hline
\end{tabular}

Statistical values for Experiment 4 for different pairwise comparisons that were computed on pre-defined ROls (ROI defining contrast rep $<$ nov). ${ }^{*} p<.05$. See also Fig. 5 and the results section Experiment 4. 


\title{
Separate cortical stages in amodal completion
}

\author{
Revealed by functional magnetic \\ resonance adaptation
}

Objects in our environment are often partly occluded, yet we effortlessly perceive them as whole and complete. This phenomenon is called visual amodal completion. Psychophysical investigations suggest that the process of completion starts from a representation of the (visible) physical features of the stimulus and ends with a completed representation of the stimulus. The goal of our study was to investigate both stages of the completion process by localizing both brain regions involved in processing the physical features of the stimulus as well as brain regions representing the completed stimulus. Using $\mathrm{fMRI}$ adaptation we reveal clearly distinct regions in the visual cortex of humans involved in processing of amodal completion: early visual cortex presumably $\mathrm{V} 1$ - processes the local contour information of the stimulus whereas regions in the inferior temporal cortex represent the completed shape. Furthermore, our data suggest that at the level of inferior temporal cortex information regarding the original local contour information is not preserved but replaced by the representation of the amodally completed percept. These findings provide neuroimaging evidence for a multiple step theory of amodal completion and further insights into the neuronal correlates of visual perception.

Weigelt, S., Singer, W., and Muckli, L. (2007). Separate cortical stages in amodal completion revealed by functional magnetic resonance adaptation. BMC Neuroscience, 8:70. 


\section{Background}

In our natural environment we often encounter partly occluded objects. However, what we often perceive in a swift and effortless manner is a whole and complete object (see Figure 1 for an exemplar stimulus). Michotte et al. (1964) called this phenomenon visual amodal completion. Systematic psychophysical investigations suggest a sequential process of completion including several stages starting with an initial representation of the (visible) physical stimulus features and ending with a completed representation of the stimulus (Rauschenberger and Yantis, 2001; Sekuler and Palmer, 1992). So far, neuroimaging studies of amodal completion concentrated on the latter stage in the completion process, demonstrating that higher visual areas encode completed objects (Kourtzi and Kanwisher, 2001; Lerner et al. 2002; Murray et al, 2004). The goal of our study was to investigate both stages of the completion process by localizing both brain regions involved in processing the physical features of the stimulus as well as brain regions representing the completed stimulus.

To identify brain regions representing different stages of the process of amodal completion we employed a functional magnetic resonance (fMRI) adaptation paradigm (Grill-Spector and Malach, 2001; Grill-Spector et al., 2006; Krekelberg et al., 2006). In a typical event-related fMRI adaptation paradigm two stimuli are presented sequentially (Kourtzi and Kanwisher, 2000;2001). In case the two stimuli are functionally similar thus activating the same or highly overlapping neuronal subpopulations this causes a decrease in the $\mathrm{FMRI}$ signal to the second stimulus (adaptation). No such decrease in fMRI signal occurs if the two stimuli are functionally dissimilar activating different subpopulations of neurons (no adaptation). Thus, using fMRI adaptation, the functional characteristics of brain regions can be assessed at the level of neuronal subpopulations, even if those are spatially overlapping and not separable with conventional fMRI. Accordingly, fMRI adaptation refers to the signal attenuation in response to the repetition of a stimulus. Cross-adaptation, in contrast, refers to the signal attenuation that is observed (at some cortical level) because the underlying neuronal process cannot effectively distinguish the difference of the stimuli and processes them as effectively similar. Two caveats have to be kept in mind while interpreting fMRI-adaptation results: First, fMRI-adaptation is a relative measurement. Even the baseline condition might have some degree of adaptation if it shares stimulus properties with the experimental conditions. Second, cross-adaptation can also occur along stimulus dimensions that are unpredictable by the experimenter (Sawamura et al., 2006). Therefore, the stimulus set as well as the control conditions have to be defined with utmost caution.

In a recent neuroimaging study Rauschenberger and colleagues (2006) made use of an fMRI cross-adaptation paradigm to reveal the neuronal substrate of amodal completion. A stimulus that elicits amodal completion ('pictorial-occlusion prime') was presented either for $100 \mathrm{~ms}$ ('brief exposure') or for $250 \mathrm{~ms}$ ('long exposure'). The stimulus was masked and then followed by either a stimulus that matched the physical properties ('notched-disk probe') or by a stimulus that matched the completed percept of that stimulus ('complete-disk probe'). Focusing on the temporal unfolding of the completion process, they showed that after brief exposure of the pictorial-occlusion prime the $\mathrm{fMRI}$ signal to the notched-disk probe was adapted, while at long exposure of the pictorial-occlusion prime the $\mathrm{fMRI}$ signal to the complete-disk probe was adapted.This 
effect was true for early (V1, V2, V3, V4v) as well as higher visual areas (lateral occipital complex, LOC; Malach et al., 1995). The authors conclude that with brief exposures only the representation of the physical stimulus properties is represented in all visual areas. In contrast, at long exposures the physical and the completed interpretation seem to be represented concurrently. Thus, this study is valuable in showing that the amodal completion process takes time to evolve. However, the study is inconclusive with respect to the question of the different roles of early and late cortical processing stages for the process of completion.

In order to address this issue we designed our study in a way that allowed us to directly compare brain activity related to the sensation of the physical properties of the stimulus and brain activity correlated with the perception of the completed shape. First, we used line drawings instead of filled shapes to maximize any possible adaptation effects for early visual cortex. It has been shown that fMRI-adaptation effects in early visual cortex can be elicited by small high-contrast black-and-white elements (Kourtzi and Huberle, 2005; Murray, Olman et al., 2006). Second, we presented the second stimulus - whether it was a physical or a completed interpretation - at the very same retinal location it was displayed as part of the stimulus eliciting amodal completion (see Figure 1 for exemplar stimuli and Additional Figure 1 for the complete stimulus set). Third, we did not use a masking stimulus because a masking stimulus might lead to additional, potentially conflicting processing of visual information. Fourth, we presented our stimuli for 300 ms to ensure that the stimuli were indeed amodally com-

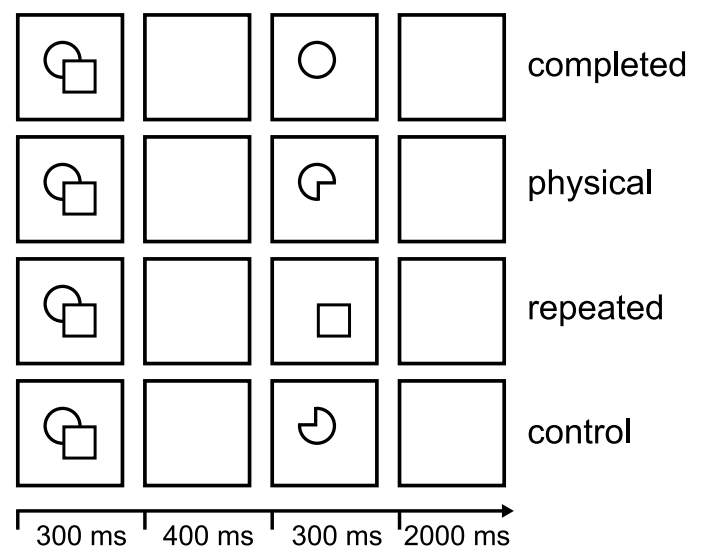

Figure 1. Experimental procedure. An experimental trial began with the presentation of one composite stimulus for $300 \mathrm{~ms}$, which was followed by a blank screen for $400 \mathrm{~ms}$. Then the test stimulus was presented for $300 \mathrm{~ms}$, followed by a blank screen for 2,000 ms. The test stimulus was either the completed shape ('completed'), the physical shape of the occluding stimulus ('physical'), a repetition of the occluding shape ('repeated'), or the physical shape rotated in plane around 180 degrees ('control'). All single-shape test stimuli were displayed at the exact positions they obtained in the composite stimulus shown before. Note that the composite stimulus - the stimulus that evokes amodal completion - is the same for all experimental conditions. The four experimental conditions are defined by the four different test stimuli. Thus, differences in fMRI signal are only based on differences between the test stimuli. 
pleted. It has been shown that at a presentation rate of $>200$ ms composite stimuli produce priming effects equivalent to their completed interpretation (Sekuler and Palmer, 1992).

More specifically, an experimental trial within our rapid event related design began with the presentation of one composite stimulus for $300 \mathrm{~ms}$, which was followed by a blank screen for $400 \mathrm{~ms}$ (see Figure 1). Then the test stimulus was presented for 300 $\mathrm{ms}$, followed by a blank screen for $2000 \mathrm{~ms}$. The test stimulus was either the completed shape ('completed'), the physical shape of the occluding stimulus ('physical'), a repetition of the occluding shape ('repeated'), or the physical shape rotated in plane around 180 degrees ('control'). Note that the composite stimulus - the stimulus that evokes amodal completion - is the same for all experimental conditions. The four experimental conditions are defined solely by the four different test stimuli. Thus, differences in fMRI signal are only based on differences between the test stimuli.

Following the logic of fMRI adaptation and cross adaptation described above we computed the contrast physical < completed in order to localize brain areas that process the physical shape (but not the amodally completed shape) and the contrast completed < physical in order to localize brain areas that process the completed percept (but not the physical shape). In other words, a brain region representing the physical stimulus should display a decreased (cross-adapted) fMRI signal when the physical shape follows the composite stimulus; in contrast, a brain region representing the completed percept should display a decreased (cross-adapted) fMRI signal when the completed shape follows the composite stimulus.

Using such an $\mathrm{fMRI}$-adaptation design we are able to demonstrate a differential effect for early visual cortex, presumably V1 in comparison to higher visual area LOC. We demonstrate that the physical features (local contours) of the stimulus are processed in primary visual cortex. In contrast, higher visual areas such as the LOC no longer represent the original contour constellations but only the segregated and fully completed shapes. We argue in favour of a multiple step model in amodal completion involving different cortical areas.

\section{Results and Discussion}

\section{Brain regions representing the completed stimulus}

To identify brain regions encoding the completed stimulus we computed the contrast completed < physical (see 'Methods' for a detailed description of data analyses). The group analysis revealed three regions in inferior temporal cortex (ITC), two in the left hemisphere and one in the right hemisphere (Talairach coordinates [Talairach and Tournoux, 1988]: left ITC posterior, x, y, z: -35, -78, -2; left ITC anterior, x, y, z: -47, -63, -4; right ITC, $x, y, z: 42,-66,-7)$. These regions showed significantly ( $p<0.05$, corrected) less increase of fMRI signal to the completed stimulus in comparison to the physical stimulus (adaptation). Figure 2 displays the contrast map superimposed on two brain slices (Figure 2A) and the event-related BOLD fMRI signal time courses of the corresponding regions (Figure $2 \mathrm{~B}$ ).

We performed additional analyses within these so defined regions-of-interest (ROls; see Methods). To test for the adaptation to the repetition of the occluding shape 
A

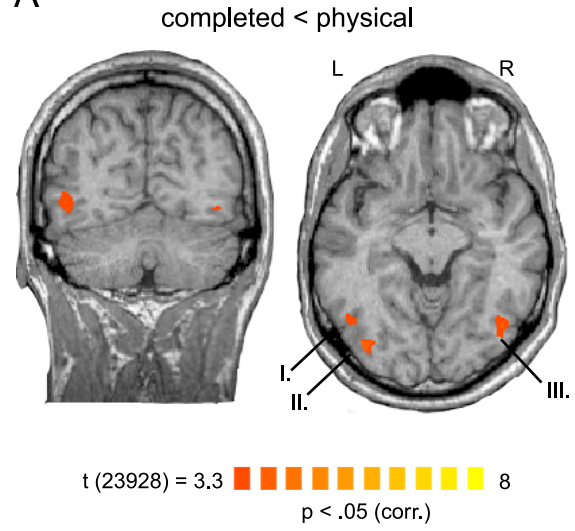

B
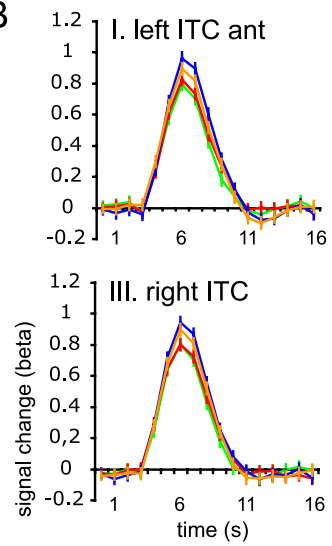

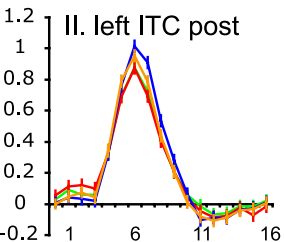

completed physical repeated control

Figure 2. Brain regions representing the completed stimulus. Panel A displays the contrast map averaged across subjects showing regions responding significantly less to the completed stimulus in comparison to the physical stimulus (contrast completed < physical; $\mathrm{p}<0.05$, corrected for multiple comparison using a cluster size threshold). The statistical map is superimposed on a coronal and an axial slice of one subject. Panel B displays event-related deconvolved BOLD fMRI responses (beta weights, averaged across trials and subjects) from the regions displayed in A reported against time for each of the experimental conditions: completed [red], physical [blue], repeated [green], and control [orange]. Time point 1 = trial onset. Error bars correspond to standard errors of the mean. Abbreviations: ITC, inferior-temporal cortex; post, posterior; ant, anterior.

('repeated') we computed the classical adaptation contrast repeated < control (note that this contrast is orthogonal to the ROI-defining contrast). We found that these regions demonstrate a significantly decreased fMRI signal to the repeated stimulus in comparison to the control stimulus (Table 1). This finding reassures us that the observed decreased fMRI signal to the completed stimulus in contrast to the physical stimulus is most likely based on crossadaptation. Furthermore, all three pre-defined regions displayed a significant adaptation effect for the completed condition in contrast to the control condition (Table 1). However, none of the contrasts physical < repeated, physical < control, completed < repeated showed a significant result (Table 1). We also computed the same contrast that defined the ROI (completed < physical) across voxels within the ROI (across time points). This contrast turned out to be significant at $p<0.000001$ (Note that the higher significance level is expected because the selection criteria was identical to this contrast). Furthermore, we calculated single-subject maps for the contrast completed < physical (Additional Figure 2). Since there are no activation patterns for this contrast observable in early visual areas we rule out the possibility that the lack of completion effects in early visual areas is due to individual differences. In sum, three regions in inferior temporal cortex displayed an adapted fMRI signal to the completed and the repeated and no adaptation to the physical and the control condition. We thus conclude that these regions represent the completed and the repeated stimuli. 
Table 1. ROI-based analysis

\begin{tabular}{llllllllll}
\hline & \multicolumn{2}{l}{ Left ITC, ant } & \multicolumn{2}{l}{ Left ITC, post } & \multicolumn{2}{l}{ Right ITC } & \multicolumn{2}{l}{ V1 } \\
\hline Contrast & $\mathrm{p}$ & $\dagger$ & $\mathrm{p}$ & $\dagger$ & $\mathrm{p}$ & $\dagger$ & $\mathrm{p}$ & $\dagger$ \\
\hline physical $<$ completed & - & - & - & - & - & - & $0.00^{*}$ & 3.65 \\
completed < physical & $0.00^{*}$ & 5.34 & $0.00^{*}$ & 6.7 & $0.00^{*}$ & 5.43 & - & - \\
physical < repeated & $0.00^{\S}$ & -7.61 & $0.00^{\S}$ & -6.35 & $0.00^{\S}$ & -6.05 & 0.85 & 0.19 \\
physical < control & $0.01^{\S}$ & -2.68 & $0.01^{\S}$ & -2.54 & $0.03^{\S}$ & -2.16 & 0.08 & 1.76 \\
completed < repeated & $0.02^{\S}$ & -2.27 & 0.73 & 0.34 & 0.48 & -0.70 & $0.00^{\S}$ & -3.46 \\
completed < control & $0.01^{*}$ & 2.66 & $0.00^{*}$ & 4.15 & $0.00^{*}$ & 3.18 & 0.06 & -1.88 \\
repeated < control & $0.00^{*}$ & 4.93 & $0.00^{*}$ & 3.81 & $0.00^{*}$ & 3.89 & 0.12 & 1.57 \\
\hline
\end{tabular}

Statistical values for different pairwise comparisons that were computed on the pre-defined ROls. Degrees of freedom are 23,928. ${ }^{*} p<0.05$ with $t>0 ;{ }^{\S} p<0.05$ with $t<0$. See Figures 2 and 3 and the results section.

Our results are in congruence with previous neuroimaging (Kourtzi and Kanwisher, 2001; Lerner et al., 2002), EEG (Murray et al., 2004), and MEG (Lerner et al., 2004; Liu et al., 2006) studies that demonstrated completion effects in higher visual areas. More precisely, these fMRI studies identified a region in inferior temporal cortex, namely the lateral occipital complex, LOC (Malach et al., 1995), to be involved in visual completion processes (Kourtzi and Kanwisher, 2001; Lerner et al., 2002). The reported Talairach coordinates suggest a perfect match to our results.

But beyond simply confirming that the LOC is involved in the completion process, our data suggest that the LOC represents the fully completed object exclusively. It was not in the scope of the current study to investigate cases when a composite stimulus is present, but no amodally completed shape is perceived (e.g. due to very short presentation times). That is, we can only say that in cases when amodal completion is perceived, the LOC represents the amodally completed object.

While previous investigations used shapes which where occluded by gratings (Kourtzi and Kanwisher, 2001; Lerner et al., 2002), we employed shapes which were occluded by shapes. In addition to previous neuroimaging studies our stimulus material allowed us to directly compare the amodally completed shape ('completed') with the actually presented shape ('physical'). Demonstrating fMRI adaptation to the amodally completed shape but not to the actually presented shape, we are able to conclude that at the level of LOC only the amodally completed percept is represented in the visual system. Information regarding the originally presented shape is no longer present. This finding corroborates the assumption by Sekuler and Palmer (1992) that the initial representation of the physical properties of the stimulus is not preserved as such at higher processing levels (at least not in the shape processing pathway) but is replaced by the representation of the amodally completed object.

Most notably, the adaptation effect to the completed stimulus relies only on that specific quarter of the stimulus which is actively completed. That is, the completed percept and the originally presented shape share three quarters of their areas and still they are processed fundamentally different in LOC.These data suggest that at the level 
of the LOC the local contour information available in the picture is no longer present but has been replaced by a representation of the completed percept. In sum, our data suggests that the LOC is the first stage in cortical processing where the fully completed object is represented.

\section{Brain regions representing the physical stimulus}

We performed the contrast physical < completed in order to localize brain regions processing the physical features of the stimulus but not the completed percept. Our group analysis yielded only one region in the calcarine sulcus of the left hemisphere (Talairach coordinates: $x, y$, and $z,-12,-90,-0)$ that showed a significantly $(p<0.01$, uncorrected) decreased fMRI signal to the physical stimulus in comparison to the completed stimulus (adaptation; Figure 3). Additional ROI-based analyses of this region revealed that the contrast physical < completed is significant at $p<0.0003$ (Table 1). Till now it has been a challenge to observe adaptation effects in primary visual cortex and successful paradigms employed rather long and intense adaptation phases (Fang et al., 2005). Since we wanted to address early as well as higher visual areas in one experiment, the parameters for our short-term-adaptation design had to be adjusted to suit both cortical stages. Thus, we expected the effects for the early visual areas to be smaller compared to other studies investigating V1. We also expected the adaptation effects in V1 to be smaller compared to the adaptation effects detected in higher visual areas which have been shown to be robust using a variety of paradigms (Grill-Spector et al., 1999; Grill-Spector and Malach, 2001; Kourtzi and Kanwisher, 2001; Lerner et al., 2002). However, taking into account that our experimental conditions differed only in a quarter of the area of the stimuli presented, the observed adaptation to the physical stimulus is striking. That is, three quarters of the contour and the area of the two stimuli (physical and completed) match completely and the observed adaptation effect can only be due to the fourth quarter being different. In this regard, the demonstrated adaptation effect is quite strong. Furthermore, we computed the contrast map repeated $<$ control and identified a region in the calcarine sulcus of the left hemisphere that displayed the classical adaptation effect. Both regions, i.e. the one showing adaptation to the repetition of the covering stimulus (in comparison to the control stimulus) and the one showing adaptation to the uncompleted physical stimulus (in comparison to the completed) are adjacent to one another and are both in early visual cortex, presumably V1 (Figure 3C).

We further speculated that high inter-subject variability of anatomical structures might be one cause for the general difficulty to observe adaptation effects in V1 and for our difficulty in particular to observe these effects in the right hemisphere. In order to have a more detailed look onto the observed adaptation effects in early visual cortex we conducted single-subject analyses to compare the individual results to the retinotopy. That is, we computed the contrast physical < completed for each individual subject and superimposed the resulting contrast maps onto the individually defined retinotopic maps (Figure 4). Most notably, in all of our subjects we identified regions in early retinotopic areas of both hemispheres that showed a significantly $(p<0.05)$ lower fMRI signal for the physical stimulus in contrast to the completed stimulus (adaptation). The exact localization of these regions, however, varied between subjects as expected due to the high intersubject variability of anatomical structures in the 
A

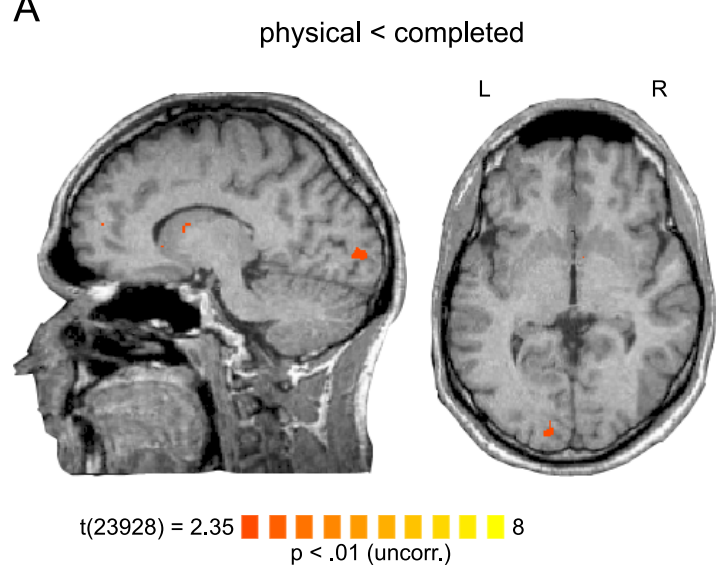

B left calcarine sulc.
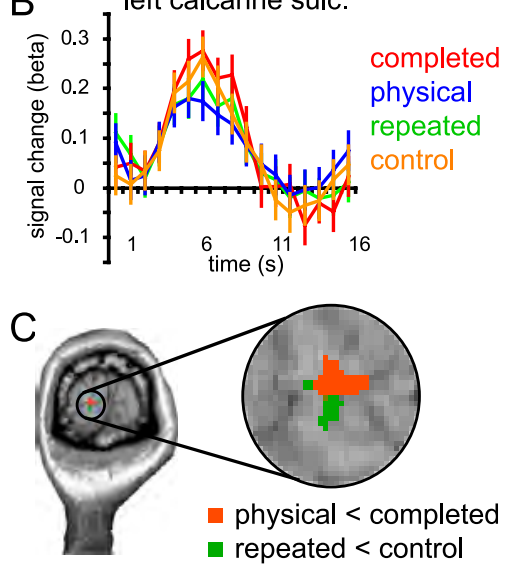

Figure 3. Brain region representing the physical stimulus. Panel A displays the contrast map averaged across subjects showing a region responding significantly less to the physical stimulus in comparison to the completed stimulus (contrast physical < completed; $p<0.01$, uncorrected). The statistical map is superimposed on a sagittal and an axial slice of one subject. Panel B displays the event-related deconvolved BOLD fMRI responses (beta weights, averaged across trials and subjects) from this region reported against time for each of the experimental conditions: completed [red], physical [blue], repeated [green], and control [orange]. Time point $1=$ trial onset. Error bars correspond to standard errors of the mean. Abbreviations: sulc, sulcus. Panel $C$ depicts two contrast maps superimposed on a coronal slice of one subject and a close-up of the activation pattern. The orange contrast map shows the same region as in panel A (contrast physical < completed). The green contrast map shows a region responding significantly less to the repeated stimulus in comparison to the control stimulus (contrast repeated < control).

occipital lobe. In four subjects the region was confined to the area representing the fovea, in the other four subjects the region was clearly confined to V1, however at a more eccentric localization. Smaller regions that showed a decrease of fMRI signal for the physical stimulus in contrast to the completed stimulus were also found in V2 (in four subjects bilateral, in two subjects only on one hemisphere). Additional activations were also found in other regions (see Figure 4: e.g. HDR01, right hemisphere and EWA29, left hemisphere, presumably V3A, V3B), however these varied substantially across subjects. Stimuli evoking amodal completion naturally also contain depth information, so we might speculate that also areas processing depth cues are involved in representing these stimuli (e.g. areas of the dorsal pathway, V3a, Tsao et al., 2003). Further investigations are needed to clarify this issue.

Thus, the high inter-subject variability explains in part the small effects in our group-analysis. In sum, the single-subject analysis confirms our group-analysis on a more powerful level in detecting adaptation effects in early visual cortex. Here we provide neuroimaging evidence for the processing of the physical features of a stimulus that induces amodal completion. We found regions in early retinotopic areas to demonstrate adaptation of activation to the physical stimulus but not to the completed stimulus (both following a composite stimulus that elicits amodal completion). Im- 
portantly, we demonstrate that these regions in early visual cortex also show a clear adaptation effect to the repeated stimulus but no adaptation to the control stimulus (both following a composite stimulus that elicits amodal completion). Since the control stimulus was identical to the physical stimulus but rotated in plane, we conclude that early visual cortex encodes the local contours of the stimulus that induces amodal completion.

Since we presented our composite stimuli in the center of the visual field, the exact localization with respect to early visual areas V1/V2 seems difficult. Nevertheless, our data fits well into a current working model of area $\mathrm{V} 1$ : One of the major functions of

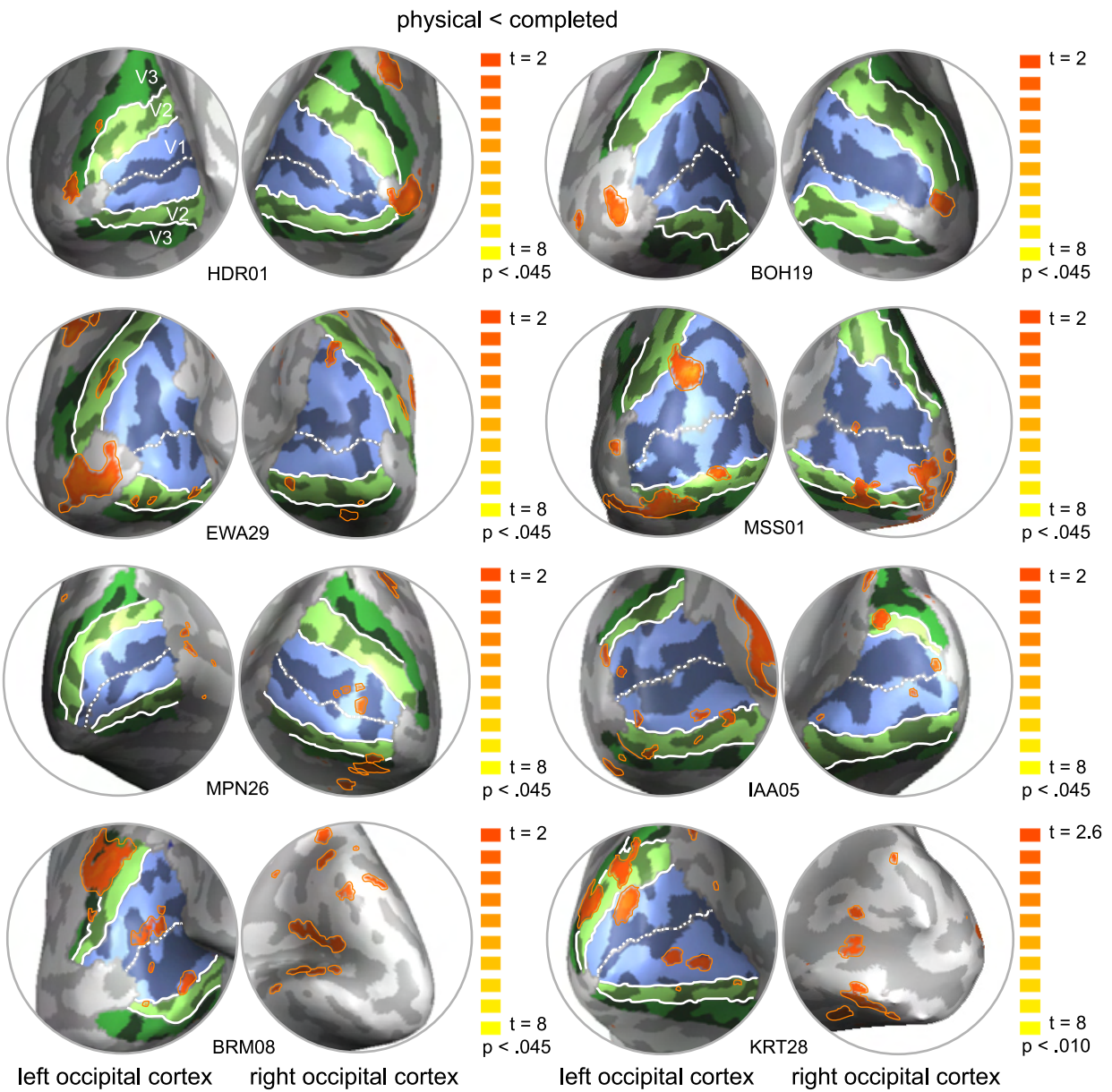

Figure 4. Single-subject analysis and retinotopic mapping. Medio-posterior views on the inflated left and right occipital cortex of eight subjects are shown. Retinotopic areas (V1 [light blue], V2 [light green], V3 [dark green]) and individual contrast maps (contrast physical < completed [orange]; $p<0.045$; degrees of freedom $=2,666$ ) are superimposed. 
this area is decomposing the visual field into short line segments of different orientations. Line segments carry information about edges and contours. Thus, V1 is said to provide an initial two-dimensional approximation of the shape of a stimulus. We are confident that our data supports the conclusion that the local contours of the stimulus that induces amodal completion are represented in V1.

In the following text passage we will discuss our findings in comparison to the study by Rauschenberger et al. (2006). While this study investigated amodal completion with a focus on temporal dynamics of the completion process using two different presentation times, we used only one presentation time (300 ms). Thus, we are only able to compare our results to the ones obtained by Rauschenberger et al. with the long exposure of 250 ms. In congruence with Rauschenberger et al. (2006) we find early as well as higher visual areas to be involved in the processing of stimuli eliciting amodal completion. However, our study allows us to extend this conclusion by demonstrating a differential functional profile for early (V1) in comparison to higher (LOC) visual areas. While Rauschenberger et al. (2006) found evidence for a representation of the notched and the completed shape concurrently in all (early and late) visual areas, we were able to reveal a distinctive pattern for early in contrast to late visual areas. In contrast to Rauschenberger we used high-contrast line drawings instead of red/green squares and we presented the test stimuli at the exact retinal location they obtained in the composite stimulus. Both manipulations were chosen to optimize the visual presentation for early visual areas. Furthermore, Rauschenberger et al. (2006) used a strong visual backward masking. To our knowledge, it is not known at which levels of processing the mask leads to interferences with the process of amodal completion. Also their probe stimuli consisted of composite stimuli that again triggered the process of amodal completion. Our test stimuli were designed according to the original psychophysical work by Sekuler and Palmer (1992), and triggered no additional process of completion. Further differences between the Rauschenberger study (2006) and our study concern e.g. magnetic field strength (they 1.5 T, we $3 \mathrm{~T}$ ) and number of trials per condition (they 48, we 144). In sum, we think that we have carefully selected parameters in order to optimize for statistical power and we have now succeeded in showing a differential effect between V1 and LOC on the basis of our different stimulation procedure.

\section{The process of amodal completion}

One reasonable interpretation of our data suggests that amodal completion is a process that involves distinctive different processing in two cortical stages: first, the detection and processing of the local contour information of the stimulus without the completion and second, the representation of the fully completed shape without the representation of the original stimulus. That only the physical features of an occluded object are initially present in the visual system was first suggested by Sekuler and Palmer (1992). They also speculated that this initial representation must be linked to its specific retinal location. Our findings corroborate this assumption. By demonstrating that the physical features of the stimulus are encoded in retinotopic areas of the visual brain we confirm this first prediction. Furthermore, in their comprehensive study, Sekuler and Palmer (1992) demonstrated that completed interpretations of composite stimuli can be primed at different positions within the visual field. Thus, they concluded 
that in a later stage of processing where representations can be more spatially invariant the completed objects are represented (irrespective of their position in the visual field). We show that the fully completed object is represented in the LOC. Moreover, in previous neuroimaging studies, it was found that this area encodes perceived shapes irrespective of their position in the visual field (Grill-Spector et al., 1998; 1999; Tootell et al., 1998a). It still needs to be directly tested whether also amodally completed objects are represented in the LOC in a position invariant fashion.

If early visual cortex, presumably $\mathrm{V} 1$, processes the initial local contour of the stimulus and the fully completed object is encoded in LOC, where does the actual completion process occur? We can only speculate on this issue: After detecting the local contours the basic task for the visual system is to assign the contours to the different objects. In case of occlusion the occluding contour has to be assigned to the occluding object and not to the object being occluded. The missing contour of the object being occluded can then be completed based on Gestalt rules. Our experiment was not designed to identify this complex computational process. We rather focused on the start and end points of the completion process. Thus, based on our data we are not able to make any inferences about where the computations occur. However, we would like to mention results proposing that this computational effort might take place in area V2. Supporting evidence for this interpretation stems from recent studies using single cell recordings in monkeys (Bakin et al., 2000; Qiu and von der Heydt, 2005; Zhou et al., 2000) and work by Zhaoping (2005) introducing a corresponding computational model. The electrophysiological investigations demonstrate that the problem of border ownership is resolved in visual area V2 (Bakin et al., 2000; Zhou et al., 2000). Area V2 combines stereoscopic cues with Gestalt rules to represent the borders of 2D figures as if the figures were objects in 3D space (Qiu and von der Heydt, 2005). Zhaoping (2005) suggests that these computations are based on intracortical interactions in area V2. Furthermore, Bakin and colleagues (2000) found that neurons of area V2 signal partially occluded contours by flank facilitation. Furthermore, it has been shown that area V4 is crucial for midlevel form vision and contour integration, and that V4 lesions impair the discrimination of illusory borders (Gallant et al., 2000). Thus, this area of the ventral visual pathway might also integrate the amodally completed contours. However, there is psychophysical evidence that amodal completion does not only involve contour completion, but also surface and even volume completion (Tse, 1999), which appears to be solved at the level of the LOC.

Our data, taken together with previous findings, are in line with the interpretation of a feedforward model for the cortical processes underlying amodal completion: First, local contour information is extracted and processed in primary visual cortex. Second, real contours are assigned to different objects in area V2 and missing contours are filled in on the basis of basic Gestalt-rules (probably V2/V4). Finally, the fully completed object is represented in the LOC. The model suggests a serial order of processing steps. Evidence for such a serial processing comes from behavioural experiments (Rauschenberger and Yantis, 2001; Sekuler and Palmer, 1992) as well as recent MEG studies (Liu et al., 2006; Plomp et al., 2006). Using different approaches such as priming and a visual search task, both behavioural investigations agree that the representation of the completed shape is preceded by a representation of the local contour information (Rauschenberger and Yantis, 2001; Sekuler and Palmer, 1992). This finding is cor- 
roborated by the MEG studies revealing shorter latencies for a primed stimulus that contains only the local contour information of a composite stimulus in contrast to a primed completed shape (Liu et al., 2006; Plomp et al., 2006). Another MEG study investigated contextual influences in amodal completion (de Wit et al.,2006). Also this study found earlier components to be primarily responsive to physical stimulus attributes, while later components resembled the integration of physical stimulus attributes to global objects.

Thus, a feedforward model can explain our results, however, also more complex models involving feedback connections (Murray et al., 2004) are still under debate that also give credit to the finding that even higher cognitive functions (i.e. visual short term memory) can interfere with the process of amodal completion (Lee and Vecera, 2005). In conclusion, our data suggests that the initial representation of the physical properties of the stimulus might still be preserved at the level of $\mathrm{V} 1$ but are ultimately replaced at the higher processing level of LOC.

\section{Conclusion}

In the present study we provide neuroimaging evidence for a two stage model of amodal completion. Using fMRI adaptation we are able to demonstrate that the physical features (local contours) of the stimulus eliciting amodal completion are processed in early visual cortex, presumably V1. In contrast, higher visual areas such as the LOC do not represent the original contour constellations but only the segregated and fully completed shapes. Our data are in line with a serial process evolving over time although the temporal evolvement was not the focus of this study. In sum, our study complements psychophysical investigations and electrophysiological findings towards a more complete picture of the cortical processes underlying amodal completion.

\section{Methods}

\section{Subjects}

Ten human subjects (aged 17-32 years, mean 27 years) with normal or corrected-tonormal vision took part in this study. All gave their informed written consent to the procedure in accordance with institutional guidelines and the Helsinki declaration (Declaration of Helsinki [http://www.wma.net/e/ethicsunit/helsinki.htm]. Each subject participated in the amodal completion study and also underwent standard retinotopic mapping to assess the boundaries of retinotopic cortical areas V1, V2 and V3 (see Muckli et al., 2005; 2006 for a detailed description of the procedure).

\section{Stimulus material}

For the amodal completion study three different geometrical shapes - line-drawings of a circle, a square, and a diamond - formed the basis of the stimulus set. By pairing two of these shapes various composite stimuli were created (see Additional Figure 1 for the complete stimulus set). In total, twenty-four different composite stimuli were created. In twelve of the stimuli the occluding shape was a complete shape (line one, 
Additional Figure 1) and in the other twelve stimuli the occluding shape was a notched shape (line three, Additional Figure 1).This manipulation was introduced to leave room for the interpretation of the occluded stimulus. Importantly, since the physical stimulus was always a notched stimulus and the amodally completed stimulus was always a completed stimulus, we thus made sure that the occluding stimulus (the 'repeated') was in half of the cases similar to the physical stimulus and in the other half similar to the completed stimulus.

Furthermore, thirty-six different stimuli were created depicting line-drawings of single shapes (lines 4-8, Additional Figure 1). For each composite stimulus four different single-shape stimuli were created that served as test stimuli (see Figure 1). The stimuli were displayed at the centre of the screen as black line-drawings on a white square subtending $300 \times 300$ pixels (approximately five degrees of visual angle). The background of the screen was set to gray. Throughout the whole experiment a red fixation cross was present at the centre of the screen and subjects were instructed to maintain fixation. The fixation was small enough (subtending approx. 0.5 deg visual angle) not to overlap with the contours of the stimuli.

\section{Experimental procedure}

Visual stimuli were delivered under computer control using custom-made software (StimulDX, Brain Innovation, Maastricht, The Netherlands) to a high-luminance projector. The image was projected onto a screen that was fixed to the head coil and could be viewed by the subjects through a tilted mirror. Each experimental run began and ended with a fixation period of $10 \mathrm{~s}$. The experimental block in between consisted of 36 experimental trials per condition and 36 fixation trials. For a detailed description of an experimental trial see 'Background' and Figure 1. Subjects performed four experimental runs within one scanning session. Thus, each experimental condition was presented 144 times total. The order of presentation of the experimental trials was counterbalanced across runs per subject. That is, trials from each condition (fixation included) were preceded ( 2 trials back) equally often by trials from each other condition.

\section{FMRI data acquisition}

Blood oxygenation level-dependent fMRI (Ogawa et al., 1990) was performed with a standard birdcage head coil on a 3 T Siemens Trio Scanner (Siemens, Erlangen, Germany) at the Brain Imaging Center, Frankfurt, Germany. A gradientrecalled echo-planar imaging sequence was used with the following parameters: 16 axial slices; repetition time $(T R)=1,000 \mathrm{~ms}$; echo time $(T E)=40 \mathrm{~ms}$; flip angle $(F A)=60$ deg; field of view (FOV) $=210$; slice thickness $=5 \mathrm{~mm}$; inplane resolution $=3 \times 3 \mathrm{~mm}^{2}$; gap thickness $=0.5 \mathrm{~mm}$. Slices were oriented to achieve a total coverage of the occipital and temporal lobes. Each scanning session included the acquisition of a high-resolution magnetizationprepared rapid-acquisition gradient echo sequence $(\mathrm{TR}=2,250 \mathrm{~ms} ; \mathrm{TE}=2.62 ; \mathrm{FA}=9$ deg; FOV $=256$; slice thickness $=1 \mathrm{~mm}$; In-plane resolution $=1 \times 1 \mathrm{~mm}^{2}$ ) for coregistration and anatomical localization of functional data.

\section{Data analysis}

Data analysis and visualization was performed using the BrainVoyager QX software package (Brain Innovation, Maastricht, The Netherlands). The first four volumes of each event-related run were discarded to preclude T1 saturation effects. Pre-processing of 
the functional data included motion correction, linear trend removal and temporal highpass filtering at $0.01 \mathrm{~Hz}$, and slice-scan-time correction. Functional 3D data was spatially filtered employing a Gaussian filter with 8-mm kernel for the group analysis only. One subject had to be excluded from further analyses due to strong head movements. For each subject the functional and structural 3D data sets were transformed into Talairach coordinate space (Talairach and Tournoux, 1988). The recorded high-resolution anatomical scans were used for surface reconstruction.

Our rapid event-related fMRI study used closely spaced trials, leading to a substantial overlap in the resulting hemodynamic responses. Nevertheless, when the precautions of a balanced randomization are taken (two-back randomization, see above), the underlying hemodynamic responses can be assessed by deconvolution (Dale and Buckner, 1997). A deconvolution analysis estimates the hemodynamic response function for each trial on the basis of a general linear model (GLM). Twenty predictors (one predictor per volume) were defined to cover the temporal extent of a typical hemodynamic response. Statistical analyses were conducted volume-based and included a multi-subject fixed-effects GLM as well as single-subject fixed-effects GLMs. All GLMs were computed on the peak points of the fMRI-signal. GLMs were corrected for serial correlation and normalized to percent signal change. That is, the time course of a voxel or a region-of-interest is normalized in such a way that the mean signal value is transformed to a value of 100 with the individual values fluctuating around the mean as percent signal deviations. Thus, the reported signal change beta weights directly provide an estimate of the actual percent signal change.

For the statistical map completed < physical we performed a cluster-size thresholding to correct for multiple comparisons (Forman et al., 1995; Thirion et al., 2007). We employed a plugin for Brain Voyager QX by Fabrizio Esposito (Brain Innovation, Maastricht, The Netherlands) that makes use of a Monte-Carlo simulation (1000 iterations). The original map was thresholded at $p=0.000968, t(23,928)=3.3$. The estimated cluster size threshold was 322 voxels $\left(1 \times 1 \times 1 \mathrm{~mm}^{3}\right)$. Consequently, the multisubject statistical map in Figure 2 is thresholded at $\mathrm{p}<0.05$, corrected for multiple comparisons.

For the statistical map physical < completed no correction for serial correlation was applied. Since to-date it has been a challenge to observe adaptation effects in primary visual cortex and successful paradigms employed rather long and intense adaptation phases (Fang et al., 2005). Since we wanted to address early as well as higher visual areas, parameters for our short-term-adaptation design had to be adjusted to suit both cortical stages. Thus, we expected the effects for the early visual areas to be small.

Following the logic of fMRI adaptation described in the 'Background' we computed the contrast physical < completed in order to localize brain areas that process the physical shape (but not the amodally completed shape) and the contrast completed $<$ physical in order to localize brain areas that process the completed percept (but not the physical shape). In other words, a brain region representing the physical stimulus should display a decreased (adapted) fMRI signal when the physical shape follows the composite stimulus; in contrast, a brain region representing the completed percept should display a decreased (adapted) fMRI signal when the completed shape follows the composite stimulus. To ensure that the identified adaptation profile is truly based on 'adaptation' and not simply a lower fMRI signal we looked at the response profiles 
of the pre-defined regions in more detail. First, we computed the contrast repeated < control. This contrast is also called the basic adaptation contrast since it tests whether the identified region displays a conventional adaptation effect to a repeated stimulus. Second, we additionally computed the contrasts physical < repeated, physical < control, completed < repeated, and completed < control. Third, we also computed the same contrast that defined the ROI also within the ROI - however, since this computation is not statistically independent, it is only of descriptive use. We report t statistics, degrees of freedom and $p$ values (Table 1). 


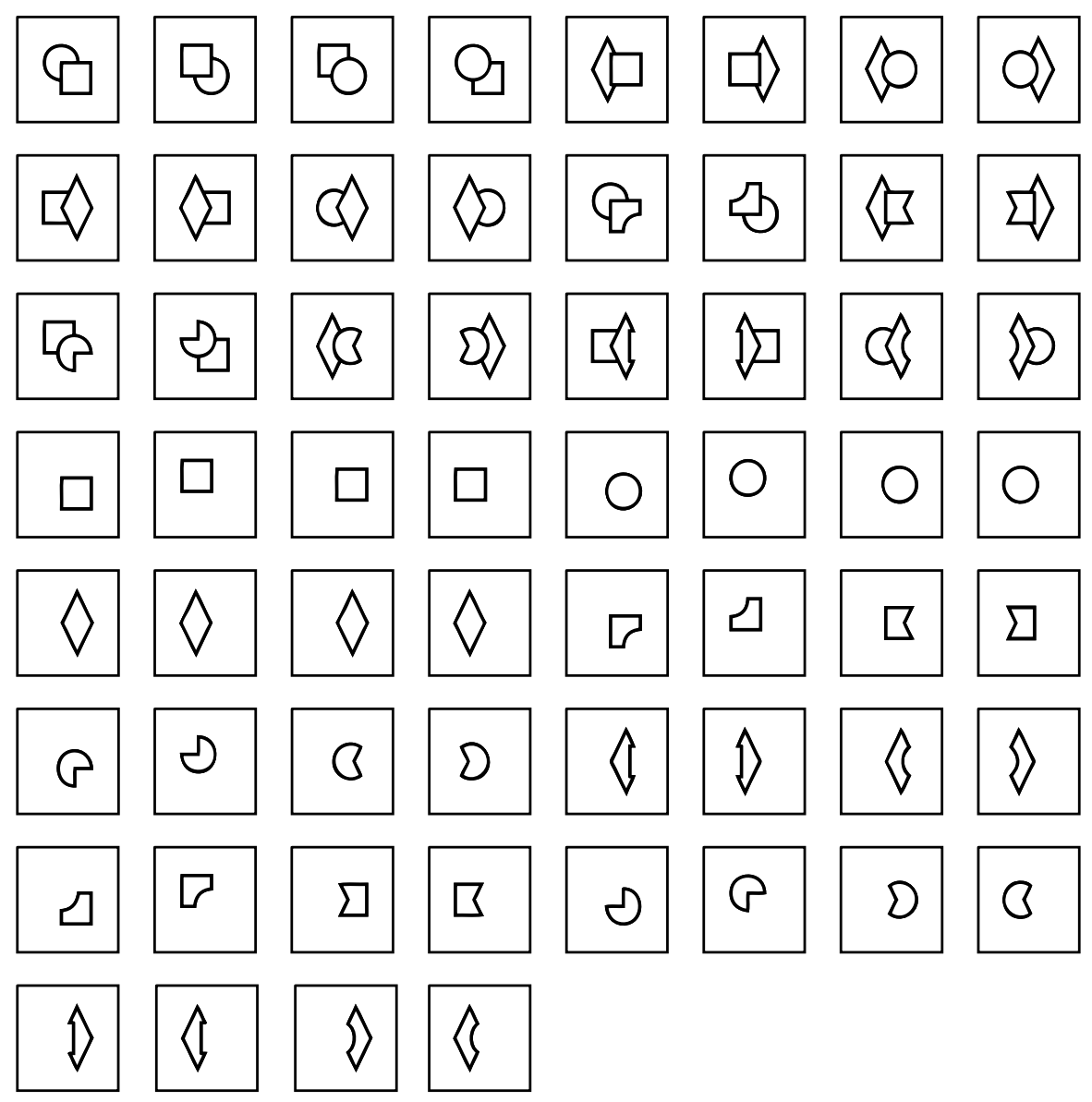

Additional Figure 1. The complete stimulus set. The complete stimulus set comprising all twentyfour composite (lines 1 to 3 ) and thirty-six test stimuli (lines 4 to 8 ) are presented. 


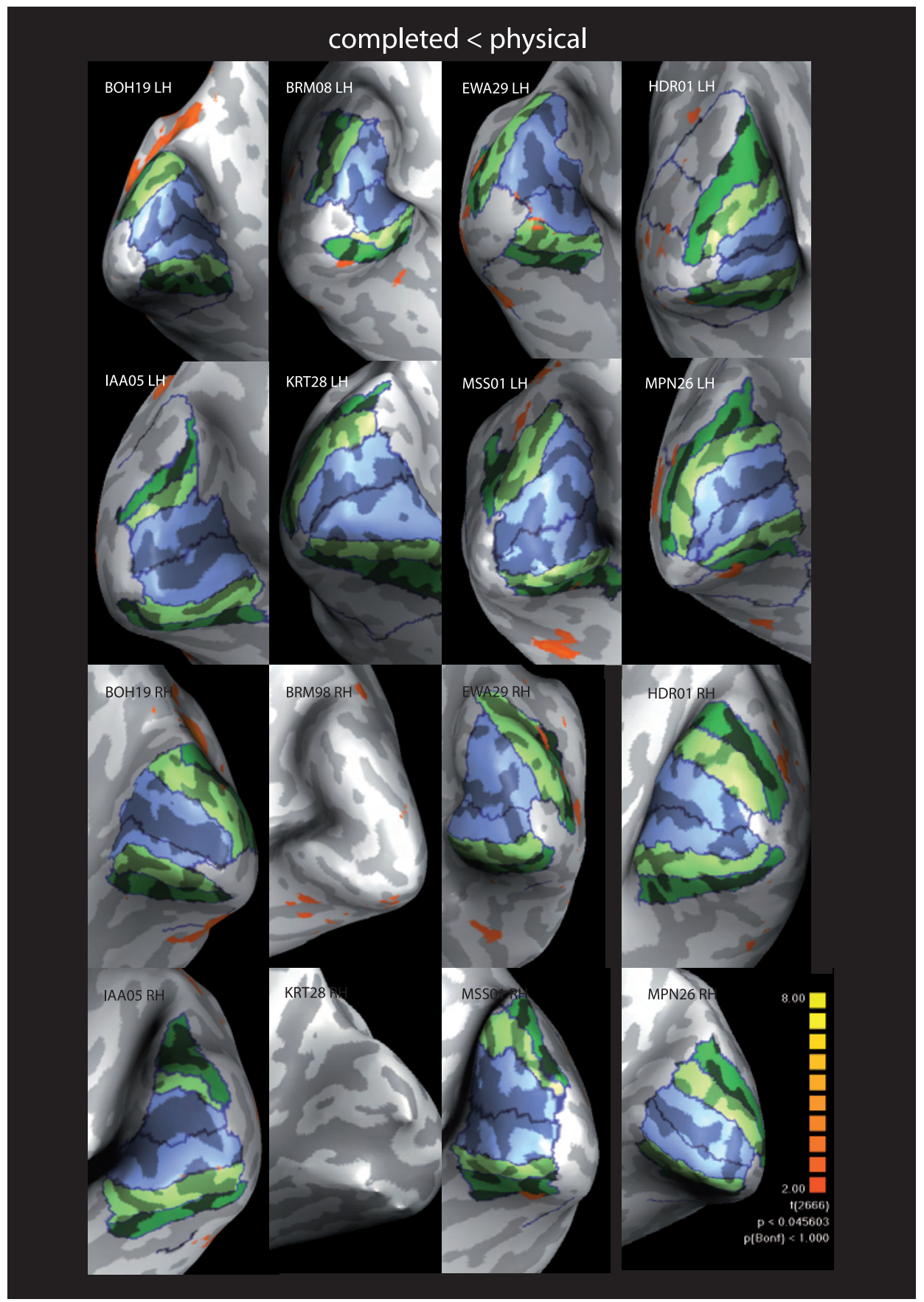

Additional Figure 2. Individual maps for the contrast completed < physical. Medio-posterior views on the inflated left and right occipital cortex of eight subjects are shown. Retinotopic areas (V1 [light blue], V2 [light green], V3 [dark green]) and individual contrast maps (contrast completed $<$ physical [orange]; $\mathrm{p}<0.045$; degrees of freedom $=2,666$ ) are superimposed. 



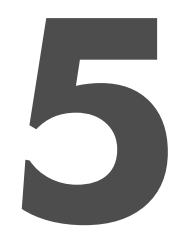

\section{Patterns of $\mathbf{f M R I}$ activity representing perceived di- rection in apparent motion}

The so-called 'motion quartet' is an apparent-motion stimulus that can be seen either in vertical or horizontal motion. Conscious experience switches spontaneously between the two possible interpretations without any changes in the physical characteristics of the stimulus. When brain activity is measured with fMRI during the switches between subjective states, a number of areas spanning multiple levels of the cortical hierarchy show switch-related activity. The question remains at which of those levels the content of our visual experience is actually determined, since other factors like attention- and motor-related processes are confounded with pure percept-related activity. In our study, we investigated areas that show activity related to perceptual switches during presentation of an array of motion quartets. We used multivariate techniques to detect areas that contain information about the perceived motion direction. Highest decoding performance was found in inferior frontal gyrus (IFG), right premotor cortex (PMC), and the temporoparietal junction (TPJ), as well as areas surrounding hMT/V5+ and early visual areas (V1-V3). The results suggest that multiple areas in visual as well as fronto-parietal areas encode motion direction of an ambiguous motion stimulus.

Kohler, A., Weigelt, S., Muckli, L., Singer, W., and Kriegeskorte, N. (in preparation). Patterns of fMRI activity representing perceived direction in apparent motion. 


\section{Introduction}

Studies in humans and non-human primates have identified the middle temporal (MT) area as an essential node in the cortical processing of motion (Allman and Kaas, 1971; Dubner and Zeki, 1971; Newsome et al., 1989; Tootell et al., 1995; Van Essen et al., 1981; Zeki et al., 1991). Microstimulation in this area influences the perceived direction of motion (Salzman and Newsome, 1994; Salzman et al., 1990; 1992) and interference with MT processing using transcranial magnetic stimulation or through lesions reduces the performance of human observers during direction discrimination (Baker et al., 1991; Beckers and Hömberg, 1991; 1992; Hotson et al., 1994; Newsome and Paré, 1988; Sack et al., 2006; Zihl et al., 1983). MT (or 'hMT/V5+' in humans) is not the only area exhibiting directional selectivity. Starting from primary visual cortex (V1), several areas in visual cortex show motion sensitivity at different spatial scales (Dupont et al., 1997; Hubel and Wiesel, 1968; Tootell et al., 1997). Also in parietal cortex, a number of motionselective areas have been described (Claeys et al., 2003; Sunaert et al., 1999).

In recent studies it could be shown that hMT/V5+ is especially vital for the conscious perception of motion, even if stimuli remain physically constant (Muckli et al., 2002; Sterzer et al., 2002). One paradigm often used in this context is apparent motion, where a perception of illusory motion is induced by a sequence of static images. hMT/ $\mathrm{V} 5+$ is more active during motion periods when subjective experience of an ambiguous stimulus switches between static flicker and apparent motion (Muckli et al., 2002). It also becomes transiently activated when perception switches between two possible interpretations of the so-called 'motion quartet' (Muckli et al., 2005; Sterzer and Kleinschmidt, 2005; 2007; Sterzer et al., 2002). The motion quartet consists of two pairs of diagonally opposite dots at the corners of a virtual rectangle (Neuhaus, 1930; Schiller, 1933; see Fig. 1).When the two pairs of dots are presented in alternation, either vertical or horizontal motion can be perceived and during prolonged viewing the perceived motion direction can spontaneously switch between the two possible interpretations. Apart from hMT/V5+, a network of areas showing responses to perceptual switches has been described in previous studies, ranging from early visual cortex to parietal and frontal areas (Sterzer and Kleinschmidt, 2005; 2007; Sterzer et al., 2002; 2003).

In our experiments, we wanted to further investigate this network of areas to find out in which of those regions the actual content of conscious perception during viewing of the motion quartet is represented. We used an array of motion quartets and participants had to report perceived motion direction while we measured brain activation using functional magnetic resonance imaging (fMRI). Since observers are required to report every perceptual switch by button presses, activation related to the representation of perceived motion is confounded with attentional and motor aspects. Univariate analyses cannot differentiate between perceptual states (vertical vs. horizontal motion) since switches in either direction are accompanied by similar amplitude changes in the $\mathrm{fMRI}$ signal. Therefore, we applied a multivariate approach to fMRI data analysis that has recently been described in the literature (Haynes and Rees, 2005a; Kamitani and Tong, 2005; Kriegeskorte et al., 2006). This approach uses the fine-grained pattern of activation across imaging voxels to decode information related to experimental conditions. In this way, we were able to identify cortical areas in which the conscious experience of the observers was reflected in the spatial pattern of $\mathrm{FMRI}$ responses. 


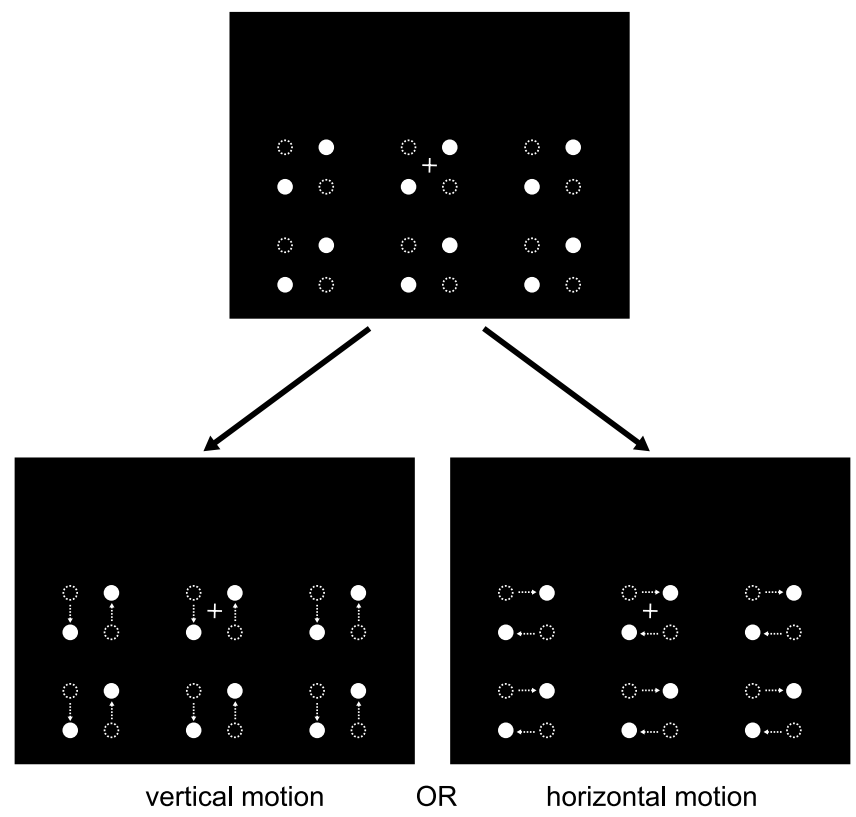

Figure 1. Stimulus design. The diagonally opposing pairs of dots (filled and dashed) were presented in alternation, leading to a percept of either vertical or horizontal motion. During prolonged viewing, the perceived motion direction oscillated between the two possible interpretations. The stimulus consisted of six virtual rectangles (or motion quartets); three in each row. Due to the head-coil geometry that limited the viewable screen, an upper row of stimuli was not presented.

\section{Methods}

\section{Participants}

Four healthy human observers (one female, mean 28,8 years) with normal or corrected-to normal vision participated in our experiments. All gave their written informed consent in accordance with institutional guidelines. Each participant took part in the apparent motion study and also underwent standard retinotopic mapping and a mapping session to localize hMT/V5+ (Muckli et al., 2005).

\section{Stimulus}

Stimuli were generated with a custom-made program based on the Microsoft DirectX library. The stimulus consisted of six virtual rectangles (or motion quartets); three in each row (Fig. 1). Each motion quartet was constructed by four circles (diameter, 1.07 deg). At any give time, only two dots at diagonally opposite corners were presented. A fixation cross (size, $0.57 \mathrm{deg} \times 0.57 \mathrm{deg}$ ) was always displayed in the middle of the screen. Note that, due to the head-coil geometry that limited the viewable screen, an upper row of stimuli was not presented. The horizontal and vertical distance between 
dots of one motion quartet was $5.71 \mathrm{deg}$. Motion quartets were separated from each other by 17.12 deg (center to center). Dots were presented for $250 \mathrm{~ms}$ with an interstimulus interval of $100 \mathrm{~ms}$ ( $60 \mathrm{~Hz}$ presentation frequency).

\section{Experimental Procedure}

While lying in the scanner in supine position, subjects viewed a translucent screen at the end of the head coil through a tilted mirror. Visual stimuli were delivered under computer control using custom-made software (StimulDX, Brain Innovation, Maastricht, The Netherlands) to a high-luminance projector (Sanyo Pro xtraX PLC-XP41 multiverse projector) that projected the image onto the translucent screen. The projected screen size subtended 57.05 deg $\times 42.79$ deg of visual angle.

Each experimental run began with a fixation period of $16 \mathrm{~s}$ and ended with a fixation period of $14 \mathrm{~s}$. The experimental block in between was of $180 \mathrm{~s}$ duration. Participants performed 10 experimental runs within one scanning session. Observers' responses were recorded via an optic-fiber response box. During the presentation of the motion quartet, observers had to indicate their current percept (vertical or horizontal motion) by continuously pressing one of two buttons with their right index and middle fingers. The response mapping between perceived motion direction and finger was reversed in each run, e.g., on even runs horizontal motion was reported with a button press of the right index finger while vertical motion was reported with a button press of the right middle finger. On uneven runs it was the opposite. Thus, the response type was not confounded with perceived motion direction.

\section{fMRI Data Acquisition}

Blood-oxygenation-level-dependent fMRI (Ogawa et al., 1990) was performed on a 3 Tesla Siemens Trio Scanner (Siemens, Erlangen, Germany) at the Brain Imaging Center Frankfurt am Main, Germany. Using a standard birdcage head coil, a gradient-recalled echo-planar imaging sequence was applied with the following parameters: 33 axial slices; repetition time $=2,000 \mathrm{~ms}$; echo time $=60 \mathrm{~ms}$; slice thickness $=3 \mathrm{~mm}$; in-planeresolution $=3 \times 3 \mathrm{~mm}^{2}$, gap size: $0.3 \mathrm{~mm}$. Slices were oriented to cover the whole brain. Each scanning session included the acquisition of a high-resolution magnetizationprepared rapid-acquisition gradient echo sequence (isotropic voxel size, $1 \mathrm{~mm}^{3}$ ) for coregistration and anatomical localization of functional data. Cortex reconstructions of all subjects were performed for visualization of the retinotopic maps and delineation of visual areas.

\section{Data Analysis}

Data were analyzed using the BrainVoyager QX 1.8.4 software package (Brainlnnovation, Maastricht, The Netherlands). The first four volumes (8 s) of each scan were discarded to allow for T1 saturation. Preprocessing of the functional data included: threedimensional motion correction, linear-trend removal, temporal high-pass filtering at $0.01 \mathrm{~Hz}$, and slice-scan-time correction with sinc interpolation.

\section{General Linear Model}

To specifically capture activity related to perceptual switches, a general linear model (GLM) was computed by, first, indexing every switch through a boxcar predictor of 4 
s per switch separately for reports of horizontal and vertical motion (the width of the boxcar was estimated from preliminary analyses). Those predictors were then convolved with a gamma-variate hemodynamic response function (Boynton et al., 1996; delta $=2.5$, tau $=1.25$ ). To identify the cortical network showing switch-related activity, both predictors (for vertical and horizontal motion) were contrasted with fixation baseline.

\section{Multivariate Pattern Analysis}

The main goal of our study was to find cortical areas whose response pattern would allow us to distinguish between states in which subjects perceived either vertical or horizontal motion. We used searchlight-based multivariate techniques similar to methods previously established in the recent literature (Haynes et al., 2007; Kriegeskorte et al., 2006). Searchlight-based methods perform multivoxel pattern analysis (MVPA) in the neighborhood of every measured voxel. MVPA was performed using custom-made code programmed in Matlab 7.5.0.342 (The MathWorks Inc., Natick, US).

In a first step, the searchlight MVPA was performed separately for each participant. For every voxel, we considered a spherical cluster of voxels in the vicinity (33 voxels overall). The data were split in two halves. On the first half (A), a GLM was used to determine the response pattern for the two conditions (vertical and horizontal motion) across voxels (one beta estimate per condition per voxel). The GLM residuals were used to compute an optimized error covariance matrix across voxels (Kriegeskorte et al., 2007). With the beta estimates and covariance matrix, the Fisher dimension for optimal separation of conditions in data set $A$ was determined. Then, the second half of the data (B) was projected on this dimension. This was done by computing a GLM for the second half. The resulting beta estimates were projected on the Fisher dimension and subtracted, providing a measure of the mean difference in classification (negative values would indicate classification in the wrong direction). This mean difference was normalized by the standard error of the GLM residual variance of data set $B$ on the Fisher dimension derived from data set $A$, providing a t statistic for classification success. The procedure was performed in both directions $(A->B, B->A)$ and the mean of both $t$ values was taken as classification result for this voxel.

The resulting individual $t$ maps (one $t$ value per voxel per participant) were smoothed with a Gaussian kernel (FWHM, $8 \mathrm{~mm}$ ). A second-level analysis (summary statistics, i.e., $t$ test against zero-mean null hypothesis) was then performed across participants in standard Talairach space.

\section{Results}

Participants continuously reported whether they perceived vertical or horizontal motion during presentation of the motion quartets. We used a whole set of motion quartets to cover a large area of the visual field and also to emphasize global motion processing. In previous studies, it could be shown that the perceived motion direction in an array of simultaneously presented motion quartets is coherent across the visual field (Ramachandran and Anstis, 1983). All participants reported regular switches be- 

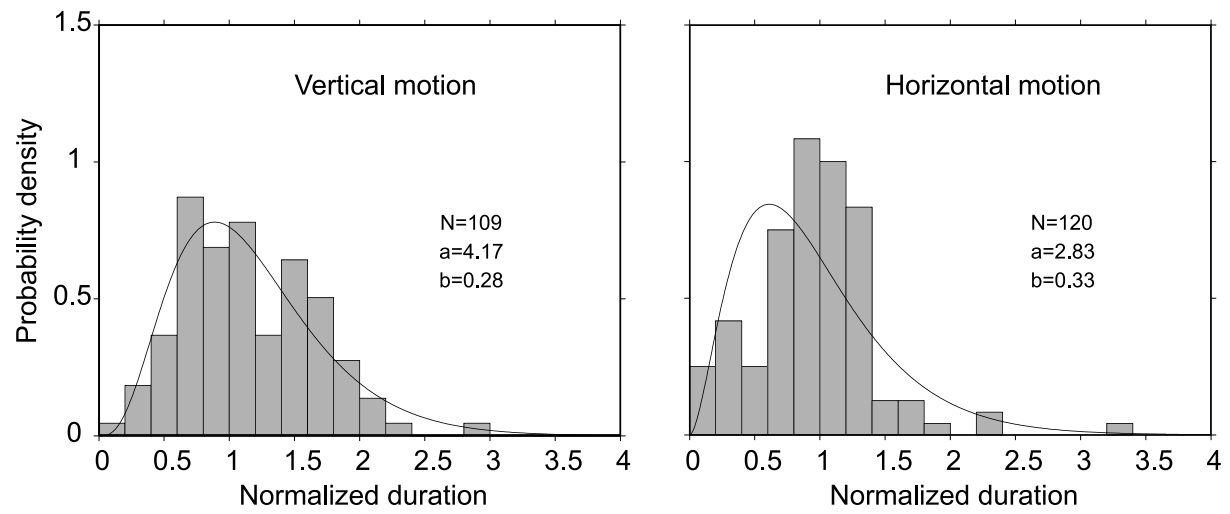

Figure 2. Distributions of phase durations. Histograms and best-fitting gamma distributions for perceived vertical and horizontal motion. For each participant, phase durations were first normalized to the median value separately for the two percepts. The parameters of the gamma distributions were calculated using maximum-likelihood estimates. $\mathrm{N}$, total number of phaseduration samples from all participants; $a$, shape parameter of the fitted gamma distribution; $b$, scale parameter of the fitted gamma distribution.

tween percepts. The median phase duration for vertical motion was $25.46 \mathrm{~s}$ and $22.78 \mathrm{~s}$ for horizontal motion. This difference in phase durations was not significant (Wilcoxon rank-sum test, $z=1.72, p=0.0851$ ). The distribution of phase durations could be approximated by gamma distributions (Fig. 2).

In a first step, a univariate analysis of activity related to perceptual switches was performed using a random-effects GLM. A network of areas showed switch-related activity, including early visual cortex, hMT/V5+, intraparietal sulcus (IPS), sensorimotor cortex (SMC), inferior frontal gyrus (IFG), and frontal operculum (Fig. 3). A similar network has been desribed in previous studies (Muckli et al., 2002; Sterzer et al., 2002; Sterzer and Kleinschmidt, 2007).

For the multivariate analysis, we used a searchlight-based approach (cf. Haynes et al., 2007; Kriegeskorte et al., 2006; 2007). For every voxel, the response pattern in a neighbourhood of 33 voxels (radius, $6 \mathrm{~mm}$ ) was considered for pattern classification with linear discriminant analysis. Classification was performed separately for participants and then combined in a summary statistics across participants. The aim of the analysis was to find areas containing information about the consciously perceived direction of motion in their fine-grained activity pattern. We found a prominent network of areas in right frontoparietal cortex showing significant classification accuracy, especially right IFG, premotor cortex (PMC), temporoparietal junction (TPJ), and frontopolar cortex (Fig.4). In the vicinity of hMT/V5+, which is the classical motion-selective area in human visual cortex, there was only one area in the left inferior occipital gyrus showing significant classification levels. In addition, early visual areas, predominantly V1, showed effects in the pattern analysis at a lower threshold (Fig. 5). 


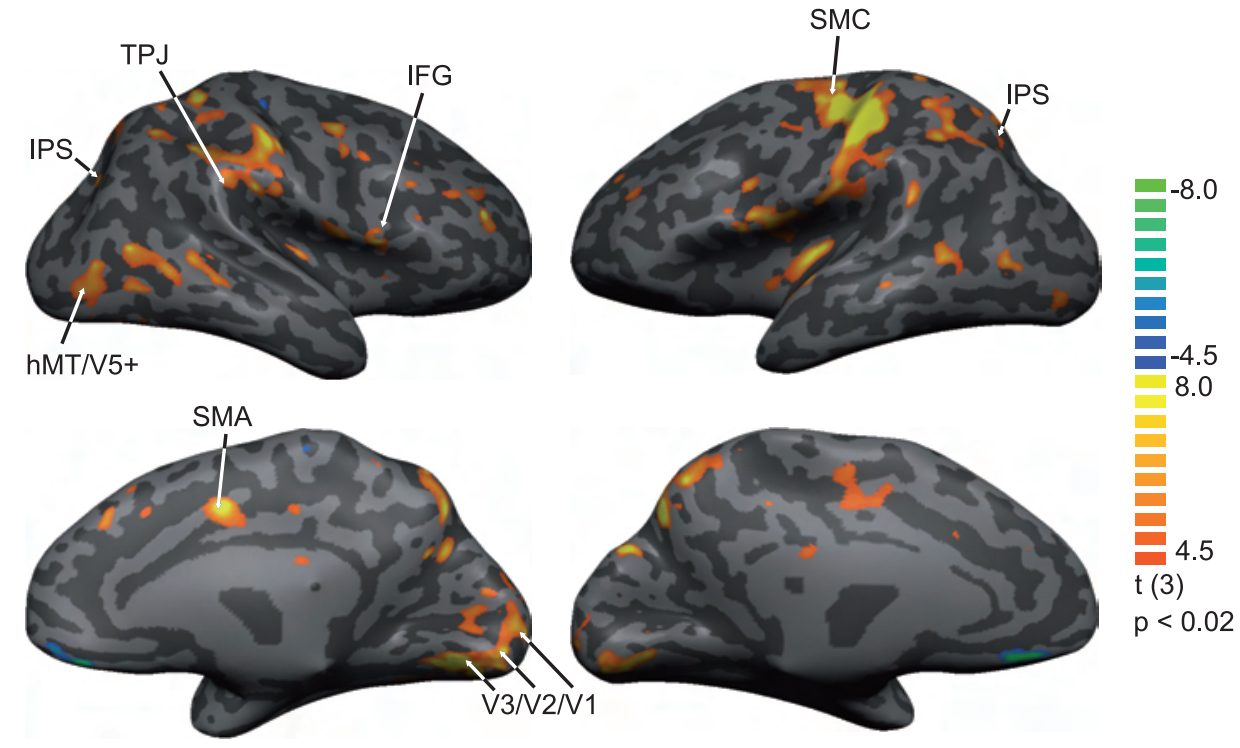

Figure 3. Group analysis. A random-effects group analysis was performed to identify areas that showed responses to switches between perceptual states. Data are displayed on the reconstructed and inflated hemispheres of participant AWH03. Top, lateral view of the right and left hemispheres. Bottom, medial view of the right and left hemispheres.
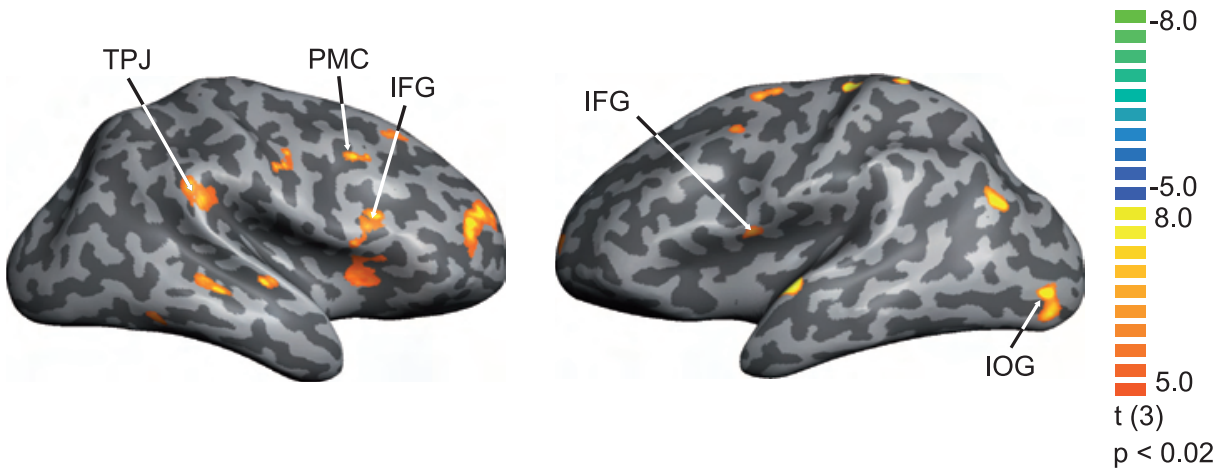

Figure 4. Multivoxel pattern analysis in lateral cortical areas. A searchlight-based multivariate approach was used to find areas in the brain that contained information about the perceived motion direction in the fine-grained pattern of local activity. Highly significant classification accuracy was found in bilateral inferior frontal gyrus (IFG), right temporoparietal junction (TPJ), right premotor cortex (PMC), and inferior occipital gyrus (IOG). 


\section{Discussion}

We used multivariate analysis techniques to find percept-related activity patterns in a network of cortical areas responding to switches of conscious motion perception. The stimulus consisted of a range of motion quartets, which induces a coherent perception of motion direction in all quartets. Such global perceptual effects are usually attributed to areas at intermediate and higher stages of the processing hierarchy. We did find very high levels of decoding performance in right IFG, right TPJ, and right PMC among other areas. But there were also clusters containing significant information about perceived motion direction in early areas.

The TPJ and IFG regions that we describe in our results might be part of a rightlateralized ventral attention network, which is involved in reorienting responses to behaviorally relevant stimuli (Corbetta and Shulman, 2002; Corbetta et al., 2000; 2008). Corbetta and Shulman (Corbetta and Shulman, 2002; Corbetta et al., 2008) differentiate between a bilateral dorsal attention network, including intraparietal sulcus, superior parietal lobe, and the frontal eye fields, and a ventral network with TPJ and IFG as core regions, but also including anterior insula, frontal operculum, and parts of middle frontal gyrus. The function of the dorsal network is maintaining attentional or task set over time in order to enhance processing of objects with specific task-defined features. The ventral network responds in a stimulus-driven manner to salient and unexpected events and is vital for reorienting to new information in the environment. In our multivariate analysis, all the subparts of the ventral attention network contained significant information about the perceived motion direction. The information cannot be derived from the motor response or sensorimotor transformations since the assignment of response buttons to perception was switched on every run. Therefore, the information must be closely related to the perceptual state of the observer.

Recently, it has been suggested that the right IFG is involved in the initiation of perceptual switches during ambiguous apparent motion (Sterzer et al., 2002). Our data show that percept-related content might be even represented in this frontal region. This suggests that IFG has not only the role to initiate perceptual switches nonspecifically, but that it contains information about the specific perceptual contents of conscious experience. It remains to be seen whether the area described by Sterzer and Kleinschmidt (2007) is overlapping with or separate from the ventral attentional network described above.

Interestingly, Corbetta and Shulman $(2002 ; 2008)$ relate the activation of the ventral attention network to noradrenergic modulation through the locus coeruleus (LC). The functions of the LC overlap largely with the ones described for the ventral attentional network and there is some anatomical evidence for connections between the two systems (Foote and Morrison, 1987; Morrison and Foote, 1986). Noradrenergic modulation is thought to be involved in stabilizing perceptual or cognitive decisions (Aston-Jones and Cohen, 2005), a function relating to perceptual switches in our bistable apparent-motion paradigm. In a previous study, LC activity was associated with perceptual switches in other bistable paradigms like binocular rivalry and structure-from-motion (Einhäuser et al., 2008). The authors measured pupil diameter as an indirect but reliable indicator of LC activity and could show that 1) pupil diameter increased just before reports of perceptual switches and 2) that pupil diameter could predict the duration 

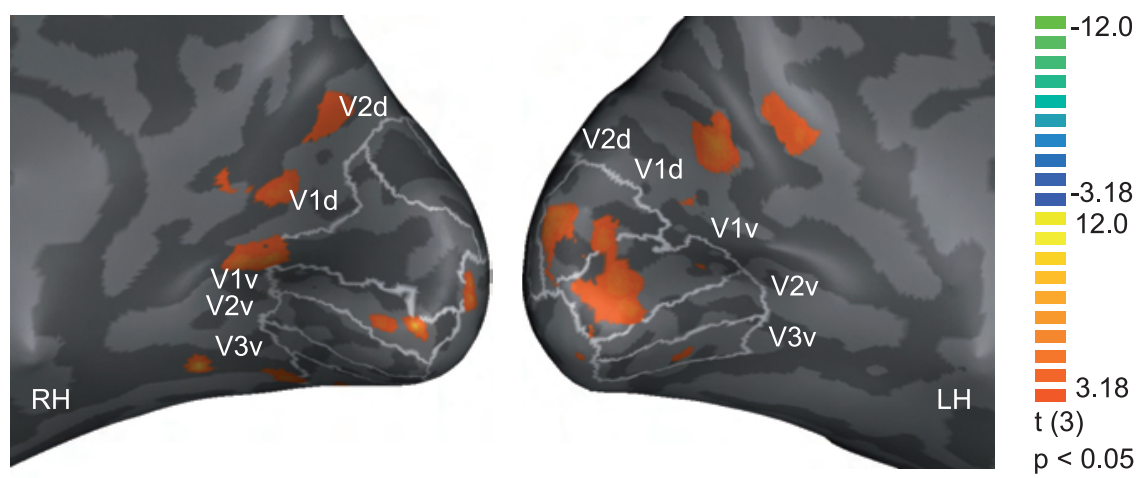

Figure 5. Multivoxel pattern analysis in early visual areas. Medial view on the two hemispheres focusing on early visual areas. Group data are displayed on the individual anatomy and retinotopy of participant AWH03. Although at a lower threshold $(p<0.05)$, early visual areas $V 1$, $\mathrm{V} 2$, and $\mathrm{V} 3$ contain also significant amounts of information about observers' current conscious percept. d, dorsal, RH, right hemisphere, LH, left hemisphere, v, ventral.

of the next perceptual phase. One possible interpretation of our results with respect to the studies described above is that the non-specific switch signal of LC neurons is translated into specific content already in the connected systems of the ventral attentional system. From there, other multi- and unimodal motion areas are updated even down to early visual areas.

Other studies have identified frontal and parietal areas related to the representation of motion in humans that are similar to regions found in our results. Bremmer and colleagues (2001) found large overlap in right ventral PMC, ventral intraparietal cortex, and lateral inferior postcentral cortex for processing of moving stimuli presented in the visual, auditory, and tactile modalities. Bremmer and colleagues could not rule out salience as a confounding factor in their study. But our results show that frontoparietal activity patterns contain specific information about the conscious perception of an observer, suggesting that those activations are not based on salience alone. Claeys et al. (2003) described a region in bilateral inferior parietal gyrus that is specifically involved in the processing of long-range apparent motion across the whole visual field. Claeys and colleagues used a motion quartet as stimulus, but they could only find significant responses for very fast apparent motion $(7 \mathrm{~Hz})$ compared to our parameters $(2.9 \mathrm{~Hz})$. Another difference is that we used a whole array of quartets in contrast to a single quartet. This might emphasize processing in higher-order motion regions in parietal cortex independent of the stimulation frequency used.

Several previous studies have shown that apparent motion produces activation in parts of $\mathrm{V} 1$ representing the path of illusory motion, where no physical stimulus is present (Larsen et al., 2006; Muckli et al., 2005; Sterzer et al., 2006). This activity is most likely generated by feedback connections from hMT/V5+ to V1. The retinotopic V1 activations were also investigated for the motion quartet and it could be shown 
that depending on perceived motion direction, the respective $\mathrm{V} 1$ representations of the vertical or horizontal motion path get activated (Muckli et al., 2005). The studies did not consider activity in V2 or V3 because smaller cortical size of these areas and the split between dorsal and ventral quarterfields render it more difficult to separate representations of apparent-motion inducers and the illusory path in those areas. Our study provides evidence that in addition to V1 differential retinotopic patterns of activity area might also be present in V2 and V3. It is unlikely that classification in early visual cortex is based on motion-sensitive subpopulations tuned for vertical and horizontal motion since the distance between inducers in our quartets was $5.71 \mathrm{deg}$. Receptivefield sizes in human visual areas V1-V3 are thought to be below 4 deg even at more eccentric visual-field locations (Smith et al., 2001).

The first study investigating the representation of motion direction in visual cortex (Kamitani and Tong, 2006) found high classification performance in early visual areas compared to later processing stages (especially hMT/V5+). But in this study, randomdot patterns were used, which are activating low-level motion sensors in primary areas. Two other studies employed motion stimuli in combination with multivariate analysis techniques. Brouwer and colleagues (2007) had participants report the perceived rotation direction in a moving structure-from-motion stimulus. In their group of observers, they could not decode reliably the perceived rotation direction from early visual cortex, but a significant amount of percept-related information was present in higher areas, i.e., V3A, V7, hV4d, and hMT/V5+, as well as IPS. Since their analysis was only performed in prespecified regions of interest, it is unclear whether the frontoparietal network we describe would show similar discriminative power for structure-from-motion.

A second study that used an ambiguous motion stimulus to find percept-related activity patterns in cortical areas was conducted by Serences and Boynton (2007). They used random-dot patterns with two different motion directions to find mean activation patterns in different areas representing the respective directions. In addition, they used a random-dot pattern with incoherent motion ( $0 \%$ coherence) and let observers report whether they saw one of the predefined motion directions. Although there was no bias in the stimulus towards one or the other direction, in most cases, participants reported one of the given directions after stimulus presentation. Despite the lack of coherent motion in the stimulus, Serences and Boynton were still able to classify the reported (and presumably perceived) direction from activity patterns in hMT/V5+. For all other areas (except maybe V3A), the predictive success declined to non-significant levels. Their result suggests that hMT/V5+ has a vital role for perceptual choices of motion direction.

A large number of previous fMRI studies have demonstrated the vital role of hMT/ V5+ for conscious motion perception using univariate (Goebel et al., 1998; Muckli et al., 2002; 2005; Sterzer and Kleinschmidt, 2005; 2007; Sterzer et al., 2002; 2003; 2006) and multivariate techniques (Brouwer and van Ee, 2007; Serences and Boynton, 2007). In comparison to previous studies using multivariate techniques to study motion perception, we found relatively low classification performance in and around hMT/V5+. It is possible that global long-range apparent-motion percepts as induced by our stimulus are more reliant on higher motion centers in parietal cortex, similar to the ones described by Claeys and colleagues (2003). 
In summary, we were able to decode information related to the subjectively experienced motion direction in an ambiguous motion display from early visual areas (V1-V3), as well as from parietal and frontal regions. The significant levels of performance in early areas can most likely be explained by feedback projections from higher areas generating distinct retinotopic patterns of activation for the different motion directions. Significant classification performance in parietal and even frontal areas suggests that information related to perceptual states might be represented to some degree in non-perceptual cortical regions. 



\section{Discussion}


The present chapter serves the main purpose of extending the discussions of the three research papers to put the findings into a broader context. In this respect, I will derive putative neuronal models for the different visual phenomena. Finally, a brief paragraph on conscious visual perception will close this dissertation.

\section{The cortical representation of objects rotating in depth}

In our study on apparent rotation described in Chapter 3 we identified several brain regions being involved in the processing of apparent rotation. Those regions were situated in the ventral as well as in the dorsal streams within the visual processing hierarchy (Goodale and Milner, 1992; Milner and Goodale, 2008; Ungerleider and Mishkin, 1982). Most importantly, the regions represented the rotating objects in a motion-specific, view-invariant fashion with respect to the motion path. In other words, those areas seem to encode views of a rotating 3D object (including illusory ones) that are linked by motion. In the discussion section of Chapter 2 we hypothesized that we found a 'cortical network' being involved in apparent rotation. We think that all those regions are not simply co-activated, but interact to form the basis for the illusory percept. In the following, I will concentrate on this hypothesis of a cortical network for apparent rotation. First, I try to match the individual areas that constitute the network with those found in related experiments. Second, I contrast our results with recent findings concerning the representation of objects in the dorsal stream (Konen and Kastner, 2008). Third, I evaluate interactions between ventral and dorsal stream areas. Finally, I propose a tentative model for apparent rotation and suggest future research experiments.

\section{The cortical network}

In our study we defined areas based on their anatomical localization since we did not perform additional functional localizer experiments. The network comprises regions in transverse occipital sulcus, lateral occipital sulcus, inferior right occipital gyrus, superior parietal cortex and occipito-temporal cortex. Matching these areas found in our study with areas identified by others is complicated by the fact that there is a vivid debate especially concerning the definition of higher visual areas (Malach et al., 2002; Wandell et al., 2005; 2007). Only few areas are agreed upon (early visual areas) and for the other ones there are numerous names. Thus, naturally, this matching procedure will be biased by my own 'philosophy' concerning those higher visual areas.

Based on Talairach coordinates, we identified the region in ventral cortex as a subregion of the LOC (Malach et al., 1995). LOC has been localized based on its higher activation profile for intact objects in contrast to scrambled objects (Kanwisher et al., 1996; Malach et al., 1995). In following studies, the large area was divided in subparts (Grill-Spector et al., 1999). LOC plays a major role in shape processing (Grill-Spector et al., 1998) with an emphasis on processing the perceived shape (Kourtzi and Kanwisher, $2000 ; 2001)$. LOC is particularly involved in the representation of perceived 3D-shapes (Kourtzi et al., 2003a; Moore and Engel, 2001; Welchman et al., 2005). In this respect, LOC is at an intermediate stage of object recognition; the final stages directly related to object recognition would be even more anterior regions of the inferior temporal cortex (Quiroga et al., 2008; Riesenhuber and Poggio, 1999; Tanaka, 2003). Most inter- 
estingly, LOC has been found to be involved in transformational apparent motion (Tse, 2006). With respect to retinotopically defined areas in ventral cortex, the ventral/lateral region of our study might partly overlap with LO-1 and LO-2 and even VO-2 (Brewer et al., 2005; Larsson and Heeger, 2006; Wandell et al., 2005; 2007).

We identified several areas in the dorsal processing stream from the inferior occipital gyrus over regions in the intraparietal sulcus to superior parietal cortex. Based on visual inspection and comparison of Talairach coordinates, those areas might partly correspond to V3A/B and IPS-0 (V7), IPS-1 and maybe even IPS-2 (Levy et al., 2007; Swisher et al., 2007; Wandell et al., 2007). Apart from their retinotopic organization, those areas have been identified in humans in studies on motion processing (V3A; Tootell et al., 1997; Goebel et al., 1998; V3B, Smith et al., 1998), spatial attention (IPS-0/V7; Tootell et al., 1998b), saccade planning and visually-guided hand movements (IPS-1 and IPS-2; Hagler et al., 2007; Levy et al., 2007). A recent study demonstrated that all those areas can also be identified purely by using sensory stimulation (Swisher et al., 2007). It has been shown that V3A responds differentially to various types of global motion, such as radial and translational motion (Koyama et al., 2005). To our knowledge there is no study yet on the locus of highest sensitivity for in-depth rotational motion. However, Yang et al. (2006) have shown that responses to stimuli causing motion in depth seem to be allocated more to area V3A than to hMT+/V5. We did not observe area hMT+/ V5 in our study on apparent rotation. This is at a first glance surprising given the fact that the area is crucial for the phenomenon of apparent motion (Goebel et al., 1998; Muckli et al., 2002; 2005). However, our fMRI-adaptation approach specifically aimed at localizing regions that represent the illusory rotation object. We did not identify all regions involved in the broader processing of apparent rotation per se. That is, if we had contrasted an apparent rotation sequence with a flickering object or even with a static display, we most certainly would have found hMT+/V5 (and also other areas). Concerning the regions in the IPS, all aforementioned roles such as in spatial attention, eye movement control, and grasping might play an important part in apparent rotation. Spatial attention is of course needed to attentively track objects (Culham et al., 1998; Xu and Chun, 2006). As we have seen in our study, if attention is drawn away from the objects to a demanding center task, the established network does no longer represent the illusory rotating object. In our study we successfully prevented subjects from making eye movements to the rotating objects, in real life situations, however, rotating objects of course provoke smooth pursuit eye movements as well as saccades.

All those regions show view-invariant representations of the illusory object that are motion-specific (Stone, 1999). Thus, despite their potentially different functional roles in the processing of apparent rotation, those areas of the ventral and dorsal stream finally represent the illusory object. Concerning the region in ventral visual cortex or LOC, this finding is not surprising. Studies on viewpoint effects in ventral areas have found a gradient from viewpoint-dependent object representations in more posterior regions to some degree of viewpoint-invariant object representations in ventral regions (James et al., 2002). However, the issue of specific object representations in parietal cortex is of a much more controversial nature (Grill-Spector, 2003; Murray et al., 2003; Sereno et al., 2001). 


\section{Object representations in the dorsal stream}

A recent study by Konen and Kastner (2008) found viewpoint-dependent object representations in intermediate areas (V3A, MT and V7/IPS0) and viewpoint-invariant object representations in higher-level areas (IPS1, IPS2) of the dorsal stream. Previous fMRI studies have revealed view-dependent object representations in dorsal areas of the visual processing hierarchy (James et al., 2002; Vuilleumier et al., 2002). Taking a closer look at the reported Talairach coordinates, the region in dorsal cortex found by Vuilleumier et al. (2002) seems to be related to the intermediate areas (V3A, V7/IPS0) defined by Konen and Kastner (2008). The region found by James et al. (2002) seems to be closer to area IPS1 as defined by Konen and Kastner (2008). Thus, the observed viewpoint-invariant object recognition in higher-level areas of the IPS is a new finding and is not contradictory to the previous studies. However, concerning the role of especially the more anterior regions of the IPS in visually guided hand movements (Grefkes and Fink, 2005), the result on the view-invariant object representation of Konen and Kastner (2008) is still surprising. In order to grasp a particular object, the system should retain the specific information concerning the appearance of the object and not generalize across different viewpoints (Jeannerod et al., 1995). Thus, the functional role of object representations in dorsal areas is still unclear.

All aforementioned studies displayed static objects. But, as we already pointed out in Chapters 2 and 3, changes in viewpoint naturally occur because either the object or the observer is moving. (For a general discussion on the impact of object motion on object recognition see Vuong and Tarr, 2004). Furthermore, areas of the dorsal stream receive immediate input from motion processing regions - if they are not directly involved in motion processing. Thus, by employing (apparent) motion, we are able to put object representations in the dorsal system to a straight test. We reveal viewpoint-invariant object representations of the rotating object with respect to the rotation path. In other words, those views of the object that lay on the rotation path are represented in areas of the dorsal path. Views outside the path are not represented. Most importantly, intermediate as well as higher-level areas demonstrated such a motion-specific, view-invariant object representation.

A constrained representation of object views that are linked by motion enables the dorsal system to support precisely adjusted movements towards rotating objects. If the object is moving, while the observer tries to grasp it, this requires an additional online computation of motion and shape information in combination. One might speculate, however, that the system should also represent the extrapolated object views in accordance with the direction of motion (Mitsumatsu and Yokosawa, 2003; Munger et al., 1999). Thereby, the dorsal path would provide a predictive signal for motor areas to accommodate reaching to moving objects. This would be especially helpful for faster moving objects. In our study there was only one motion sweep, and the direction of motion in a single trial was unpredictable. Thus, I think that the extrapolated versions were not represented because subjects did not expect the object to move further.

\section{Interaction between the dorsal and ventral pathway}

Based on the different profiles of intermediate versus higher-level visual areas in ventral as well as in dorsal pathways, Konen and Kastner (2008) interpret their findings as evidence for two parallel and hierarchically organized neural systems for object 
representation along the ventral and dorsal visual pathways. They argue against the two-pathway hypotheses and for parallel encoding of object information in the two pathways. We show that if motion comes into play, higher-visual areas as well as intermediate areas now display the same functional profile, representing those views that are linked by motion. Contrary to the hypothesis of Konen and Kastner (2008) I believe that interactions between areas of the ventral and dorsal stream underlie the perception of apparent rotation and consequently the formation of object representations in those regions.

There is strong empirical evidence for a tight coupling between the two visual processing streams at all levels of processing (e.g. Felleman and Van Essen, 1991; Merigan and Maunsell, 1993; Peuskens et al., 2004). Cross-connections between macaque areas V4 and MT, for example, have been intensively studied (Chen et al., 2007; Maunsell and Van Essen, 1983; Schroeder et al., 1998; Stepniewska et al., 2005; Ungerleider and Desimone, 1986). The two areas are reciprocally connected (Maunsell and Van Essen, 1983; Ungerleider and Desimone, 1986). Studying response latencies, Schroeder et al. (1998) found markedly different latencies for areas in the ventral and dorsal stream and suggest a temporal ordering of visual responses. More precisely, they found evidence for input from the 'fast visual system' (MT, STS) into the 'slow visual system' (V4, IT; Chen et al., 2007).Thereby, motion-sensitive inputs into the ventral stream might aid e.g. figureground segregation (Ferrera et al., 1994). A recent study employed simultaneous EEG and MEG recordings (Jiang et al., 2008). They presented either static objects or objects defined by structure-from-motion and analyzed the sequence of cortical activation. For static displays, the sequence of activation was: early visual cortex $>$ lateral occipital regions > ventral temporal regions > parietal cortex and again early visual cortex. In contrast, for the SFM stimuli, the sequence of activation was: early visual cortex $>$ middle occipital regions $>$ parietal cortex $>$ lateral occipital cortex $>$ ventral temporal regions $>$ again parietal cortex, early visual cortex and lateral occipital cortex (Jiang et al., 2008). Thus, this study further confirms the input of motion-sensitive areas into ventral visual areas, if moving stimuli are observed. Furthermore, Jiang and coworkers (2008) demonstrate a differential functional profile of the parietal regions: they are involved in an early and a late phase with moving objects, but only in a late phase with static objects.

\section{A putative model for apparent rotation}

Based on all these considerations I put forward a putative model for apparent rotation. Figure 1 depicts a schematic illustration of the model. The model involves areas of the dorsal stream (V3A, hMT+) extracting the (apparent) motion information and feeding this information into ventral regions. LOC conveys the shape information needed to perceive the 3D object. Reciprocal connections between dorsal and ventral areas and the special role of superior parietal cortex enable the online tracking of the (apparently) rotating object. Because of exchange between regions, object representations that are confined to the perceived rotating views of the object are formed both in ventral as well as in dorsal areas. Finally, frontal areas are involved in task-related computations that lead to the motor output according to the task. Similar models have been proposed for the highly related phenomenon of structure-from-motion (Kriegeskorte et al., 2003; Murray et al., 2003; Orban et al., 1999; Paradis et al., 2000). An open 


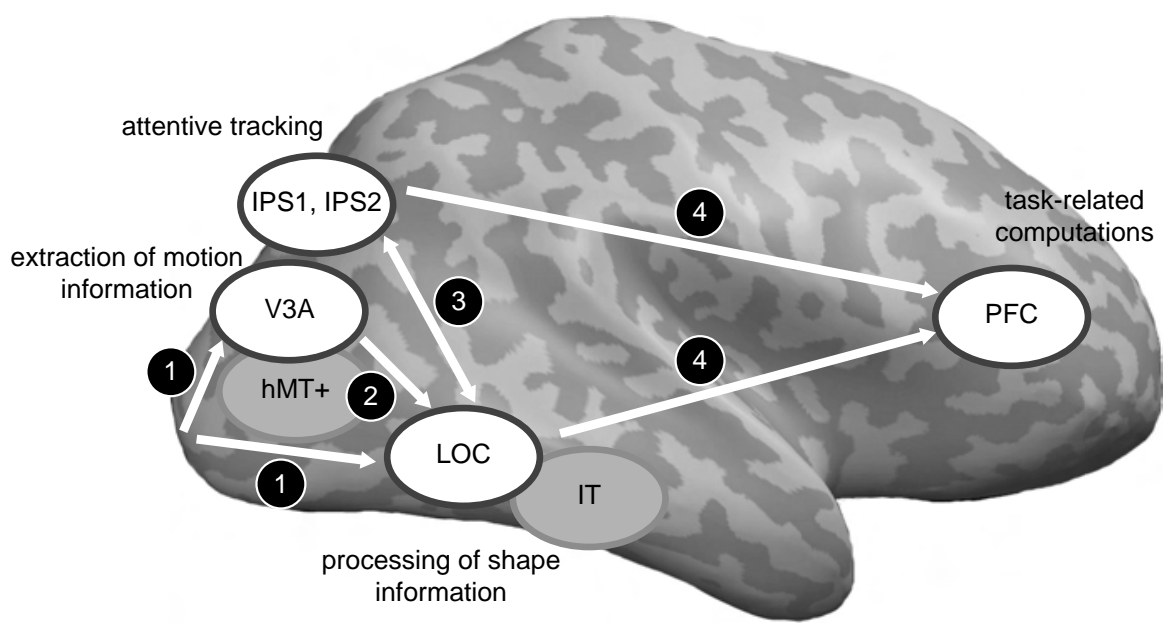

Figure 1. A putative model for apparent rotation. Early visual cortices project to the dorsal and ventral processing stream. Intermediate dorsal stream areas such as V3A, hMT+ extract the motion information. Via fast connections this information is fed into ventral stream areas such as LOC. The LOC processes the shape information. Reciprocal connections between dorsal and ventral visuals and the special role of superior parietal cortex enable the online tracking of rotating objects. Object representations of interpolated rotations views are thus represented both in ventral and dorsal processing pathways (white ovals). Specific frontal cortices are involved in task-related computations.

question is of course where and when and maybe even if at all in this network the conscious perception of apparent rotation 'emerges'. I will discuss this issue briefly in the last paragraph of this discussion. Of course this model needs further empirical testing. Future research on apparent rotation might focus on the interactions within the cortical network employing connectivity measurements. Moreover, the role of object representations in dorsal areas needs further clarification. The differences between our study and the study by Konen and Kastner (2008) might be mainly attributable to the different stimuli (static vs. moving). However, they might also be due to different experimental approaches. As we have reviewed in Chapter 2, fMRI-adaptation effects are highly susceptible to different experimental parameters. Thus, to address the question concerning object representations in dorsal areas conclusively, one might have to conceive a $2 \times 2 \times 2$-factorial design with type of stimulus (static vs moving), viewpoint (same vs different) and experimental approach (event-related vs block design). 


\section{Separate cortical stages in amodal completion}

In our study on amodal completion described in Chapter 4 we found that regions of the inferior temporal cortex (ITC) represent the amodally completed shape. ITC adapts to the amodally completed shape as if a whole shape had been presented. In contrast, early visual cortex (V1) represents the physically presented stimulus. We interpret our results as evidence for a multiple-step theory of amodal completion (Sekuler and Palmer, 1992), starting with a representation of the local contour information in V1 and ending with a representation of the completed shape in ITC. Our study was not devised to identify the actual computations underlying amodal completion in-between V1 and ITC. Nevertheless, we speculate that a feedforward model might explain the process of amodal completion.

In the following, I will further discuss possible neuronal models underlying amodal as well as modal completion. Starting with amodal completion, I review the neuroscientific literature and evaluate two opposing models. I will then switch the topic to modal completion, again summing up neuroscientific findings and deriving alternative models. Finally, I will discuss similarities and differences between amodal and modal completion in terms of their psychological as well as neuroscientific basis. Our aim is to provide an integrative neuronal model for both amodal and modal completion.

\section{Neurophysiological studies on amodal completion}

Only few studies using single-cell recordings (Bakin et al., 2000; Lee and Nguyen, 2001; Sugita, 1999), fMRI (Kourtzi and Kanwisher, 2001; Lerner et al., 2002; Rauschenberger et al., 2006) and MEG (de Wit et al., 2007; Liu et al., 2006; Murray et al., 2004; Plomp et al., 2006) have been conducted to unravel the neurophysiological basis of amodal completion (in comparison to modal completion). There are two main research questions: Of course, the most important question is where the amodally completed shape is represented? Or in other words, which brain regions signal the completed shape? The second question concerns the so-called mosaic stage in amodal completion, i.e. the representation of the actual physical properties of the stimulus. Is there such a mosaic stage in amodal completion and if so, which brain areas do represent it?

Electrophysiological studies focused on early visual areas. Bakin and colleagues (2000) and Lee and Nguyen (2001) found neuronal responses to amodally completed shapes as early as V1 and V2. Responses in V1 were always weaker than responses in V2. Furthermore, the response latencies were longer in V1 as compared to V2 and always delayed in comparison to real contours (Lee and Nguyen, 2001). In contrast, Sugita (1999) only investigated V1 and found responses to amodally completed bars with the same latency as for real bars. Neuroimaging studies concentrated on higher visual areas and found evidence for the involvement of the lateral occipital complex (LOC) in amodal completion (Kourtzi and Kanwisher, 2001; Lerner et al., 2002). In accordance, MEG experiments found a processing component reflecting amodal completion that has been located in higher-level visual areas (Liu et al., 2006; Plomp et al., 2006). Interestingly, those MEG studies also revealed an early processing component possibly reflecting the mosaic stage (Liu et al., 2006; Plomp et al., 2006).

There is no single-cell study on amodal completion that tested higher-visual areas, as there is no neuroimaging study that displayed early visual areas representing 
amodally completed shapes. Thus, there is evidence for the participation of early visual areas in amodal completion by electrophysiological studies and for the involvement of later visual areas by neuroimaging techniques. In order to derive a neuronal model for amodal completion one would need to know the exact role of the single areas as well as the timing of the different processing steps. However, there are some hints that point towards two putative models for amodal completion.

\section{Feedforward models of amodal completion}

A serial processing model of amodal completion was suggested by Sekuler and Palmer (1992) and Rauschenberger and Yantis (2001). The neuronal feedforward model is based on the general feedforward sweep from primary visual cortex to area V2 and V4 and further along the ventral processing stream (Felleman and Van Essen, 1991). In that model (see Figure 2), V1 represents the mosaic stage of amodal completion, extracting the actual contours present in the image. V2 serves a most important role, namely resolving the problem of border ownership (Bakin et al., 2000; Qiu and Von der Heydt, 2005; Zhou et al., 2000) and the more general function of grouping contour features for the purpose of object identification (Qui and Von der Heydt, 2007). Real contours are assigned to different objects and missing contours are filled in on the basis of Gestalt rules. This last step might take place in interaction with V4. The fully completed object is represented in the LOC. Our own study and the other neuroimaging (Kourtzi and Kanwisher, 2001; Lerner et al., 2002) and MEG studies (Liu et al., 2006; Plomp et al., 2006) can be interpreted in favor of this model. The critical involvement of $\mathrm{V} 2$ that was found by electrophysiologists is also in congruence with this model. However, the responses of V1 (Lee and Nguyen, 2001; Sugita, 1999) do not fit with this model. Based on the latency shift between V2 and V1 observed by Lee and Nguyen (2001) one might suggest that the observed response in $\mathrm{V} 1$ is based on a feedback signal, presumably from area V2. The data of Sugita (1999) however, stands in direct contrast with this.

\section{Feedback models of amodal completion}

A feedback model of amodal completion was proposed by Murray and co-workers (2004) based on their studies on amodal as well as modal completion. Using EEG, Murray et al. (2004) found a first component at 140-238 ms that they located in higherlevel visual areas (posterior parietal as well as lateral occipital regions) using source localization. Furthermore, amodal completion evoked continued strong responses in these structures also in a subsequent time period (240-298 ms). Murray et al. (2004) proposed that amodal completion is initiated in higher-level visual areas and that the responses seen by electrophysiologists are due to feedback modulations (Figure 3). An alternative interpretation might be that the process of amodal completion is initiated earlier, presumably in V2, and that the process regarding the representation of the fully completed shape in the higher-visual areas dominates the signal. The assumption of a feedback signal to early visual areas was derived from their earlier study on modal completion (Murray et al., 2002). However, it is still under debate whether the processes of modal and amodal completion are fully overlapping, and Murray et al. (2004) demonstrated that the two phenomena differ during a late time window. Furthermore, this theory does not provide an explanation how amodal completion is established in the higher-level visual areas and thus clearly needs further exploration. 


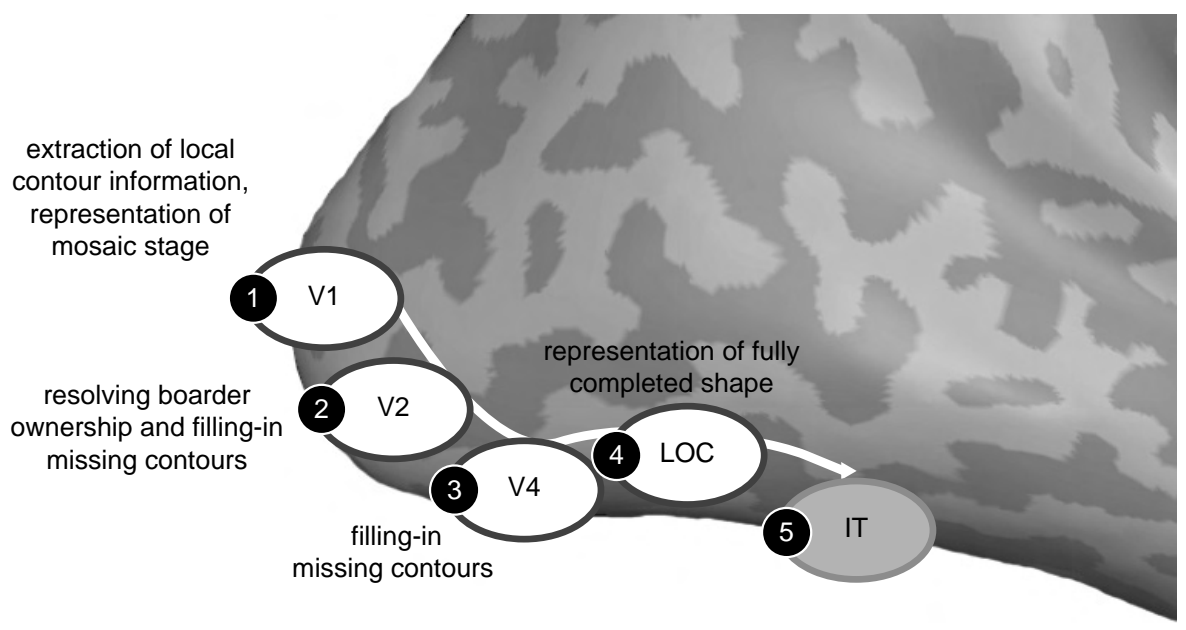

Figure 2. A feedforward model for amodal completion. $V 1$ extracts the local contour information and thus represents the mosaic stage. V2 resolves the border-ownernship problem by assigning borders to defined objects and filling-in missing contours. Further along the ventral processing stream, V4 aids in filling-in missing contours and finally the fully completed shape is represented in higher-level visual area LOC.

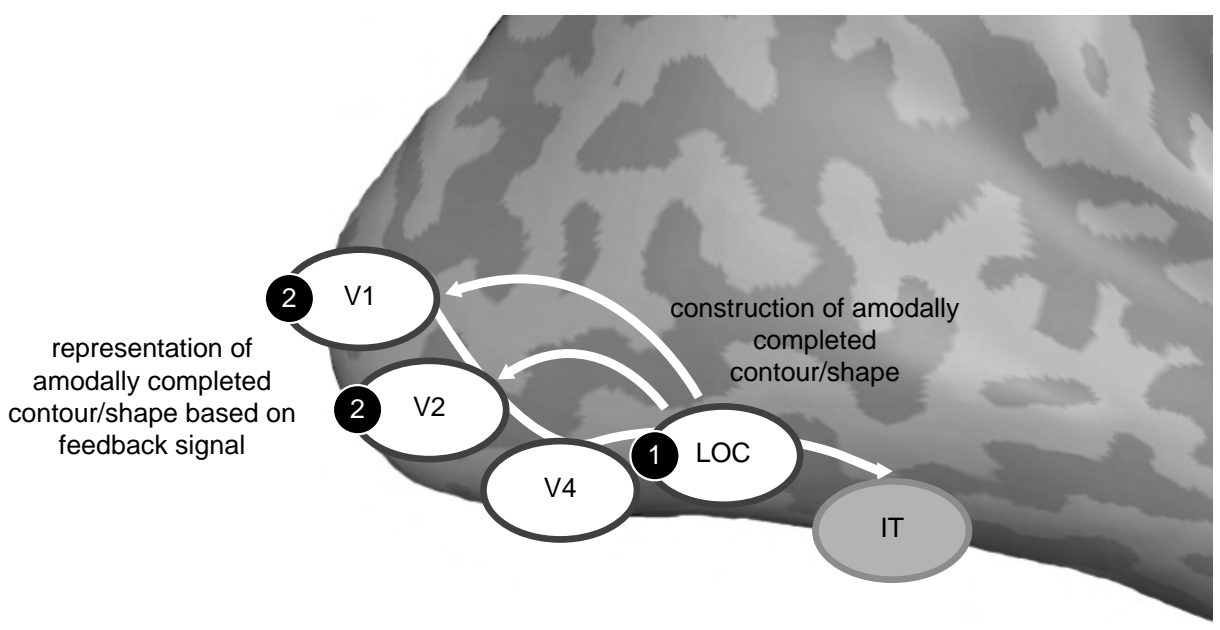

Figure 3. A feedback model for amodal completion. Processing starts with a coarse representation of the amodally completed shape in higher-level visual area LOC. LOC projects feedback signals to early visual areas that represent the amodally completed contour. 
In summary, two alternative models might account for the process of amodal completion. No direct test of these models, e.g. employing TMS, has yet been performed. However, similar models have been proposed for modal completion. Evaluating the empirical evidence for these models of modal completion might also shed light on a putative model for amodal completion.

\section{Neurophysiological studies on modal completion}

The vast majority of studies on perceptual completion phenomena deal with modal completion or illusory contours. This is remarkable since amodal completion is far more common in our natural environment than modal completion (Tse, 1999). Neurophysiological studies on modal completion have been recently reviewed by Seghier and Vuilleumier (2006). The major research question behind those studies on modal completion is the same as for the studies on amodal completion: where in the brain are illusory contours represented? Which brain processes are involved in the computations leading to an illusory percept? Interestingly, in contrast to the studies of amodal completion, the question concerning the inducing stimulus and its representation in the brain were not of interest. However, I would like to emphasize that one of the typical stimuli used to elicit illusory contour perception, namely the Kanisza triangle or square, does involve amodal completion (Wagemans et al., 2006). If the inducing 'pacmen' were not perceived as completed circles being occluded by a white triangle or square, the illusion wouldn't work. Another stimulus frequently used that does not have this problem is the abutting-line grating illusion (e.g. Montaser-Kouhsari et al., 2007).

Electrophysiological studies on modal completion that were conducted in cats and macaque monkeys focused again mainly on early visual areas (Grosof et al., 1993; Lee and Nguyen, 2001; Leventhal et al., 1998; Peterhans and von der Heydt, 1989; Ramsden et al., 2001; Redies et al., 1986; Sheth et al., 1996; von der Heydt and Peterhans, 1989; von der Heydt et al., 1984). Reliable responses to illusory contours were found in area V2 in almost all studies (Lee and Nguyen, 2001; Leventhal et al., 1998; Peterhans and von der Heydt, 1989; Ramsden et al., 2001; Sheth et al., 1996; von der Heydt and Peterhans, 1989; von der Heydt et al., 1984). In contrast, responses of area V1 were only sometimes observed and if so, they tended to be weaker than the responses of area V2 (Grosof et al., 1993; Lee and Nguyen, 2001; Leventhal et al., 1998; Redies et al., 1986; Sheth et al., 1996). Von der Heydt and colleagues (Peterhans and von der Heydt, 1989; von der Heydt and Peterhans, 1989; von der Heydt et al., 1984) did not observe effects in V1 despite clear evidence for illusory-related activity in V2. There is also a study on higher-level areas, namely IT cortex: Kovacs et al. (1995) found that units in IT cortex respond to whole, partial and occluded shapes and that they reduce their responses when the proportion of occlusion is increased.

Neuroimaging studies have revealed evidence for processing related to illusory perception in all visual areas from V1 up to ITC (ffytche and Zeki, 1996; Larsson et al., 1999; Maertens and Pollmann, 2005; Mendola et al., 1999; Montaser-Kouhsari et al., 2007; Stanley and Rubin, 2003). The study by Montaser-Kouhsari and colleagues (2007) is especially interesting, because they show orientation-selective fMRI adaptation to illusory contours. Thus, beyond stating that the visual areas are somehow involved in processing of illusory contours, they were able to demonstrate that the representa- 
tion is orientation-selective. Still, this study does not solve the question which areas are critical for the perception of an illusory contour (Weigelt, 2007). These studies are complemented by experiments on illusory motion that displayed responses in primary visual cortex to moving illusory contours (Seghier et al., 2000) or squares (Muckli et al., 2005).

EEG studies have demonstrated VEP modulations that were observable over lateral occipital electrodes. Those modulations were found at 88-100 ms (Murray et al., 2002), 126-186 (Murray, Imber et al., 2006), 140-238 ms (Murray et al., 2004) or $170 \mathrm{~ms}$ (Kruggel et al., 2001). Halgren and coworkers (2003) showed a component at 110 ms over the occipital pole and a later component ( $155 \mathrm{~ms}$ ) over lateral occipital cortex. Even later in time this activity seemed to spread back to the occipital pole as well as further along ventral cortex, finally reaching orbito-frontal cortex. Yoshino et al. (2006) found an ERP component 70-180 ms over lateral occipital cortex and a later 170-180 ms component over the occipital pole. Ablation experiments have shown that lesions to early visual areas (de Weerd et al., 1993), area V4 (de Weerd et al., 1996) and ITC (Huxlin et al., 2000) lead to deficits in the discrimination of illusory contours.

In summary, there is evidence for the involvement of early as well as intermediate and late visual areas in modal completion. The involvement of early visual areas seems to be more pronounced in modal completion as compared to amodal completion. Again, V2 seems to serve a critical purpose since activity in this region has been reliably associated with illusory contour perception in all electrophysiological studies. EEG and MEG experiments point towards possible feedback signals from higher-visual areas to lower visual areas. The ablation experiments show that modal completion can be disturbed at various levels of the processing hierarchy.

As for amodal completion different models can be derived from this that divide into feedforward and feedback models. Please note that this distinction does not imply that these models are either purely feedback or purely feedforward.

\section{Feedforward models of modal completion}

One bottom-up approach was proposed by Pillow and Rubin (2002). They suggest a cascade-like mechanism in early visual cortex as the underlying process for modal completion. In this model, illusory contours are generated via a cascade or chain of activity passed between neighboring, small receptive-field units (Figure 4). Such a process is likely to occur in early visual cortex (V1/V2) and the signal is then fed forward along ventral visual cortex. Similar models have also been put forward by von der Heydt and co-workers (Peterhans and von der Heydt, 1989; von der Heydt and Peterhans, 1989; von der Heydt et al., 1984) with a special emphasis on the role of area V2 and also by Lee and Nguyen (2001). Von der Heydt et al. argue that besides a slow 'edge-detection path' a faster 'grouping path' involving area V2 might aid contour completion. In both cases - V1 and/or V2 - lateral horizontal connections might serve the purpose of contour completion (Gilbert and Wiesel, 1992). Pillow and Rubin (2002) argued for an important role of early, retinotopic areas based on their finding that modal completion across the vertical meridian is much poorer than within one hemifield. This specific across-meridian impairment seems to bear on a higher sensitivity of early areas to interhemispheric divide. However, the authors also emphasize that feedback from higher visual areas might manipulate or even launch the cascade process. Maertens 


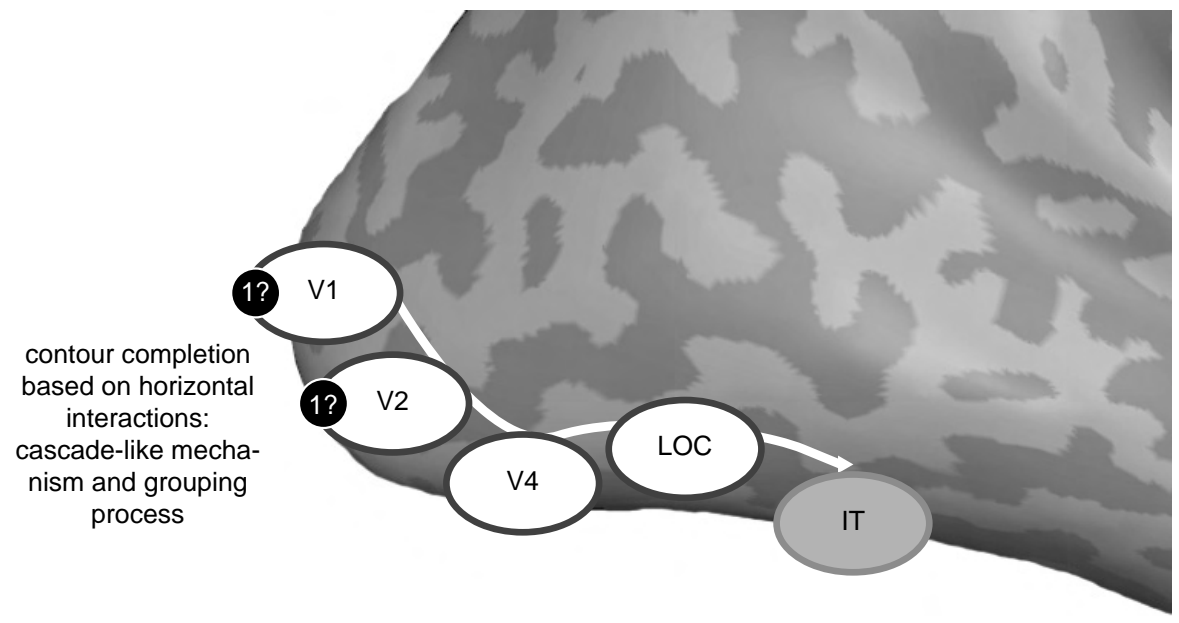

Figure 4. A feedforward model for modal completion. V1 or V2 compute contour completion. The completion is based on a cascade-like mechanism and grouping processes that are based on fast horizontal interactions within the areas. The signal of an illusory contour is passed further along the ventral processing stream.

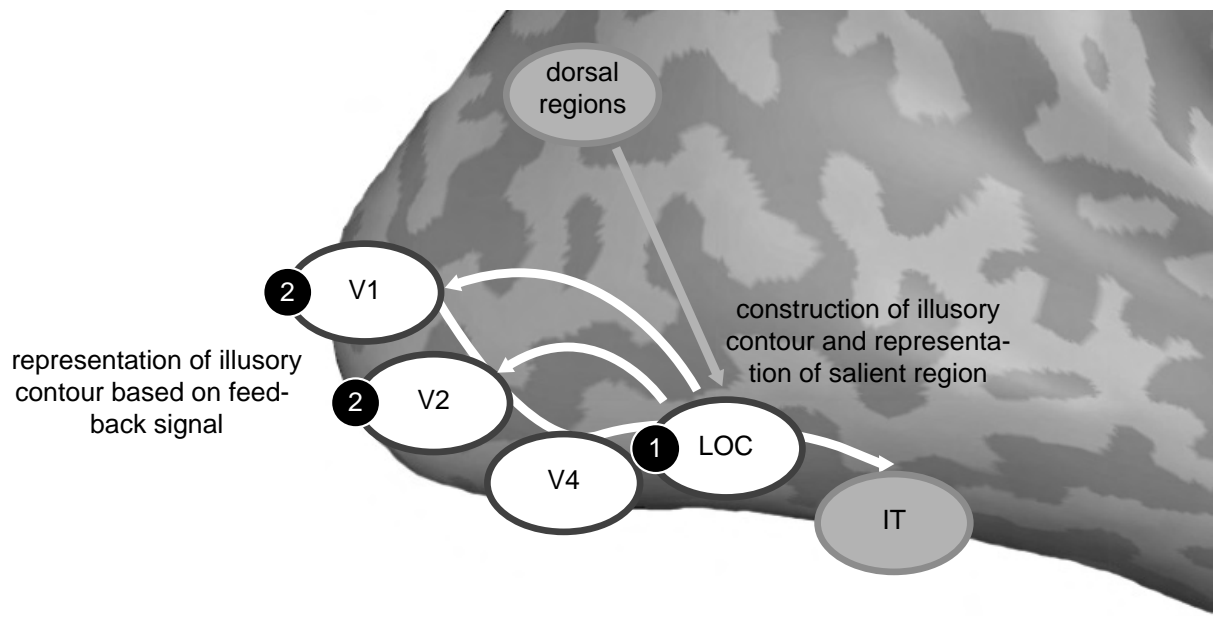

Figure 5. A feedback model for modal completion. LOC constructs and the illusory contour represents the salient region. $L O C$ sends feedback connections to early visual areas, where further computations that finally lead to the representation of the illusory contour are initiated. Murray et al. (2004) even suggest that the process starts within dorsal regions with a coarse object representation that is signaled to $L O C$ and that guides processing in ventral visual areas. 
and Pollmann (2005) observed a BOLD signal increase in V1 and a decrease in LOC after subjects were intensively trained to discriminate illusory contours. The authors interpret their findings as evidence for the important role of primary visual cortex in contour integration.

\section{Feedback models of modal completion}

The feedback model of modal completion starts with illusory-related processing in area LOC. LOC then sends feedback signals to early visual areas V1/V2 (Figure 5). In this model the computations underlying illusory contours occur in higher-visual areas and the observed responses in early visual areas merely reflect a feedback signal. Such a model is supported by the study of Yoshino et al. (2006) and also favored by Maertens and Pollmann (2007) as well as Stanley and Rubin (2004). Murray and coworkers (2002) also put forward such a model. However, they suggest that dorsal areas are involved even earlier than LOC, mediating configural information that guides the LOC. Yoshino et al. (2006) presented intriguing insights into the potential role of the feedback to early visual areas. Their investigation was based on the findings of Stanley and Rubin (2004) who demonstrated that a modification of the pacmen inducers led to a disappearance of the illusory contours while subjects still perceived 'salient regions'. Yoshino and co-workers (2006) demonstrated that the perception of illusory contours and that of salient regions share an early component over lateral occipital cortex (70-180 ms). However, only if illusory contours are perceived, a later component (170-180 ms) over the occipital pole can be observed.Thus, the later component might reflect a feedback from higher-level visual areas specific to (illusory) contours. It should be noted however, that the study by Yoshino et al. (2006) is the only direct empirical evidence for a feedback signal from LOC to V1/V2 in the course of modal completion. Other researchers that favor this model (Maertens and Pollmann, 2007; Murray et al., 2002; 2004; Stanley and Rubin, 2004) assume that the responses observed in V1 and V2 might be due to feedback but do not have direct evidence for such a claim.

In summary, as in amodal completion two alternative models might account for the process of modal completion. And as in amodal completion, no direct test of these models has yet been performed. In the following paragraph I speculate on a putative combined model for amodal and modal completion.

\section{A combined model for amodal and modal completion}

It is fiercely debated in the realm of psychology whether amodal and modal completion rely on common mechanisms (Anderson et al., 2002; Anderson, 2007a, b; Kellman et al., 2007; Singh, 2004). I feel that I am not able to take a firm stand in this debate. However I still want to propose a putative combined model for amodal and modal completion based on the neuroscientific evidence (Figure 6). This model consists of common as well as distinct neuronal mechanisms for modal and amodal completion.

One of the main ideas behind a combined model is that I assume highly similar processes for the contour completion mechanisms (at least in terms of the neuronal substrate underlying these processes). Both, in amodal as well as in modal completion, area V2 seems to serve an important role, since almost all studies found reliable responses in area $\mathrm{V} 2$ for both cases of perceptual completion (amodal completion: Bakin et al.,2000; Lee and Nguyen, 2001; modal completion:Lee and Nguyen, 2001; Leventhal 
et al., 1998; Peterhans and von der Heydt, 1989; Ramsden et al., 2001; Sheth et al., 1996; von der Heydt and Peterhans, 1989; von der Heydt et al., 1984). I assume that in area V2 major computations concerning the contour completion mechanism take place. These computations might involve the signaling of border ownership (Bakin et al., 2000; Qiu and Von der Heydt, 2005; Zhou et al., 2000) as well as end stopping (Peterhans and von der Heydt, 1993). In favor of an early involvement of area V2 is the study by Lee and Nguyen (2001) that found very short latencies in this area.

Within our putative model, V2 passes its signals via V4 to higher-level visual area LOC.Vinberg and Grill-Spector (2008) have demonstrated that LOC actually processes shapes, neither edges nor surfaces. LOC is sensitive to border ownership (Vinberg and Grill-Spector, 2008), which speaks in favor of major input from V2. The intermediate area V4 might be involved in transformations of contour-related signals to shape-related signals (Fulvio and Singh, 2006). V4 has been identified to compute curvatures and thus represents shapes at least partly in terms of the configurations and positions of their contour components (Pasupathy, 2006; Pasupathy and Connor, 2001). In the end, LOC might represent amodally completed shapes or objects as well as salient regions.

Up to this point, amodal and modal completion might rely on similar neuronal processing. It is possible that the way V2 resolves the contour completion is different for amodal as compared to modal completion. However, I suggest a further distinction between the two phenomena that is related to feedback connections to primary visual cortex. I suggest that in the case of modal completion, that is, in the case of an illusory contour and shape, feedback connections from the higher-visual areas to early visual areas are necessary to bring about the illusory percept. In contrast, in the case of amodal completion, there is no strong conscious percept of an existing contour or shape. As already pointed out by Michotte (1964), the two phenomena are in this respect phenomenologically clearly distinct. I thus agree with the above proposed models that the responses in V1 might reflect feedback activity. However in extension to the above described model I assume the feedback in the case of modal completion to be much stronger or even to be of a qualitatively different form than that in the case of amodal completion. Supporting this notion is the fact that responses in V1 are more reliably found and much stronger for modal than for amodal completion (Lee and Nguyen, 2001). Thus, the strength of feedback input to V1 might correlate with the perceptual strength of the phenomenon (Boynton et al., 1999; Polonsky et al., 2000).

In this respect, I think it is useful to compare both amodal and modal completion to related visual phenomena. Modal completion is highly related to apparent motion and filling-in phenomena. In all cases, illusory percepts are generated. It has been shown that apparent motion (Larsen et al., 2006; Muckli et al., 2005) as well as filling-in (Komatsu, 2006; Meng et al., 2005; Sasaki and Watanabe,2004) produces activity in primary visual cortex that is most likely due to feedback activity. In contrast, amodal completion is more related to perceptual closure phenomena and these have been shown to activate higher-level visual areas only (Gerlach et al., 2002; Sehatpour et al., 2006).

As an extension to the above described feedback models, I also want to emphasize that activity in V1 might also be at least partly derived from fast feedback from area V2. Feedback connections from V2 to V1 have been suggested to play a role in enhancing the responses in V1 to collinear contour elements (Shmuel et al., 2005). It has also been shown that contour-related responses in V1 strongly depend on top-down influences 


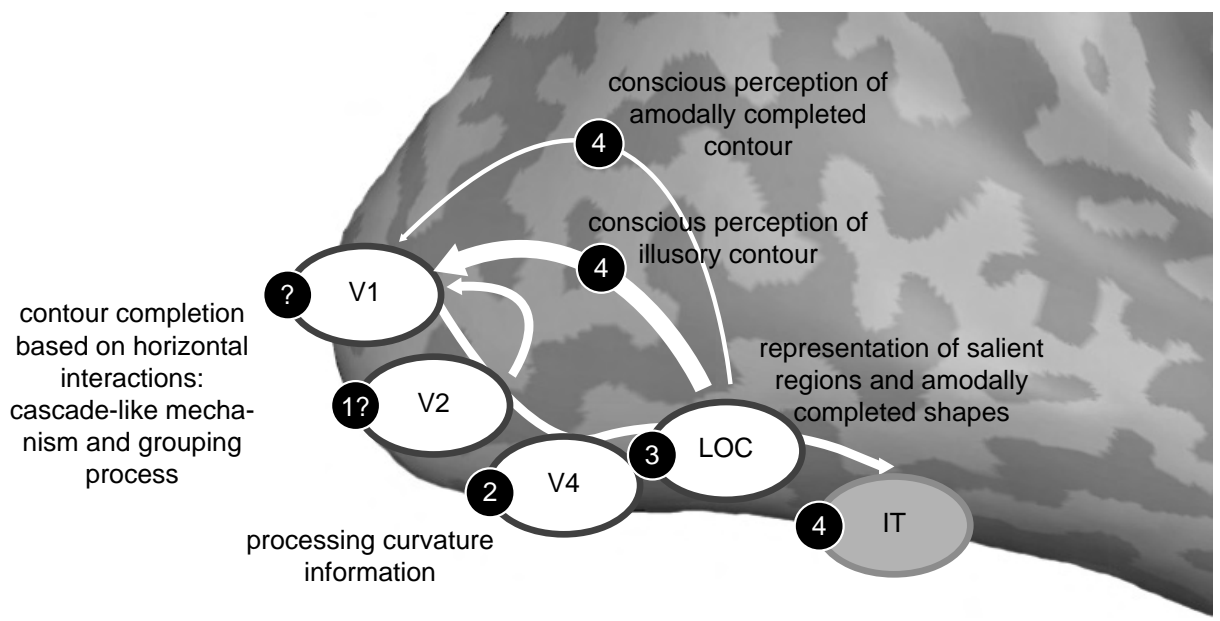

Figure 6. A putative combined model for amodal and modal completion. Amodal and modal completion both relay on contour completion mechanisms in early visual cortex, most presumably in V2. Along the ventral pathway, V4 then processes curvature information. Higher-level visual area LOC represents salient regions and amodally completed shapes. Feedback connections from $\mathrm{LOC}$ to area $\mathrm{V} 1$ are the basis for the conscious percept of an amodally completed or illusory contour. Feedback connections from V2 to V1 might enhance contour-related processing. The tentative importance of connections are coded by the thickness of the arrows.

in the case of perceptual learning (Li et al., 2008). A combined feedback signal might strengthen the responses in V1 even more. Furthermore, the pattern of activity in V1 and V2 might disambiguate the type of contour one is currently looking at. Ramsden et al. (2001) found the pattern of activity to be correlated between V1 and V2 in the case of a real contour, while it was out-of-phase for an illusory contour (Ramsden et al., 2001). Thus, activity in V1 might rely on different feedback signals from V2 and higher-visual areas that serve different purposes. Finally, in both cases, amodal and modal completion, I assume that the signal is further passed to IT cortex and from there to frontal cortex. 


\section{Patterns of $f M R I$ activity representing perceived direction in ap- parent motion}

In our study on apparent motion described in Chapter 5 we identified several brain regions being involved in the processing of long-range apparent motion. Most importantly, using MVPA we were able to decode the perceived direction of motion from activity in inferior frontal gyrus (IFG), right temporal parietal junction (TPJ), right premotor cortex (PMC) as well as areas surrounding hMT+ and early visual areas. Thus, those regions seem to play a crucial role in representing the perceived direction in long-range apparent motion. In the following I will discuss potential functional roles of the various brain regions that are involved in apparent motion. In the end, I aim at deriving a putative neuronal model for long-range apparent motion.

\section{Brain regions involved in apparent motion}

Various brain regions have been identified as being involved in processing apparent motion. Those regions are early visual areas (V1, V2, V3), motion-sensitive areas hMT+, $\mathrm{V} 3 \mathrm{a}$, and $\mathrm{KO}$ of the dorsal processing pathway, color-sensitive region hV4, and shapesensitive area LOC of the ventral processing pathway. Furthermore, regions in the most anterior portion of the temporal pole as well as parietal and frontal areas have been found to contribute to apparent motion processing.

hMT+

As I have already elaborated in the introductory chapter, hMT+ plays an important role in processing apparent motion. It has been identified as the key player in apparent motion in electrophysiological (Mikami, 1991; Newsome et al., 1986), neuroimaging (Goebel et al., 1998; Larsen et al., 2006; Liu et al., 2004; Muckli et al., 2002, 2005; Sterzer and Kleinschmidt, 2007; Sterzer et al., 2003; Tse, 2006) as well as interference studies (Matsuyoshi et al., 2007). Primarily, hMT+ shows switch-related activity, i.e. an increase in activity when a perceptual switch occurs from a stationary (flickering) to a moving pattern (Muckli et al., 2002; Sterzer et al., 2003). This switch-related response seems to be independent of the type of stimulus and hence the type of apparent motion, since it does occur with motion quartet stimuli evoking translational apparent motion (Sterzer et al., 2003) as well as with the spinning wheel illusion that elicits radial apparent motion (Sterzer et al., 2002). Furthermore, Sterzer and Kleinschmidt (2007) found that hMT+ shows switch-related activity independent of whether the switch occurs spontaneously (as in the ambiguous motion quartet) or whether it is induced by the stimulus (as in an unambiguous motion quartet). Matsuyoshi et al. (2007) applied rTMS over hMT+ or a control region over inferotemporal cortex while subjects viewed apparent motion. The stable percept of apparent motion was markedly reduced during the concomitant application of rTMS over hMT+ but not over the control region. Large receptive field sizes enable hMT+ to integrate motion signals across large portions of the visual field (Mikami et al., 1986b). A further plus is the fast input from subcortical structures (bypassing $\mathrm{V} 1$ ) that support ultra quick motion detection (ffytche et al., 1995). Thus, it has been argued that hMT+ pools responses of $\mathrm{V} 1$ neurons based on spatiotemporal summation (Grossberg and Rudd, 1992), thereby solving the correspondence problem (Larsen et al., 2006) in long-range apparent motion. 


\section{Early visual areas}

First evidence for switch-related activity in early visual cortex was only found in singlesubject analyses (Muckli et al., 2002). Later, Muckli et al. (2005) demonstrated that activity related to apparent motion can be found already in primary visual cortex. More precisely, they localized activity corresponding to the apparent motion trace in retinotopically defined regions in V1. Moreover, Muckli et al. (2005) showed that this activity is independent from the attentional focus, i.e. it occurs also when attention is drawn to a demanding center task. Larsen et al. (2006) as well as Sterzer et al. (2006) replicated these findings. In a former study, Liu and coworkers (2004) failed to show activity in V1 that is related to the motion trace. These contradicting results might be due to different visual stimulations (Muckli et al., 2006). The three studies that were able to identify activity in V1 that corresponds to the apparent-motion trace used translational apparent motion, while Liu et al. (2004) employed radial apparent motion. The trace-sensitive activity in V1 has been related to perceptual filling-in of the illusory motion path (Muckli et al., 2005; Sterzer et al., 2006). It has been suggested (Muckli et al., 2005; Yantis and Nakama, 1998) and then shown (Sterzer et al., 2006) that the activity in primary visual cortex reflects feedback signals from higher visual areas, more precisely hMT+. Sterzer et al. (2006) used dynamic causal modeling to demonstrate that trace-related activity in V1 is based on enhanced feedback from hMT+. They tested different possible models that might account for the trace-related activity, and found that a model containing strong direct feedback from hMT+ to V1 is more likely than lateral connections within V1 (Sterzer et al., 2006).

\section{Parietal regions}

The involvement of parietal regions in apparent motion processing has also been shown repeatedly (Goebel et al., 1998; Sterzer et al., 2002; 2003; Sterzer and Kleinschmidt, 2007; Williams et al., 2003). Goebel et al. (1998) identified regions of the inferior and superior parietal lobule to be involved in apparent motion. However, the same regions were similarly active during flicker perception. Sterzer and colleagues (2002; 2003) demonstrated that switch-related activity in area KO depended on the type of stimuli in use. KO shows switch-related activity for radial apparent motion (2002), but not (or less so) for translational apparent motion (2003). Thus, our results reported in Chapter 5 extend the current knowledge on the role of parietal cortex in apparent motion perception extensively, by demonstrating that activity from parts of IPS and TPJ contains information on the perceived illusory motion direction. It is known that IPS/TJP receive input from $\mathrm{hMT}+$ and early visual areas directly (Colby et al., 1993). Especially, human V3A and IPS-0/V7 have been identified as motion-sensitive regions (Orban et al., 2003). Although the functional role of those parietal regions in apparent motion has yet to be established, it is conceivable that they contribute to spatial attention mechanisms.

\section{Frontal regions}

The role of frontal regions in apparent motion processing is controversial. In fact, frontal regions have exhibited switch-related activity (Muckli et al., 2002; Sterzer and Kleinschmidt, 2007). Muckli et al. (2002) found switch-related activity in sensorimotor and somatosensory cortex, insular cortex, supplemental motor area (SMA), and middle 
frontal cortex. They attributed most of the activity to the preparation and execution of motor responses. Sterzer and Kleinschmidt (2007) found switch-related activity in bilateral inferior frontal cortex, SMA and left sensorimotor cortex. Moreover, they contrasted an ambiguous motion quartet stimulus with an unambiguous motion stimulus. Sterzer and Kleinschmidt (2007) demonstrated that a region in bilateral IFG shows higher activity for spontaneous perceptual switches that occur with the ambiguous motion quartet than for perceptual switches that are induced by an unambiguous motion stimulus. The same region in the right hemisphere also shows the earliest response to the spontaneous perceptual switch in comparison to all other regions involved (including hMT+). In direct contrast to Muckli et al., (2002; 2005) Sterzer and Kleinschmidt (2007) interpret their data in favor of a neuronal model of apparent motion where perceptual switches are initiated in inferior frontal cortex and fed back to $\mathrm{hMT}+$ and other areas. I will discuss this issue in our putative neuronal model of longrange apparent motion.

\section{Areas in ventral visual cortex}

At least three regions in ventral visual cortex have been found to be involved in apparent motion, mainly in cases where transformational apparent motion has been applied. Transformational apparent motion can be defined as apparent motion between two objects differing in shape or color. Observers perceive apparent motion and the same time a smooth transformation from one object into the other. Color-sensitive region hV4 is activated if the apparent motion stimulus contains a color transformation (Sterzer and Kleinschmidt, 2005). The LOC contributes to apparent motion processing in the case of shape transformation (Liu et al., 2004; Tse, 2006). Interestingly, Liu et al. (2004) showed that parts of the LOC that are sensitive to apparent motion seem to overlap with hMT+. Zhuo et al. (2003) have identified a region in the anterior-most portion of the temporal lobe (aTG) that is specific for transformational long-range apparent motion. aTG does not respond to short-range apparent motion. In the case of transformational long-range apparent motion, the signal varies with the structural difference between the two objects bound in motion. The more different they are (a square and a ring in comparison to a square and a rhomboid) the higher the signal in aTG (Zhuo et al.,2003). Thus, whenever apparent motion is accompanied by a change in either color or shape, ventral visual areas are involved. It has been suggested that they support object continuity by maintaining object representation through spatiotemporal transformations (Liu et al., 2004). The involvement of ventral areas in transformational apparent motion can be conceived as a hint that this type of apparent motion is in the middle of a possible continuum between pure apparent motion and apparent rotation. Both, transformational apparent motion as well as apparent rotation require shape transformations. However, transformational apparent motion is set in 2D, while apparent rotation is set in 3D. The third dimension might therefore require an even more elaborate interaction between dorsal and ventral visual areas to support object representation through spatiotemporal transformations.

\section{A putative model for long-range apparent motion}

Figure 7 shows a summary of the aforementioned neurophysiological findings and a depiction of a putative model for long-range apparent motion. Besides the labels 
indicating the potential role of the specific region in the model, I have included pictograms that indicate how the regions were identified to contribute to apparent motion (see figure legend). Almost all areas show activity related to perceptual switches. Only a subset of these areas, however contain information on the perceived direction of apparent motion as has been reported in Chapter 5. Those areas are therefore even more correlated with the perceptual content of apparent motion. Areas that show switchrelated activity but do not represent the perceived direction are most likely involved in the preparation and execution of motor responses. Ventral visual areas are engaged in apparent motion as soon as it requires a color or shape transformation for the apparent motion to be perceived. Early visual cortex demonstrates trace-related activity in a retinotopic fashion. It is now agreed upon that this activity reflects a perceptual

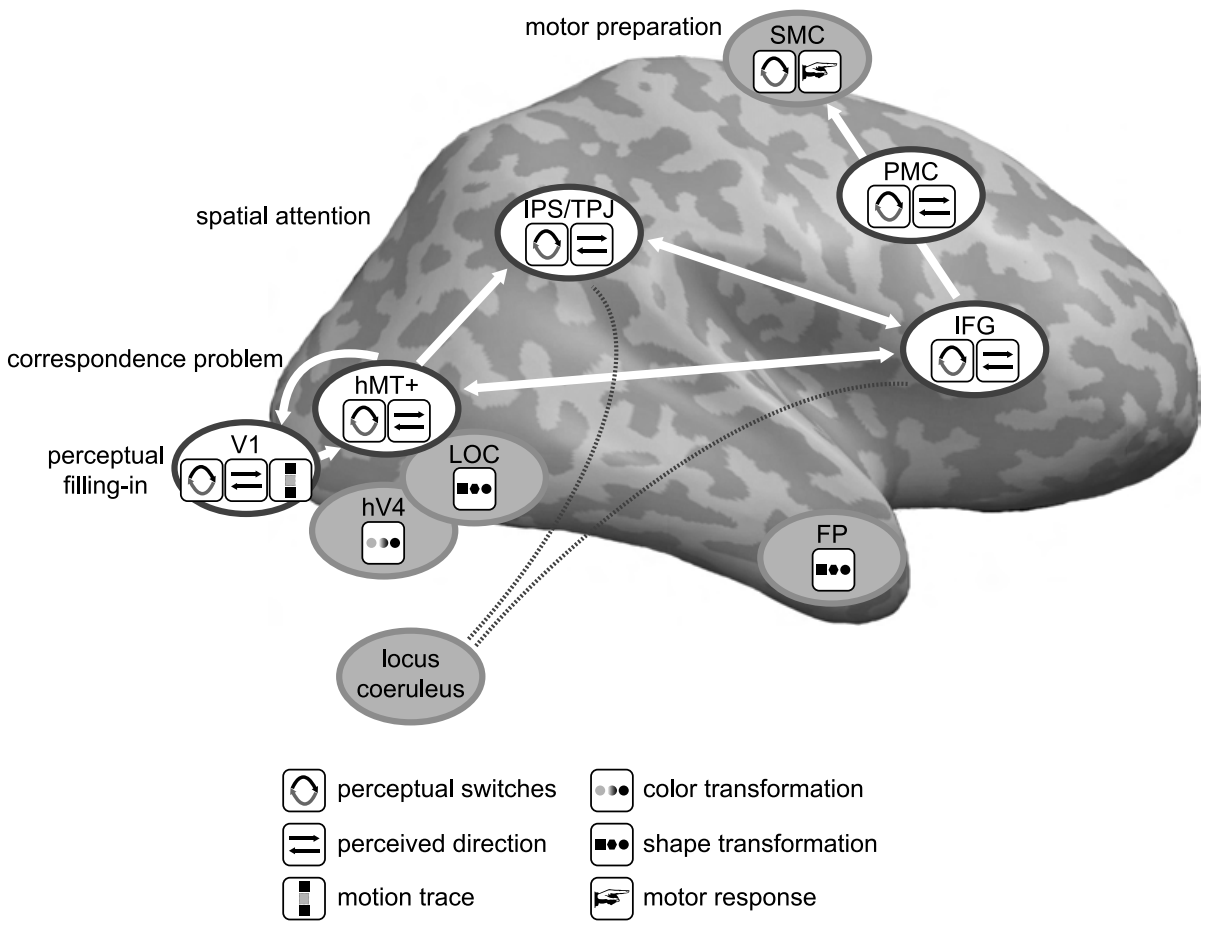

Figure 7. Summary of neuroimaging findings on apparent motion and a putative neuronal model. Apparent motion is critically processed by neurons in hMT+ that pool responses of $\mathrm{V} 1$ input neurons, thereby solving the correspondence problem. Activity from hMT+ is fed back to primary visual cortex generating a perceptual filling-in of the illusory motion trace. Ventral visual cortex is active when color or shape transformations are required for the perception of apparnt motion. The role of the frontoparietal network is still under debate. We hypothesize that noradrenergic input form the locus coeruleus might signal a non-specific switch of perceptual states, while the specific perceptual content of the switch is set in regions that represent the critical visual feature, e.g. motion direction (see text for discussion). Premotor regions are involved in the preparation and execution of motor responses. 
filling-in that is fed back from hMT+ (Larsen et al., 2006; Muckli et al., 2005; Sterzer et al., 2006).

A critical question for a putative model for long-range apparent motion is where and how perceptual switches are initiated. Two opposing views have been prominent in the recent literature and a third view might emerge from our data reported in Chapter 5: The first view claims that perceptual switches occur due to competition or neuronal adaptation mechanisms (Anstis et al., 1985; Clatworthy and Frisby, 1973; Finlay and von Grünau, 1987; Muckli et al., 2002). For the case of the bistable apparent motion quartet this would imply that neuronal populations coding for one motion direction adapt over time, and once a critical threshold is reached, opposing neuronal populations coding for the other motion direction take over (and vice versa). These adaptation mechanisms are likely to occur at the level of hMT+ (Anstis et al., 1985; Muckli et al., 2002), since this area contains the highest amount of direction-selective cells (Lennie, 1998). This view states that perceptual switches are based on bottom-up, hard-wired processes.

The contrasting second view claims that perceptual switches are initiated in frontal cortices (Sterzer and Kleinschmidt, 2007). More precisely, Sterzer and Kleinschmidt (2007) argue that higher-order processes may manipulate local processes such as the aforementioned adaptation processes, thereby initiating the perceptual switches. It remains unclear, however, how these higher-order processes sense and target the local processes, and why local processing per se is not sufficient for the initiation of a perceptual switch. Our data reported in Chapter 5 shows that percept-related content is represented in IFG. This suggests that this region has not only the role to initiate perceptual switches non-specifically, but that it contains information about the specific perceptual contents of conscious experience.

As alluded to in Chapter 5 parietal and frontal regions might also be involved in a more general attentional network (Corbetta et al., 2008). Based on the known connections of this attentional network to structures of the brain stem - namely, the locus coeruleus - we hypothesize that this noradrenergic system might initiate a non-specific perceptual switch. Connected areas in frontal and parietal cortices might then translate this non-specific switch signal into a switch between specific perceptual contents, e.g. perceived motion directions. Between which perceptual contents the switch occurs exactly might depend on interactions between specific brain regions representing these visual features (direction-selective brain regions).

Kohler and colleagues (2008) recently demonstrated that on the one hand phase durations of perceptual switches can be voluntarily influenced and are highly susceptible to attentional modulations. On the other hand, the percept is never fully under the observer's will - spontaneous switches still occur (Kohler et al., 2008). In conclusion, the dynamics of perceptual switches seem to be partly based on top-down influences and partly based on bottom-up, hard-wired processes.

\section{Conscious visual perception}

Ultimately all studies presented in this dissertation deal with conscious visual perception. What I have shown so far are different networks of brain regions being involved in 
conscious visual perception. I have also demonstrated that the same brain regions that code veridical visual perception are active during illusory perception. That is, I have shown that these regions code the perceptual content also in cases where physical stimulation is lacking. And still, THE intriguing question remains unanswered: where, when and how does consciousness come into play?

Obviously, the answer to this question requires further investigations and most probably a multi-method approach where neuroimaging techniques are complemented by methods such as TMS or microstimulation that provide the possibility of directly interfering with brain processes. Thereby - and also by neuropsychological case studies - one might unravel e.g. which brain areas are necessary and sufficient for conscious visual perception. Frontoparietal networks have been associated with the emergence of visual awareness (Baars, 2002; Dehaene et al., 2001) as has been primary visual cortex (Rees et al., 2002; Tong, 2003; Tong and Engel, 2001). Recently, feedback activity from higher to lower visual areas has been identified as a putative mechanism coding visual consciousness (Fahrenfort et al., 2008; Lamme, 2006; Silvanto et al., 2005; Supèr et al., 2001). I have the tendency to favor the last approach. More precisely, I hypothesize that the high decoding performance for the perceived direction in longrange apparent motion in early visual areas might reflect feedback signal from hMT+ gating conscious perception. It would be very interesting to investigate whether we are also able to decode the perceived direction in apparent rotation from activity in early visual areas. Furthermore, I have already suggested that in the case of modal and amodal completion, feedback from ventral visual areas to primary visual cortex might elicit the conscious perception of illusory and completed contours.

In the end a fruitful combination of smart experimental designs, cutting-edge research techniques as well as a little bit of luck will get us closer to a better understanding of conscious visual perception. 

Summary 
The central theme of the present dissertation is human visual perception. More precisely, we investigated cortical processes underlying the conscious perception of visual illusions. Brain activity was assessed by employing functional magnetic resonance imaging (fMRI), a non-invasive neuroimaging technique that detects changes in blood flow related to neuronal activity. We met the challenge of identifying brain activity related to illusory perception by utilizing recent advancements in neuroimaging: $f M R I$ adaptation and multi-voxel pattern analysis (MVPA).

FMRI adaptation describes the fact that the fMRI signal attenuates due to repeated stimulation in the particular brain region representing the stimulus. The trick using fMRI adaptation in the study of visual illusions works as follows: First, the stimulus eliciting the visual illusion is presented. Second, a test stimulus is presented. This test stimulus can either reflect the illusory percept or depict the actual physical stimulus. The rationale is that brain regions representing the illusory percept should show an adapted signal to the presentation of the 'illusory' test stimulus, while brain regions representing the physical stimulus should show an adapted signal to the presentation of the 'physical' stimulus.

MVPA denotes mathematical algorithms which extract patterns of activity from fMRI data. The functional architecture of the brain consists of cortical representations that are distributed and highly overlapping. Multivariate statistics implemented in MVPA have to be used to extract these patterns of activity, since classical fMRI data analysis using univariate statistics fails. MVPA is often referred to as a 'brain reading' or 'mind reading' device, since it can be employed to decode perceptual or cognitive states out of brain data - also illusory ones.

Chapter 1 provides an introductory overview of $\mathrm{FMRI}$ adaptation and MVPA as well as of the three visual phenomena under study, namely apparent motion, apparent rotation and amodal completion.

The method of fMRI adaptation is further evaluated in Chapter $\mathbf{2}$ where we present a detailed review of papers in the domain of visual neuroscience that made use of $\mathrm{fMRI}$-adaptation paradigms. More precisely, we focus on methodological considerations concerning experimental design, stimulus presentation and influencing factors such as awareness and attention. We reveal that different fMRI-adaptation designs capture heterogeneous neuronal adaptation effects and that one has to keep this in mind when interpreting null-effects in fMRI-adaptation studies.

In Chapter 3 we report on our neuroimaging studies on the phenomenon of apparent rotation. Apparent rotation refers to a visual illusion of rotation of three-dimensional objects. Although only two perspectives of the object are presented in rapid succession, observers perceive the illusion of a smoothly moving object. The missing, intermediate rotation positions are filled in by the visual system. Using fMRI adaptation we show that a cortical network comprising regions of the ventral and dorsal visual processing streams represents those intermediate object views. More precisely, we demonstrate $\mathrm{fMRI}$-adaptation in these regions to the illusory intermediate rotation positions (but not to outside positions), as if these positions had been actually presented. In control experiments we confirm that these findings are really based on the motion illusion, and we evaluate the influence of the behavioral task and attentional mechanisms. We provide an extensive discussion of the brain regions involved in the 
cortical network as well as a tentative model of apparent rotation in the discussion at the end of the thesis.

Our fMRI experiments on amodal completion are presented in Chapter 4. Amodal completion refers to one of the most fundamental mechanisms in human vision: the automatic perceptual completion of partly occluded objects. Using fMRI-adaptation we reveal separate cortical stages in amodal completion:While early visual cortex processes the local contour information of the physical stimulus, regions in inferior temporal cortex represent the completed shape. Again we demonstrate fMRI-adaptation effects to the (illusory) completed object as if this object had been actually presented. In addition, our findings suggest that at the level of inferior temporal cortex the original contour information is not preserved but replaced by the completed percept. We compare our results on amodal completion with other studies on the related phenomenon of modal completion or illusory contours and propose a combined model for the two phenomena.

Chapter 5 contains our $\mathrm{FMRI}$ investigation on apparent motion utilizing MVPA. Apparent motion refers to the illusion of one stimulus moving between two locations, although only two stimuli are presented alternately at these locations. We made use of the 'motion quartet' - an ambiguous stimulus that induces either horizontal or vertical apparent motion. MVPA was used to decode the perceived direction from activity patterns in occipital, parietal and frontal regions. These regions have been identified before to show activity related to perceptual switches in apparent motion. We found high decoding performance for regions at the temporal parietal junction, inferior frontal gyrus and near hMT+. We were also able to extract the percptual state from activity in early visual areas. Based on these findings we discuss a putative model for long-range apparent motion.

Chapter 6 serves the purpose of extending the discussions of the antecedent chapters with the final aim of proposing cortical models for each of the visual phenomena under study. The thesis closes with a brief comment on a potential neuronal correlate of conscious visual perception. 



\section{Samenvatting}


Het centrale thema van deze dissertatie is de menselijke visuele waarneming. Om precies te zijn onderzochten we de processen in de hersenschors (cortex) die ten grondslag liggen aan de waarneming van visuele illusies. Hersenactiviteit werd in beeld gebracht door middel van functionele magnetische resonantie (fMRI), een niet-ingrijpende beeldvormende techniek die aan neurale activiteit gerelateerde veranderingen in de doorbloeding meet. De uitdaging om hersenactiviteit te identificeren die samenhangt met visuele illusies gingen we aan door gebruik te maken van recente ontwikkelingen op het gebied van functionele beeldvorming van de hersenen: $\mathrm{FMRI}-$ adaptatie en multi-voxel patroonanalyse (MVPA).

FMRI-adaptatie berust op het gegeven dat het fMRI-signaal daalt bij herhaalde stimulatie van het specifieke hersengebied dat een stimulus representeert. De truc van het gebruik van fMRI-adaptatie ter bestudering van visuele illusies werkt als volgt: eerst wordt de stimulus die de visuele illusie opwekt aangeboden. Daarna wordt een teststimulus aangeboden. De teststimulus kan ofwel overeenkomen met de schijnwaarneming, of de echte fysieke stimulus weergeven. De achterliggende redenering is dat hersengebieden die de schijnwaarneming representeren een geadapteerd signaal zouden moeten laten zien bij aanbieding van de 'illusoire' teststimulus, terwijl hersengebieden die de fysieke stimulus representeren een geadapteerd signaal zouden moeten laten zien bij aanbieding van de 'fysieke' stimulus.

MVPA is de verzamelnaam voor mathematische algoritmes die activatiepatronen uit fMRI-data kunnen destilleren. De functionele architectuur van de hersenen wordt gekenmerkt door gedistribueerde en overlappende corticale representaties. Multivariate statistiek, geïmplementeerd in MVPA, moet gebruikt worden om deze activatiepatronen uit elkaar te halen, omdat klassieke fMRI data-analyse die univariate statistiek gebruikt hier tekort schiet. Men verwijst vaak naar MVPA als een methode om de hersenen of de geest te 'lezen', omdat het ingezet kan worden om perceptuele of cognitieve toestanden uit hersendata te decoderen - ook illusoire.

Hoofdstuk 1 geeft een inleidend overzicht van zowel fMRI-adaptatie en MVPA als de drie bestudeerde visuele verschijnselen, te weten schijnbeweging, schijnrotatie en amodale completering.

Op fMRl-adaptatie wordt verder ingegaan in hoofdstuk 2 waarin we een gedetailleerde bespreking presenteren van studies op het gebied van de visuele neurowetenschappen die gebruik maken van fMRI-adaptatie. We richten ons, om precies te zijn, op methodologische overwegingen wat betreft de experimentele opzet, stimulusaanbieding, en beïnvloedende factoren zoals bewustzijn en aandacht. We maken duidelijk dat verschillende fMRl-adaptatie onderzoeksopzetten uiteenlopende neuronale adaptatie-effecten vangen en dat men hiermee rekening dient te houden bij de interpretatie van uitblijvende effecten in fMRI-adaptatie studies.

In hoofdstuk 3 berichten we over onze beeldvormende studie van het fenomeen schijnrotatie. Schijnrotatie verwijst naar een visuele rotatie-illusie van driedimensionale voorwerpen. Hoewel het beeld van het voorwerp slechts vanuit twee perspectieven in snelle opeenvolging aangeboden wordt, heeft de toeschouwer de illusie dat het voorwerp een vloeiende beweging maakt. De ontbrekende, tussenliggende posities worden ingevuld door het visuele systeem. Met behulp van fMRI-adaptatie laten we zien dat deze tussenliggende posities gerepresenteerd worden door een corticaal netwerk bestaande uit gebieden van de ventrale en dorsale visuele verwerkingsroute. 
Om precies te zijn tonen we aan dat er in deze gebieden fMRI-adaptatie is voor de tussenliggende rotatieposities (maar niet voor posities daarbuiten), als waren deze posities werkelijk aangeboden. In controle-experimenten verzekeren we ons ervan dat deze bevindingen echt gebaseerd zijn op de bewegingsillusie, en beoordelen we de invloed van de gedragstaak en aandachtsmechanismen. We gaan uitgebreid in op de hersengebieden in het corticale netwerk en doen in de discussie aan het eind van de dissertatie ook een eerste poging tot het formuleren van een model van schijnrotatie.

Onze fMRI-experimenten over amodale completering worden gepresenteerd in hoofdstuk 4. Amodale completering verwijst naar één van de meest fundamentele mechanismen in de menselijke visuele waarneming: de automatische perceptuele completering van gedeeltelijk bedekte voorwerpen. Met fMRI-adaptatie leggen we afzonderlijke corticale stadia in amodale completering bloot: terwijl de vroege visuele cortex de plaatselijke contour- informatie van de fysieke stimulus verwerkt, representeren delen van de inferieure temporele hersenschors de volledige vorm. Opnieuw laten we fMRI adaptatie-effecten zien voor het illusoire volledige voorwerp, als ware dit voorwerp werkelijk aangeboden. Bovendien suggereren onze bevindingen dat op het niveau van de inferieure temporele hersenschors de oorspronkelijke contour-informatie niet behouden blijft, maar vervangen wordt door de vervolledigde waarneming. We vergelijken onze resultaten met betrekking tot amodale completering met andere studies over het verwante verschijnsel modale completering of schijncontouren en opperen een gecombineerd model voor de twee fenomenen.

Hoofdstuk 5 bevat ons fMRI-onderzoek naar schijnbeweging dat gebruik maakt van MVPA. Schijnbeweging verwijst naar de illusie van één stimulus die tussen twee locaties beweegt, hoewel in werkelijkheid op deze locaties afwisselend twee stimuli gepresenteerd worden. We maakten gebruik van het 'bewegingskwartet' - een ambigue stimulus die ofwel een horizontale of een verticale schijnbeweging opwekt. MVPA werd gebruikt om de waargenomen richting te ontcijferen uit activatiepatronen in occipitale, parietale en frontale gebieden. Eerder is activiteit in deze gebieden in verband gebracht met richtingsveranderingen van de waargenomen schijnbeweging. Wij vonden goede ontcijferingsprestaties voor gebieden op de temporele-parietale kruising, de inferieure frontale hersenplooi en dichtbij hMT+. We waren ook in staat om informatie over de waarnemingstoestand te onttrekken uit vroege visuele gebieden. Op basis van deze bevindingen bespreken we een mogelijk model voor schijnbeweging over langere afstanden.

Hoofdstuk 6 dient om de discussies van de voorafgaande hoofdstukken uit te breiden met als uiteindelijke doel corticale modellen te presenteren voor elk van de onderzochte visuele verschijnselen. De dissertatie sluit af met een korte kanttekening over een mogelijke neuronale correlaat van bewuste visuele waarneming. 



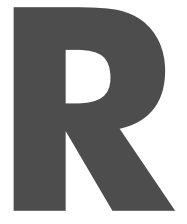

\section{References}


Allman JM, Kaas JH (1971) A representation of the visual field in the caudal third of the middle temporal gyrus of the owl monkey (Aotus trivirgatus). Brain Res 31:85-105.

Anderson BL, Singh M, Fleming RW (2002) The interpolation of object and surface structure. Cognit Psychol 44:148-190.

Anderson BL (2007a) The demise of the identity hypothesis and the insufficiency and nonnecessity of contour relatability in predicting object interpolation: comment on Kellman, Garrigan, and Shipley (2005). Psychol Rev 114:470-487.

Anderson BL (2007b) Filling-in models of completion: rejoinder to Kellman, Garrigan, Shipley, and Keane (2007) and Albert (2007). Psychol Rev 114:509-527.

Andrews TJ, Ewbank MP (2004) Distinct representations for facial identity and changeable aspects of faces in the human temporal lobe. Neuroimage 23:905-913.

Anstis SM, Giaschi D, Cogan Al (1985) Adaptation to apparent motion. Vision Res 25:1051-1062.

Ashida H, Lingnau A, Wall MB, Smith AT (2007) fMRI adaptation reveals separate mechanisms for first-order and second-order motion. J Neurophysiol 97:1319-1325.

Aston-Jones G, Cohen JD (2005) An integrative theory of locus coeruleus-norepinephrine function: adaptive gain and optimal performance. Annu Rev Neurosci 28:403-450.

Baars BJ (2002) The conscious access hypothesis: origins and recent evidence. Trends Cogn Sci 6:47-52.

Baker CL, Hess RF, Zihl J (1991) Residual motion perception in a 'motion-blind' patient, assessed with limited-lifetime random dot stimuli.J Neurosci 11:454-461.

Bakin JS, Nakayama K, Gilbert CD (2000) Visual responses in monkey areas V1 and V2 to three-dimensional surface configurations. J Neurosci 20:8188-8198.

Bandettini PA, Kwong KK, Davis TL, Tootell RB, Wong EC, Fox PT, Belliveau JW, Weisskoff RM, Rosen BR (1997) Characterization of cerebral blood oxygenation and flow changes during prolonged brain activation. Hum Brain Mapp 5:93-109.

Beckers G, Hömberg V (1991) Impairment of visual perception and visual short term memory scanning by transcranial magnetic stimulation of occipital cortex. Exp Brain Res 87:421-432.

Beckers G, Hömberg V (1992) Cerebral visual motion blindness: transitory akinetopsia induced by transcranial magnetic stimulation of human area V5. Proc R Soc Lond B Biol Sci 249:173-178.

Bentley P,Vuilleumier P, Thiel CM, Driver J, Dolan RJ (2003) Effects of attention and emotion on repetition priming and their modulation by cholinergic enhancement. $J$ Neurophysiol 90:1171-1181.

Biederman I, Gerhardstein PC (1993) Recognizing depth-rotated objects: evidence and conditions for three-dimensional viewpoint invariance. J Exp Psychol Hum Percept Perform 19:1162-1182.

Biederman I, Bar M (1999) One-shot viewpoint invariance in matching novel objects. Vision Research 39:2885-2899.

Blake R, Tadin D, Sobel KV, Raissian TA, Chong SC (2006) Strength of early visual adaptation depends on visual awareness. Proc Natl Acad Sci U S A 103:4783-4788.

Boynton GM, Engel SA, Glover GH, Heeger DJ (1996) Linear systems analysis of functional magnetic resonance imaging in human V1. J Neurosci 16:4207-4221. 
Boynton GM, Demb JB, Glover GH, Heeger DJ (1999) Neuronal basis of contrast discrimination. Vision Res 39:257-269.

Boynton GM, Finney EM (2003) Orientation-specific adaptation in human visual cortex. J Neurosci 23:8781-8787.

Bremmer F, Schlack A, Shah NJ, Zafiris O, Kubischik M, Hoffmann K, Zilles K, Fink GR (2001) Polymodal motion processing in posterior parietal and premotor cortex: a human fMRI study strongly implies equivalencies between humans and monkeys. Neuron 29:287-296.

Brewer AA, Liu J, Wade AR, Wandell BA (2005) Visual field maps and stimulus selectivity in human ventral occipital cortex. Nat Neurosci 8:1102-1109.

Brouwer GJ, van Ee R (2007) Visual cortex allows prediction of perceptual states during ambiguous structure-from-motion.J Neurosci 27:1015-1023.

Buckner RL, Petersen SE, Ojemann JG, Miezin FM, Squire LR, Raichle ME (1995) Functional anatomical studies of explicit and implicit memory retrieval tasks. J Neurosci 15:12-29.

Buckner RL, Goodman J, Burock M, Rotte M, Koutstaal W, Schacter D, Rosen B, Dale AM (1998) Functional-anatomic correlates of object priming in humans revealed by rapid presentation event-related fMRI. Neuron 20:285-296.

Bullmore E, Brammer M, Williams SC, Rabe-Hesketh S, Janot N, David A, Mellers J, Howard R, Sham P (1996) Statistical methods of estimation and inference for functional MR image analysis. Magn Reson Med 35:261-277.

Bülthoff HH, Edelman S (1992) Psychophysical support for a two-dimensional view interpolation theory of object recognition. Proc Natl Acad Sci U S A 89:60-64.

Bunzeck N, Schütze H, Düzel E (2006) Category-specific organization of prefrontal response-facilitation during priming. Neuropsychologia 44:1765-1776.

Chen CM, Lakatos P, Shah AS, Mehta AD, Givre SJ, Javitt DC, Schroeder CE (2007) Functional anatomy and interaction of fast and slow visual pathways in macaque monkeys. Cereb Cortex 17:1561-1569.

Claeys KG, Lindsey DT, De Schutter E, Orban GA (2003) A higher order motion region in human inferior parietal lobule: evidence from fMRI. Neuron 40:621-642.

Clatworthy JL, Frisby JP (1973) Real and apparent visual movement: Evidence for a unitary mechanism. Perception 2:161-164.

Clifford CW, Webster MA, Stanley GB, Stocker AA, Kohn A, Sharpee TO, Schwartz O (2007) Visual adaptation: Neural, psychological and computational aspects. Vision Res 47:3125-3131.

Cohen MS (1997) Parametric analysis of fMRI data using linear systems methods. Neuroimage 6:93-103.

Colby CL, Duhamel JR, Goldberg ME (1993) Ventral intraparietal area of the macaque: anatomic location and visual response properties. J Neurophysiol 69:902-914.

Corbetta M, Kincade JM, Ollinger JM, McAvoy MP, Shulman GL (2000) Voluntary orienting is dissociated from target detection in human posterior parietal cortex. Nat Neurosci 3:292-297.

Corbetta M, Patel G, Shulman GL (2008) The reorienting system of the human brain: from environment to theory of mind. Neuron 58:306-324.

Corbetta M, Shulman GL (2002) Control of goal-directed and stimulus-driven attention in the brain. Nat Rev Neurosci 3:201-215. 
Courtney SM, Petit L, Maisog JM, Ungerleider LG, Haxby JV (1998) An area specialized for spatial working memory in human frontal cortex. Science 279:1347-1351.

Cox DD, Savoy RL (2003) Functional magnetic resonance imaging (fMRI) "brain reading": detecting and classifying distributed patterns of fMRI activity in human visual cortex. Neuroimage 19:261-270.

Culham JC, Brandt SA, Cavanagh P, Kanwisher NG, Dale AM, Tootell RB (1998) Cortical fMRI activation produced by attentive tracking of moving targets. J Neurophysiol 80:2657-2670.

Dale AM, Buckner RL (1997) Selective averaging of rapidly presented individual trials using fMRI. Human Brain Mapping 5:329-340.

De Weerd P, Sprague JM, Raiguel S, Vandenbussche E, Orban GA (1993) Effects of visual cortex lesions on orientation discrimination of illusory contours in the cat. Eur J Neurosci 5:1695-1710.

De Weerd P, Desimone R, Ungerleider LG (1996) Cue-dependent deficits in grating orientation discrimination after V4 lesions in macaques. Vis Neurosci 13:529-538.

De Wit TC, Bauer M, Oostenveld R, Fries P, van Lier R (2006) Cortical responses to contextual influences in amodal completion. Neuroimage 32:1815-1825.

Dehaene S, Naccache L, Cohen L, Bihan DL, Mangin JF, Poline JB, Rivière D (2001) Cerebral mechanisms of word masking and unconscious repetition priming. Nat Neurosci 4:752-758.

Deruelle C, Barbet I, Depy D, Fagot J (2000) Perception of partly occluded figures by baboons (Papio papio). Perception 29:1483-1497.

Dobbins IG, Schnyer DM, Verfaellie M, Schacter DL (2004) Cortical activity reductions during repetition priming can result from rapid response learning. Nature 428:316319.

Dragoi V, Sharma J, Sur M (2000) Adaptation-induced plasticity of orientation tuning in adult visual cortex. Neuron 28:287-298.

Dubner R, Zeki SM (1971) Response properties and receptive fields of cells in an anatomically defined region of the superior temporal sulcus in the monkey. Brain Res 35:528-532.

Dupont P, De Bruyn B, Vandenberghe R, Rosier AM, Michiels J, Marchal G, Mortelmans L, Orban GA (1997) The kinetic occipital region in human visual cortex. Cereb Cortex 7:283-292.

Eddy MD, Schnyer D, Schmid A, Holcomb PJ (2007) Spatial dynamics of masked picture repetition effects. Neuroimage 34:1723-1732.

Eger E, Schyns PG, Kleinschmidt A (2004a) Scale invariant adaptation in fusiform faceresponsive regions. Neuroimage 22:232-242.

Eger E, Henson RN, Driver J, Dolan RJ (2004b) BOLD repetition decreases in object-responsive ventral visual areas depend on spatial attention. J Neurophysiol 92:12411247.

Eger E, Schweinberger SR, Dolan RJ, Henson RN (2005) Familiarity enhances invariance of face representations in human ventral visual cortex: fMRI evidence. Neuroimage 26:1128-1139.

Einhäuser W, Stout J, Koch C, Carter O (2008) Pupil dilation reflects perceptual selection and predicts subsequent stability in perceptual rivalry. Proc Natl Acad Sci U S A 105:1704-1709. 
Engel SA, Furmanski CS (2001) Selective adaptation to color contrast in human primary visual cortex. J Neurosci 21:3949-3954.

Engel SA (2005) Adaptation of oriented and unoriented color-selective neurons in human visual areas. Neuron 45:613-623.

Epstein R, Graham KS, Downing PE (2003) Viewpoint-specific scene representations in human parahippocampal cortex. Neuron 37:865-876.

Epstein RA, Higgins JS, Thompson-Schill SL (2005) Learning places from views: variation in scene processing as a function of experience and navigational ability. J Cogn Neurosci 17:73-83.

Ewbank MP, Schluppeck D, Andrews TJ (2005) fMR-adaptation reveals a distributed representation of inanimate objects and places in human visual cortex. Neuroimage 28:268-279.

Fahrenfort JJ, Scholte HS, Lamme VA (2008) The spatiotemporal profile of cortical processing leading up to visual perception. J Vis 8:12.

Fang F, Murray SO, Kersten D, He S (2005) Orientation-tuned FMRI adaptation in human visual cortex.J Neurophysiol 94:4188-4195.

Fang F, Murray SO, He S (2007) Duration-Dependent fMRI Adaptation and Distributed Viewer-Centered Face Representation in Human Visual Cortex. Cereb Cortex 17:1402-1411.

Farrell JE, Shepard RN (1981) Shape, orientation, and apparent rotational motion. J Exp Psychol Hum Percept Perform 7:477-486.

Felleman DJ, Van Essen DC (1991) Distributed hierarchical processing in the primate cerebral cortex. Cereb Cortex 1:1-47.

Ferrera VP, Nealey TA, Maunsell JH (1994) Responses in macaque visual area V4 following inactivation of the parvocellular and magnocellular LGN pathways.J Neurosci 14:2080-2088.

ffytche DH, Guy CN, Zeki S (1995) The parallel visual motion inputs into areas V1 and V5 of human cerebral cortex. Brain 118:1375-1394.

ffytche DH, Zeki S (1996) Brain activity related to the perception of illusory contours. Neuroimage 3:104-108.

Field GD, Chichilnisky EJ (2007) Information processing in the primate retina: circuitry and coding. Annu Rev Neurosci 30:1-30.

Finlay D, von Grünau MW (1987) Some experiments on the breakdown effect in apparent motion. Percept Psychophys 42:526-534.

Fischl B, Sereno MI, Tootell RB, Dale AM (1999) High-resolution intersubject averaging and a coordinate system for the cortical surface. Hum Brain Mapp 8:272-284.

Foote SL, Morrison JH (1987) Extrathalamic modulation of cortical function. Annu Rev Neurosci 10:67-95.

Forman SD, Cohen JD, Fitzgerald M, Eddy WF, Mintun MA, Noll DC (1995) Improved assessment of significant activation in functional magnetic resonance imaging (fMRI): use of a cluster-size threshold. Magn Reson Med 33:636-647.

Foster DH, Gilson SJ (2002) Recognizing novel three-dimensional objects by summing signals from parts and views. Proc Biol Sci 269:1939-1947.

Frahm J, Bruhn H, Merboldt KD, Hanicke W (1992) Dynamic MR imaging of human brain oxygenation during rest and photic stimulation. J Magn Reson Imaging 2:501-505. 
Fujita K (2001) Perceptual completion in rhesus monkeys (Macaca mulatta) and pigeons (Columbia livia). Percept Psychophys 63:115-125.

Fujita K, Ushitani T (2005) Better living by not completing: a wonderful peculiarity of pigeon vision? Behav Processes 69:59-66.

Fulvio JM, Singh M (2006) Surface geometry influences the shape of illusory contours. Acta Psychol (Amst) 123:20-40.

Gallant JL, Shoup RE, Mazer JA (2000) A human extrastriate area functionally homologous to macaque V4. Neuron 27:227-235.

Ganel T, Gonzalez CL, Valyear KF, Culham JC, Goodale MA, Kohler S (2006) The relationship between $\mathrm{fMRI}$ adaptation and repetition priming. Neuroimage 32:1432-1440.

Gauthier I, Hayward WG, Tarr MJ, Anderson AW, Skudlarski P, Gore JC (2002) BOLD activity during mental rotation and viewpoint-dependent object recognition. Neuron 34:161-171.

Gegenfurtner KR (2003) Cortical mechanisms of colour vision. Nat Rev Neurosci 4:563572.

Genovese CR, Lazar NA, Nichols T (2002) Thresholding of statistical maps in functional neuroimaging using the false discovery rate. Neuroimage 15:870-878.

Gerlach C, Aaside CT, Humphreys GW, Gade A, Paulson OB, Law I (2002) Brain activity related to integrative processes in visual object recognition: bottom-up integration and the modulatory influence of stored knowledge. Neuropsychologia 40:12541267.

Gilbert CD, Wiesel TN (1992) Receptive field dynamics in adult primary visual cortex. Nature 356:150-152.

Goebel R, Khorram-Sefat D, Muckli L, Hacker H, Singer W (1998) The constructive nature of vision: direct evidence from functional magnetic resonance imaging studies of apparent motion and motion imagery. Eur J Neurosci 10:1563-1573.

Goebel R, Esposito F, Formisano E (2006) Analysis of Functional Image Analysis Contest (FIAC) data with BrainVoyager QX: From single-subject to cortically aligned group general linear model analysis and self-organizing group independent component analysis. Human Brain Mapping 27:392-401.

Gold JM, Murray RF, Bennett PJ, Sekuler AB (2000) Deriving behavioural receptive fields for visually completed contours. Curr Biol 10:663-666.

Goodale MA, Milner AD (1992) Separate visual pathways for perception and action. Trends Neurosci 15:20-25.

Grefkes C, Fink GR (2005) The functional organization of the intraparietal sulcus in humans and monkeys. J Anat 207:3-17.

Gregory RL (1966) Eye and Brain. London:Weidenfeld and Nicholson.

Grill-Spector K, Kushnir T, Hendler T, Edelman S, Itzchak Y, Malach R (1998) A sequence of object-processing stages revealed by $\mathrm{fMRI}$ in the human occipital lobe. Hum Brain Mapp 6:316-328.

Grill-Spector K, Kushnir T, Edelman S, Avidan G, Itzchak Y, Malach R (1999) Differential processing of objects under various viewing conditions in the human lateral occipital complex. Neuron 24:187-203.

Grill-Spector K, Malach R (2001) fMR-adaptation: a tool for studying the functional properties of human cortical neurons. Acta Psychol (Amst) 107:293-321. 
Grill-Spector K (2003) The neural basis of object perception. Curr Opin Neurobiol 13:159-166.

Grill-Spector K, Henson R, Martin A (2006) Repetition and the brain: neural models of stimulus-specific effects. Trends Cogn Sci 10:14-23.

Grosof DH, Shapley RM, Hawken MJ (1993) Macaque V1 neurons can signal 'illusory' contours. Nature 365:550-552.

Grossberg S, Rudd ME (1992) Cortical dynamics of visual motion perception: shortrange and long-range apparent motion. Psychol Rev 99:78-121.

Hagler DJ, Jr., Riecke L, Sereno MI (2007) Parietal and superior frontal visuospatial maps activated by pointing and saccades. Neuroimage 35:1562-1577.

Halgren E, Mendola J, Chong CD, Dale AM (2003) Cortical activation to illusory shapes as measured with magnetoencephalography. Neuroimage 18:1001-1009.

Haxby JV, Gobbini MI, Furey ML, Ishai A, Schouten JL, Pietrini P (2001) Distributed and overlapping representations of faces and objects in ventral temporal cortex. Science 293:2425-2430.

Haynes J-D, Rees G (2005a) Predicting the orientation of invisible stimuli from activity in human primary visual cortex. Nat Neurosci 8:686-691.

Haynes JD, Rees G (2005b) Predicting the stream of consciousness from activity in human visual cortex. Curr Biol 15:1301-1307.

Haynes JD, Rees G (2006) Decoding mental states from brain activity in humans. Nat Rev Neurosci 7:523-534.

Haynes JD, Sakai K, Rees G, Gilbert S, Frith C, Passingham RE (2007) Reading hidden intentions in the human brain. Curr Biol 17:323-328.

Hayward WG (2003) After the viewpoint debate: where next in object recognition? Trends Cogn Sci 7:425-427.

Heckman GM, Bouvier SE, Carr VA, Harley EM, Cardinal KS, Engel SA (2007) Nonlinearities in rapid event-related fMRI explained by stimulus scaling. Neuroimage 34:651-660.

Henson RN, Shallice T, Dolan R (2000) Neuroimaging evidence for dissociable forms of repetition priming. Science 287:1269-1272.

Henson RN, Shallice T, Gorno-Tempini ML, Dolan RJ (2002) Face repetition effects in implicit and explicit memory tests as measured by fMRI. Cereb Cortex 12:178-186.

Henson RN (2003) Neuroimaging studies of priming. Prog Neurobiol 70:53-81.

Henson RN, Rugg MD (2003) Neural response suppression, haemodynamic repetition effects, and behavioural priming. Neuropsychologia 41:263-270.

Henson RN, Rylands A, Ross E, Vuilleumeir P, Rugg MD (2004) The effect of repetition lag on electrophysiological and haemodynamic correlates of visual object priming. Neuroimage 21:1674-1689.

Henson RN, Mouchlianitis E (2007) Effect of spatial attention on stimulus-specific haemodynamic repetition effects. Neuroimage 35:1317-1329.

Hochbeerg J, McAlister E (1953) A quantitative approach to figural "goodness". J Exp Psychol 46:361.

Hoeth F (1968) Bevorzugte Richtungen bei stroboskopischen Alternativbewegungen. Psychol Beiträge 10:494-527.

Hotson JR, Braun D, Herzberg W, Boman D (1994) Transcranial magnetic stimulation of extrastriate cortex degrades human motion direction discrimination. Vision Res 34:2115-2123. 
Hubel DH, Wiesel TN (1968) Receptive fields and functional architecture of monkey striate cortex. J Physiol 195:215-243.

Huettel SA, McCarthy G (2000) Evidence for a refractory period in the hemodynamic response to visual stimuli as measured by MRI. Neuroimage 11:547-553.

Huettel SA, Obembe OO, Song AW, Woldorff MG (2004) The BOLD fMRI refractory effect is specific to stimulus attributes: evidence from a visual motion paradigm. Neuroimage 23:402-408.

Huk AC, Ress D, Heeger DJ (2001) Neuronal basis of the motion aftereffect reconsidered. Neuron 32:161-172.

Huxlin KR, Saunders RC, Marchionini D, Pham HA, Merigan WH (2000) Perceptual deficits after lesions of inferotemporal cortex in macaques. Cereb Cortex 10:671-683.

Ishai A, Pessoa L, Bikle PC, Ungerleider LG (2004) Repetition suppression of faces is modulated by emotion. Proc Natl Acad Sci U S A 101:9827-9832.

James TW, Humphrey GK, Gati JS, Menon RS, Goodale MA (1999) Repetition priming and the time course of object recognition: an fMRI study. Neuroreport 10:1019-1023.

James TW, Humphrey GK, Gati JS, Menon RS, Goodale MA (2000) The effects of visual object priming on brain activation before and after recognition. Curr Biol 10:10171024.

James TW, Humphrey GK, Gati JS, Menon RS, Goodale MA (2002) Differential effects of viewpoint on object-driven activation in dorsal and ventral streams. Neuron 35:793-801.

James TW, Gauthier I (2006) Repetition-induced changes in BOLD response reflect accumulation of neural activity. Hum Brain Mapp 27:37-46.

Jeannerod M, Arbib MA, Rizzolatti G, Sakata H (1995) Grasping objects: the cortical mechanisms of visuomotor transformation. Trends Neurosci 18:314-320.

Jiang X, Bradley E, Rini RA, Zeffiro T, Vanmeter J, Riesenhuber M (2007) Categorization training results in shape- and category-selective human neural plasticity. Neuron 53:891-903.

Jiang Y, Boehler CN, Nonnig N, Duzel E, Hopf JM, Heinze HJ, Schoenfeld MA (2008) Binding 3-d object perception in the human visual cortex. J Cogn Neurosci 20:553562.

Johnson SC, Baxter LC, Susskind-Wilder L, Connor DJ, Sabbagh MN, Caselli RJ (2004) Hippocampal adaptation to face repetition in healthy elderly and mild cognitive impairment. Neuropsychologia 42:980-989.

Johnson SP (2004) Development of perceptual completion in infancy. Psychol Sci 15:769-775.

Jolicoeur P (1990) Identification of misoriented objects: a dualsystems theory. Mind and Language 5:387-410.

Jurcoane A, Choubey B, Muckli L, Sireteanu R (2007) A pilot study for investigating cortical binocularity in humans using fMRI adaptation. Strabismus 15:33-37.

Kamitani Y, Tong F (2005) Decoding the visual and subjective contents of the human brain. Nat Neurosci 8:679-685.

Kamitani Y,Tong F (2006) Decoding seen and attended motion directions from activity in the human visual cortex. Curr Biol 16:1096-1102.

Kanizsa G (1976) Subjective contours. Sci Am 234:48-52. 
Kanizsa G, Renzi P, Conte S, Compostela C, Guerani L (1993) Amodal completion in mouse vision. Perception 22:713-721.

Kanwisher N, Chun MM, McDermott J, Ledden PJ (1996) Functional imaging of human visual recognition. Brain Res Cogn Brain Res 5:55-67.

Kawabata H, Gyoba J, Inoue H, Ohtsubo H (1999) Visual completion of partly occluded grating in infants under 1 month of age.Vision Res 39:3586-3591.

Kellman PJ, Shipley TF (1991) A theory of visual interpolation in object perception. Cognit Psychol 23:141-221.

Kellman PJ, Garrigan P, Shipley TF, Keane BP (2007) Interpolation processes in object perception: reply to Anderson (2007). Psychol Rev 114:488-508.

Kohler A, Haddad L, Singer W, Muckli L (2008) Deciding what to see: The role of intention and attention in the perception of apparent motion. Vision Res 48:1096-1106.

Kohn A, Movshon JA (2003) Neuronal adaptation to visual motion in area MT of the macaque. Neuron 39:681-691.

Kohn A, Movshon JA (2004) Adaptation changes the direction tuning of macaque MT neurons. Nat Neurosci 7:764-772.

Kohn A (2007) Visual adaptation: physiology, mechanisms, and functional benefits. J Neurophysiol 97:3155-3164.

Kolers PA (1964) The illusion of movement. Sci Am 211:98-106.

Komatsu $\mathrm{H}$ (2006) The neural mechanisms of perceptual filling-in. Nat Rev Neurosci 7:220-231.

Konen CS, Kastner S (2008) Two hierarchically organized neural systems for object information in human visual cortex. Nat Neurosci 11:224-231.

Korte A (1915) Kinematoskopische Untersuchungen. Z Psychol 72:194-296.

Kourtzi Z, Shiffrar M (1997) One-shot view invariance in a moving world. Psychological Science 8:461-466.

Kourtzi Z, Shiffrar M (1999) The visual representation of three-dimensional, rotating objects. Acta Psychol (Amst) 102:265-292.

Kourtzi Z, Kanwisher N (2000) Cortical regions involved in perceiving object shape. J Neurosci 20:3310-3318.

Kourtzi Z, Shiffrar M (2001) Visual representation of malleable and rigid objects that deform as they rotate. J Exp Psychol Hum Percept Perform 27:335-355.

Kourtzi Z, Kanwisher N (2001) Representation of perceived object shape by the human lateral occipital complex. Science 293:1506-1509.

Kourtzi Z, Erb M, Grodd W, Bülthoff HH (2003a) Representation of the perceived 3-D object shape in the human lateral occipital complex. Cereb Cortex 13:911-920.

Kourtzi Z, Tolias AS, Altmann CF, Augath M, Logothetis NK (2003b) Integration of local features into global shapes: monkey and human FMRI studies. Neuron 37:333-346.

Kourtzi Z, Huberle E (2005) Spatiotemporal characteristics of form analysis in the human visual cortex revealed by rapid event-related fMRI adaptation. Neuroimage 28:440-452.

Koutstaal W, Wagner AD, Rotte M, Maril A, Buckner RL, Schacter DL (2001) Perceptual specificity in visual object priming: functional magnetic resonance imaging evidence for a laterality difference in fusiform cortex. Neuropsychologia 39:184-199.

Kovacs G, Vogels R, Orban GA (1995) Selectivity of macaque inferior temporal neurons for partially occluded shapes. J Neurosci 15:1984-1997. 
Koyama S, Sasaki Y, Andersen GJ, Tootell RB, Matsuura M, Watanabe T (2005) Separate processing of different global-motion structures in visual cortex is revealed by FMRI. Curr Biol 15:2027-2032.

Krekelberg B, Vatakis A, Kourtzi Z (2005) Implied motion from form in the human visual cortex. J Neurophysiol 94:4373-4386.

Krekelberg B, Boynton GM, van Wezel RJ (2006) Adaptation: from single cells to BOLD signals. Trends Neurosci 29:250-256.

Kriegeskorte N, Sorger B, Naumer M, Schwarzbach J, van den Boogert E, Hussy W, Goebel R (2003) Human cortical object recognition from a visual motion flowfield. J Neurosci 23:1451-1463.

Kriegeskorte N, Goebel R, Bandettini P (2006) Information-based functional brain mapping. Proc Natl Acad Sci U S A 103:3863-3868.

Kriegeskorte N, Formisano E, Sorger B, Goebel R (2007) Individual faces elicit distinct response patterns in human anterior temporal cortex. Proc Natl Acad Sci U S A 104:20600-20605.

Kruggel F, Herrmann CS, Wiggins CJ, von Cramon DY (2001) Hemodynamic and electroencephalographic responses to illusory figures: recording of the evoked potentials during functional MRI. Neuroimage 14:1327-1336.

Lamme VA (2006) Towards a true neural stance on consciousness. Trends Cogn Sci 10:494-501.

Larsen A, Madsen KH, Lund TE, Bundesen C (2006) Images of illusory motion in primary visual cortex.J Cogn Neurosci 18:1174-1180.

Larsson J, Amunts K, Gulyas B, Malikovic A, Zilles K, Roland PE (1999) Neuronal correlates of real and illusory contour perception: functional anatomy with PET. Eur J Neurosci 11:4024-4036.

Larsson J, Heeger DJ (2006) Two retinotopic visual areas in human lateral occipital cortex. J Neurosci 26:13128-13142.

Larsson J, Landy MS, Heeger DJ (2006) Orientation-selective adaptation to first- and second-order patterns in human visual cortex. J Neurophysiol 95:862-881.

Lee $\mathrm{H}$, Vecera SP (2005) Visual cognition influences early vision: the role of visual shortterm memory in amodal completion. Psychol Sci 16:763-768.

Lee TS, Nguyen M (2001) Dynamics of subjective contour formation in the early visual cortex. Proc Natl Acad Sci U S A 98:1907-1911.

Lennie P (1998) Single units and visual cortical organization. Perception 27:889-935.

Lerner Y, Hendler T, Malach R (2002) Object-completion effects in the human lateral occipital complex. Cereb Cortex 12:163-177.

Lerner Y, Harel M, Malach R (2004) Rapid completion effects in human high-order visual areas. Neuroimage 21:516-526.

Leventhal AG, Wang Y, Schmolesky MT, Zhou Y (1998) Neural correlates of boundary perception. Vis Neurosci 15:1107-1118.

Levy I,Schluppeck D, Heeger DJ, Glimcher PW (2007) Specificity of human cortical areas for reaches and saccades. J Neurosci 27:4687-4696.

Li L, Miller EK, Desimone R (1993) The representation of stimulus familiarity in anterior inferior temporal cortex.J Neurophysiol 69:1918-1929.

Li W, Piech V, Gilbert CD (2008) Learning to link visual contours. Neuron 57:442-451. 
Liu LC, Plomp G, van Leeuwen C, loannides AA (2006) Neural correlates of priming on occluded figure interpretation in human fusiform cortex. Neuroscience 141:15851597.

Liu T, Slotnick SD, Yantis S (2004) Human MT+ mediates perceptual filling-in during apparent motion. Neuroimage 21:1772-1780.

Liu T, Larsson J, Carrasco M (2007) Feature-based attention modulates orientation-selective responses in human visual cortex. Neuron 55:313-323.

Logothetis NK, Pauls J, Bülthoff HH, Poggio T (1994) View-dependent object recognition by monkeys. Curr Biol 4:401-414.

Lustig C, Buckner RL (2004) Preserved neural correlates of priming in old age and dementia. Neuron 42:865-875.

Maccotta L, Buckner RL (2004) Evidence for neural effects of repetition that directly correlate with behavioral priming. J Cogn Neurosci 16:1625-1632.

Maertens M, Pollmann S (2005) fMRI reveals a common neural substrate of illusory and real contours in V1 after perceptual learning. J Cogn Neurosci 17:1553-1564.

Maertens M, Pollmann S (2007) Illusory contours do not pass through the "blind spot". J Cogn Neurosci 19:91-101.

Malach R, Reppas JB, Benson RR, Kwong KK, Jiang H, Kennedy WA, Ledden PJ, Brady TJ, Rosen BR, Tootell RB (1995) Object-related activity revealed by functional magnetic resonance imaging in human occipital cortex. Proc Natl Acad Sci U S A 92:81358139.

Malach R, Levy I, Hasson U (2002) The topography of high-order human object areas. Trends Cogn Sci 6:176-184.

Marr D, Nishihara HK (1978) Representation and recognition of the spatial organization of three-dimensional shapes. Proc R Soc Lond B Biol Sci 200:269-294.

Matsuyoshi D, Hirose N, Mima T, Fukuyama H, Osaka N (2007) Repetetive transcranial magnetic stimulation of human MT+ reduces apparent motion perception. Neurosci Lett 429:131-135.

Maunsell JH, Van Essen DC (1983) The connections of the middle temporal visual area (MT) and their relationship to a cortical hierarchy in the macaque monkey. J Neurosci 3:2563-2586.

McKyton A, Zohary E (2007) Beyond Retinotopic Mapping:The Spatial Representation of Objects in the Human Lateral Occipital Complex. Cereb Cortex 17:1164-1172.

McMahon DB, Olson CR (2007) Repetition suppression in monkey inferotemporal cortex: relation to behavioral priming. J Neurophysiol 97:3532-3543.

McNamara TP, Diwadkar VA, Blevins WA, Valiquette CM (2006) Representations of apparent rotation. Visual Cognition 13:273-307.

Meienbrock A, Naumer MJ, Doehrmann O, Singer W, Muckli L (2007) Retinotopic effects during spatial audio-visual integration. Neuropsychologia 45:531-539.

Mendola JD, Dale AM, Fischl B, Liu AK, Tootell RB (1999) The representation of illusory and real contours in human cortical visual areas revealed by functional magnetic resonance imaging. J Neurosci 19:8560-8572.

Meng M, Remus DA, Tong F (2005) Filling-in of visual phantoms in the human brain. Nat Neurosci 8:1248-1254. 
Merchant H, Battaglia-Mayer A, Georgopoulos AP (2005) Decoding of path-guided apparent motion from neural ensembles in posterior parietal cortex. Exp Brain Res 161:532-540.

Merigan WH, Maunsell JH (1993) How parallel are the primate visual pathways? Annu Rev Neurosci 16:369-402.

Michotte A, Thinès G, Crabbé G (1964) Les compléments amodaux des structures perceptives. Louvain: Publications Universitaires, Studia Psychologica.

Mikami A, Newsome WT, Wurtz RH (1986a) Motion selectivity in macaque visual cortex. I. Mechanisms of direction and speed selectivity in extrastriate area MT. J Neurophysiol 55:1308-1327.

Mikami A, Newsome WT, Wurtz RH (1986b) Motion selectivity in macaque visual cortex. II. Spatiotemporal range of directional interactions in MT and V1. J Neurophysiol 55:1328-1339.

Mikami A (1991) Direction selective neurons respond to short-range and long-range apparent motion stimuli in macaque visual area MT. Int J Neurosci 61:101-112.

Miller EK, Li L, Desimone R (1993) Activity of neurons in anterior inferior temporal cortex during a short-term memory task. J Neurosci 13:1460-1478.

Milner AD, Goodale MA (2008) Two visual systems re-viewed. Neuropsychologia 46:774785.

Mitsumatsu $\mathrm{H}$, Yokosawa K (2003) Efficient extrapolation of the view with a dynamic and predictive stimulus. Perception 32:969-983.

Montaser-Kouhsari L, Landy MS, Heeger DJ, Larsson J (2007) Orientation-selective adaptation to illusory contours in human visual cortex. J Neurosci 27:2186-2195.

Moore C, Engel SA (2001) Neural response to perception of volume in the lateral occipital complex. Neuron 29:277-286.

Morrison JH, Foote SL (1986) Noradrenergic and serotoninergic innervation of cortical, thalamic, and tectal visual structures in Old and New World monkeys. J Comp Neurol 243:117-138.

Muckli L, Kriegeskorte N, Lanfermann H, Zanella FE, Singer W, Goebel R (2002) Apparent motion: event-related functional magnetic resonance imaging of perceptual switches and states. J Neurosci 22:RC219.

Muckli L, Kohler A, Kriegeskorte N, Singer W (2005) Primary visual cortex activity along the apparent-motion trace reflects illusory perception. PLoS Biol 3:e265.

Muckli L, Kiess S, Tonhausen N, Singer W, Goebel R, Sireteanu R (2006) Cerebral correlates of impaired grating perception in individual, psychophysically assessed human amblyopes. Vision Res 46:506-526.

Müller JR, Metha AB, Krauskopf J, Lennie P (1999) Rapid adaptation in visual cortex to the structure of images. Science 285:1405-1408.

Munger MP, Solberg JL, Horrocks KK, Preston AS (1999) Representational momentum for rotations in depth: effects of shadings and axis. J Exp Psychol Learn Mem Cogn 25:157-171.

Munk MH, Linden DE, Muckli L, Lanfermann H, Zanella FE, Singer W, Goebel R (2002) Distributed cortical systems in visual short-term memory revealed by event-related functional magnetic resonance imaging. Cereb Cortex 12:866-876.

Murray MM, Wylie GR, Higgins BA, Javitt DC, Schroeder CE, Foxe JJ (2002) The spatiotemporal dynamics of illusory contour processing: combined high-density electri- 
cal mapping, source analysis, and functional magnetic resonance imaging. J Neurosci 22:5055-5073.

Murray MM, Foxe DM, Javitt DC, Foxe JJ (2004) Setting boundaries: brain dynamics of modal and amodal illusory shape completion in humans. J Neurosci 24:6898-6903.

Murray MM, Imber ML, Javitt DC, Foxe $\mathrm{JJ}$ (2006) Boundary completion is automatic and dissociable from shape discrimination. J Neurosci 26:12043-12054.

Murray SO, Olshausen BA, Woods DL (2003) Processing shape, motion and three-dimensional shape-from-motion in the human cortex. Cereb Cortex 13:508-516.

Murray SO, Wojciulik E (2004) Attention increases neural selectivity in the human lateral occipital complex. Nat Neurosci 7:70-74.

Murray SO, Olman CA, Kersten D (2006) Spatially specific FMRI repetition effects in human visual cortex. J Neurophysiol 95:2439-2445.

Naccache L, Dehaene S (2001) The priming method: imaging unconscious repetition priming reveals an abstract representation of number in the parietal lobes. Cereb Cortex 11:966-974.

Nakayama K, Shimojo S, Silverman GH (1989) Stereoscopic depth: its relation to image segmentation, grouping, and the recognition of occluded objects. Perception 18:55-68.

Neuhaus W (1930) Experimentelle Untersuchung der Scheinbewegung. Arch Gesamte Psychol 75:315-458.

Newsome WT, Britten KH, Movshon JA (1989) Neuronal correlates of a perceptual decision. Nature 341:52-54.

Newsome WT, Mikami A, Wurtz RH (1986) Motion selectivity in macaque visual cortex. III. Psychophysics and physiology of apparent motion. J Neurophysiol 55:13401351.

Newsome WT, Paré EB (1988) A selective impairment of motion perception following lesions of the middle temporal visual area (MT). J Neurosci 8:2201-2211.

$\mathrm{Ng} \mathrm{M}$, Ciaramitaro VM, Anstis S, Boynton GM, Fine I (2006) Selectivity for the configural cues that identify the gender, ethnicity, and identity of faces in human cortex. Proc Natl Acad Sci U S A 103:19552-19557.

Nishida S, Sasaki Y, Murakami I, Watanabe T, Tootell RB (2003) Neuroimaging of direction-selective mechanisms for second-order motion. J Neurophysiol 90:3242-3254.

Norman KA, Polyn SM, Detre GJ, Haxby JV (2006) Beyond mind-reading: multi-voxel pattern analysis of fMRI data. Trends Cogn Sci 10:424-430.

Ogawa S, Lee TM, Kay AR, Tank DW (1990) Brain magnetic resonance imaging with contrast dependent on blood oxygenation. Proc Natl Acad Sci U S A 87:9868-9872.

Op de Beeck HP, Haushofer J, Kanwisher NG (2008) Interpreting fMRI data:maps, modules and dimensions. Nat Rev Neurosci 9:123-135.

Orban GA, Sunaert S, Todd JT, Van Hecke P, Marchal G (1999) Human cortical regions involved in extracting depth from motion. Neuron 24:929-940.

Orban GA, Fize D, Peuskens H, Denys K, Nelissen K, Sunaert S, Todd J, Vanduffel W (2003) Similarities and differences in motion processing between the human and macaque brain: evidence from fMRI. Neuropsychologia 41:1757-1768.

Paradis AL, Cornilleau-Peres V, Droulez J, Van De Moortele PF, Lobel E, Berthoz A, Le Bihan D, Poline JB (2000) Visual perception of motion and 3-D structure from motion: an fMRI study. Cereb Cortex 10:772-783. 
Pasupathy A, Connor CE (2001) Shape representation in area V4: position-specific tuning for boundary conformation.J Neurophysiol 86:2505-2519.

Pasupathy A (2006) Neural basis of shape representation in the primate brain. Prog Brain Res 154:293-313.

Peterhans E, von der Heydt R (1989) Mechanisms of contour perception in monkey visual cortex. Il. Contours bridging gaps. J Neurosci 9:1749-1763.

Peterhans E, von der Heydt R (1993) Functional organization of area V2 in the alert macaque. Eur J Neurosci 5:509-524.

Peuskens H, Claeys KG, Todd JT, Norman JF, Van Hecke P, Orban GA (2004) Attention to 3-D shape, 3-D motion, and texture in 3-D structure from motion displays. J Cogn Neurosci 16:665-682.

Pillow J, Rubin N (2002) Perceptual completion across the vertical meridian and the role of early visual cortex. Neuron 33:805-813.

Plomp G, Liu L, van Leeuwen C, loannides AA (2006) The "mosaic stage" in amodal completion as characterized by magnetoencephalography responses. J Cogn Neurosci 18:1394-1405.

Polonsky A, Blake R, Braun J, Heeger DJ (2000) Neuronal activity in human primary visual cortex correlates with perception during binocular rivalry. Nat Neurosci 3:11531159.

Priebe NJ, Lisberger SG (2002) Constraints on the source of short-term motion adaptation in macaque area MT. II. tuning of neural circuit mechanisms. J Neurophysiol 88:370-382.

Priebe NJ, Churchland MM, Lisberger SG (2002) Constraints on the source of short-term motion adaptation in macaque area MT. I. the role of input and intrinsic mechanisms. J Neurophysiol 88:354-369.

Qiu FT, Von der Heydt R (2005) Figure and ground in the visual cortex: v2 combines stereoscopic cues with gestalt rules. Neuron 47:155-166.

Qiu FT, von der HR (2007) Neural representation of transparent overlay. Nat Neurosci 10:283-284.

Quiroga RQ, Mukamel R, Isham EA, Malach R, Fried I (2008) Human single-neuron responses at the threshold of conscious recognition. Proc Natl Acad Sci U S A 105:35993604.

Ramachandran VS, Anstis SM (1983) Extrapolation of motion path in human visual perception. Vision Res 23:83-85.

Ramsden BM, Hung CP, Roe AW (2001) Real and illusory contour processing in area V1 of the primate: a cortical balancing act. Cereb Cortex 11:648-665.

Rauschenberger R, Yantis S (2001) Masking unveils pre-amodal completion representation in visual search. Nature 410:369-372.

Rauschenberger R, Liu T, Slotnick SD, Yantis S (2006) Temporally unfolding neural representation of pictorial occlusion. Psychol Sci 17:358-364.

Reber PJ, Gitelman DR, Parrish TB, Mesulam MM (2005) Priming effects in the fusiform gyrus: changes in neural activity beyond the second presentation. Cereb Cortex 15:787-795.

Redies C, Crook JM, Creutzfeldt OD (1986) Neuronal responses to borders with and without luminance gradients in cat visual cortex and dorsal lateral geniculate nucleus. Exp Brain Res 61:469-481. 
Rees G, Kreiman G, Koch C (2002) Neural correlates of consciousness in humans. Nat Rev Neurosci 3:261-270.

Rensink RA, Enns JT (1998) Early completion of occluded objects. Vision Res 38:24892505.

Riesenhuber M, Poggio T (1999) Hierarchical models of object recognition in cortex. Nat Neurosci 2:1019-1025.

Ringo JL (1996) Stimulus specific adaptation in inferior temporal and medial temporal cortex of the monkey. Behav Brain Res 76:191-197.

Sack AT, Kohler A, Linden DE, Goebel R, Muckli L (2006) The temporal characteristics of motion processing in hMT/V5+: combining fMRI and neuronavigated TMS. Neurolmage 29:1326-1335.

Salzman CD, Britten KH, Newsome WT (1990) Cortical microstimulation influences perceptual judgements of motion direction. Nature 346:174-177.

Salzman CD, Murasugi CM, Britten KH, Newsome WT (1992) Microstimulation in visual area MT: effects on direction discrimination performance. J Neurosci 12:2331-2355.

Salzman CD, Newsome WT (1994) Neural mechanisms for forming a perceptual decision. Science 264:231-237.

Sasaki Y, Watanabe T (2004) The primary visual cortex fills in color. Proc Natl Acad Sci U S A 101:18251-18256.

Sawamura H, Georgieva S, Vogels R, Vanduffel W, Orban GA (2005) Using functional magnetic resonance imaging to assess adaptation and size invariance of shape processing by humans and monkeys. J Neurosci 25:4294-4306.

Sawamura H, Orban GA, Vogels R (2006) Selectivity of neuronal adaptation does not match response selectivity: a single-cell study of the FMRI adaptation paradigm. Neuron 49:307-318.

Sayres R, Grill-Spector K (2006) Object-selective cortex exhibits performance-independent repetition suppression. J Neurophysiol 95:995-1007.

Schacter DL, Buckner RL, Koutstaal W (1998) Memory, consciousness and neuroimaging. Philos Trans R Soc Lond B Biol Sci 353:1861-1878.

Schacter DL, Dobbins IG, Schnyer DM (2004) Specificity of priming: a cognitive neuroscience perspective. Nat Rev Neurosci 5:853-862.

Schiller Pv (1933) Stroboskopische Alternativversuche. Psychol Forsch 17:179-214.

Schacter DL, Wig GS, Stevens WD (2007) Reductions in cortical activity during priming. Curr Opin Neurobiol 17:171-176.

Schroeder CE, Mehta AD, Givre SJ (1998) A spatiotemporal profile of visual system activation revealed by current source density analysis in the awake macaque. Cereb Cortex 8:575-592.

Seghier M, Dojat M, Delon-Martin C, Rubin C, Warnking J, Segebarth C, Bullier J (2000) Moving illusory contours activate primary visual cortex: an fMRI study. Cereb Cortex 10:663-670.

Seghier ML, Vuilleumier P (2006) Functional neuroimaging findings on the human perception of illusory contours. Neurosci Biobehav Rev 30:595-612.

Sehatpour P, Molholm S, Javitt DC, Foxe JJ (2006) Spatiotemporal dynamics of human object recognition processing: an integrated high-density electrical mapping and functional imaging study of "closure" processes. Neuroimage 29:605-618. 
Sekuler AB, Palmer SE (1992) Perception of Partly Occluded Objects - A Microgenetic Analysis. Journal of Experimental Psychology-General 121:95-111.

Sekuler AB, Lee JA, Shettleworth SJ (1996) Pigeons do not complete partly occluded figures. Perception 25:1109-1120.

Serences JT, Boynton GM (2007) Feature-based attentional modulations in the absence of direct visual stimulation. Neuron 55:301-312.

Sereno MI, Pitzalis S, Martinez A (2001) Mapping of contralateral space in retinotopic coordinates by a parietal cortical area in humans. Science 294:1350-1354.

Shepard RN, Metzler J (1971) Mental rotation of three-dimensional objects. Science 171:701-703.

Shepard RN, Judd SA (1976) Perceptual illusion of rotation of three-dimensional objects. Science 191:952-954.

Sheth BR, Sharma J, Rao SC, Sur M (1996) Orientation maps of subjective contours in visual cortex. Science 274:2110-2115.

Shmuel A, Korman M, Sterkin A, Harel M, Ullman S, Malach R, Grinvald A (2005) Retinotopic axis specificity and selective clustering of feedback projections from V2 to V1 in the owl monkey. J Neurosci 25:2117-2131.

Silvanto J, Cowey A, Lavie N, Walsh V (2005) Striate cortex (V1) activity gates awareness of motion. Nat Neurosci 8:143-144.

Simons JS, Koutstaal W, Prince S, Wagner AD, Schacter DL (2003) Neural mechanisms of visual object priming: evidence for perceptual and semantic distinctions in fusiform cortex. Neuroimage 19:613-626.

Singh M (2004) Modal and amodal completion generate different shapes. Psychol Sci 15:454-459.

Smith AT, Greenlee MW, Singh KD, Kraemer FM, Hennig J (1998) The processing of firstand second-order motion in human visual cortex assessed by functional magnetic resonance imaging (fMRI). J Neurosci 18:3816-3830.

Smith AT, Singh KD, Williams AL, Greenlee MW (2001) Estimating receptive field size from $\mathrm{fMRI}$ data in human striate and extrastriate visual cortex. Cereb Cortex 11:1182-1190.

Sobotka S, Ringo JL (1994) Stimulus specific adaptation in excited but not in inhibited cells in inferotemporal cortex of macaque. Brain Res 646:95-99.

Soon CS, Venkatraman V, Chee MW (2003) Stimulus repetition and hemodynamic response refractoriness in event-related fMRI. Hum Brain Mapp 20:1-12.

Sovrano VA, Bisazza A (2008) Recognition of partly occluded objects by fish. Anim Cogn 11:161-166.

Spitzer H, Desimone R, Moran J (1988) Increased attention enhances both behavioral and neuronal performance. Science 240:338-340.

Stanley DA, Rubin N (2003) fMRI activation in response to illusory contours and salient regions in the human lateral occipital complex. Neuron 37:323-331.

Stepniewska I, Collins CE, Kaas JH (2005) Reappraisal of DL/V4 boundaries based on connectivity patterns of dorsolateral visual cortex in macaques. Cereb Cortex 15:809-822.

Stern CE, Corkin S, Gonzalez RG, Guimaraes AR, Baker JR, Jennings PJ, Carr CA, Sugiura RM, Vedantham V, Rosen BR (1996) The hippocampal formation participates in no- 
vel picture encoding: evidence from functional magnetic resonance imaging. Proc Natl Acad Sci U S A 93:8660-8665.

Sterzer P, Russ MO, Preibisch C, Kleinschmidt A (2002) Neural correlates of spontaneous direction reversals in ambiguous apparent visual motion. Neuroimage 15:908-916.

Sterzer P, Eger E, Kleinschmidt A (2003) Responses of extrastriate cortex to switching perception of ambiguous visual motion stimuli. Neuroreport 14:2337-2341.

Sterzer P, Haynes JD, Rees G (2006) Primary visual cortex activation on the path of apparent motion is mediated by feedback from hMT+/V5. Neuroimage 32:1308-1316.

Sterzer P, Kleinschmidt A (2005) A neural signature of colour and luminance correspondence in bistable apparent motion. Eur J Neurosci 21:3097-3106.

Sterzer P, Kleinschmidt A (2007) A neural basis for inference in perceptual ambiguity. Proc Natl Acad Sci USA 104:323-328.

Stone JV (1999) Object recognition: view-specificity and motion-specificity. Vision Research 39:4032-4044.

Sugita Y (1999) Grouping of image fragments in primary visual cortex. Nature 401:269272.

Sunaert S, Van Hecke P, Marchal G, Orban GA (1999) Motion-responsive regions of the human brain. Exp Brain Res 127:355-370.

Supèr $H$, Spekreijse $H$, Lamme VAF (2001) Two distinct modes of sensory processing observed in monkey primary visual cortex (V1). Nat Neurosci 4:304-310.

Swisher JD, Halko MA, Merabet LB, McMains SA, Somers DC (2007) Visual topography of human intraparietal sulcus. J Neurosci 27:5326-5337.

Talairach J,Tournoux P (1988) Co-planar stereotaxic atlas of the human brain, 3-dimensional proportional systems: an approach to cerebral imaging. New York: Thieme Medical Publishers.

Tanaka K (2003) Columns for complex visual object features in the inferotemporal cortex: clustering of cells with similar but slightly different stimulus selectivities. Cereb Cortex 13:90-99.

Tarr MJ, Pinker S (1989) Mental rotation and orientation-dependence in shape recognition. Cognit Psychol 21:233-282.

Tarr MJ, Bülthoff HH (1995) Is human object recognition better described by geon structural descriptions or by multiple views? Comment on Biederman and Gerhardstein (1993). J Exp Psychol Hum Percept Perform 21:1494-1505.

Thiel CM, Henson RN, Morris JS, Friston KJ, Dolan RJ (2001) Pharmacological modulation of behavioral and neuronal correlates of repetition priming. J Neurosci 21:68466852.

Thiel CM, Henson RN, Dolan RJ (2002) Scopolamine but not lorazepam modulates face repetition priming: a psychopharmacological fMRI study. Neuropsychopharmacology 27:282-292.

Thirion B, Pinel P, Meriaux S, Roche A, Dehaene S, Poline JB (2007) Analysis of a large $\mathrm{fMRI}$ cohort: Statistical and methodological issues for group analyses. Neuroimage 35:105-120.

Tolias AS, Smirnakis SM, Augath MA, Trinath T, Logothetis NK (2001) Motion processing in the macaque: revisited with functional magnetic resonance imaging. J Neurosci 21:8594-8601. 
Tolias AS, Keliris GA, Smirnakis SM, Logothetis NK (2005) Neurons in macaque area V4 acquire directional tuning after adaptation to motion stimuli. Nat Neurosci 8:591593.

Tong F, Engel SA (2001) Interocular rivalry revealed in the human cortical blind-spot representation. Nature 411:195-199.

Tong F (2003) Primary visual cortex and visual awareness. Nat Rev Neurosci 4:219-229. Tootell RB, Mendola JD, Hadjikhani NK, Ledden PJ, Liu AK, Reppas JB, Sereno MI, Dale AM (1997) Functional analysis of V3A and related areas in human visual cortex. J Neurosci 17:7060-7078.

Tootell RB, Hadjikhani NK, Vanduffel W, Liu AK, Mendola JD, Sereno MI, Dale AM (1998a) Functional analysis of primary visual cortex (V1) in humans. Proc Natl Acad Sci U S A 95:811-817.

Tootell RB, Hadjikhani N, Hall EK, Marrett S, Vanduffel W, Vaughan JT, Dale AM (1998b) The retinotopy of visual spatial attention. Neuron 21:1409-1422.

Tootell RBH, Reppas JB, Kwong KK, Malach R, Born RT, Brady TJ, Rosen BR, Belliveau JW (1995) Functional analysis of human MT and related visual cortical areas using magnetic resonance imaging. J Neurosci 15:3215-3230.

Treue S, Husain M, Andersen RA (1991) Human perception of structure from motion. Vision Res 31:59-75.

Tsao DY, Vanduffel W, Sasaki Y, Fize D, Knutsen TA, Mandeville JB, Wald LL, Dale AM, Rosen BR, Van E, Livingstone MS, Orban GA, Tootell RB (2003) Stereopsis activates V3A and caudal intraparietal areas in macaques and humans. Neuron 39:555-568.

Tse PU (1999) Volume completion. Cognit Psychol 39:37-68.

Tulving E, Schacter DL (1990) Priming and human memory systems. Science 247:301306.

Turk-Browne N,Yi DJ, Leber A, Chun M (2007) Visual quality determines the direction of neural repetition effects. Cereb Cortex 17:425-433.

Turk-Browne NB, Yi DJ, Chun MM (2006) Linking implicit and explicit memory:common encoding factors and shared representations. Neuron 49:917-927.

Ungerleider LG, Mishkin M (1982) Two cortical visual systems. In: Analysis of Visual Behaviour (Ingle DJ, Goodale MA, Mansfield RJW, eds), pp 549-586. Cambridge (MA): The MIT Press.

Ungerleider LG, Desimone R (1986) Cortical connections of visual area MT in the macaque. J Comp Neurol 248:190-222.

Ushitani T, Fujita K (2005) Pigeons do not perceptually complete partly occluded photos of food: an ecological approach to the "pigeon problem". Behav Processes 69:67-78.

Valyear KF, Culham JC, Sharif N, Westwood D, Goodale MA (2006) A double dissociation between sensitivity to changes in object identity and object orientation in the ventral and dorsal visual streams: a human fMRI study. Neuropsychologia 44:218-228.

Van Atteveldt N, Formisano E, Goebel R, Blomert L (2004) Integration of letters and speech sounds in the human brain. Neuron 43:271-282.

Van Essen DC, Maunsell JHR, Bixby JL (1981) The middle temporal visual area in the macaque: myeloarchitecture, connections, functional properties and topographic organization. J Comp Neurol 199:293-326. 
Van Turennout M, Ellmore T, Martin A (2000) Long-lasting cortical plasticity in the object naming system. Nat Neurosci 3:1329-1334.

Van Turennout M, Bielamowicz L, Martin A (2003) Modulation of neural activity during object naming: effects of time and practice. Cereb Cortex 13:381-391.

Vinberg J, Grill-Spector K (2008) Representation of shapes, edges, and surfaces across multiple cues in the human visual cortex.J Neurophysiol 99:1380-1393.

Von der Heydt R, Peterhans E, Baumgartner G (1984) Illusory contours and cortical neuron responses. Science 224:1260-1262.

Von der Heydt R, Peterhans E (1989) Mechanisms of contour perception in monkey visual cortex. I. Lines of pattern discontinuity. J Neurosci 9:1731-1748.

Vuilleumier P, Henson RN, Driver J, Dolan RJ (2002) Multiple levels of visual object constancy revealed by event-related fMRI of repetition priming. Nat Neurosci 5:491499.

Vuilleumier P, Schwartz S, Duhoux S, Dolan RJ, Driver J (2005) Selective attention modulates neural substrates of repetition priming and "implicit" visual memory: suppressions and enhancements revealed by FMRI.J Cogn Neurosci 17:1245-1260.

Vuong QC, Tarr MJ (2004) Rotation direction affects object recognition. Vision Res 44:1717-1730.

Wagemans J, van LR, Scholl BJ (2006) Introduction to Michotte's heritage in perception and cognition research. Acta Psychol (Amst) 123:1-19.

Wallach H, Oconnell DN (1953) The Kinetic Depth Effect. Journal of Experimental Psychology 45:205-217.

Wandell BA, Brewer AA, Dougherty RF (2005) Visual field map clusters in human cortex. Philos Trans R Soc Lond B Biol Sci 360:693-707.

Wandell BA, Dumoulin SO, Brewer AA (2007) Visual field maps in human cortex. Neuron 56:366-383.

Wässle H (2004) Parallel processing in the mammalian retina. Nat Rev Neurosci 5:747757.

Weigelt S, Kourtzi Z, Kohler A, Singer W, Muckli L (2007a) The cortical representation of objects rotating in depth. J Neurosci 27:3864-3874.

Weigelt S, Singer W, Muckli L (2007b) Separate cortical stages in amodal completion revealed by functional magnetic resonance adaptation. BMC Neurosci 8:70.

Weigelt S (2007) Seeing things: illusory contours in the human visual brain. J Neurosci 27:5269-5270.

Welchman AE, Deubelius A, Conrad V, Bülthoff HH, Kourtzi Z (2005) 3D shape perception from combined depth cues in human visual cortex. Nat Neurosci 8:820-827.

Wertheimer M (1912) Experimentelle Studien über das Sehen von Bewegung. Z Psychol 61:161-265.

Wig GS, Grafton ST, Demos KE, Kelley WM (2005) Reductions in neural activity underlie behavioral components of repetition priming. Nat Neurosci 8:1228-1233.

Williams MA, Berberovic N, Mattingley JB (2007) Abnormal FMRI adaptation to unfamiliar faces in a case of developmental prosopamnesia. Curr Biol 17:1259-1264.

Williams ZM, Elfar JC, Eskandar EN, Toth LJ, Assad JA (2003) Parietal activity and the perceived direction of ambiguous apparent motion. Nat Neurosci 6:616-623.

Wouterlood D, Boselie F (1992) A good-continuation model of some occlusion phenomena. Psychol Res 54:267-277. 
Xu Y, Chun MM (2006) Dissociable neural mechanisms supporting visual short-term memory for objects. Nature 440:91-95.

Xu Y, Turk-Browne NB, Chun MM (2007) Dissociating task performance from fMRI repetition attenuation in ventral visual cortex. J Neurosci 27:5981-5985.

Yang CY, Hsieh JC, Chang Y (2006) An MEG study into the visual perception of apparent motion in depth. Neurosci Lett 403:40-45.

Yantis S, Nakama T (1998) Visual interactions in the path of apparent motion. Nat Neurosci 1:508-512.

Yi DJ, Chun MM (2005) Attentional modulation of learning-related repetition attenuation effects in human parahippocampal cortex.J Neurosci 25:3593-3600.

Yi DJ, Kelley TA, Marois R, Chun MM (2006) Attentional modulation of repetition attenuation is anatomically dissociable for scenes and faces. Brain Res 1080:53-62.

Yoshino A, Kawamoto M, Yoshida T, Kobayashi N, Shigemura J, Takahashi Y, Nomura S (2006) Activation time course of responses to illusory contours and salient region:a high-density electrical mapping comparison. Brain Res 1071:137-144.

Zago L, Fenske MJ, Aminoff E, Bar M (2005) The rise and fall of priming: how visual exposure shapes cortical representations of objects. Cereb Cortex 15:1655-1665.

Zeki S, Watson JDG, Lueck CJ, Friston KJ, Kennard C, Frackowiak RSJ (1991) A direct demonstration of functional specialization in human visual cortex. J Neurosci 11:641649.

Zeki S, Watson JD, Frackowiak RS (1993) Going beyond the information given: the relation of illusory visual motion to brain activity. Proc Biol Sci 252:215-222.

Zhaoping $L$ (2005) Border ownership from intracortical interactions in visual area v2. Neuron 47:143-153.

Zhou H, Friedman HS, Von der Heydt R (2000) Coding of border ownership in monkey visual cortex. J Neurosci 20:6594-6611.

Zhuo Y, Zhou TG, Rao HY, Wang JJ, Meng M, Chen M, Zhou C, Chen L (2003) Contributions of the visual ventral pathway to long-range apparent motion. Science 299:417420.

Zihl J, von Cramon D, Mai N (1983) Selective disturbance of movement vision after bilateral brain damage. Brain 106:313-340. 


\section{Abbreviations}

\begin{tabular}{|c|c|}
\hline $2 \mathrm{D}, 3 \mathrm{D}$ & two dimensional, three dimensional \\
\hline $\begin{array}{l}\text { ACl } \\
\text { ant }\end{array}$ & $\begin{array}{l}\text { attention-control task } \\
\text { anterior }\end{array}$ \\
\hline BOLD & blood-oxygenation level-dependent \\
\hline CS & central sulcus \\
\hline DMT & direction-of-motion task \\
\hline FDR & false discovery rate \\
\hline FFA & fusiform face area \\
\hline fMRI & functional magnetic resonance imaging \\
\hline GLM & general linear model \\
\hline hMT+ & human motion complex, also hMT/V5+ \\
\hline inf & inferior \\
\hline IOG & inferior occipital gyrus \\
\hline IPS & inferior parietal sulcus \\
\hline ISI & interstimulus interval \\
\hline IT, ITC & inferior temporal, inferior temporal cortex \\
\hline ITI & intertrial interval \\
\hline LO-1, LO-2 & lateral occipital-1 and -2 \\
\hline LOC & lateral occipital complex \\
\hline LOS & lateral occipital sulucs \\
\hline MRI & magnetic resonance imaging \\
\hline MT & mediotemporal, motion-sensitive region in monkeys \\
\hline MVPA & multi-voxel pattern analysis \\
\hline OIT & object-identification task \\
\hline pFG & posterior fusiform gyrus \\
\hline PMC & premotor cortex \\
\hline POS & parietooccipital sulcus \\
\hline post & posterior \\
\hline PPA & parahippocampal place area \\
\hline ROI & region of interest \\
\hline SEM & standard error of the mean \\
\hline SFM & structure from motion \\
\hline SMA & supplemental motor area \\
\hline SMC & sensorimotor cortex \\
\hline SOA & stimulus onset asynchrony \\
\hline SPC & superior parietal cortex \\
\hline SPFC & superior prefrontal cortex \\
\hline STS & superior temporal sulcus \\
\hline sup & superior \\
\hline TOS & transverse occipital sulcus \\
\hline TPJ & temporal parietal junction \\
\hline V1, V2, V3 etc. & visual areas 1, 2, 3 etc. \\
\hline VLPFC & ventral lateral prefrontal cortex \\
\hline VO-1 & ventral occipital-1 \\
\hline
\end{tabular}





\section{Acknowledgements}

Albert Einstein said: "If we knew what we were doing, it wouldn't be called research, would it?" Here I want to thank all the people who were there for me, when I didn't know what I was doing. 
My thanks go

to Lars Muckli for igniting the fMRI fire in me and the freedom he gave me to pursue my own research ideas,

to Axel Kohler for taking over the supervision after Lars went for cold and rainy weather, for being a patient teacher and research partner,

to Wolf Singer for his constant support, invaluable criticism, and the neurophysiological foundation of my work,

to Rainer Goebel for being my PhD supervisor at the University of Maastricht and even more for creating such an ingenious 'click and voyage' game,

to the members of my PhD committee, Jochen Kaiser, Chantal Kemner, Francesco di Salle, Petra Stoerig, Peter de Weerd, for the time and effort they spent reviewing my $\mathrm{PhD}$ thesis,

to all members of the functional neuroimaging group, past and present, who made and still make my time at the MPIH such a joy: Arjen Alink, Matthias Dhum, Erhan Genç, Amra Hodzic, Sean Lee, Yingjie Li, Katharina Pohl, Miriam Schwalm, Caspar Schwiedrzik, and Michael Wibral,

to all colleagues from the Department of Neurophysiology, past and present, who were and still are responsible for the pleasant and inspiring working atmosphere, especially Boris Ebisch, Sascha Gotthardt, Bruss Lima, Matthias Munk, Anne Schmidt and Christine Tillmann, to my friends and colleagues at the Institute of Medical Psychology, namely Christian Altmann, Christoph Bledowski, Linda Heinemann, Jochen Kaiser, Marcus Naumer, Benjamin Rahm, Jasper van den Bosch and Yavor Yalachkov,

to the members of the departments of administration, engineering and IT at MPIH for their administrative and technical assistance,

to all colleagues from the Brain Imaging Center Frankfurt for their support,

to all my subjects for their patience,

to Petra Janson for making me an Adobe Creative Suite addict,

to Dr. Yusuf from the department of neurosecurity for his special warm-hearted sense of humor that made me often leave the institute with a smile on my face, to Barbara Legrum for her commitment to the MinervaFemmeNet, to Ina Bornkessel-Schlesewsky for being my mentor,

to Paule Biaudet for her encouragement,

to Thomas Metzinger and the MIND group members for inspiring discussions,

to Amanda Kaas, my dearly missed ex-roommate, for being a wonderful listener and also for providing the Dutch summary of my thesis,

to Daniela Bertarelli and Julia Völz, my fast friends, for accompanying me through the years,

to Karen, Leif and Joscha Zentgraf and Axel Kohler (again) whose ears I've bent and shoulders l've leaned on often, and who cheered me on nevertheless,

an meine Großeltern Josefine und Karl-Heinz Weigelt und Magdalene Willmes für Ihre beständige und liebevolle Unterstützung,

to my parents Angelika and Bernd Weigelt and my brother Christian, for their love and encouragement,

to my husband Oliver Doehrmann for ... (words don't suffice). 


\section{Curriculum Vitae}


Sarah Weigelt was born on November 31978 in Solingen, Germany. In 1998 she completed her high school education at the Landrat Lucas Gymnasium Leverkusen. She took up her studies of philosophy (1998) and psychology (1999) at the Heinrich Heine University, Düsseldorf. Her interest in the mind and the brain led her to focus on neurophilosophy and experimental biopsychology. After research assistant positions with Prof. Dr. Clemens Kirschbaum, Prof. Dr. Petra Stoerig, and Prof. Dr. Rüdiger Seitz she moved to Frankfurt am Main to accomplish her M.Sc. thesis on "Interactions of motion and form perception in the human visual brain" at the Max Planck Institute for Brain Research. In march 2004 she was awarded her M.Sc. in psychology and in october 2004 her M.A. in philosophy by Heinrich Heine University, Düsseldorf. Before returning to the Max Planck Institute for Brain Research in order to pursue her Ph.D. work, a childhood dream sent her off to Louisiana, USA, to study the cognitive capabilities of chimpanzees in the lab of Prof. Dr. Daniel Povinelli. Luckily, she soon started missing the sound of an MRI machine and since then has settled on research involving huge magnetic devices. In April 2005 she started her Ph.D. research at the Max Planck Institute for Brain Research (Department of Neurophysiology headed by Prof. Dr. Wolf Singer) under the supervision of Dr. Lars Muckli and Dr. Axel Kohler. Currently she is working at the Max Planck Institute for Brain Research as a postdoctoral researcher.

\section{Publications}

\section{peer-reviewed}

Weigelt, S., Muckli, L., and Kohler, A. (2008). The method of functional magnetic resonance adaptation in visual neuroscience. Reviews in the Neurosciences, in press.

Weigelt, S., Kourtzi, Z., Kohler, A., Singer, W., and Muckli, L. (2007). The cortical representation of objects rotating in depth. Journal of Neuroscience, 27:3864-3874.

Weigelt, S., Singer, W., and Muckli, L. (2007). Separate cortical stages in amodal completion revealed by functional magnetic resonance adaptation. BMC Neuroscience, 8: 70.

Weigelt, S. (2007). Seeing things: illusory contours in the human visual brain. Journal of Neuroscience, 27:5269-5270.

\section{manuscripts}

Kaas, A., Weigelt, S., Roebroeck, A., Kohler, A., and Muckli, L. (submitted). Imagery of a moving object: the role of occipital cortex and human MT/V5+

Maus, G.W., Weigelt, S., Nijhawan, R., and Muckli, L. (submitted). Neural correlates of motion extrapolation in early visual cortex: An fMRI study

\section{book}

Weigelt, S. Interactions of motion and form processing. A neuroimaging investigation. VDM Verlag Dr. Müller, Saarbrücken: 2007 (published MSc thesis).

\section{abstracts}

Weigelt, S., Singer, W., and Kohler, A. (2008). Interactions of position- and direction-dependent fMRI adaptation. Poster at the 38th Annual Meeting of the SfN, Washington, USA. 
Doehrmann, O., Altmann, C.F., Weigelt, S., Kaiser, J., and Naumer, M.J. (2008). Multisensory integration investigated by $\mathrm{fMRI}$ adaptation. Poster by Doehrmann at the 38th Annual Meeting of the SfN, Washington, USA.

Kohler, A., Weigelt, S., Muckli, L., Singer, W., and Kriegeskorte, N. (2008). Patterns of fMRI activity representing perceived direction in apparent motion. Poster by Kohler at the 38th Annual Meeting of the SfN, Washington, USA.

Maus, G., Weigelt, S., Nijhawan, R., and Muckli, L. (2008). Activity in area V3A predicts positions of moving objects. Poster by Maus at the ECVP 2008, Utrecht, The Netherlands

Kaas, A., Weigelt, S., Roebroeck, A., Kohler, A., Singer, W., and Muckli, L. (2008). Imagery of a moving object affects activation patterns and directed influences of hMT/V5+, posterior parietal and early visual regions. Poster by Kaas at the 14th Annual Meeting of the OHBM, Melbourne, Australia.

Weigelt, S., Singer, W., and Kohler, A. (2008). FMRI adaptation in higher visual areas: inherited or intrinsic? Poster at the Tagung Psychologie \& Gehirn 2008, Magdeburg, Germany.

Kohler, A., Weigelt, S., Muckli, L., Singer, W., and Kriegeskorte, N. (2008) Patterns of fMRI activity representing perceived direction in long-range apparent motion; Poster at the Tagung Psychologie \& Gehirn 2008, Magdeburg, Germany.

Doehrmann, O., Altmann, C.F., Weigelt, S., Kaiser, J., and Naumer, M. (2008). Audio-visual repetition suppression and enhancement in occipital and temporal cortices as revealed by fMRI-adaptation. Poster at the Tagung Psychologie \& Gehirn 2008, Magdeburg, Germany.

Weigelt, S., Singer, W. and Muckli, L. (2007). Seeing and perceiving - visual completion in the human brain; Poster at the 37th Annual Meeting of the SfN, San Diego, USA.

Weigelt, S., Singer, W. and Muckli, L. (2007). Separate cortical stages in amodal completion revealed by functional magnetic resonance adaptation; Poster at the Tagung Psychologie \& Gehirn, Dortmund, Germany.

Kaas, A., Weigelt, S., Formisano, E., Linden, D., Singer, W., and Muckli, L. (2007). Imagined Motion: investigating effective connectivity between early visual areas, hMT/V5+ and parietal cortex; Poster by Kaas at the 13th Annual Meeting of the OHBM, Chicago, USA.

Weigelt, S., Singer, W. and Muckli, L. (2006). The perception of occluded shapes uncovered - a functional magnetic resonance adaptation study; Poster at the 12th Annual Meeting of the OHBM, Florence, Italy.

Weigelt, S., Singer, W. and Muckli, L. (2006).Verdeckte Teile von Objekten werden bereits in frühen visuellen Arealen ergänzt; Talk at the 48th Tagung experimentell arbeitender Psychologen, Mainz, Germany.

Weigelt, S., Kourtzi, Z., Kohler, A., Singer, W., and Muckli, L. (2004). Apparent Rotation: functional magnetic resonance adaptation of the illusory rotation path; Talk by Muckli at the 34th Annual Meeting of the SfN, San Diego, USA.

Muckli, L., Weigelt, S., Kohler, A., Singer, W., and Kourtzi, Z. (2004). Apparent Rotation: fMRI-adaptation of the illusory rotation path; Poster at the 10th Annual Meeting of the OHBM, Budapest, Hungary.

Weigelt, S., Kourtzi, Z., Singer, W., and Muckli, L. (2004). Interactions of Motion and Form Processing: an fMR-A study of Apparent Rotation. Talk held at the 46th Tagung experimentell arbeitender Psychologen, Giessen, Germany. 